\title{
Iterability for (transfinite) stacks
}

\author{
Farmer Schlutzenberg*† \\ WWU Münster
}

January 12, 2021

\begin{abstract}
We establish natural criteria under which normally iterable premice are iterable for stacks of normal trees. Let $\Omega$ be a regular uncountable cardinal. Let $m<\omega$ and $M$ be an $m$-sound premouse and $\Sigma$ be an $(m, \Omega+1)$-iteration strategy for $M$ (roughly, a normal $(\Omega+1)$-strategy).

We define a natural condensation property for iteration strategies, inflation condensation. We show that if $\Sigma$ has inflation condensation then $M$ is $(m, \Omega, \Omega+1)^{*}$-iterable (roughly, $M$ is iterable for length $\leq \Omega$ stacks of normal trees each of length $<\Omega$ ), and moreover, we define a specific such strategy $\Sigma^{\text {st }}$ and a reduction of stacks via $\Sigma^{\text {st }}$ to normal trees via $\Sigma$. If $\Sigma$ has the Dodd-Jensen property and $\operatorname{card}(M)<\Omega$ then $\Sigma$ has inflation condensation.

We also apply some of the techniques developed to prove that if $\Sigma$ has strong hull condensation (introduced independently by Steel), and $G$ is $V$-generic for an $\Omega$-cc forcing, then $\Sigma$ extends to an $(m, \Omega+1)$-strategy $\Sigma^{+}$for $M$ with strong hull condensation, in the sense of $V[G]$. Moreover, this extension is unique. We deduce that if $G$ is $V$-generic for a ccc forcing then $V$ and $V[G]$ have the same $\omega$-sound, $(\omega, \Omega+1)$-iterable premice which project to $\omega$.
\end{abstract}

\section{Introduction}

Let $M$ be a normally iterable premouse. Does it follow that $M$ is iterable for non-normal trees? We prove here the following partial positive result in this direction, which applies to both Mitchell-Steel indexed and $\lambda$-indexed premice. The notion inflation condensation is a certain condensation property for iteration strategies, defined in Definition 4.37. Roughly, it says that if there is a normal iteration strategy $\Sigma$ for $M$ with inflation condensation, then there is an iteration strategy $\Sigma^{*}$ for $M$ for normal stacks of iteration trees; that is, sequences of normal trees, appropriately formed:

${ }^{*}$ Editing partially funded by the Deutsche Forschungsgemeinschaft (DFG, German Research Foundation) under Germany's Excellence Strategy EXC 2044-390685587, Mathematics Münster: Dynamics-Geometry-Structure.

${ }^{\dagger}$ farmer.schlutzenberg@gmail.com,https://sites.google.com/site/schlutzenberg 
Theorem (9.1, 9.3). Let $M$ be an $m$-sound premouse, let $\Omega$ be a regular uncountable cardinal, let $\xi \in\{\Omega, \Omega+1\}$, let $\Sigma$ be an $(m, \xi)$-iteration strategy for $M$ and suppose that $\Sigma$ has inflation condensation. Then:

- if $\xi=\Omega$ then $M$ is $(m,<\omega, \Omega)^{*}$-iterable, and

- if $\xi=\Omega+1$ then $M$ is $(m, \Omega, \Omega+1)^{*}$-iterable.

Moreover, there is an iteration strategy $\Sigma^{*}$ witnessing this with $\Sigma \subseteq \Sigma^{*}$.

The background theory for the above theorem is ZF (actually, it is much more local than this). Likewise for the other results of the paper, except where indicated otherwise.

Recall here that an $(m, \alpha, \beta)^{*}$-iteration strategy is a winning strategy for player II in the iteration game $\mathcal{G}(M, m, \alpha, \beta)^{*}$; this is the variant, introduced in [23], of the iteration game $\mathcal{G}(M, m, \alpha, \beta)$ of [24, §4]. In both of these games, the players build a sequence $\left\langle\mathcal{T}_{\gamma}\right\rangle$ of length at most $\alpha$, consisting of normal iteration trees $\mathcal{T}_{\gamma}$, with $\mathcal{T}_{0}$ on $M, \mathcal{T}_{1}$ on the last model $M_{\infty}^{\mathcal{T}_{0}}$ of $\mathcal{T}_{0}$, etc. But in $\mathcal{G}(M, m, \alpha, \beta)^{*}$, if in some round $\gamma<\alpha$, a bona fide tree $\mathcal{T}_{\gamma}$ of length $\beta$ is reached, then player II automatically wins the entire game. Player I may of course end the round earlier, with some tree of successor length $<\beta .{ }^{1}$ The rules are spelled out explicitly in $\S 1.1$. The two games are only distinct when $\beta$ is a successor. For limit $\alpha$, an $(m,<\alpha, \beta)^{*}$-iteration strategy is likewise, except that if the game lasts through $\alpha$ rounds, with neither player having yet lost, then player II wins.

We will define explicitly a specific such strategy $\Sigma^{*}$ from $\Sigma$, denoted $\Sigma^{\text {st }}$. Trees via $\Sigma^{\text {st }}$ of length $<\Omega$ will lift to trees via $\Sigma$ of length $<\Omega$. Further, if $\Omega=\omega_{1}$ and $M$ is countable and we code $\Sigma \uparrow \mathrm{HC}$ and $\Sigma^{\text {st }} \uparrow \mathrm{HC}$ naturally with functions $\Sigma_{0}, \Sigma_{0}^{\text {st }}$ on the reals, then $\Sigma_{0}^{\text {st }}$ is $\Delta_{1}\left(\Sigma_{0}\right)$. (We do not know if one can improve on this complexity.)

The construction of $\Sigma^{\text {st }}$ breaks into two main pieces (we assume for the purposes of this discussion that all trees have length $<\Omega$ and player I does not make any artificial drops in model or degree at the beginning of rounds; that is, $\left(M^{\prime}, m^{\prime}\right)$ is the model and degree produced at the end of one round, then the next round is $m^{\prime}$-maximal on $M^{\prime}$ ). First, given a normal tree $\mathcal{T}$ via $\Sigma$ of successor length, we define a normal strategy $\Upsilon_{\mathcal{T}}^{\Sigma}$ for $M_{\infty}^{\mathcal{T}}$, together with a process which converts normal trees $\mathcal{U}$ on $M_{\infty}^{\mathcal{T}}$ via $\Upsilon_{\mathcal{T}}^{\Sigma}$ to normal trees $\mathcal{X}=\mathcal{W}_{\mathcal{T}}^{\Sigma}(\mathcal{U})$ on $M$ via $\Sigma$, and produces an embedding

$$
\varrho: M_{\infty}^{\mathcal{U}} \rightarrow M_{\infty}^{\mathcal{X}}
$$

when $\mathcal{U}$ has successor length. We then also have the normal strategy $\Upsilon_{\mathcal{X}}^{\Sigma}$ for $M_{\infty}^{\mathcal{X}}$. But using $\varrho$ we can copy trees on $M_{\infty}^{\mathcal{U}}$ to trees on $M_{\infty}^{\mathcal{X}}$. We can define a

\footnotetext{
${ }^{1}$ Whereas in $\mathcal{G}_{m}(M, \alpha, \beta)$, if $\beta$ is a successor ordinal and not all rounds have been played, then the game would continue, with the next round building a tree on $M_{\infty}^{\mathcal{T}}$. Actually, the author has always understood $\mathcal{G}(M, m, \alpha, \beta)$ and $(m, \alpha, \beta)$-iterability to be defined as we have just defined $\mathcal{G}(M, m, \alpha, \beta)^{*}$ and $(m, \alpha, \beta)^{*}$-iterability, due to misreading the definition in [24] at some point. The author thanks Gabriel Fernandes for pointing out this confusion over the definition. The author is not aware of any use of $(m, \alpha, \beta)$-iterability beyond $(m, \alpha, \beta)^{*}$ iterability in the literature.
} 
normal strategy $\Upsilon_{\mathcal{T}, \mathcal{U}}^{\Sigma}$, as the $\varrho$-pullback of $\Upsilon_{\mathcal{X}}^{\Sigma}$. So $M_{\infty}^{\mathcal{U}}$ is iterable, etc. So this first step leads immediately to a strategy for stacks of length $<\omega$. Second, given a limit $\eta$ and a stack $\overrightarrow{\mathcal{T}}$ of length $\eta$ in which each normal component is built using the process above (or a generalization thereof, if $\eta>\omega$ ) and corresponding sequence $\overrightarrow{\mathcal{X}}$ of normal trees, we show that there is a natural limit $\mathcal{X}$ of $\overrightarrow{\mathcal{X}}$, and that everything fits together in a sufficiently commutative fashion that the direct limit $M_{\infty}^{\overrightarrow{\mathcal{T}}}$ of the stack $\overrightarrow{\mathcal{T}}$ embeds into $M_{\infty}^{\mathcal{X}}$, so we can continue through longer stacks.

This overall process we call here normal realization, as the tree $\mathcal{X}$ is normal, but for example in the situation above, we need not have $M_{\infty}^{\mathcal{U}}=M_{\infty}^{\mathcal{X}}$. It is often called normalization elsewhere, and embedding normalization in [21], but we prefer to reserve the term normalization for a tighter process that we do not discuss here (that is, full normalization in the terminology of [21], where one gets a normal tree $\mathcal{X}$ for which $\left.M_{\infty}^{\mathcal{U}}=M_{\infty}^{\mathcal{X}}\right) .^{2}$

Using normal realization, we will also prove a theorem concerning the following iteration game:

1.1 Definition. Let $M$ be an $m$-sound premouse. In $\mathcal{G}_{\text {fin }}(M, m, \Omega+1)$, player I plays a finite length putative $m$-maximal stack $\overrightarrow{\mathcal{T}}=\left\langle\mathcal{T}_{i}\right\rangle_{i<k}$ of finite length trees $\mathcal{T}_{i}$, player I wins if $M_{\infty}^{\overrightarrow{\mathcal{T}}}$ is illfounded, and otherwise, the players proceed to play out the $(n, \Omega+1)$-iteration game on $M_{\infty}^{\overrightarrow{\mathcal{T}}}$ where $n=\operatorname{deg}^{\overrightarrow{\mathcal{T}}}(\infty)$.

(See $\S 1.1$ for explanations of terminology.) Note that unlike the main theorems on normal realization mentioned above, the following one requires no condensation hypothesis for $\Sigma .^{3}$

Theorem (9.6). Let $\Omega>\omega$ be regular and $M$ be $m$-sound $(m, \Omega+1)$-iterable. Then (i) player II has a winning strategy for $\mathcal{G}_{\text {fin }}(M, m, \Omega+1)$. Moreover, (ii) let $\overrightarrow{\mathcal{T}}=\left\langle\mathcal{T}_{i}\right\rangle_{i<\omega}$ be an $m$-maximal stack on $M$ consisting of finite length trees $\mathcal{T}_{i}$ (and note $\operatorname{lh}(\overrightarrow{\mathcal{T}})=\omega$ ). Then for all sufficiently large $i<\omega, b^{\mathcal{T}_{i}}$ does not drop in model or degree, player I makes no artificial drop in round $i$, and $M_{\infty}^{\overrightarrow{\mathcal{T}}}$ is wellfounded.

From the results in the paper we obtain the following equivalence of various forms of iterability, for countable premice. Strong hull condensation is another condensation property for iteration strategies, isolated by Steel; see $4.42 .{ }^{4}$ Inflation condensation and strong hull condensation have the same basic idea behind them; indeed inflation condensation just demands that certain instances of strong hull condensation hold, so the latter implies the former. The author

\footnotetext{
${ }^{2}$ Also, [21] deals with fine structural strategy mice, as well as pure $L[\mathbb{E}]$ mice, whereas here we consider pure $L[\mathbb{E}]$ mice, and also certain coarse structures, though many of the results adapt routinely.

${ }^{3}$ Part (i) of the theorem was used by the author in the presentation Fine structure from normal iterability at the 2015 Münster conference, and part (ii) provides a simplification of another fact used there.

${ }^{4}$ Strong hull condensation was defined by Steel, and inflation condensation by the author, independently of one another, at around the same time. Jensen also independently defined a similar condensation notion at around this time.
} 
does not know whether they are equivalent. The implication from (weak) DoddJensen to strong hull condensation, that is, Theorem 4.47, was pointed out to the author by Steel in $2017 .{ }^{5}$

1.2 Theorem. Assume DC. Let $\Omega>\omega$ be regular and $M$ be a countable $m$-sound premouse. Then the following are equivalent:

(a) $M$ is $(m, \Omega, \Omega+1)^{*}$-iterable.

(b) There is an $(m, \Omega+1)$-strategy for $M$ with inflation condensation.

(c) There is an $(m, \Omega+1)$-strategy for $M$ with strong hull condensation.

(d) There is an $(m, \Omega+1)$-strategy for $M$ with weak Dodd-Jensen.

(e) There is an $(m, \Omega, \Omega+1)^{*}$-strategy for $M$ with weak Dodd-Jensen.

Proof. (a) $\Rightarrow$ (e) is by [5] (only this implication uses DC), (e) $\Rightarrow$ (d) is trivial, (d) $\Rightarrow$ (c) by 4.47, (c) $\Rightarrow$ (b) by 4.44 , and (b) $\Rightarrow$ (a) by 9.1 .

We do not know whether DC is necessary above. But in Corollary 10.17 we do present a construction of an iteration strategy with weak Dodd-Jensen in a specific choiceless context.

Part of the methods in the paper also yield the following result, relating to extending a normal iteration strategy to a generic extension $V[G]$. While the construction of $\Sigma^{\text {st }}$ only demands inflation condensation of $\Sigma$, its proof uses strong hull condensation:

Theorem (7.3). Let $\Omega>\omega$ be regular. Let $M$ be an $m$-sound premouse. Let $\Sigma$ be an $(m, \Omega+1)$-strategy for $M$ with strong hull condensation. Let $\mathbb{P}$ be an $\Omega$-cc forcing and $G$ be $V$-generic for $\mathbb{P}$. Then in $V[G]$, there is a unique $(m, \Omega+1)$-strategy $\Sigma^{\prime}$ for $M$ such that $\Sigma \subseteq \Sigma^{\prime}$ and $\Sigma^{\prime}$ has inflation condensation. Moreover, $\Sigma^{\prime}$ has strong hull condensation.

As elsewhere, the background theory for the theorem above is ZF; the definition of $\Omega-c c$ in this general context is given in Definition 7.1.

Using the preceding results we deduce the following absoluteness facts:

Corollary $(7.6,10.12)$. Let $\Omega>\omega$ be regular. Let $M$ be a countable $m$-sound premouse and let e be an enumeration of $M$ in ordertype $\omega$. Let $\mathbb{P}$ be an $\Omega$-cc forcing and $G$ be $V$-generic for $\mathbb{P}$. Then:

- $V \models$ "There is an $(m, \Omega+1)$-strategy for $M$ with weak Dodd-Jensen with respect to $e "$ iff $V[G]$ satisfies the same statement.

- If $\Sigma$ is an (hence the unique) $(m, \Omega+1)$-strategy for $M$ with weak DoddJensen with respect to $e$, and $\Sigma^{\prime}$ likewise in $V[G]$, then $\Sigma \subseteq \Sigma^{\prime}$.

- Suppose that $V$ and $V[G]$ satisfy DC. Then $V \models$ " $M$ is $(m, \Omega, \Omega+1)^{*}$ iterable" iff $V[G]$ satisfies the same statement.

\footnotetext{
${ }^{5}$ That is, for $\lambda$-indexed premice; for MS-indexed premice there are additional technical considerations to deal with, as discussed here.
} 
We expect that given appropriate condensation properties for $\Sigma$, one should be able to deduce nice condensation properties for $\Sigma^{\text {st }}$. We prove one such result here. Plus-strong hull condensation, defined in 10.5, is a slight technical strengthening of strong hull condensation, and normal pullback consistency, defined in 10.1, is just pullback consistency for the normal strategy given by pullback under iteration maps which do not drop in model or degree.

Theorem (10.7). Let $\Omega>\omega$ be regular, and let $\Sigma$ be an $(m, \Omega+1)$-iteration strategy with plus-strong hull condensation. Then $\Sigma^{\text {st }}$ is normally pullback consistent.

1.3 Question. Our results suggest the following questions:

- If $\Omega>\omega$ is regular, does $(n, \Omega+1)$-iterability imply $(n, \Omega, \Omega+1)^{*}$ iterability, at least for countable premice?

- If $\Omega>\omega_{1}$ is regular and $M$ is uncountable and $(n, \Omega, \Omega+1)^{*}$-iterable, then does $M$ have an $\left(n, \omega_{1}+1\right)$-strategy with inflation condensation?

Why consider the methods in this paper? The author's initial motivation for working on these ideas was toward proving self-iterability facts in mice (particularly, non-tame mice). This involves an extension of the methods of [10] (that paper only applies to tame mice), using inflation condensation and arguments related to those in the proof of Theorem 7.3. We do not focus on this method in detail in this paper, but it is sketched in Remark 5.7. The second motivation was basically in wanting to understand the connection between normal iterability and iterability for stacks, and their role in the proofs of the standard fine structural properties of mice (such as solidity, etc). The first part of the author's work on this appeared in [18], and a full proof of the fine structural properties from normal iterability can be see in the preprint [14]. That proof relies heavily on Theorem 9.6. Looking forward, the key role of direct limit systems of mice in the analysis of the HOD of determinacy models and in the theory of Varsovian models of mice, also means that normalization can give new information about those direct limit models $M_{\infty}$. In fact, the unpublished work of Steel and the author on full normalization for infinite stacks (which adapts results of this paper and of [21]) gives that such models $M_{\infty}$ are in fact typically a normal iterate of some base mouse $M$ (not just embedded into a normal iterate). This could be useful in understanding those models. The methods are also useful generally in the Varsovian model analysis to appear in [7]. Finally, Steel's work [21] on comparison of iteration strategies (see below) provides a clear motivation for understanding the techniques.

Other people (including Mitchell, Steel, Neeman, Sargsyan, Fuchs, Schindler, Jensen and Siskind) have worked on aspects of normal realization; for further discussion see the introduction to [21]. Around the same time the author started this work, John Steel was working on related calculations, as a component of [21]. Steel presented his work on normal realization (which he calls normalization) for finite stacks of infinite trees, at the 3rd Münster conference on inner model 
theory, in July 2015, which the author attended. Part ${ }^{6}$ of the work in this paper was done by the author prior to being aware of Steel's work, and the remainder afterward. ${ }^{7}$ Our approach is also different, most importantly in that we have different axiomatic starting points, and different goals. In this paper we start with a normal iteration strategy with inflation condensation, and construct an iteration strategy for stacks from this. Steel starts more or less with an iteration strategy for stacks, demanding certain properties from this strategy, and uses these toward his strategy comparison. The notation and terminology we use is different from Steel's (as we have not attempted to align it with his); this also reflects a difference in how we approach the details of normal realization. However, many of the basic calculations and observations for dealing with finite stacks are the same. Around the same time we developed the methods for infinite stacks, Steel also worked out representative cases for a somewhat different ${ }^{8}$ approach to this problem. Some time after this, the authors discussed the two approaches together. But [21] does not deal with infinite stacks.

Also from around this time, Ronald Jensen also developed normal realization of finite stacks in the context of $\Sigma^{*}$-fine structure. The author sent him a draft version of the present paper containing the main arguments at the end of 2017, and Jensen then adapted the work contained here to infinite stacks in the $\Sigma^{*}$ context. His work is available in handwritten form in [3], and in the forthcoming $[2]$.

The author would like to thank Cody Dance and Jared Holshouser for a conversation on the topic, in roughly December 2014, during which the author first started to consider it seriously, and also John Steel for several conversations on the topic since July 2015.

The paper proceeds as follows. In $\S 1.1$ below we give a summary of basic terminology and notation. The results of the paper hold for iteration strategies for Mitchell-Steel (MS-)indexed and $\lambda$-indexed premice, and many of the results hold for a fairly broad class of coarse structures, weak coarse premice (wcpms). However, iteration trees on MS-indexed premice (formed by the standard rules) are somewhat inconvenient for the main arguments. So in $\S 2$ we discuss a reorganization of such iteration trees, which allows us to treat MS-indexed and $\lambda$-indexed premice in a simpler and uniform manner (except that then for various

\footnotetext{
${ }^{6}$ The work done prior to the Münster conference comprises basically of inflation $\mathcal{T} \rightsquigarrow \mathcal{X}$ for arbitrary normal trees $\mathcal{T}$, the notion of inflation condensation, genericity inflation for MSindexing, and normal realization of stacks of the form $(\mathcal{T}, \mathcal{U})$ where $\mathcal{T}$ is normal of finite length and $\mathcal{U}$ is normal of arbitrary length.

${ }^{7}$ Having earlier failed to understand infinite stacks, but motivated by Steel's suggestion during the Münster conference that one should be able to extend normal realization to them, the main ideas for that extension were determined by the author during the conference, and details finalized shortly thereafter. So by that time, the key material from $\S \S 4,6,8,9$ had been developed, and also $\S 5$ excluding genericity iteration for $\lambda$-indexing. The main ideas for the proof of Theorem 7.3 were found by the author in mid 2016, and some details corrected in August 2018. Theorem 10.7 also came in August 2018.

${ }^{8}$ In $\S 9.1 .2$, for limit $\eta$, the branches of the tree $\mathcal{Y}_{\eta}$ are determined directly by the given normal iteration strategy. Steel's approach appeared to the author to be somewhat more constructive, with branches of $\mathcal{Y}_{\eta}$ being determined instead by the trees $\mathcal{Y}_{\alpha}$ for $\alpha<\eta$ and maps between these. However, the author has not gone through the details.
} 
results we also need to give a short argument translating between these two forms of iteration trees and corresponding strategies). The reader who only wants to think about $\lambda$-indexed premice can safely skip this section. In $\S 3$ we define wcpms and iteration strategies for them.

The main content of the paper begins in $\S 4$. Here we introduce the key notions of the paper: tree embedding and inflation, leading to inflation condensation and strong hull condensation. A tree embedding $\Pi: \mathcal{T} \hookrightarrow \mathcal{X}$ embeds the structure of $\mathcal{T}$ (tree order, models and extenders) into the structure of $\mathcal{X}$ in a certain manner, but with a key difference to the hulls of trees in the sense of $[6$, $\S 1.6]$ : Each node $\alpha<\operatorname{lh}(\mathcal{T})$ is associated to a closed $\mathcal{X}$-interval $\left[\gamma_{\alpha}, \delta_{\alpha}\right]_{\mathcal{X}}$, and $M_{\alpha}^{\mathcal{T}}$ is embedded into $M_{\gamma_{\alpha}}^{\mathcal{X}}$, and $E_{\alpha}^{\mathcal{T}}$ is embedded into $E_{\delta_{\alpha}}^{\mathcal{X}}$, but maybe $\gamma_{\alpha}<\delta_{\alpha}$. An inflation of $\mathcal{T}$ is an iteration tree $\mathcal{X}$ in which each extender $E$ used in $\mathcal{X}$ is considered as either copied from $\mathcal{T}$ or as $\mathcal{T}$-inflationary. While building an inflation $\mathcal{X}$ we keep track of various tree embeddings from initial segments of $\mathcal{T}$ into $\mathcal{X}$. If $E_{\delta}^{\mathcal{X}}$ is copied from $\mathcal{T}$, then $\delta=\delta_{\alpha}$ for one of these tree embeddings $\mathcal{T} \uparrow(\alpha+2) \hookrightarrow \mathcal{X}$. The tree embeddings are "stretched" by the $\mathcal{T}$-inflationary extenders used in $\mathcal{X}$. We also introduce a lot of notation which will be needed throughout.

In $\S 5$ we describe techniques analogous to comparison of mice and genericity iteration of mice, but with mice replaced by iteration trees via a strategy with inflation condensation; these are called minimal simultaneous inflation and genericity inflation respectively. The comparison technique is key to our main results. We don't actually use the genericity inflation technique in the paper, but it is natural and seems useful. We also describe in Theorem 5.8 how genericity iteration for $\lambda$-indexed mice works in general.

In $\S 6$ we study the commutativity which results when we have three iteration trees $\mathcal{X}_{0}, \mathcal{X}_{1}$ and $\mathcal{X}_{2}$, and $\mathcal{X}_{i+1}$ is an inflation of $\mathcal{X}_{i}$ for $i=0,1$ (and given that $\mathcal{X}_{1}$ is an $\mathcal{X}_{0}$-terminal inflation of $\mathcal{X}_{0}$ ). We show that in this situation, $\mathcal{X}_{2}$ is an inflation of $\mathcal{X}_{0}$, and "everything commutes" in a natural sense. This result is essential in our analysis of infinite stacks of iteration trees in the construction of $\Sigma^{\text {st }}$; there we will deal with infinite sequences $\left\langle\mathcal{X}_{\alpha}\right\rangle_{\alpha<\eta}$ in which $\mathcal{X}_{\beta}$ is an inflation of $\mathcal{X}_{\alpha}$ for each $\alpha<\beta<\eta$.

In $\S 7$ we prove Theorem 7.3 , on extending iteration strategies with strong hull condensation to generic extensions.

Let $\mathcal{X}$ be an inflation of $\mathcal{T}$. With the definitions above, one's focus tends to be on the extenders of $\mathcal{X}$ which are copied from $\mathcal{T}$ as the central objects, while the $\mathcal{T}$-inflationary extenders are in the background. In $\S 8$ we give a second viewpoint which reverses this. Enumerating the $\mathcal{T}$-inflationary extenders as $\left\langle E_{\zeta^{\alpha}}^{\mathcal{X}}\right\rangle_{\alpha+1<i}$, we define a natural factor tree $<^{\mathcal{X} / \mathcal{T}}$, which is an iteration tree order on $\iota$. These things arise in the construction of $\Sigma^{\text {st }}$. Here when forming a tree $\mathcal{U}$ on $M_{\infty}^{\mathcal{T}}$, and the associated normal tree $\mathcal{X}=\mathcal{W}_{\mathcal{T}}^{\Sigma}(\mathcal{U})$, then $\mathcal{X}$ will be an inflation of $\mathcal{T}$, and we will have $<^{\mathcal{U}}=<^{\mathcal{X} / \mathcal{T}}$, and $E_{\alpha}^{\mathcal{U}}$ will embed into $E_{\zeta^{\alpha}}^{\mathcal{X}}$. We also introduce more bookkeeping which will be needed in the construction of $\Sigma^{\text {st }}$.

In $\S 9$ we give the construction of the stacks strategy $\Sigma^{\text {st }}$ and related proofs. 
Finally in $\S 10$ we establish some extra properties of $\Sigma^{\text {st }}$, given certain extra properties hold of $\Sigma$. The main result here is Theorem 10.7, on normal pullback consistency. We also use our results to give a construction of an iteration strategy with weak Dodd-Jensen in a certain choiceless context.

\subsection{Terminology}

See the end for an index of definitions. We give a summary here of the basic terminology and notation we use.

\subsubsection{General}

$\lfloor M\rfloor$ denotes the universe of structure $M$.

\subsubsection{Extenders and ultrapowers}

Given an extender $E$ over $M$, Ult $(M, E)$ denotes the ultrapower, formed from functions in $M, i_{E}^{M}: M \rightarrow \operatorname{Ult}(M, E)$ denotes the ultrapower embedding, and if $M$ is an $n$-sound premouse and $E$ is short, weakly amenable and $\operatorname{cr}(E)<\rho_{n}^{M}$, then $i_{E}^{M, n}: M \rightarrow \operatorname{Ult}_{n}(M, E)$ denotes the degree $n$ ultrapower embedding. We may write $i_{E}$ if $M$ is not emphasized. We write $\operatorname{cr}(E)$ for the critical point of $E, \lambda(E)=\lambda_{E}$ for $i_{E}(\operatorname{cr}(E)), \operatorname{lh}(E)$ for the length of $E$ or support of $E$ (we take all extenders to be a subset of $\mathcal{P}\left([\theta]^{<\omega}\right) \times[\lambda]^{<\omega}$ for some ordinals $\theta, \lambda$, and $\operatorname{lh}(E)$ is the least such $\lambda)$, and $\nu(E)=\nu_{E}$ for the strict sup of generators of $E$, and when $E$ is used in an iteration tree $\mathcal{T}, \widetilde{\nu}(E)$ denotes the exchange ordinal associated to $E$; this is explained further below. ${ }^{9}$ If $E$ is an extender over $V$, also write $\varrho(E)$ for the strength of $E$ (the largest $\alpha$ such that $V_{\alpha} \subseteq \operatorname{Ult}(V, E)$ ), Say an extender $E$ over $V$ is suitable iff $E$ is short and $\operatorname{lh}(E)=\nu(E)=\varrho(E)$. So a suitable extender is coded by a subset of $2^{\operatorname{cr}(E)}+\varrho(E)$.

\subsubsection{Premice}

The term wcpm (weak coarse premouse) is defined in $\S 3$.

The unqualified term premouse means either as in [25], or as in [24], except that we allow extenders of superstrong type to appear on the extender sequence (see [20] (2.43 and 2.44 of preprint v2 on arxiv.org) regarding this); here given a premouse $N$ with active extender $F$, we say that $F$ is of superstrong type if $\lambda(F)$ is the largest cardinal of $N$. The premice of [25] we call $\lambda$-indexed, and those of [24] MS-indexed. So if $M$ has $\lambda$-indexing then every extender in the extender sequence of $M$ is of superstrong type. The ISC (initial segment condition) is then as in [25] or [24] respectively.

Let $M, N$ be premice, or other similar structures. We write $M \unlhd N$ iff $M$ is an initial segment of $N$, and $M \triangleleft N$ iff $M \unlhd N$ and $M \neq N$. We write $F^{M}$ for the active extender of $M, \mathbb{E}^{M}$ denotes the extender sequence of $M$, excluding $F^{M}, \mathbb{E}_{+}^{M}$ denotes $\mathbb{E}^{M \uparrow} F^{M}, M^{\mathrm{pv}}$ denotes the passivization of $M$ (that is, if

\footnotetext{
${ }^{9}$ The notation should probably literally be $\widetilde{\nu}^{\mathcal{T}}(E)$, but $\mathcal{T}$ will be known from context.
} 
$M=\left(\mathcal{J}_{\alpha}^{\mathbb{E}}, \mathbb{E}, F\right)$ then $\left.M^{\mathrm{pv}}=\left(\mathcal{J}_{\alpha}^{\mathbb{E}}, \mathbb{E}, \emptyset\right)\right)$, and given a limit ordinal $\alpha \leq \mathrm{OR}^{N}$, $N \mid \alpha$ denotes the $M \unlhd N$ such that $\mathrm{OR}^{M}=\alpha$, and $N|| \alpha$ denotes $(N \mid \alpha)^{\mathrm{pv}}$.

If $M$ is a type 3 MS-indexed premouse, then $M^{\text {sq }}$ denotes the squash of $M$. If $N$ is a structure in the language of squashed premice, then $N^{\text {unsq }}$ denotes the unique such $M$ such that $M^{\mathrm{sq}}=N$, if this exists. For other kinds of MS-indexed premice $P, P^{\mathrm{sq}}=P^{\mathrm{unsq}}=P$. (But we do not define squashing in the context of u-fine structure $(\S 2))$.

Given premice $M, N$ and $m, n \leq \omega$ such that $M$ is $m$-sound and $N$ is $n$ sound, we write $(M, m) \unlhd(N, n)$ iff either $M \triangleleft N$ or $[M=N$ and $m \leq n]$. We write $(M, m) \triangleleft(N, n)$ iff $(M, m) \unlhd(N, n)$ and $(M, m) \neq(N, n)$. We similarly define $(M, m) \unlhd(N, n)$ and $(M, m) \triangleleft(N, n)$ when $M$ is $\mathrm{u}-m$-sound and $N$ is $\mathrm{u}$ - $n$-sound (see $\S 2$ ).

A segmented-premouse (seg-pm) is a structure $N$ satisfying all requirements of premice (either MS-indexed or $\lambda$-indexed), except that if $F^{N} \neq \emptyset$ then we do not require that $N$ satisfy the ISC (either in the sense of [24] or [25], as is appropriate); we still require in this case that $N$ has a largest cardinal $\delta$ and

$$
\operatorname{Ult}\left(N, F^{N}\right) \mid\left(\delta^{+}\right)^{\operatorname{Ult}\left(N, F^{N}\right)}=N \| \mathrm{OR}^{N},
$$

and all proper segments of seg-pms must satisfy the ISC. In particular, if $N$ is a premouse then $N$ is a seg-pm, and if $N$ is a seg-pm then $N^{\mathrm{pv}}$ is a premouse. If $N$ is active then ind $\left(F^{N}\right)$ (for index) denotes $\mathrm{OR}^{N}$. We also use "ind" for an analogous role in connection with coarse structures; see $\S 3$. Given a seg-pm $M$ with largest cardinal $\delta, \operatorname{lgcd}(M)$ denotes $\delta$. We extend the terminology and notation for premice mentioned above to seg-pms in the natural way.

\subsubsection{Fine structure}

We officially follow Mitchell-Steel fine structure, as simplified in [13]; however, the paper is predominantly not particularly dependent on which version of fine structure one uses. For "u-" fine structure, see $\S 2$. For the notation $\mathrm{Hull}_{m}^{M}$ and $\mathrm{cHull}_{m}^{M}$ see $[18, \S 1.1 .3]$.

\subsubsection{Iteration trees}

Beyond what is described here, there is also terminology specific to iteration trees introduced in $\S 4.1$. We formally understand iteration trees on premice and seg-pms basically as defined in [4], and in particular, of the form

$$
\mathcal{T}=\left(<^{\mathcal{T}}, \mathscr{D}^{\mathcal{T}}, \operatorname{deg}^{\mathcal{T}},\left\langle M_{\alpha}^{\mathcal{T}}\right\rangle_{\alpha<\lambda},\left\langle M_{\alpha+1}^{* \mathcal{T}}, E_{\alpha}^{\mathcal{T}}\right\rangle_{\alpha+1<\lambda}\right)
$$

where:

$-\operatorname{lh}(\mathcal{T})=\lambda \in[1, \mathrm{OR})$,

$-<^{\mathcal{T}}$ is the associated tree order on $\lambda$,

- $\mathscr{D}^{\mathcal{T}}$ is the set of all $\alpha+1<\lambda$ such that $\mathcal{T}$ drops at $\alpha+1$, 
$-\operatorname{deg}^{\mathcal{T}}: \lambda \rightarrow \omega+1$ is a $\operatorname{total}^{10}$ function,

- $M_{\alpha+1}^{* \mathcal{T}}$ is the model to which $E_{\alpha}^{\mathcal{T}}$ applies in forming $M_{\alpha+1}^{\mathcal{T}}$.

We take iteration trees on other structures with analogous formal structure. We also use the following notation (some of which is only relevant to trees on seg-pms):

- If $\alpha \leq^{\mathcal{T}} \beta$ then $(\alpha, \beta]_{\mathcal{T}}$ denotes the half-open $<^{\mathcal{T}}$-interval, and likewise for other such intervals.

$-\operatorname{pred}^{\mathcal{T}}(\alpha+1)$ denotes the $<^{\mathcal{T}}$-predecessor of $\alpha+1$ (so $\left.M_{\alpha+1}^{* \mathcal{T}} \unlhd M_{\text {pred }^{\mathcal{T}}(\alpha+1)}^{\mathcal{T}}\right)$,

- If $\alpha<^{\mathcal{T}} \beta$ then $\operatorname{succ}^{\mathcal{T}}(\alpha, \beta)$ denotes $\min \left((\alpha, \beta]_{\mathcal{T}}\right)$.

- If $\mathcal{T}$ has successor length $\alpha+1$, then $M_{\infty}^{\mathcal{T}}$ denotes $M_{\alpha}^{\mathcal{T}}$, and $\infty$ also denotes $\alpha$ in other related notation, and $b^{\mathcal{T}}$ denotes $[0, \infty]_{\mathcal{T}}$, the last branch of $\mathcal{T}$.

- If $(\alpha, \beta]_{\mathcal{T}} \cap \mathscr{D}^{\mathcal{T}}=\emptyset$ then $i_{\alpha \beta}^{\mathcal{T}}=i_{\alpha, \beta}^{\mathcal{T}}: M_{\alpha}^{\mathcal{T}} \rightarrow M_{\beta}^{\mathcal{T}}$ is the iteration map.

$-i_{\alpha+1}^{* \mathcal{T}}: M_{\alpha+1}^{* \mathcal{T}} \rightarrow M_{\alpha+1}^{\mathcal{T}}$ denotes the ultrapower map.

$-i_{\alpha+1, \beta}^{* \mathcal{T}}$ denotes $i_{\alpha+1, \beta}^{\mathcal{T}} \circ i_{\alpha+1}^{* \mathcal{T}}$, when this exists.

- $\mathscr{D}_{\mathrm{deg}}^{\mathcal{T}}$ denotes the set of all $\alpha+1<\lambda$ such that $\mathcal{T}$ drops in either model or degree at $\alpha+1$.

$-\operatorname{lh}(\mathcal{T})^{-}$denotes the set of all $\beta$ such that $\beta+1<\operatorname{lh}(\mathcal{T})$.

$-\widetilde{\nu}_{\alpha}^{\mathcal{T}}=\widetilde{\nu}\left(E_{\alpha}^{\mathcal{T}}\right)$ is the exchange ordinal associated to $E_{\alpha}^{\mathcal{T}}$; see below.

$-\operatorname{ex}_{\alpha}^{\mathcal{T}} \operatorname{denotes} M_{\alpha}^{\mathcal{T}} \mid \operatorname{ind}\left(E_{\alpha}^{\mathcal{T}}\right)$.

- if $\mathcal{T}$ has limit length, then if $\mathcal{T}$ is $k$-maximal (see below) on a seg-pm, then $\delta(\mathcal{T})$ denotes $\sup _{\alpha<\operatorname{lh}(\mathcal{T})} \operatorname{ind}\left(E_{\alpha}^{\mathcal{T}}\right)$, and if $\mathcal{T}$ is normal on a wcpm, then $\delta(\mathcal{T})$ denotes $\sup _{\alpha<\operatorname{lh}(\mathcal{T})} \varrho^{M_{\alpha}^{\mathcal{T}}}\left(E_{\alpha}^{\mathcal{T}}\right)$.

However, if $M_{0}^{\mathcal{T}}$ has MS-indexing then we can have $\operatorname{ind}\left(E_{\alpha}^{\mathcal{T}}\right)=\operatorname{ind}\left(E_{\alpha+1}^{\mathcal{T}}\right)$, because we allow superstrong extenders in $\mathbb{E}_{+}\left(M_{0}^{\mathcal{T}}\right)$. Considering this, an iteration tree $\mathcal{T}$ is $k$-maximal iff $\operatorname{deg}^{\mathcal{T}}(0)=k$ and $\mathcal{T}$ satisfies the requirements specified in [24, §3.1] for $k$-maximality, except that as in [20] and [18], we only require $\operatorname{ind}\left(E_{\alpha}^{\mathcal{T}}\right) \leq \operatorname{ind}\left(E_{\beta}^{\mathcal{T}}\right)$ when $\alpha+1<\beta+1<\operatorname{lh}(\mathcal{T})$, not $\operatorname{ind}\left(E_{\alpha}^{\mathcal{T}}\right)<\operatorname{ind}\left(E_{\beta}^{\mathcal{T}}\right) .{ }^{11}$ So if an iteration tree is both $k$-maximal and $j$-maximal, then $k=j .{ }^{12}$ This helps

\footnotetext{
${ }^{10}$ The requirement of totality might differ from [4], depending on the reader's interpretation.

${ }^{11}$ See [20] (2.43 and 2.44 in preprint v2 on arxiv.org) and [18, §1.1.6] for further discussion. The algorithm for comparison (by least disagreement) should also be slightly adjusted as in one of those papers (see Footnote 33; though in the proof of Corollary 7.6 we use the conventional algorithm). In a draft of this article on arxiv.org, $k$-maximal was defined inadvertently as in [24] (even though Remark 2.44 of [20] was also mentioned), which does not suffice.

${ }^{12}$ The definition of iteration tree $\mathcal{T}$ in [24] differs slightly from here and from [4], in that $\operatorname{deg}^{\mathcal{T}}$ is not formally a component of $\mathcal{T}$. So in the terminology of [24], a tree can be both $k$-maximal and $j$-maximal, with $k \neq j$.
} 
a little notationally. A putative $k$-maximal tree $\mathcal{T}$ is a system satisfying the conditions of a $k$-maximal tree, except that if $\mathcal{T}$ has length $\alpha+1$ where $\alpha$ is a limit, then we do not demand that $[0, \alpha)_{\mathcal{T}} \cap \mathscr{D}^{\mathcal{T}}$ is finite (so $M_{\alpha}^{\mathcal{T}}$ is well-defined iff $[0, \alpha)_{\mathcal{T}} \cap \mathscr{D}^{\mathcal{T}}$ is finite), and if $\mathcal{T}$ has length $\beta+1$ and $M_{\beta}^{\mathcal{T}}$ is well-defined, we do not demand that $M_{\beta}^{\mathcal{T}}$ be wellfounded. ${ }^{13}$ See $\S 2$ for the particulars of (putative) $\mathrm{u}-m$-maximal trees.

Given an iteration tree $\mathcal{T}$ and $E=E_{\alpha}^{\mathcal{T}}$, we write $\widetilde{\nu}_{\alpha}^{\mathcal{T}}=\widetilde{\nu}(E)$ for the exchange ordinal associated to $E$ with respect to $\mathcal{T}$. So for $m$-maximal trees with $\lambda$ iteration rules on $\lambda$-indexed premice, $\widetilde{\nu}_{\alpha}^{\mathcal{T}}=\lambda(E)=\operatorname{lgcd}\left(\operatorname{ex} \mathcal{T}_{\alpha}^{\mathcal{T}}\right)$, whereas for $m$-maximal trees with MS-iteration rules on MS-indexed premice, $\widetilde{\nu}_{\alpha}^{\mathcal{T}}=\nu(E)$. However, we also deal with u-m-maximal trees (see §2), on MS-indexed premice or other seg-pms, where $\nu(E) \leq \widetilde{\nu}_{\alpha}^{\mathcal{T}} \leq \lambda(E)$, and strict inequalities are possible. And for coarse trees on wcpms, $\widetilde{\nu}_{\alpha}^{\mathcal{T}}=\varrho(E)$ (see $\left.\S 3\right)$.

Given a $q$-sound premouse $Q$ where $q \leq \omega$, a $q$-maximal stack on $Q$ is a sequence $\overrightarrow{\mathcal{T}}=\left\langle\mathcal{T}_{\alpha}\right\rangle_{\alpha<\lambda}$ of iteration trees such that for some $\left\langle Q_{\alpha}, q_{\alpha}, M_{\alpha}, m_{\alpha}\right\rangle_{\alpha<\lambda}$, $\mathcal{T}_{\alpha}$ is an $m_{\alpha}$-maximal tree on $M_{\alpha}, Q_{0}=Q, q_{0}=q,\left(M_{\alpha}, m_{\alpha}\right) \unlhd\left(Q_{\alpha}, q_{\alpha}\right)$, and if $\alpha+1<\lambda$ then $\mathcal{T}_{\alpha}$ has successor length and $Q_{\alpha+1}=M_{\infty}^{\mathcal{T}_{\alpha}}$ and $q_{\alpha+1}=\operatorname{deg}^{\mathcal{T}_{\alpha}}(\infty)$, and for limit $\eta<\lambda$, for all sufficiently large $\alpha<\eta,\left(M_{\alpha}, m_{\alpha}\right)=\left(Q_{\alpha}, q_{\alpha}\right), \mathcal{T}_{\alpha}$ does not drop on $b^{\mathcal{T}_{\alpha}}$, and $Q_{\eta}=M_{\infty}^{\overrightarrow{\mathcal{T}} \mid \eta}$ is the resulting direct limit of the $M_{\alpha}$ for $\alpha<\eta$ under the iteration maps and $q_{\eta}=\lim _{\alpha \rightarrow \eta} \operatorname{deg}^{\mathcal{T}_{\alpha}}(\infty)$. If $\lambda$ is a limit, we define $M_{\infty}^{\overrightarrow{\mathcal{T}}}$ and $\operatorname{deg}^{\overrightarrow{\mathcal{T}}}(\infty)$ as the natural direct limits, given that $\mathcal{T}_{\alpha}$ does not drop along $b^{\mathcal{T}_{\alpha}}$, etc, for all sufficiently large $\alpha$. We say an artificial drop occurs whenever $\left(M_{\alpha}, m_{\alpha}\right) \triangleleft\left(Q_{\alpha}, q_{\alpha}\right)$. An optimal stack is one without artificial drops. A putative $q$-maximal stack is as above, except that if it has length $\lambda=\alpha+1$, then $\mathcal{T}_{\alpha}$ is only required to be a putative tree. Likewise a (putative) $\mathrm{u}-q$-maximal stack on a $\mathrm{u}-q$-sound seg-pm.

The iteration game $\mathcal{G}(M, m, \alpha, \beta)^{*}$ of [23, p. 1202], ${ }^{14}$ consists of $\lambda \leq \alpha$ many rounds, producing a putative $m$-maximal stack $\left\langle\mathcal{T}_{\gamma}\right\rangle_{\gamma<\lambda}$ on $M$, with associated sequence $\left\langle Q_{\gamma}, q_{\gamma}, M_{\gamma}, m_{\gamma}\right\rangle_{\gamma<\lambda}$. In round $\gamma$, given $\left(Q_{\gamma}, q_{\gamma}\right)$, player I chooses $\left(M_{\gamma}, m_{\gamma}\right) \unlhd\left(Q_{\gamma}, q_{\gamma}\right)$, and then the players build the putative tree $\mathcal{T}_{\gamma}\left(m_{\gamma^{-}}\right.$ maximal, on $M_{\gamma}$ ), of length $\leq \beta$. If some model of $\mathcal{T}_{\gamma}$ is ill-defined or illfounded then $\lambda=\gamma+1$ and player I wins. Having produced a bona fide tree $\mathcal{T}_{\gamma} \uparrow(\xi+1)$, where $\xi+1<\beta$, player I may set $\mathcal{T}_{\gamma}=\mathcal{T}_{\gamma} \uparrow(\xi+1)$ and exit the round, and then $\lambda>\gamma+1$ (so round $\gamma+1$ will be played). If player I does not exit at any such stage $\xi+1<\beta$ and $\mathcal{T}_{\gamma}$ has wellfounded models then $\lambda=\gamma+1$ and player II wins. Given a limit $\gamma \leq \alpha$, player II must ensure that $M_{\infty}^{\overrightarrow{\mathcal{T}} \mid \gamma}$ is well-defined

\footnotetext{
${ }^{13}$ In a draft of this article on arxiv.org, the term putative (tree) precluded having infinitely many drops on a branch in the tree.

${ }^{14}$ In a draft of this paper which appeared on the preprint server arxiv.org, stacks were defined to be what we call optimal stacks here; thus, no artificial drops were considered in that draft. However, this is a more restrictive notion than Steel's definitions in [24], which do allow non-optimal stacks. We likewise stated that $\mathcal{G}(M, m, \alpha, \beta)^{*}$ was defined without permitting artificial drops, which is not consistent with [23]. Therefore, in that draft, we only constructed strategies for optimal stacks, not more generally. This oversight has now been amended, primarily through Lemma 9.8, but also see the proof of Theorem 9.6 in $\S 9.1 .3$, and the start of $\S 9.1 .4$, and the proof of Theorem 10.10 .
} 
and wellfounded; given this, if $\gamma=\alpha$ then player II wins, whereas if $\gamma<\alpha$ then $\lambda>\gamma$ and play continues.

For $\alpha$ a limit ordinal, the game $\mathcal{G}(M, m,<\alpha, \beta)^{*}$ has the same rules, except that if all $\alpha$ rounds are played through with no player having yet lost, then player II wins automatically, irrespective of whether $M_{\infty}^{\overrightarrow{\mathcal{T}}}$ is well-defined or wellfounded.

We define the optimal variants of these games, denoted $\mathcal{G}_{\text {opt }}(M, \alpha, \beta)^{*}$ and $\mathcal{G}_{\text {opt }}(M, m,<\alpha, \beta)^{*}$, with the same rules and payoffs as the games above, except that player I may not make artificial drops. So the optimal variants are superficially easier for player II. However, a straightforward copying argument, given in Lemma 9.8, which is much as in [17, §7], shows that if $\Sigma$ is a winning strategy for player II in $\mathcal{G}_{\text {opt }}(M, m, \alpha, \beta)^{*}$, then $\Sigma$ induces a canonical strategy $\Sigma^{\prime}$ for II in $\mathcal{G}(M, m, \alpha, \beta)^{*}$. Thus, in this paper, our main focus is on strategies for normal trees and for optimal stacks of normal trees.

\subsubsection{Embeddings}

For the definition of $n$-lifting embedding see [18, Definition 2.1].

Let $\pi: P \rightarrow Q$ be an embedding between seg-pms. We say that $\pi$ is cpreserving iff it is cardinal preserving, in that $\alpha$ is a cardinal of $P$ iff $\pi(\alpha)$ is a cardinal of $Q$. If $n=0$ or $P, Q$ are $(n-1)$-sound, we say that $\pi$ is $\vec{p}_{n}$-preserving iff $\pi\left(\vec{p}_{n}^{P}\right)=\vec{p}_{n}^{Q}$. We say that $\pi$ is nice $n$-lifting iff $\pi$ is $n$-lifting, c-preserving and $\vec{p}_{n}$-preserving. Note that every near $n$-embedding is nice $n$-lifting.

\section{$2 \mathrm{u}-m$-maximal iteration strategies}

The paper will deal with a lot of copying of iteration trees, requiring much associated bookkeeping. We deal with both kinds of premice - MS-indexed and $\lambda$-indexed - and also weak coarse premice. Recall that the standard copying algorithm does not quite work with type 3 MS-indexed premice. If we used here the standard fix to this problem (inserting extra extenders and slight modifications of tree order), we would need to integrate that fix into our bookkeeping, increasing notational and mental load. Fortunately, there is an alternate path, which we will adopt, which in the end allows us to separate the type 3 problem from the current bookkeeping. In this section we describe this path.

Whenever we say type $i$ premouse $M$, where $i \in\{0,1,2,3\}$, we mean that $M$ is MS-indexed. Everything in the present section is trivial for $\lambda$-indexed premice, and if the reader is happy to ignore the existence of type 3 premice, then they would have no problem ignoring the present section, as long as they replace all later instances of " $\mathrm{u}-m$ " with " $m$ ", where $m \leq \omega$, and as long as they imagine that all fine structural embeddings $\pi: M \rightarrow N$ between premice are such that $\operatorname{dom}(\pi)=M$ (not just $\left.M^{\mathrm{sq}}\right)$, and if $M$ is active then $\pi\left(\nu\left(F^{M}\right)\right)=\nu\left(F^{N}\right)$.

2.1 Remark. The prefix " $u$ " stands for unsquashed. It simply indicates that we compute fine structure, ultrapowers, etc, at the unsquashed level, with the active extender coded by the standard amenable predicate, just as is usually done for type 1 or 2 premice and $\lambda$-premice. Thus, for $\lambda$-premice and type 
$\leq 2$ premice, there is no difference between standard fine structure and " $\mathrm{u}$ " fine structure. For type 3, it represents a shift of 1 degree of complexity in the Levy hierarchy. However, because we also allow unsquashed ultrapowers, we also encounter seg-pms for which the Initial Segment Condition fails.

2.2 Definition. Let $n \leq \omega$ and let $M$ be a segmented-premouse. We say that $M$ is $\mathrm{u}-n$-sound iff either

1. $M$ is an $n$-sound premouse not of type 3 , or

2. $n \geq 2$ and $M$ is an (n-1)-sound type 3 premouse, where $\omega-1=\omega$, or

3. $n=1$ and $M$ is active and letting $\nu=\nu\left(F^{M}\right)$, there is an active type 3 premouse $M^{\prime}$ such that $\nu\left(F^{M^{\prime}}\right)=\nu$ and $F^{M^{\prime}}\left\lceil\nu=F^{M}\lceil\nu\right.$ and letting $\delta=\operatorname{lgcd}(M)$ and $U=\operatorname{Ult}\left(M^{\prime}, F^{M^{\prime}}\right)$, we have $M \| \mathrm{OR}^{M}=U \mid\left(\delta^{+}\right)^{U}$, or

4. $n=0$ and $\nu\left(F^{M}\right) \leq \operatorname{lgcd}(M)$.

Suppose $M$ is $\mathrm{u}-n$-sound. We say that $M$ is type $\mathbf{A}$ iff clause 1 above holds; otherwise we say that $M$ is type $\mathbf{B}$. If either $M$ is type $\mathrm{A}$, or $M$ is type $\mathrm{B}$ and $n \geq 2$, let $M^{\mathrm{pm}}=M$. If $M$ is type $\mathrm{B}$ and $n=1$ let $M^{\mathrm{pm}}=M^{\prime}$, as above. If $M$ is type $\mathrm{B}$, but not $\mathrm{u}$ - $n$-sound for any $n \geq 1$, then $M^{\mathrm{pm}}$ is not defined.

Let $M, N$ be $\mathrm{u}$ - $n$-sound segmented-premice and $\pi: M \rightarrow N$. Here the domain and codomain of $\pi$ are literally (the universes of) $M, N$, not $M^{\mathrm{sq}}, N^{\mathrm{sq}}$. We say that $\pi$ is a (near) $\mathrm{u}$ - $n$-embedding iff either:

1. $M, N$ are type $\mathrm{A}$ and $\pi$ is a (near) $n$-embedding, or

2. $M, N$ are type B and and $n \geq 1$ and $\pi^{\mathrm{sq}}=\pi \uparrow\left(M^{\mathrm{pm}}\right)^{\mathrm{sq}}: M^{\mathrm{pm}} \rightarrow N^{\mathrm{pm}}$ is a (near) $(n-1)$-embedding and $\pi$ is induced by $\pi^{\mathrm{sq}}$ and $\pi(\operatorname{lgcd}(M))=$ $\operatorname{lgcd}(N),{ }^{15}$ or

3. $M, N$ are type $\mathrm{B}$ and $n=0$ and $\pi$ is a (near) 0 -embedding ( $\pi$ is a near 0 embedding iff $\pi$ is $\mathrm{r} \Sigma_{1}$-elementary in the language of segmented-premice, and $\pi$ is a 0 -embedding iff $\pi$ is a near 0 -embedding and is cofinal in $\mathrm{OR}^{N}$ ).

The notion u- $n$-lifting embedding is defined by making analogous changes to the notion $n$-lifting embedding (defined in [18]).

2.3 Definition. For an active seg-pm $M, \widetilde{\nu}^{M}={ }_{\text {def }} \max \left(\nu\left(F^{M}\right), \operatorname{lgcd}(M)\right) . \quad \dashv$

Note that if $M, N$ are active and $\mathrm{u}-n$-sound and $\pi: M \rightarrow N$ is a (near) $\mathrm{u}-$ $n$-embedding then $\pi(\operatorname{lgcd}(M))=\operatorname{lgcd}(N)$ and $\pi\left(\widetilde{\nu}^{M}\right)=\widetilde{\nu}^{N}$. For $\pi(\operatorname{lgcd}(M))=$ $\operatorname{lgcd}(N)$ because $\pi$ respects the predicates for $F^{M}, F^{N}$. And if $M, N$ are type B then $\nu\left(F^{M}\right) \leq \operatorname{lgcd}(M)$ and $\nu\left(F^{N}\right) \leq \operatorname{lgcd}(N)$; therefore $\pi\left(\widetilde{\nu}^{M}\right)=\widetilde{\nu}^{N}$.

2.4 Definition. For a $\mathrm{u}-n$-sound seg-pm $M, \mathrm{u} \rho_{n}^{M}$ denotes $\rho$ where either:

- $M$ is type A and $\rho=\rho_{n}^{M}$, or

\footnotetext{
${ }^{15}$ Recall that the convention, for a fine structural embedding $\pi: M \rightarrow N$ between type 3 premice, is that literally $\pi: M^{\mathrm{sq}} \rightarrow N^{\mathrm{sq}}$.
} 
- $M$ is type $\mathrm{B}$ and $n \geq 1$ and $\rho=\rho_{n-1}^{M^{\mathrm{pm}}}$, or

- $M$ is type $\mathrm{B}$ and $n=0$ and $\rho=\mathrm{OR}^{M}$.

2.5 Definition. Let $M$ be a $\mathrm{u}-n$-sound seg-pm and let $E$ be a weakly amenable extender such that $\operatorname{cr}(E)<\mathrm{u} \rho_{n}^{M}$. Then $\operatorname{Ult}_{\mathrm{u}-n}(M, E)=U$ where either:

1. $M$ is type A and $U=\operatorname{Ult}_{n}(M, E)$, or

2. $M$ is type B and $n \geq 2$ and $U=\operatorname{Ult}_{n-1}(M, E)$, or

3. $M$ is type B and $n \leq 1$ and $U=\operatorname{Ult}(M, E)$ (so the ultrapower is direct; there is no squashing).

We also define $i_{E}^{M, \mathrm{u}-n}: M \rightarrow U$, abbreviated $i_{E}^{M}$, to be the (total) ultrapower map in cases 1 and 3 , or the (total) map it induces in case 2 .

The following lemma is a standard calculation:

2.6 Lemma. Let $M, E, n$ be as above, and suppose that $U=\operatorname{Ult}_{\mathrm{u}-n}(M, E)$ is wellfounded. Then $U$ is $\mathrm{u}-n$-sound and $i_{E}^{M}$ is a $\mathrm{u}$ - $n$-embedding.

2.7 Remark. In the definition of $\mathrm{Ult}_{\mathrm{u}-n}(M, E)$ above, the reader might expect that if $M$ is type $\mathrm{B}$ and $n=1$, it would be more natural to define the ultrapower using all functions which are $\sum_{1}^{M}$-definable, instead of just the functions in $M$. We digress to show that these two ultrapowers are equivalent (the content of this remark is not needed in the sequel).

Let $M$ be a type B with $n=1$. Write $\mathrm{u} \Sigma_{1}^{M}$ for the definability class over

$$
M=\left(\lfloor M\rfloor, \mathbb{E}^{M}, \widetilde{F^{M}}\right)
$$

itself, not its squash. Here $\widetilde{F^{M}}$ is the standard amenable coding of $F^{M}$. Let $\mathrm{u} \Sigma_{1}^{M}$ be the associated boldface class. By definition we have $\mathrm{u} \rho_{1}^{M}=\rho_{0}^{M}=\nu^{M}$. In fact, $\mathrm{u} \rho_{1}^{M}$ is the least $\rho$ such that there is a $\mathrm{u} \Sigma_{1}^{M}$ subset of $\rho$ not in $M$; see [8] or the proof of [12, Lemma 2.15]. Given $\eta<\mathrm{OR}^{M}$, let $M 2 \eta$ be the usual restriction of $M$ (with its predicates) to $M \| \eta$, that is,

$$
M \imath \eta=\left(M \| \eta, \mathbb{E}^{M}\left\lceil\eta, \widetilde{F^{M}} \cap(M \| \eta)\right) .\right.
$$

So the structures $M 2 \eta$ stratify $M$ as usual.

Suppose $\nu^{M}$ is regular in $M$ but $\mathrm{u} \sum_{1}^{M}$-singular, in the weak sense that there is some $\gamma<\nu^{M}$ and $x \in M$ such that $\operatorname{Hull}_{\mathrm{u} \Sigma}^{M}(\gamma \cup\{x\})$ is cofinal in $\nu^{M}$. Let $\gamma$ be least such. Note that there is $f: \gamma \rightarrow \nu^{M}$ which is $\underline{u} \Sigma_{1}^{M}$-definable, with $f$ " $\gamma$ cofinal in $\nu^{M}$ (that is, because of the characterization of $\mathrm{u} \rho_{1}^{M}=\nu^{M}$ just mentioned, we can recover a function $f$ with domain $\gamma$ ).

We claim $\gamma=\left(\mu^{+}\right)^{M}$ where $\mu=\operatorname{cr}\left(F^{M}\right)$. For we have the standard cofinal monotone increasing $\mathrm{u} \Sigma_{1}^{M}$ map $h:\left(\mu^{+}\right)^{M} \rightarrow \mathrm{OR}^{M}$ (derived from the amenable coding of $\left.F^{M}\right)$. Given $\alpha<\left(\mu^{+}\right)^{M}$, let $D_{\alpha} \subseteq \gamma$ be the set of all $\beta<\gamma$ such that $M \imath h(\alpha) \models$ " $f(\beta)$ is defined", and let $f_{\alpha}: D_{\alpha} \rightarrow \nu^{M}$ be the corresponding 
function. So $\gamma=\bigcup_{\alpha<\left(\mu^{+}\right)^{M}} D_{\alpha}$ and $f=\bigcup_{\alpha<\left(\mu^{+}\right)^{D}} f_{\alpha}$. But $f_{\alpha} \in M$, and since $\nu^{M}$ is $M$-regular, therefore $\operatorname{rg}\left(f_{\alpha}\right)$ is bounded in $\nu^{M}$. So defining $j:\left(\mu^{+}\right)^{M} \rightarrow$ $\nu^{M}$ by $j(\alpha)=\sup \operatorname{rg}\left(f_{\alpha}\right)$, then $j$ is $\mathrm{u} \Sigma_{1}^{M}$ and cofinal (and monotone increasing) in $\nu^{M}$. So $\gamma \leq\left(\mu^{+}\right)^{M}$. Also since $f \notin M$, there are cofinally many $\alpha, \beta<\left(\mu^{+}\right)^{M}$ such that $D_{\alpha} \subsetneq D_{\beta}$. But then since $\left\langle D_{\alpha}\right\rangle_{\alpha<\left(\mu^{+}\right)^{M}} \in M$ and $\left(\mu^{+}\right)^{M}$ is regular in $M$, we cannot have $\gamma<\left(\mu^{+}\right)^{M}$.

Now let $E$ be a weakly amenable $M$-extender with $\kappa=\operatorname{cr}(E)<\nu^{M}$ and $E_{a} \in M$ for all $a$ (because $M$ is type 3 , this will be the case for extenders $E$ applied to $M$ in a normal iteration tree). We claim that $\operatorname{Ult}(M, E)$ is equivalent to the ultrapower formed using all $\mathrm{u} \Sigma_{1}^{M}$ functions.

For this, let $f: \kappa^{|a|} \rightarrow M$ be a $\underline{u} \Sigma_{1}^{M}$ function. We want to see that there is $f^{\prime} \in M$ and $A \in E_{a}$ such that $f^{\prime}\left\lceil A=f\left\lceil A\right.\right.$. For $\eta<\left(\mu^{+}\right)^{M}$, let $f_{\eta}: D_{\eta} \rightarrow M$ be like before. So $f_{\eta} \in M$ and $f=\bigcup_{\eta<\left(\mu^{+}\right)^{M}} f_{\eta}$. If $\kappa \leq \mu$ then since $\left(\mu^{+}\right)^{M}$ is regular in $M$, there is $\eta$ such that $f=f_{\eta}$, which suffices. Suppose $\kappa>\mu$, so $\kappa>\left(\mu^{+}\right)^{M}$. Then $\left\langle D_{\eta}\right\rangle_{\eta<\left(\mu^{+}\right)^{M}} \in M$, and since $\bigcup_{\eta<\left(\mu^{+}\right)^{M}}=\kappa$, it follows that some $D_{\eta} \in E_{a}$, so $f_{\eta}, D_{\eta}$ works.

2.8 Definition. Let $k \leq \omega$ and $\lambda \in \mathrm{OR} \backslash\{0\}$ and let $M$ be a $\mathrm{u}$ - $k$-sound seg-pm. A u- $k$-maximal iteration tree $\mathcal{T}$ on $M$ of length $\lambda$ is a tuple

$$
\left(<^{\mathcal{T}}, \mathscr{D}, \mathrm{u}-\operatorname{deg},\left\langle M_{\alpha}\right\rangle_{\alpha<\lambda},\left\langle i_{\alpha \beta}, i_{\alpha \beta}^{*}\right\rangle_{\alpha, \beta<\lambda},\left\langle E_{\alpha}, \operatorname{ex}_{\alpha}, \widetilde{\nu}_{\alpha}, M_{\alpha+1}^{*}\right\rangle_{\alpha+1<\lambda}\right),
$$

such that:

1. $\mathscr{D} \subseteq \lambda$ and $\mathrm{u}-\operatorname{deg}: \lambda \rightarrow\{-1\} \cup(\omega+1)$.

2. $<^{\mathcal{T}}$ is an iteration tree order on $\lambda$.

3. $M_{0}=M$ and $0 \notin D$ and $\mathrm{u}-\operatorname{deg}(0)=k$.

4. For all $\beta<\lambda, M_{\beta}$ is a $\mathrm{u}-\operatorname{deg}(\beta)$-sound segmented-premouse.

5. For all $\alpha+1 \leq \beta+1<\lambda, \emptyset \neq E_{\alpha} \in \mathbb{E}_{+}^{M_{\alpha}}$ and $\operatorname{ex}_{\alpha}=M_{\alpha} \mid \operatorname{ind}\left(E_{\alpha}\right)$ and $\operatorname{ind}\left(E_{\alpha}\right) \leq \operatorname{ind}\left(E_{\beta}\right)$ and $\widetilde{\nu}_{\alpha}=\widetilde{\nu}^{\operatorname{ex}_{\alpha}}$.

6. For all $\alpha+1<\lambda$, letting $\kappa=\operatorname{cr}\left(E_{\alpha}\right)$ :

(a) $\beta=\operatorname{pred}^{\mathcal{T}}(\alpha+1)$ is the least $\xi$ such that $\kappa<\widetilde{\nu}_{\xi}$.

(b) $M_{\alpha+1}^{*}=$ least $N \unlhd M_{\beta}$ with $N=M_{\beta}$ or $\left[\operatorname{ex}_{\beta} \unlhd N\right.$ and $\left.\rho_{\omega}^{N} \leq \kappa\right]$.

(c) $\alpha+1 \in \mathscr{D}$ iff $M_{\alpha+1}^{*} \triangleleft M_{\beta}$.

(d) If $\alpha+1 \notin \mathscr{D}$ then $\mathrm{u}$ - $\operatorname{deg}(\alpha+1)=\operatorname{largest} n \leq \mathrm{u}$ - $\operatorname{deg}(\beta)$ with $\kappa<\mathrm{u} \rho_{n}^{M_{\beta}}$.

(e) If $\alpha+1 \in \mathscr{D}$ then $\mathrm{u}$ - $\operatorname{deg}(\alpha+1)=$ largest $n<\omega$ with $\kappa<\mathrm{u} \rho_{n}\left(M_{\alpha+1}^{*}\right)$.

(f) Let $n=\mathrm{u}-\operatorname{deg}(\alpha+1)$. Then $M_{\alpha+1}=\operatorname{Ult}_{\mathrm{u}-n}\left(M_{\alpha+1}^{*}, E_{\alpha}\right)$ and $i_{\alpha+1, \alpha+1}^{*}=$ $i_{E}^{M_{\alpha+1}^{*}, \mathrm{u}-n}$. Let $\gamma \leq^{\mathcal{T}} \beta$ with $(\gamma, \alpha+1] \cap \mathscr{D}=\emptyset$. Then

$$
i_{\gamma, \alpha+1}=i_{\alpha+1, \alpha+1}^{*} \circ i_{\gamma \beta},
$$

and if $\gamma$ is a successor then $i_{\gamma, \alpha+1}^{*}=i_{\alpha+1, \alpha+1}^{*} \circ i_{\gamma \beta}^{*}$. 
7. Let $\alpha \leq \mathcal{T} \gamma \leq^{\mathcal{T}} \beta<\lambda$ be such that $(\alpha, \beta]_{\mathcal{T}} \cap \mathscr{D}=\emptyset$. Then $i_{\alpha \beta}$ is defined and $i_{\alpha \beta}=i_{\gamma \beta} \circ i_{\alpha \gamma}$ and $\mathrm{u}-\operatorname{deg}(\beta) \leq \mathrm{u}-\operatorname{deg}(\alpha)$. (This condition follows from the others.)

8. Let $\eta<\lambda$ be a limit. Then there is $\alpha<^{\mathcal{T}} \eta$ with $(\alpha, \eta]_{\mathcal{T}} \cap \mathscr{D}=\emptyset$. Let $\alpha$ be least such and $m=\lim _{\beta<\tau} \eta \mathrm{u}-\operatorname{deg}(\beta)$. Then $m=\mathrm{u}-\operatorname{deg}(\eta)$ and

$$
M_{\eta}=\operatorname{dirlim}_{\beta \leq \gamma \in[\alpha, \eta)_{\mathcal{T}}}\left(M_{\beta}, M_{\gamma}, i_{\beta \gamma}\right),
$$

and for all $\beta \in[\alpha, \eta)_{\mathcal{T}}, i_{\beta \eta}$ is the associated direct limit map, and if also $\beta$ is a successor then $i_{\beta \eta}^{*}=i_{\beta \eta} \circ i_{\beta \beta}^{*}$.

The $\mathrm{u}$ - $k$-maximal iteration game $\mathcal{G}(M, \mathrm{u}-k, \theta),(\mathrm{u}-k, \theta)$-iteration strategy and $(\mathrm{u}-k, \theta)$-iterability are defined in the obvious manner. Likewise for stacks, such as the game $\mathcal{G}_{\text {opt }}(M, \mathrm{u}-k, \lambda, \theta)^{*}$, etc.

We say that $\mathcal{T}$ is a putative $\mathrm{u}-k$-maximal tree on $M$ iff all of the above properties hold, except that if $\lambda=\operatorname{lh}(\mathcal{T})$ is a successor, we do not require condition 4 to hold for $\beta=\lambda-1$, and if $\lambda-1$ is a limit, we do not require that $[0, \lambda-1)_{\mathcal{T}} \cap \mathscr{D}^{\mathcal{T}}$ is bounded in $\lambda-1$ (but if it is bounded, then we still define $M_{\lambda-1}^{\mathcal{T}}$ as before, etc).

It is routine to see that if $\mathcal{T}$ is a putative $\mathrm{u}$ - $k$-maximal tree of length $\eta+1$ and $M_{\eta}$ is well-defined and wellfounded, then $\mathcal{T}$ is a $\mathrm{u}-k$-maximal tree.

Moreover, if $\beta \leq^{\mathcal{T}} \eta$ and $(\beta, \eta]_{\mathcal{T}}$ does not drop in model then $i_{\beta \eta}^{\mathcal{T}}$ is a near $\mathrm{u}-m$-embedding, and if also $\mathrm{u}-\operatorname{deg}^{\mathcal{T}}(\beta)=\mathrm{u}-\operatorname{deg}^{\mathcal{T}}(\eta)$ then $i_{\beta \eta}^{\mathcal{T}}$ is a $\mathrm{u}-m$ embedding. Likewise for $i_{\beta \eta}^{* \mathcal{T}}$ if $\beta$ is also a successor.

2.9 Remark (Closeness for u). The Closeness Lemma [4, 6.1.5] adapts easily to $\mathrm{u}-m$-maximal trees on $\mathrm{u}-m$-sound MS-indexed seg-pms $M$. One key difference is that we replace the standard $\mathrm{r} \Sigma_{1}$ hierarchy with $\mathrm{u} \Sigma_{1}$ (see 2.7); of course, if $M$ is type $\leq 2$ then $\mathrm{r} \Sigma_{1}^{M}=\mathrm{u} \Sigma_{1}^{M}$. Thus, we say that an extender $E$ is uclose to a seg-pm $M$ iff $E$ is weakly amenable to $M$ and $E_{a}$ is $\underline{u} \Sigma_{1}^{M}$ for each $a \in[\nu(E)]^{<\omega}$. By 2.7 , if $M$ is a $\mathrm{u}$-1-sound premouse, so $M$ is equivalent to some type 3 premouse $N$, then $\mathrm{u} \rho_{1}^{M}=\nu^{N}$. As in [4], one shows that if $E$ is u-close to $M$ and $\mathrm{u} \rho_{1}^{M} \leq \operatorname{cr}(E)$ and $U=\mathrm{Ult}_{\mathrm{u}-0}(M, E)$, then $\mathrm{u} \rho_{1}^{U}=\mathrm{u} \rho_{1}^{M}$ and every $\mathrm{u} \Sigma_{1}^{U}$ subset of $\operatorname{cr}(E)$ is $\underline{\mathrm{u}} \Sigma_{1}^{M}$. As in [4, 6.1.5], one shows that if $\mathcal{T}$ is a $\mathrm{u}-m$-maximal tree on a $\mathrm{u}$ - $m$-sound seg-pm $M$, then $E_{\alpha}^{\mathcal{T}}$ is u-close to $M_{\alpha+1}^{* \mathcal{T}}$ for every $\alpha+1<\operatorname{lh}(\mathcal{T})$.

The proof of [9] adapts similarly, giving that the copying construction propagates near $\mathrm{u}$ - $m$-embeddings.

2.10 Definition. ${ }^{16}$ Let $M$ be a seg-pm with $M^{\text {pv }}$ MS-indexed, and $k^{\prime} \leq \omega$ with $M u$ u- $k^{\prime}$-sound. Suppose $M$ is type A or $k^{\prime} \geq 1$. If $M$ is type A or $k^{\prime}=\omega$

\footnotetext{
${ }^{16}$ In a draft of this article on arxiv.org, there is a version of the material in $2.10-2.16$ which is not quite correct in its treatment of translations of stacks, in that it does not restrict to the unravelled iteration game on the u-side (it also does not restrict to optimal stacks, though it is straightforward to handle this). That version is also not general enough to be applied to partial strategies (in particular in §9.1.4). The version here remedies these deficits.
} 
let $k=k^{\prime}$; otherwise let $k=k^{\prime}-1$. In this situation say $\left(M, k^{\prime}, k\right)$ is suitable. Let $\mathcal{T}$ be a $\mathrm{u}-k^{\prime}$-maximal tree on $M$.

Given $\alpha<\operatorname{lh}(\mathcal{T})$, say $\alpha$ is $\mathcal{T}$-special iff $M_{\alpha}^{\mathcal{T}}$ is $\mathcal{T}$-special iff $M_{\alpha}^{\mathcal{T}}$ is type B and $\mathrm{u}$ - $\operatorname{deg}^{\mathcal{T}}(\alpha)=0$. Say $\alpha$ is $\mathcal{T}$-very special (or $\mathcal{T}$-vs) iff $\alpha$ is $\mathcal{T}$-special and $E_{\alpha}^{\mathcal{T}}=F\left(M_{\alpha}^{\mathcal{T}}\right)$. Say $\alpha$ is a transition point of $\mathcal{T}$ iff $\alpha+1<\ln (\mathcal{T})$ and $\alpha$ is non- $\mathcal{T}$-special, but $\operatorname{OR}\left(\left(M_{\alpha}^{\mathcal{T}}\right)^{\mathrm{pm}}\right)<\operatorname{ind}\left(E_{\alpha}^{\mathcal{T}}\right)$ (note that in this situation, $M_{\alpha}^{\mathcal{T}}$ is type $\mathrm{B}$ and $\mathrm{u}-\operatorname{deg}^{\mathcal{T}}(\alpha)=1$, but $\left(M_{\alpha}^{\mathcal{T}}\right)^{\mathrm{pm}} \neq M_{\alpha}^{\mathcal{T}}$, so $M_{\alpha}^{\mathcal{T}}$ is not a premouse). Say $\mathcal{T}$ is unravelled iff, if $\mathcal{T}$ has successor length $\alpha+1$ then $\alpha$ is not $\mathcal{T}$-special.

The unravelling $\mathcal{S}=\operatorname{unrvl}(\mathcal{T})$ of $\mathcal{T}$, if it exists, is the unique unravelled u- $k^{\prime}$-maximal tree $\mathcal{S}$ on $M$ such that $\mathcal{T} \unlhd \mathcal{S}$, if $\mathcal{T}$ has limit length then $\mathcal{S}=\mathcal{T}$, and if $\operatorname{lh}(\mathcal{T})=\alpha+1$ then $\alpha+i$ is $\mathcal{S}$-vs for every $i$ such that $\alpha+i+1<\operatorname{lh}(\mathcal{S})$. Note that existence just requires wellfoundedness of the relevant models, and that if $\mathcal{S}$ exists, then $\ln (\mathcal{S})<\operatorname{lh}(\mathcal{T})+\omega$, because $\operatorname{cr}\left(E_{\alpha+i+1}^{\mathcal{S}}\right)<\operatorname{cr}\left(E_{\alpha+i}^{\mathcal{S}}\right)$.

Say $\mathcal{T}$ is everywhere unravelable iff $\operatorname{unrvl}(\mathcal{T}\lceil\alpha)$ exists (with wellfounded models) for all $\alpha \leq \operatorname{lh}(\mathcal{T})$, and for every transition point $\alpha$ of $\mathcal{T}$, $\operatorname{unrvl}\left(\mathcal{T}^{\prime}\right)$ exists where $\mathcal{T}^{\prime}=(\mathcal{T} \uparrow(\alpha+1))^{\wedge} F\left(M_{\alpha}^{\mathcal{T}}\right)$.

If $\overrightarrow{\mathcal{T}}=\left\langle\mathcal{T}_{\alpha}\right\rangle_{\alpha<\lambda}$ is an optimal $\mathrm{u}-k^{\prime}$-maximal stack on $M$, say $\overrightarrow{\mathcal{T}}$ is unravelled iff $\mathcal{T}_{\alpha}$ is unravelled for every $\alpha$, and say $\overrightarrow{\mathcal{T}}$ is everywhere unravelable iff $\mathcal{T}_{\alpha}$ is unravelled for each $\alpha+1<\lambda$ and $\mathcal{T}_{\alpha}$ is everywhere unravelable for each $\alpha<\lambda$.

The unravelled optimal u-iteration game $\mathcal{G}_{\mathrm{opt}}^{\mathrm{unrvl}}(M, \mathrm{u}-m, \alpha, \beta)^{*}$ is just like $\mathcal{G}_{\text {opt }}(M, \mathrm{u}-m, \alpha, \beta)^{*}$, except that player I may only round $\gamma$ with $\mathcal{T}_{\gamma}$ unravelled. This determines unravelled-optimal- $(\mathrm{u}-k, \alpha, \beta)^{*}$-iteration strategies and -iterability. For the corresponding definitions without the adjective optimal, there can be artificial drops as usual, but player I must still end rounds with unravelled trees.

2.11 Definition. Let $\left(M, k^{\prime}, k\right)$ be suitable. Let $\mathcal{U}$ be a $k$-maximal tree on $M^{\mathrm{pm}}$. Given $\alpha<\operatorname{lh}(\mathcal{U})$, define $M_{\alpha}^{+\mathcal{U}}$ as follows. (We stop if we reach an illfounded model. The notation is literally ambiguous, as it depends on $M$, whereas only $M^{\mathrm{pm}}$ is recorded in $\mathcal{U}$.) Set $M_{0}^{+\mathcal{U}}=M$. Let $\alpha+1<\operatorname{lh}(\mathcal{U})$ and $\beta=\operatorname{pred}^{\mathcal{U}}(\alpha+1)$. If $M_{\alpha+1}^{\mathcal{U}}$ is type $\leq 2$ or $\operatorname{deg}^{\mathcal{U}}(\alpha+1)=\omega$ let $m^{\prime}=\omega$; otherwise let $m^{\prime}=\operatorname{deg}^{\mathcal{U}}(\alpha+1)+1$. If $\alpha+1 \notin \mathscr{D}^{\mathcal{U}}$ let $N^{*}=M_{\beta}^{+\mathcal{U}}$; otherwise let $N^{*}=M_{\alpha+1}^{* \mathcal{U}}$. Now set $M_{\alpha+1}^{+\mathcal{U}}=\operatorname{Ult}_{\mathrm{u}-m^{\prime}}\left(N^{*}, E_{\alpha}^{\mathcal{U}}\right)$. Using the natural iteration maps

$$
i_{\alpha \beta}^{+\mathcal{U}}: M_{\alpha}^{+\mathcal{U}} \rightarrow M_{\beta}^{+\mathcal{U}}
$$

(defined when $\left.(\alpha, \beta]_{\mathcal{U}} \cap \mathscr{D}^{\mathcal{U}}=\emptyset\right)$, take direct limits at limit stages. We say $\mathcal{U}$ is $M$-u-wellfounded iff $M_{\alpha}^{+\mathcal{U}}$ is wellfounded for each $\alpha<\operatorname{lh}(\mathcal{U})$. Likewise for optimal $k$-maximal stacks $\overrightarrow{\mathcal{U}}=\left\langle\mathcal{U}_{\alpha}\right\rangle_{\alpha<\lambda}$, where $M_{0}^{+\mathcal{U}_{0}}=M$ and $M_{0}^{+\mathcal{U}_{\alpha}}=M_{\infty}^{+\overrightarrow{\mathcal{U}} \alpha}$ is the natural direct limit for $\alpha>0$; the stack is $M$-u-wellfounded iff every $M_{\beta}^{+\mathcal{U}_{\alpha}}$ is wellfounded.

The following two lemmas are proved in $[11, \S 4]$ : ${ }^{17}$

\footnotetext{
${ }^{17}$ Along with proving Lemmas 2.12 and 2.13, [11] describes a translation between $\lambda$-iteration rules and a natural version of MS-iteration rules for $\lambda$-indexed mice. The methods for both are similar. They are related to the proof of Theorem 5.8, and also to the methods of this
} 
2.12 Lemma. Let $\left(M, k^{\prime}, k\right)$ be suitable. There is a class bijection

$$
\mathcal{T} \mapsto \mathcal{U}=\operatorname{conv}(\mathcal{T})
$$

from the unravelled everywhere unravelable $\mathrm{u}-k^{\prime}$-maximal trees $\mathcal{T}$ on $M$ to the $M$-u-wellfounded $k$-maximal trees $\mathcal{U}$ on $M^{\mathrm{pm}}$, such that:

1. If $\mathcal{S} \unlhd \mathcal{U}$ then either

- $\mathcal{S}=\operatorname{conv}(\operatorname{unrvl}(\mathcal{T}\lceil\alpha))$ for some $\alpha$, or

- $\mathcal{S}=\operatorname{conv}\left(\operatorname{unrvl}\left(\mathcal{T}\left\lceil(\alpha+1) \wedge F\left(M_{\alpha}^{\mathcal{T}}\right)\right)\right)\right.$ for a transition point $\alpha$ of $\mathcal{T}$.

2. $\ln (\mathcal{T})$ is a limit iff $\ln (\mathcal{U})$ is a limit. When limits, these lengths are equal.

3. Suppose $\operatorname{lh}(\mathcal{T})=\alpha^{\prime}+1$ and $\operatorname{lh}(\mathcal{U})=\alpha+1$. Then:

(a) $\left(M_{\alpha^{\prime}}^{\mathcal{T}}\right)^{\mathrm{pm}}=M_{\alpha}^{\mathcal{U}}$ and $M_{\alpha^{\prime}}^{\mathcal{T}}=M_{\alpha}^{+\mathcal{U}}$, so if $M_{\alpha}^{\mathcal{U}}$ is non-type 3 or $\operatorname{deg}^{\mathcal{U}}(\alpha)>$ 0 then $M_{\alpha^{\prime}}^{\mathcal{T}}=M_{\alpha}^{\mathcal{U}}$

(b) either $M_{\alpha^{\prime}}^{\mathcal{T}}=M_{\alpha}^{\mathcal{U}}$, or $\left(M_{\alpha}^{\mathcal{U}}\right)^{\mathrm{pv}} \triangleleft M_{\alpha^{\prime}}^{\mathcal{T}}$ and $\mathrm{OR}^{M_{\alpha}^{\mathcal{U}}}$ is an $M_{\alpha^{\prime}}^{\mathcal{T}}$-cardinal,

(c) $\left[0, \alpha^{\prime}\right]_{\mathcal{T}} \cap \mathscr{D}^{\mathcal{T}}=\emptyset$ iff $[0, \alpha]_{\mathcal{U}} \cap \mathscr{D}^{\mathcal{U}}=\emptyset$; likewise for $\mathscr{D}_{\operatorname{deg}}^{\mathcal{T}}$ and $\mathscr{D}_{\operatorname{deg}}^{\mathcal{U}}$,

(d) letting $\beta^{\prime}+1 \leq^{\mathcal{T}} \alpha^{\prime}$ and $\beta+1 \leq^{\mathcal{U}} \alpha$ be least such that $\left(\beta^{\prime}+1, \alpha^{\prime}\right]_{\mathcal{U}}$ and $(\beta+1, \alpha]_{\mathcal{T}}$ do not drop in model or degree, then:

$$
\left(M_{\beta^{\prime}+1}^{* \mathcal{T}}\right)^{\mathrm{pm}}=M_{\beta+1}^{* \mathcal{U}} \text { and } i_{\beta^{\prime}+1, \alpha^{\prime}}^{* \mathcal{T}}\left\lceil\left(\left(M_{\beta^{\prime}+1}^{* \mathcal{T}}\right)^{\mathrm{pm}}\right)^{\mathrm{sq}}=i_{\beta+1, \alpha}^{* \mathcal{U}},\right.
$$

and in fact if $[0, \alpha]_{\mathcal{U}} \cap \mathscr{D}_{\mathrm{deg}}^{\mathcal{U}} \neq \emptyset$ then $M_{\beta^{\prime}+1}^{* \mathcal{T}}=M_{\beta+1}^{* \mathcal{U}}$.

Further, there is an analogous bijection between unravelled everywhere unravelable optimal $k^{\prime}$-maximal stacks $\left\langle\mathcal{T}_{\alpha}\right\rangle_{\alpha<\lambda}$ on $M$ and $M$-u-wellfounded optimal $k$-maximal stacks $\left\langle\mathcal{U}_{\alpha}\right\rangle_{\alpha<\lambda}$ on $M^{\mathrm{pm}}$. Moreover, $\mathcal{U}_{\alpha}=\operatorname{conv}\left(\mathcal{T}_{\alpha}\right)$ for each $\alpha$.

The bijections are moreover uniformly definable from the parameter $M$. If $M$ is countable then the conversion between such $\mathcal{U}, \mathcal{T} \in \mathrm{HC}$ is $\Delta_{1}^{1}(\{M\})$-definable in the codes, and likewise for optimal stacks.

If we at times talk about the conversion of an everywhere unravelable u- $k^{\prime}$ maximal tree $\mathcal{T}$ to a $k$-maximal tree, without assuming that $\mathcal{T}$ is unravelled, then one should first replace $\mathcal{T}$ with $\operatorname{unrvl}(\mathcal{T})$.

2.13 Lemma. Let $\Omega>\omega$ be regular and $\Omega \leq \Xi \leq \Omega+1$. Let $\left(M, k^{\prime}, k\right)$ be suitable, with $M$ a premouse. Then

1. $M$ is (u- $\left.k^{\prime}, \Xi\right)$-iterable iff $M$ is $(k, \Xi)$-iterable,

2. $M$ is unravelled-opt-(u- $\left.k^{\prime}, \Omega, \Xi\right)^{*}$-iterable iff $M$ is opt- $(k, \Omega, \Xi)^{*}$-iterable.

Moreover, there are bijections $\Sigma \mapsto \operatorname{conv}(\Sigma)$ between the sets of

3. (u- $\left.k^{\prime}, \Xi\right)$-strategies and $(k, \Xi)$-strategies,

paper more generally. We have written $\mathcal{T}^{\prime} \mapsto \mathcal{T}$ here, instead of $\mathcal{T} \mapsto \mathcal{T}^{\prime}$, to match better with the notation in [11]. 
4. unravelled-opt-(u- $\left.k^{\prime}, \Omega, \Xi\right)^{*}$-strategies and opt- $(k, \Omega, \Xi)^{*}$-strategies,

5. unravelled-opt- $\left(\mathrm{u}-k^{\prime},<\omega, \Omega\right)^{*}$-strategies and opt- $(k,<\omega, \Omega)^{*}$-strategies

for $M$. In particular, there is a unique $\left(\mathrm{u}-k^{\prime}, \Xi\right)$-strategy for $M$ iff there is a unique $(k, \Xi)$-strategy for $M$.

These bijections are induced tree-by-tree, for unravelled trees via $\Sigma$ and trees via $\Gamma=\operatorname{conv}(\Sigma)$, via the correspondence of Lemma 2.13, and therefore if $\Omega=\aleph_{1}$ and $\widetilde{\Sigma}$ is the natural coding of $\Sigma \uparrow$ HC over $\mathbb{R}$, and $\widetilde{\Gamma}$ likewise, then $\widetilde{\Gamma}$ is $\Delta_{1}^{1}(\widetilde{\Sigma})$ and vice versa.

2.14 Remark. Note here that if $\Sigma$ is a $(\mathrm{u}-k, \Xi)$-strategy, then all trees via $\Sigma$ are everywhere unravelable. Similarly, if $\Gamma$ is a $(k, \Xi)$-strategy for $M$, then all trees via $\Gamma$ are in fact $M$-u-wellfounded. (If $\Xi=\Omega+1$, then as $\Omega$ is regular, $\Sigma$ in fact extends to a $(\mathrm{u}-k, \Omega+\omega)$-strategy. So unravellings of trees via $\Sigma$ always exist. Similarly for $\Gamma$.) So Lemma 2.12 (and its proof) is relevant to the proof of Lemma 2.13.

At times we will also deal with partial strategies (where the trees in the domain of the strategy have some restricted form).

2.15 Definition. A partial strategy $\Sigma$ for $\mathrm{u}-k^{\prime}$-maximal trees/stacks is everywhere unravelable if all trees via $\Sigma$ are everywhere unravelable. A partial strategy $\Gamma$ for an MS-indexed premouse $M$ for $k$-maximal trees/stacks is $M$ u-wellfounded if all trees via $\Gamma$ are $M$-u-wellfounded.

2.16 Remark. Note that if $\Gamma$ (as above) is $M$-u-wellfounded, then we can define via Lemma 2.12 a partial u-strategy $\Sigma$, where the trees via $\Sigma$ are just those which are initial segments of trees $\mathcal{T}=\operatorname{conv}^{-1}(\mathcal{U})$ for some $\mathcal{U}$ via $\Gamma$ (and if a strategy for stacks, then we admit only stacks according to the unravelled game); all putative trees via $\Sigma$ are then true trees (and are everywhere unravelable). Likewise conversely, if a given $\Sigma$ (as above) is everywhere unravelable, then we can define the corresponding partial strategy $\Gamma$, and all trees via $\Gamma$ are $M$ u-wellfounded.

\section{Coarse mice}

The main results and methods in the paper also apply to iteration strategies for a natural class of coarse structures. Steel suggested to the author that the methods should go through in such a context, and it was indeed straightforward to verify that things go through with the same basic ideas, and with some simplifications. The only slight subtlety is that we seem to need a weak form of a coherent sequence of extenders for some of the arguments (such a notion was already employed by Steel in his work). The coarse case will be used by Steel and the author in the forthcoming paper [19].

3.1 Definition. A weak coarse premouse (wcpm) is a transitive structure $M=\left(N, \delta, \mathbb{E},<_{e}\right)$ such that: 
$-\delta \leq \mathrm{OR}^{N}=\operatorname{rank}(N), \delta$ and $\mathrm{OR}^{N}$ are limit ordinals, $\operatorname{card}^{N}\left(V_{\eta}^{N}\right)<\delta$ for every $\eta<\delta, \operatorname{cof}^{N}(\delta)$ is not measurable in $N, N$ satisfies $\Sigma_{0}$-comprehension and is rudimentarily closed, and $N$ satisfies $\lambda$-choice for all $\lambda<\delta$.

- $\mathbb{E},<_{e} \subseteq V_{\delta}^{N}$ and both are amenable to $V_{\delta}^{N}$.

- $\mathbb{E}$ is a class of $E$ such that $N \models " E$ is a suitable extender".

$-<_{e}$ is a wellorder of $\mathbb{E}$.

- if $E, F \in \mathbb{E}$ and $\varrho^{N}(E)<\varrho^{N}(F)$ then $E<_{e} F$.

Given a wcpm $M=\left(N, \delta, \mathbb{E},<_{e}\right)$ and $E \in \mathbb{E}$, then $\operatorname{Ult}(M, E)$ denotes $\left(\operatorname{Ult}(N, E), \delta^{\prime}, \mathbb{E}^{\prime},<_{e}^{\prime}\right)$ where $\delta^{\prime}=i_{E}^{N}(\delta)$,

$$
\begin{aligned}
& \mathbb{E}^{\prime}=\bigcup_{\alpha<\delta} i_{E}^{N}\left(\mathbb{E} \cap V_{\alpha}^{N}\right), \\
& <_{e}^{\prime}=\bigcup_{\alpha<\delta} i_{E}^{N}\left(<_{e} \cap V_{\alpha}^{N}\right) .
\end{aligned}
$$

Given a wcpm $M$ and $E \in \mathbb{E}^{M}$, we write ind $(E)$ (or ind $\left.{ }^{M}(E)\right)$ for the ordinal rank of $E$ in $<_{e}^{M}$.

Given a wcpm $M$, we say that $M$ is slightly coherent iff for every $E \in \mathbb{E}$, letting $\varrho=\varrho^{M}(E)$ and $U=\operatorname{Ult}(M, E)$, we have:

1. $X=_{\text {def }}\left\{F \in \mathbb{E}^{M} \mid \varrho^{M}(F)<\varrho\right\}=\left\{F \in \mathbb{E}^{U} \mid \varrho^{U}(F)<\varrho\right\}$,

2. $<_{e}^{M} \uparrow X=<_{e}^{U} \uparrow X$,

3. for each $F \in \mathbb{E}^{U}$, if $\varrho^{U}(F)=\varrho$ then $F \in \mathbb{E}^{M}$ and $F<_{e}^{M} E$.

3.2 Remark. We need slight coherence for the normal realization results in $\S 9$ and genericity inflation in $\S 5$. For the other results, slight coherence is not relevant.

3.3 Definition. We write $\mathcal{L}_{\mathrm{LST}}$ for the language of set theory, and $\mathcal{L}_{\mathrm{LST}}^{+}$for $\mathcal{L}_{\text {LST }}$ augmented with 1 -place predicates $\mathbb{E}$ and $<_{e}$.

Let $M, N$ be wcpms and $\pi: M \rightarrow N$. We say $\pi$ is a coarse 0 -embedding iff:

- $\pi$ is $\in$-cofinal in $N$,

- $\pi \uparrow V_{\delta^{M}}^{M}$ is $\in$-cofinal in $V_{\delta^{N}}^{N}$,

- $\pi$ is $\Sigma_{1}$-elementary in $\mathcal{L}_{\mathrm{LST}}$, and

- $\pi \uparrow V_{\delta^{M}}^{M}:\left(V_{\delta^{M}}^{M}, \mathbb{E}^{M},<_{e}^{M}\right) \rightarrow\left(V_{\delta^{N}}^{N}, \mathbb{E}^{N},<_{e}^{N}\right)$ is $\Sigma_{1}$ elementary in $\mathcal{L}_{\mathrm{LST}}^{+} . \quad \dashv$

3.4 Lemma. Let $M$ be a wcpm. Then each proper segment of $<_{e}^{M}$ is in $V_{\delta^{M}}^{M}$, and $<_{e}^{M}$ has ordertype $\leq \delta^{M}$. 
Proof. For each $\varrho<\delta^{M}$, the set $\left\{E \in \mathbb{E}^{M} \mid \varrho^{M}(E) \leq \varrho\right\} \in V_{\delta^{M}}^{M}$, because $\operatorname{card}^{M}\left(V_{\eta}^{M}\right)<\delta^{M}$ for every $\eta<\delta^{M}$ and every $E \in \mathbb{E}^{M}$ is suitable. Since $<_{e}^{M}$ refines strength and by the amenability of it and $\mathbb{E}^{M}$, this gives the lemma.

3.5 Lemma. Let $M$ be a wcpm. Let $E$ be a short $M$-extender with $\operatorname{cr}(E)$ measurable in $M$ and $U=\operatorname{Ult}(M, E)$ wellfounded. Then: (i) $\Sigma_{0}$-Łoś' Theorem holds for $\mathcal{L}_{\mathrm{LST}}$, (ii) $\Sigma_{0}$-Loś' Theorem holds for $\mathcal{L}_{\mathrm{LST}}^{+}$with respect to parameters in $V_{\delta^{M}}^{M}$, (iii) $U$ is a wcpm, (iv) $i_{E}^{M}: M \rightarrow U$ is a coarse 0-embedding, (v) If $M$ is slightly coherent then so is $U$.

3.6 Definition. Let $M$ be a wcpm. A normal iteration tree $\mathcal{T}$ on $M$ is defined in a typical manner, with the specific requirements that for all $\alpha+1<$ $\operatorname{lh}(\mathcal{T})$, we have:

- $M_{\alpha}^{\mathcal{T}}$ is a wcpm and $E_{\alpha}^{\mathcal{T}} \in \mathbb{E}^{M_{\alpha}^{\mathcal{T}}}$; we write $\varrho_{\alpha}^{\mathcal{T}}=\varrho^{M_{\alpha}^{\mathcal{T}}}\left(E_{\alpha}^{\mathcal{T}}\right)$,

- If $\beta+1<\alpha+1$ then $\varrho_{\beta}^{\mathcal{T}}<\varrho_{\alpha}^{\mathcal{T}}$.

$-\operatorname{pred}^{\mathcal{T}}(\alpha+1)$ is the least $\beta$ such that $\operatorname{cr}\left(E_{\alpha}^{\mathcal{T}}\right)<\varrho_{\beta}^{\mathcal{T}}$.

A putative normal iteration tree on $M$ is just like a normal tree on $M$, except that if $\mathcal{T}$ has successor length $\alpha+1>1$ then we do not demand that $M_{\alpha}^{\mathcal{T}}$ be a wcpm (nor wellfounded).

The following lemma is verified by a routine induction:

3.7 Lemma. Let $\mathcal{T}$ be a putative normal iteration tree on the wcpm $M$. If $\mathcal{T}$ has wellfounded models, then its models are wcpms, so $\mathcal{T}$ is a normal tree.

Now suppose that $\mathcal{T}$ is a normal iteration tree. Then for every $\alpha<\operatorname{lh}(\mathcal{T})$, writing $M_{\alpha}=M_{\alpha}^{\mathcal{T}}$ etc,

1. If $\beta<\mathcal{T} \alpha$ then $i_{\beta \alpha}^{\mathcal{T}}: M_{\beta} \rightarrow M_{\alpha}$ is cofinal and $\Sigma_{1}$-elementary in $\mathcal{L}_{\mathrm{LST}}$.

2. If $\beta<\mathcal{T} \alpha$ then $i_{\beta \alpha}^{\mathcal{T}}:\left(V_{\delta^{M^{\beta}}}^{M_{\beta}}, \mathbb{E}^{M_{\beta}},<_{e}^{M_{\beta}}\right) \rightarrow\left(V_{\delta^{M_{\alpha}}}^{M_{\alpha}}, \mathbb{E}^{M_{\alpha}},<_{e}^{M_{\alpha}}\right)$ is cofinal and $\Sigma_{1}$-elementary in $\mathcal{L}_{\mathrm{LST}}^{+}$.

3. Suppose $M$ is slightly coherent. Then so is $M_{\alpha}$, and for $\beta<\alpha$, letting $\varrho=\varrho_{\beta}^{\mathcal{T}}$, we have:

$$
\begin{aligned}
& -X={ }_{\operatorname{def}}\left\{F \in \mathbb{E}^{M_{\beta}} \mid \varrho^{M_{\beta}}(F)<\varrho\right\}=\left\{F \in \mathbb{E}^{M_{\alpha}} \mid \varrho^{M_{\alpha}}(F)<\varrho\right\}, \\
& -<_{e}^{M_{\beta}}\left\lceil X=<_{e}^{M_{\alpha}}\lceil X,\right. \\
& \text { - for each } F \in \mathbb{E}^{M_{\alpha}}, \text { if } \varrho^{M_{\alpha}}(F)=\varrho \text { then } F \in \mathbb{E}^{M_{\beta}} \text { and } F<_{e}^{M_{\beta}} E_{\beta}^{\mathcal{T}} .
\end{aligned}
$$

3.8 Definition. We define (normal) $\alpha$-iteration strategies and $\alpha$-iterability (where $\alpha \in \mathrm{OR}$ ) for a wcpm $M$ in the obvious manner. Likewise stacks of normal trees, $(\lambda, \alpha)^{*}$-iteration strategies and $(\lambda, \alpha)^{*}$-iterability (in which $\lambda$ is the length of the stack, and $\alpha$ the bound on the length of the individual normal trees; player I may stop round before reaching a normal tree of length $\alpha$, and otherwise the game terminates; if $\lambda$ is a limit then player II must also ensure that the direct limit $M_{\infty}^{\overrightarrow{\mathcal{T}}}$ of the entire stack $\overrightarrow{\mathcal{T}}$ is wellfounded). 
3.9 Definition. Given a wcpm $M$, we write $\mathbb{E}_{+}(M)=\mathbb{E}_{+}^{M}=\mathbb{E}(M)=\mathbb{E}^{M}$ (cf. the use of $\mathbb{E}, \mathbb{E}_{+}$in connection with seg-pms).

\section{Tree embeddings and inflation}

In this section we introduce the key concepts of the paper: tree embeddings, inflation, and various kinds of condensation for iteration strategies to which these notions lead. These notions were introduced somewhat in $\S 1$. But first we lay down some iteration tree terminology; see $\S 1.1$ for more.

\subsection{Iteration tree terminology}

4.1 Definition. Let $M$ be an active seg-pm and $\delta=\operatorname{lgcd}(M)$. We define $\iota^{M}=\iota(M)$. If $\nu\left(F^{M}\right) \leq \delta$ and $\delta$ is a limit cardinal of $M$ then $\iota^{M}=\delta$; otherwise $\iota^{M}=\mathrm{OR}^{M}$. For an iteration tree $\mathcal{T}$ and $\alpha+1<\operatorname{lh}(\mathcal{T}), \iota_{\alpha}^{\mathcal{T}}$ denotes $\iota\left(\operatorname{ex}_{\alpha}^{\mathcal{T}}\right) .{ }^{18} \dashv$

4.2 Remark. Let $\mathcal{T}$ be an $m$-maximal or $\mathrm{u}-m$-maximal tree (on a seg-pm with either indexing). Recall that $\widetilde{\nu}_{\alpha}^{\mathcal{T}}$ is the exchange ordinal associated to $E_{\alpha}^{\mathcal{T}}$. However, note that we could have used $\iota_{\alpha}^{\mathcal{T}}$ instead, without changing the tree order. Moreover, in the tree copying we will do, if $\sigma: M_{\alpha}^{\mathcal{T}} \rightarrow M_{\alpha^{\prime}}^{\mathcal{T}^{\prime}}$ is a copy map and $E_{\alpha^{\prime}}^{\mathcal{T}^{\prime}}$ is the lift of $E_{\alpha}^{\mathcal{T}}$ (under $\sigma$ ) then $\sigma\left\lceil\iota_{\alpha}^{\mathcal{T}}\right.$ will agree with later copy maps. (But there will be instances where $\iota_{\alpha}^{\mathcal{T}}<\mathrm{OR}\left(\operatorname{ex}_{\alpha}^{\mathcal{T}}\right)$ but $\sigma\left\lceil\mathrm{OR}\left(\operatorname{ex}_{\alpha}^{\mathcal{T}}\right)\right.$ does not agree with later copy maps.)

4.3 Definition. Let $\mathcal{T}$ be an iteration tree, $\eta=\operatorname{lh}(\mathcal{T})$ and suppose $\mathcal{T}$ is either:

(i) a normal tree on the wcpm $M$, or

(ii) a $\mathrm{u}-m$-maximal tree on the $\mathrm{u}$ - $m$-sound seg-pm $M$, or

(iii) an $m$-maximal tree on the $m$-sound $\mathrm{pm} M$.

If $\eta$ is a limit and $b$ is a $\mathcal{T}$-cofinal branch, we write $(\mathcal{T}, b)$ or $\mathcal{T}^{\wedge} b$ for the putative tree $\mathcal{T}^{\prime}$ extending $\mathcal{T}$, of length $\eta+1$, with $[0, \eta)_{\mathcal{T}^{\prime}}=b$.

Suppose $\eta=\beta+1$. For $E \in \mathbb{E}_{+}\left(M_{\beta}^{\mathcal{T}}\right)$, we say that $E$ is $\mathcal{T}$-normal iff either

- (ii) or (iii) above holds and $\operatorname{ind}\left(E_{\alpha}^{\mathcal{T}}\right) \leq \operatorname{ind}(E)$ for all $\alpha<\beta$, or

- (i) above holds and $\varrho_{\alpha}^{\mathcal{T}}<\varrho^{M_{\beta}^{\mathcal{T}}}(E)$ for all $\alpha<\beta$.

If $E$ is $\mathcal{T}$-normal, then $\mathcal{T}^{\wedge}\langle E\rangle$ denotes the putative tree $\mathcal{T}^{\prime}$ extending $\mathcal{T}$, of length $\eta+1$, such that either (i) $\mathcal{T}^{\prime}$ is $\mathrm{u}$ - $m$-maximal, or (ii) $\mathcal{T}^{\prime}$ is $m$-maximal, or (iii) $\mathcal{T}^{\prime}$ is normal, respectively according to the case for $\mathcal{T}$ above. ${ }^{19}$

\footnotetext{
${ }^{18}$ Recall that $\operatorname{ex}_{\alpha}^{\mathcal{T}}=M_{\alpha}^{\mathcal{T}} \mid \operatorname{ind}\left(E_{\alpha}^{\mathcal{T}}\right)$.

${ }^{19}$ We take it that the basic fine structural information regarding an iteration tree $\mathcal{U}$ is explicitly given with $\mathcal{U}$, so there can be no ambiguity here.
} 
4.4 Definition (Model dropdown). Let $M$ be a putative ${ }^{20} \mathrm{u}$ - $k$-sound seg-pm and $\lambda \leq \mathrm{OR}^{M}$, where if $M$ is illfounded then $\lambda=\mathrm{OR}^{M}$. The extended model dropdown sequence of $(M, \lambda)$ is the sequence $\left\langle M_{i}\right\rangle_{i \leq n}$ of maximal length such that $M_{0}=M \mid \lambda$, and given $M_{i} \triangleleft M, M_{i+1}$ is the least $N \unlhd M$ such that either (i) $N=M$ or (ii) $M_{i} \triangleleft N$ and $\rho_{\omega}^{N}<\rho_{\omega}^{M_{i}}$. The reverse of a sequence $\left\langle N_{i}\right\rangle_{i \leq n}$ (where $n<\omega$ ) is $\left\langle N_{n-i}\right\rangle_{i \leq n}$.

4.5 Definition (Tree dropdown). Let $M$ be a u- $k$-sound segmented-premouse and let $\mathcal{T}$ be a putative $\mathrm{u}$ - $k$-maximal tree on $M$.

For $\beta+1<\operatorname{lh}(\mathcal{T})$ let $\lambda_{\beta}=\operatorname{ind}\left(E_{\beta}^{\mathcal{T}}\right)$. For $\beta+1=\operatorname{lh}(\mathcal{T})($ if $\operatorname{lh}(\mathcal{T})$ is a successor) let $\lambda_{\beta}=\operatorname{OR}\left(M_{\beta}^{\mathcal{T}}\right)$. Let $\beta<\operatorname{lh}(\mathcal{T})$. Let $\left\langle M_{\beta i}\right\rangle_{i \leq m_{\beta}}$ be the reversed extended model dropdown sequence of $\left(M_{\beta}^{\mathcal{T}}, \lambda_{\beta}\right)$ (this defines $m_{\beta}$ ). Here if $M_{\beta}^{\mathcal{T}}$ is ill-defined, set instead $\lambda_{\beta}=m_{\beta}=M_{\beta 0}^{\mathcal{T}}=0$. Then $m_{\beta}^{\mathcal{T}}={ }_{\text {def }} m_{\beta}$ and $M_{\beta i}^{\mathcal{T}}={ }_{\text {def }} M_{\beta i}$. Let $\theta \leq \operatorname{lh}(\mathcal{T})$. We define the dropdown domain $\operatorname{ddd}^{(\mathcal{T}, \theta)}$ of $(\mathcal{T}, \theta)$ by

$$
\Delta=\operatorname{ddd}^{(\mathcal{T}, \theta)}={ }_{\operatorname{def}}\left\{(\beta, i) \mid \beta<\theta \& i \leq m_{\beta}\right\},
$$

and the dropdown sequence dds ${ }^{(\mathcal{T}, \theta)}$ of $(\mathcal{T}, \theta)$ by dds ${ }^{(\mathcal{T}, \theta)}=\operatorname{def}_{\operatorname{def}}\left\langle M_{\beta i}\right\rangle_{(\beta, i) \in \Delta}$.

The dropdown sequence $\mathrm{dds}^{\mathcal{T}}$ of $\mathcal{T}$ is $\mathrm{dds}^{(\mathcal{T}, \operatorname{lh}(\mathcal{T}))}$, and the dropdown domain $\operatorname{ddd}^{\mathcal{T}}$ of $\mathcal{T}$ is $\operatorname{ddd}^{(\mathcal{T}, \operatorname{lh}(\mathcal{T}))}$.

4.6 Definition. Let $\mathcal{X}$ be an iteration tree. Then $\operatorname{clint}^{\mathcal{X}}$ denotes the set of closed $<^{\mathcal{X}}$-intervals.

\subsection{Tree embeddings}

We now define the notion of a tree embedding $\Pi: \mathcal{T} \hookrightarrow \mathcal{X}$ between normal trees $\mathcal{T}, \mathcal{X}$ (actually we allow $\mathcal{T}$ to be a putative tree). This is fairly straightforward, but there are a lot of details to keep track of, reminiscent of iterability proofs with resurrection. We first roughly describe the objects involved, to give an idea of what to expect. The primary data determining the tree embedding is an embedding of the tree structure of $\mathcal{T}$ into that of $\mathcal{X}$. This embedding will determine canonical copy embeddings from models in the dropdown sequence of $\mathcal{T}$ to initial segments of models of $\mathcal{X}$. A natural degree of commutativity between the copy embeddings and iteration embeddings will be required. For each extender used in $\mathcal{T}$ there will be a corresponding copy of this extender used in $\mathcal{X}$. A key point is that, corresponding to each $\beta<\operatorname{lh}(\mathcal{T})$, we will typically have not just a single corresponding node in $\mathcal{X}$, but a corresponding $\mathcal{X}$-interval $I_{\beta}=\left[\gamma_{\beta}, \delta_{\beta}\right]_{\mathcal{X}}$. We will have a copy embedding

$$
\pi_{\beta 0}: M_{\beta}^{\mathcal{T}} \rightarrow M_{\gamma_{\beta}}^{\mathcal{X}}
$$

(with codomain $M_{\gamma_{\beta}}^{\mathcal{X}}$ sitting at the start of $I_{\beta}$ ). But, if $\beta+1<\operatorname{lh}(\mathcal{T})$, the copy of $E_{\beta}^{\mathcal{T}}$ (in $\mathcal{X}$ ) will be $E_{\delta_{\beta}}^{\mathcal{X}}$, not $E_{\gamma_{\beta}}^{\mathcal{X}}$ (unless $\left.\delta_{\beta}=\gamma_{\beta}\right)$. Here $\left(\gamma_{\beta}, \delta_{\beta}\right]_{\mathcal{X}}$ might

\footnotetext{
${ }^{20}$ Putative means that $M$ satisfies the first-order requirements of premousehood, but may be illfounded.
} 


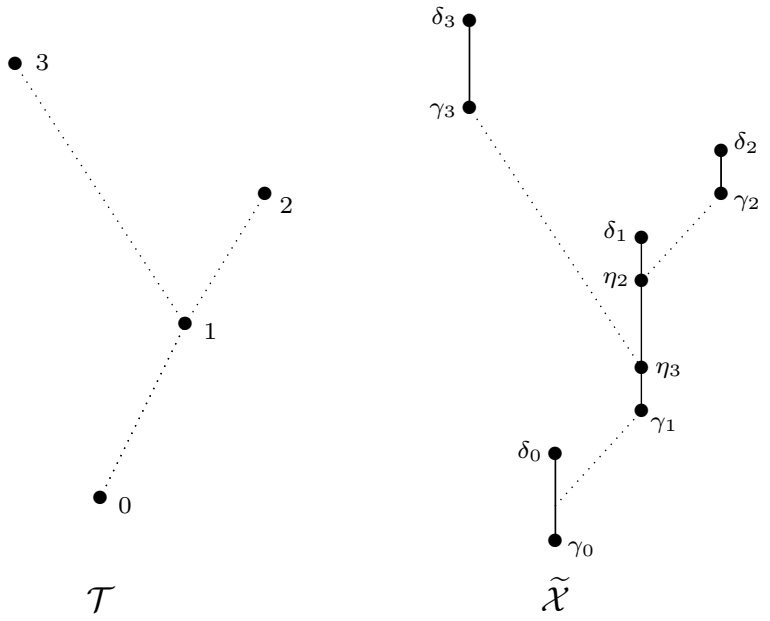

Figure 1: Preservation of tree structure, with $\operatorname{lh}(\mathcal{T})=\theta=4$. Bullets represent tree nodes. Dotted lines connect nodes with their predecessors; in particular, $\eta_{i}=\operatorname{pred}^{\mathcal{X}}\left(\gamma_{i}\right)$ for $i=2,3$. Solid lines represent $<^{\mathcal{X}}$-intervals. And $\widetilde{\mathcal{X}}$ is the restriction of $\mathcal{X}$ to $\bigcup_{i<4} I_{i}$.

actually drop in model, but it will not drop below the image of $E_{\beta}^{\mathcal{T}}$. However, if $\operatorname{lh}(\mathcal{T})=\beta+1$ then $\left(\gamma_{\beta}, \delta_{\beta}\right]_{\mathcal{X}}$ will not drop in model.

We will actually define a slightly more general notion: that of a tree embed$\operatorname{ding}(\mathcal{T}, \theta) \hookrightarrow \mathcal{X}$, where $\theta \leq \operatorname{lh}(\mathcal{T})$. If $\theta=\operatorname{lh}(\mathcal{T})$ or $\theta$ is a limit, this will be the same as a tree embedding $\mathcal{T}\lceil\theta \hookrightarrow \mathcal{X}$. But if $\theta=\beta+1<\operatorname{lh}(\mathcal{T})$, then we allow $\left(\gamma_{\beta}, \delta_{\beta}\right]_{\mathcal{X}}$ to drop in model, as long as it does not drop below the image of $E_{\beta}^{\mathcal{T}}$.

4.7 Definition (Tree embedding). Let $M$ be a $u$ - $k$-sound seg-pm, let $\mathcal{T}$ be a putative $\mathrm{u}$ - $k$-maximal tree on $M$, let $\mathcal{X}$ be a $\mathrm{u}$ - $k$-maximal tree on $M$, let $1 \leq \theta \leq \operatorname{lh}(\mathcal{T})$, and let $\Delta=\operatorname{ddd}^{(\mathcal{T}, \theta)}$.

A tree embedding $\Pi:(\mathcal{T}, \theta) \hookrightarrow \mathcal{X}$ from $(\mathcal{T}, \theta)$ to $\mathcal{X}$ is a system

$$
\Pi=\left(\mathcal{T},\left\langle I_{\beta}\right\rangle_{\beta<\theta} ;\left\langle I_{\beta i}, P_{\beta i}, \pi_{\beta i}\right\rangle_{(\beta, i) \in \Delta}\right)
$$

with properties $\mathrm{T} 1-\mathrm{T} 6$ below. We will see later that $\Pi$ is determined by $\left(\mathcal{T}, \mathcal{X},\left\langle I_{\beta}\right\rangle_{\beta<\theta}\right)$. While stating T1-T6, we also define various other uniquely determined objects. We sometimes denote $(\beta, i)$ with a single variable $x$. For $x=(\beta, i) \in \Delta$ let $m_{\beta}=m_{\beta}^{\mathcal{T}}$ and $M_{\beta i}=M_{x}=M_{x}^{\mathcal{T}}$.

T1. (Preservation of tree structure) See figure 1.

We have $I_{\beta} \in \operatorname{clint}^{\mathcal{X}}$ for each $\beta<\theta$. Let

$$
\left[\gamma_{\beta}, \delta_{\beta}\right]_{\mathcal{X}}=_{\mathrm{def}} I_{\beta} .
$$

Let $\Gamma: \theta \rightarrow \operatorname{lh}(\mathcal{X})$ be $\Gamma(\beta)=\gamma_{\beta}$. Then: 
(a) $\gamma_{0}=0$,

(b) $\Gamma$ preserves $<$, is continuous, sends successors (i.e. successor ordinals) to successors,

(c) $\beta_{0}<\mathcal{T} \beta_{1} \Longleftrightarrow \gamma_{\beta_{0}}<^{\mathcal{X}} \gamma_{\beta_{1}}$.

(d) $\mathrm{u}-\operatorname{deg}^{\mathcal{X}}\left(\gamma_{\beta}\right)=\mathrm{u}-\operatorname{deg}^{\mathcal{T}}(\beta)$.

(e) For $\beta+1<\theta$, we have $\gamma_{\beta+1}=\delta_{\beta}+1$.

(f) For $\beta+1<\theta$, letting $\xi=\operatorname{pred}^{\mathcal{T}}(\beta+1)$, we have

$$
\operatorname{pred}^{\mathcal{X}}\left(\gamma_{\beta+1}\right) \in I_{\xi}
$$

and

$$
\mathscr{D}^{\mathcal{X}} \cap\left(\gamma_{\xi}, \gamma_{\beta+1}\right]_{\mathcal{X}}=\emptyset \Longleftrightarrow \beta+1 \notin \mathscr{D}^{\mathcal{T}} .
$$

(So (i) the $<$-intervals ${ }^{21}\left[\gamma_{\beta}, \delta_{\beta}\right]$ partition $\sup _{\beta<\theta} \delta_{\beta}$, (ii) for $\xi, \zeta<\theta$,

$$
\left(\gamma_{\xi}, \gamma_{\zeta}\right]_{\mathcal{X}} \cap \mathscr{D}^{\mathcal{X}}=\emptyset \Longleftrightarrow(\xi, \zeta]_{\mathcal{T}} \cap \mathscr{D}^{\mathcal{T}}=\emptyset
$$

and (iii) for each limit $\beta<\theta$, we have $\Gamma^{\prime \prime}[0, \beta)_{\mathcal{T}} \subseteq_{\text {cof }}\left[0, \gamma_{\beta}\right)_{\mathcal{X}}$.)

T2. (Structure of $\left.I_{\beta}\right)$ Let $(\beta, i) \in \Delta$. Then:

(a) $I_{\beta i} \in \operatorname{clint}^{\mathcal{X}}$ and $I_{\beta i} \subseteq I_{\beta}$. Let $\left[\gamma_{\beta i}, \delta_{\beta i}\right]_{\mathcal{X}}={ }_{\operatorname{def}} I_{\beta i}$.

(b) $\gamma_{\beta 0}=\gamma_{\beta}$ and $\delta_{\beta m_{\beta}}=\delta_{\beta}$.

(c) If $(\beta, i+1) \in \Delta$ then $\gamma_{\beta, i+1}=\delta_{\beta i}$.

(Therefore, $I_{\beta 0}, \ldots, I_{\beta m_{\beta}}$ essentially partition $I_{\beta}$ into an increasing sequence of closed $<^{\mathcal{X}}$-intervals; they just overlap at their endpoints.)

(d) If $\gamma_{\beta i}<\delta_{\beta i}$ then let $\varepsilon_{\beta i}=\min \left(I_{\beta i} \backslash\left\{\gamma_{\beta i}\right\}\right)$.

(e) If $\gamma_{\beta 0}<\delta_{\beta 0}$ then $\left(\gamma_{\beta 0}, \delta_{\beta 0}\right]_{\mathcal{X}}$ does not drop in model (but may drop in degree).

(f) If $i>0$ and $\gamma_{\beta i}<\delta_{\beta i}$ then $\mathscr{D}^{\mathcal{X}} \cap\left(\gamma_{\beta i}, \delta_{\beta i}\right]_{\mathcal{X}}=\left\{\varepsilon_{\beta i}\right\}$.

T3. (Model embeddings) See figure 2. Let $x=(\beta, i) \in \Delta$. Then:

(a) $P_{\beta i}$ is a segmented-premouse and $\pi_{\beta i}: M_{\beta i} \rightarrow P_{\beta i}$ is an embedding. Let $P_{\beta}=P_{\beta 0}$ and $\pi_{\beta}=\pi_{\beta 0}$ (but maybe $I_{\beta} \neq I_{\beta 0}$ ).

(b) $P_{0}=M$ and $\pi_{0}=\mathrm{id}: M \rightarrow M$.

(c) $P_{\beta}=M_{\gamma_{\beta}}^{\mathcal{X}}\left(\right.$ recall $\left.\gamma_{\beta}=\gamma_{\beta 0}\right)$.

(d) $\pi_{\beta}$ is a near $\mathrm{u}-\operatorname{deg}^{\mathcal{T}}(\beta)$-embedding.

(e) Suppose $i>0$. Then $P_{x} \triangleleft M_{\gamma_{x}}^{\mathcal{X}}$ and $\pi_{x}$ is fully elementary. If $\gamma_{x}<\delta_{x}$ then $P_{x}=M_{\varepsilon_{x}}^{* \mathcal{X}}$.

\footnotetext{
${ }^{21}$ Note this is $<$, not $<{ }^{\mathcal{T}}$.
} 


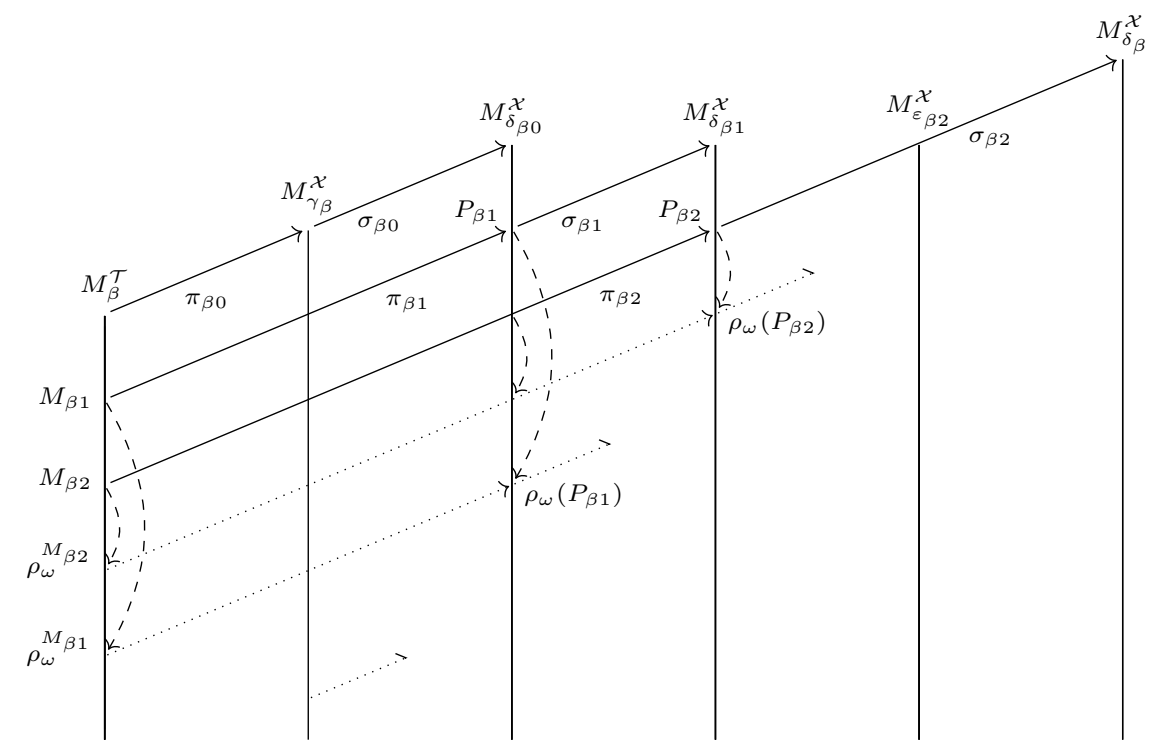

Figure 2: Model embeddings, with $m_{\beta}=2$. Vertical lines represent models, with length roughly corresponding to ordinal height. Solid arrows represent embeddings $\pi_{\beta i}$ and $\sigma_{\beta i}$, with $\operatorname{cr}\left(\sigma_{\beta i}\right)$ roughly at the origin of a short dotted half-headed arrow. Dotted full-headed arrows indicate certain threads under embeddings. Dashed curved arrows point to the $\omega^{\text {th }}$ projectum of the structure at their origin. Note that $M_{\delta_{\beta i}}^{\mathcal{X}}=M_{\gamma_{\beta, i+1}}^{\mathcal{X}}$ and for $i=0,1$. 


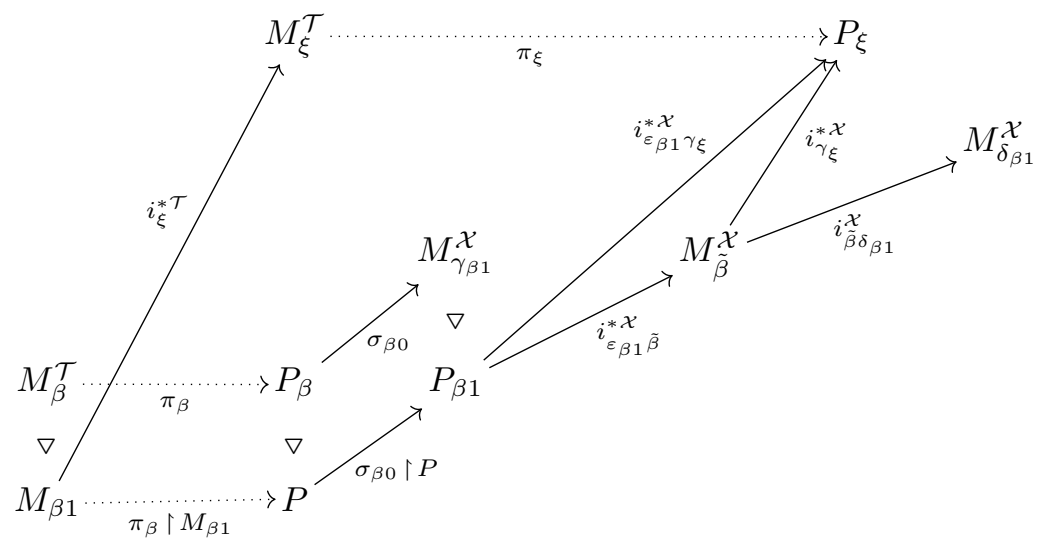

Figure 3: Embedding commutativity part T5(b), with $i=1$ and $\gamma_{\beta 1}<\widetilde{\beta}<$ $\delta_{\beta 1}$. The diagram commutes. Solid arrows are iteration embeddings and their restrictions; dotted arrows are copy embeddings and their restrictions. In the figure, $P=\pi_{\beta}\left(M_{\beta 1}\right)$.

(f) If $\gamma_{x}<\delta_{x}$ let

$$
\sigma_{\beta i}=\sigma_{x}=i_{\varepsilon_{x}, \delta_{x}}^{* \mathcal{X}}: P_{x} \rightarrow M_{\delta_{x}}^{\mathcal{X}}
$$

otherwise let $\sigma_{x}: P_{x} \rightarrow P_{x}$ be the identity. Let $\tau_{x}=\sigma_{x} \circ \pi_{x}$.

(g) Suppose $(\beta, i+1) \in \Delta$. Then $P_{\beta, i+1}=\tau_{\beta i}\left(M_{\beta, i+1}\right)$ and $\pi_{\beta, i+1}=$ $\tau_{\beta i} \uparrow M_{\beta, i+1}$.

T4. (Extender copying) For $\beta+1 \leq \theta$, let $\omega_{\beta}=\tau_{\beta m_{\beta}}$ and let $Q_{\beta}$ be the codomain of $\omega_{\beta}$; that is,

- if $\gamma_{\beta m_{\beta}}=\delta_{\beta m_{\beta}}$ then $Q_{\beta}=P_{\beta m_{\beta}}$, and

- if $\gamma_{\beta m_{\beta}}<\delta_{\beta m_{\beta}}$ then $Q_{\beta}=M_{\delta_{\beta}}^{\mathcal{X}}$.

If $\beta+1<\theta$ then $E_{\delta_{\beta}}^{\mathcal{X}}=F^{Q_{\beta}}$ (so $E_{\delta_{\beta}}^{\mathcal{X}}$ is the copy of $E_{\beta}^{\mathcal{T}}$ under $\omega_{\beta}$ ).

T5. (Embedding commutativity) Let $(\beta, i),(\alpha+1,0),(\xi, 0) \in \Delta$ be such that $\beta<<^{\mathcal{T}} \alpha+1 \leq \leq^{\mathcal{T}} \xi$ and $\beta=\operatorname{pred}^{\mathcal{T}}(\alpha+1)$ and $M_{\beta i}=M_{\alpha+1}^{* \mathcal{T}}$. Then:

(a) If $(\beta, \xi]_{\mathcal{T}} \cap \mathscr{D}^{\mathcal{T}}=\emptyset$ (so $i=0$ and $\left.\left(\gamma_{\beta}, \gamma_{\xi}\right]_{\mathcal{X}} \cap \mathscr{D}^{\mathcal{X}}=\emptyset\right)$ then

$$
\pi_{\xi} \circ i_{\beta, \xi}^{\mathcal{T}}=i_{\gamma_{\beta}, \gamma_{\xi}}^{\mathcal{X}} \circ \pi_{\beta}
$$

and $\operatorname{pred}^{\mathcal{X}}\left(\gamma_{\alpha+1}\right) \in I_{\beta 0}$.

(b) See figure 3. Suppose $\xi=\alpha+1 \in \mathscr{D}^{\mathcal{T}}$ (so $i>0$ ). Let $\widetilde{\beta}=\operatorname{pred}^{\mathcal{X}}\left(\gamma_{\xi}\right)$. Then $\widetilde{\beta} \in I_{\beta i}$ and:

- If $\widetilde{\beta}=\gamma_{\beta i}$ then $\gamma_{\xi} \in \mathscr{D}^{\mathcal{X}}$ and $M_{\gamma_{\xi}}^{* \mathcal{X}}=P_{\beta i}$ and $\pi_{\xi} \circ i_{\xi}^{* \mathcal{T}}=i_{\gamma_{\xi}}^{* \mathcal{X}} \circ \pi_{\beta i}$; 
- If $\widetilde{\beta}>\gamma_{\beta i}$ then $\gamma_{\xi} \notin \mathscr{D}^{\mathcal{X}}$ and $\pi_{\xi} \circ i_{\xi}^{* \mathcal{T}}=i_{\varepsilon_{x}, \gamma_{\xi}}^{* \mathcal{X}} \circ \pi_{\beta i}$.

T6. (Embedding agreement) For $\beta+1<\theta$ and $\left(\beta^{\prime}, i^{\prime}\right) \in \Delta$ with $\beta<\beta^{\prime}$ :

$$
\begin{aligned}
- & \omega_{\beta}\left\lceil\iota_{\beta}^{\mathcal{T}} \subseteq \pi_{\beta^{\prime} i^{\prime}}\right. \\
- & \omega_{\beta}(\alpha) \leq \pi_{\beta^{\prime} i^{\prime}}(\alpha) \text { for all } \alpha<\operatorname{ind}\left(E_{\beta}^{\mathcal{T}}\right), \\
- & \text { if } \operatorname{ind}\left(E_{\beta}^{\mathcal{T}}\right)<\operatorname{OR}\left(M_{\beta^{\prime} i^{\prime}}\right) \text { then } \operatorname{ind}\left(E_{\delta_{\beta}}^{\mathcal{X}}\right) \leq \pi_{\beta^{\prime} i^{\prime}}\left(\operatorname{ind}\left(E_{\beta}^{\mathcal{T}}\right)\right), \\
- & \text { if } \operatorname{ind}\left(E_{\beta}^{\mathcal{T}}\right)=\operatorname{OR}\left(M_{\beta^{\prime} i^{\prime}}\right) \text { then } \beta^{\prime}=\beta+1, i^{\prime}=0, \operatorname{ind}\left(E_{\delta_{\beta}}^{\mathcal{X}}\right)= \\
& \operatorname{OR}\left(M_{\gamma_{\beta+1}}^{\mathcal{X}}\right), \pi_{\beta+1}=\omega_{\beta}, M_{\gamma_{\beta+1}}^{* \mathcal{X}}=Q_{\alpha} \text { where } \alpha=\operatorname{pred}^{\mathcal{T}}(\beta+1) .{ }^{22} \dashv
\end{aligned}
$$

The analogue for wcpms is much simpler, as there is no dropping to consider:

4.8 Definition (Tree embedding for wcpms). Let $M$ be a wcpm, let $\mathcal{T}$ be a putative normal tree on $M$, let $\mathcal{X}$ be a normal tree on $M$, and let $1 \leq \theta \leq \operatorname{lh}(\mathcal{T})$. A tree embedding $\Pi:(\mathcal{T}, \theta) \hookrightarrow \mathcal{X}$ from $(\mathcal{T}, \theta)$ to $\mathcal{X}$ is a system $\Pi$ of form $\Pi=\left(\mathcal{T},\left\langle I_{\beta}, \pi_{\beta}\right\rangle_{\beta<\theta}\right)$ satisfying conditions $\mathrm{T}_{\mathrm{c}} 1-\mathrm{T}_{\mathrm{c}} 6$ below. $^{23}$

$\mathrm{T}_{\mathrm{c}}$ 1. (Preservation of tree structure) Exactly the assertion of condition 4.7(T1), minus the references to dropping and degrees.

$\mathrm{T}_{\mathrm{c}} 3$. (Model embeddings) See figure 2. For all $\beta<\theta$ :

(a) Let $P_{\beta}=M_{\gamma_{\beta}}^{\mathcal{X}}$.

(b) $\pi_{\beta}: M_{\beta} \rightarrow P_{\beta}$ is a coarse 0 -embedding.

(c) $\pi_{0}=$ id $: M \rightarrow M$.

(d) Let $\sigma_{\beta}=i_{\gamma_{\beta} \delta_{\beta}}^{\mathcal{X}}: P_{\beta} \rightarrow M_{\delta_{\beta}}^{\mathcal{X}}$ and $\tau_{\beta}=\sigma_{\beta} \circ \pi_{\beta}$.

T 4 . (Extender copying) For $\beta+1<\theta$, we have $E_{\delta_{\beta}}^{\mathcal{X}}=\tau_{\beta}\left(E_{\beta}^{\mathcal{T}}\right) .{ }^{24}$

$\mathrm{T}_{\mathrm{c}}$ 5. (Embedding commutativity) If $\beta<\mathcal{T} \xi<\theta$ and $\alpha+1=\operatorname{succ}^{\mathcal{T}}(\beta, \xi)$ then

$$
\pi_{\xi} \circ i_{\beta \xi}^{\mathcal{T}}=i_{\gamma_{\beta} \gamma_{\xi}}^{\mathcal{X}} \circ \pi_{\beta}
$$

$\mathrm{T}_{\mathrm{c}} 6$. (Embedding agreement) Let $\beta+1 \leq \beta^{\prime}<\theta$ and $\varrho=\varrho_{\beta}^{\mathcal{T}}$. Then

$$
\tau_{\beta}\left\lceil V_{\varrho}^{M_{\beta}^{\mathcal{T}}} \subseteq \pi_{\beta^{\prime}} \text { and } \varrho_{\delta_{\beta}}^{\mathcal{X}}=\tau_{\beta}(\varrho) \leq \pi_{\beta^{\prime}}(\varrho) .\right.
$$

4.9 Definition. A tree embedding $\Pi:(\mathcal{T}, \theta) \hookrightarrow \mathcal{X}$ has u-degree $k$ iff $\mathcal{T}, \mathcal{X}$ are $\mathrm{u}$ - $k$-maximal. (There is a unique such $k$, since $k=\mathrm{u}$ - $\operatorname{deg}^{\mathcal{T}}(0)$.)

\footnotetext{
${ }^{22}$ It follows that we are using MS-indexing, $E_{\beta}^{\mathcal{T}}$ is superstrong and $M_{\beta+1}^{\mathcal{T}}$ is active type 2, $E_{\delta_{\beta}}^{\mathcal{X}}$ is superstrong and $M_{\delta_{\beta}+1}^{\mathcal{X}}$ is active type 2 .

${ }^{23}$ There is no analogue of condition $4.7(\mathrm{~T} 2)$, because there is no dropping or degrees.

${ }^{24}$ Note that because there is no dropping, we do not define $\omega_{\beta}$ and $Q_{\beta}$ here. The map $\tau_{\beta}$ lifts $E_{\beta}^{\mathcal{T}}$ to $E_{\delta_{\beta}}^{\mathcal{X}}$ here.
} 
4.10 Definition. A tree embedding $\Pi: \mathcal{T} \hookrightarrow \mathcal{X}$ from $\mathcal{T}$ to $\mathcal{X}$ is a tree embedding $\Pi:(\mathcal{T}, \ln (\mathcal{T})) \hookrightarrow \mathcal{X}$.

Clearly if $\Pi: \mathcal{T} \hookrightarrow \mathcal{X}$ then $\mathcal{T}$ is in fact an iteration tree (it has well-defined and wellfounded models). We record some notation:

4.11 Definition. Let $\Pi$ be a tree embedding. Fix notation as in 4.7. Define $Q_{\beta i}=\operatorname{cod}\left(\tau_{\beta i}\right)$. That is, $Q_{\beta i}=P_{\beta i}$ if $\gamma_{\beta i}=\delta_{\beta i}$, and $Q_{\beta i}=M_{\delta_{\beta i}}^{\mathcal{X}}$ otherwise. Let $i_{\beta}$ be the least $i$ such that $\delta_{\beta}=\delta_{\beta i}$. So $Q_{\beta}=Q_{\beta m_{\beta}} \unlhd Q_{\beta i_{\beta}}=M_{\delta_{\beta}}^{\mathcal{X}}$.

We use the subscript ${ }^{25}$ " $\Pi$ " to indicate the objects associated to $\Pi$. That is, $I_{\Pi \beta}=I_{\beta}$ for $\beta<\theta$, and $\Gamma_{\Pi}=\Gamma$, and likewise for $\gamma_{\beta}, \delta_{\beta}, P_{\beta}, \pi_{\beta}, Q_{\beta}, \omega_{\beta}, i_{\beta}$ for $\beta<\theta$, and $I_{\beta i}, P_{\beta i}, \pi_{\beta i}, \gamma_{\beta i}, \delta_{\beta i}, \sigma_{\beta i}, \tau_{\beta i}, Q_{\beta i}$ for $(\beta, i) \in \Delta$.

4.12 Definition $\left(j_{\xi \eta}^{\mathcal{X}}\right)$. Let $\Pi:(\mathcal{T}, \theta) \hookrightarrow \mathcal{X}$ be a tree embedding and $\gamma_{\beta}=\gamma_{\Pi \beta}$, etc. Let $\beta<\theta$. Let $\xi, \eta \in I_{\beta}$ with $\xi \leq \eta$. Then $j_{\xi \eta}^{\mathcal{X}}$ denotes the embedding with domain as large as possible, given by composing iteration embeddings $i_{\mu \nu}^{\mathcal{X}}$ and $i_{\mu \nu}^{* \mathcal{X}}$ with $\xi \leq^{\mathcal{X}} \mu \leq^{\mathcal{X}} \nu \leq^{\mathcal{X}} \eta$. That is, let $m, n$ be least such that $\xi \in I_{\beta m}$ and $\eta \in I_{\beta n}$ respectively. If $m=n$ then $j_{\xi \eta}^{\mathcal{X}}=\operatorname{def} i_{\xi \eta}^{\mathcal{X}}$. If $m<n$ then letting $\varepsilon=\varepsilon_{\beta n}$ and $\delta=\delta_{\beta, n-1}=\gamma_{\beta n}$,

$$
j_{\xi \eta}^{\mathcal{X}}={ }_{\operatorname{def}} i_{\varepsilon \eta}^{* \mathcal{X}} \circ j_{\xi \delta}^{\mathcal{X}},
$$

where $\operatorname{dom}\left(j_{\xi \eta}^{\mathcal{X}}\right)=M_{\xi}^{\mathcal{X}}$ if $m=n$, and $\operatorname{dom}\left(j_{\xi \eta}^{\mathcal{X}}\right)=j_{\gamma_{\beta} \xi}^{\mathcal{X}}\left(\pi_{\beta 0}\left(M_{\beta n}\right)\right)$ if $m<n . \quad \dashv$

4.13 Definition $\left(\pi_{\beta \kappa}: M_{\beta \kappa} \rightarrow P_{\beta \kappa}\right.$ and $\left.n_{\beta \kappa}\right)$. (Figure 4.) Let $\Pi:(\mathcal{T}, \theta) \hookrightarrow \mathcal{X}$ be a tree embedding and $\gamma_{\beta}=\gamma_{\Pi \beta}$, etc. Let $\beta<\theta$. Let $\kappa \in\left[\omega, \operatorname{OR}\left(M_{\beta}^{\mathcal{T}}\right)\right)$, with $\kappa<\widetilde{\nu}_{\beta}^{\mathcal{T}}$ if $\beta+1<\operatorname{lh}(\mathcal{T})$, and $\kappa \leq \operatorname{OR}\left(M_{\beta}^{\mathcal{T}}\right)$ if $\beta+1=\operatorname{lh}(\mathcal{T})$. We will define $i_{\beta \kappa}, n_{\beta \kappa}, M_{\beta \kappa}, \gamma_{\beta \kappa}, P_{\beta \kappa}$ and $\pi_{\beta \kappa}: M_{\beta \kappa} \rightarrow P_{\beta \kappa}$.

If $\beta+1=\operatorname{lh}(\mathcal{T})$ and $\kappa=\operatorname{OR}\left(M_{\beta}^{\mathcal{T}}\right)$ then let $i_{\beta \kappa}=n_{\beta \kappa}=0, M_{\beta \kappa}=M_{\beta}^{\mathcal{T}}$, $P_{\beta \kappa}=Q_{\beta}, \gamma_{\beta \kappa}=\delta_{\beta}$ and $\pi_{\beta \kappa}=\omega_{\beta}$.

Now suppose either $\beta+1<\ln (\mathcal{T})$ or $\kappa<\operatorname{OR}\left(M_{\beta}^{\mathcal{T}}\right)$. Let $i_{\beta \kappa}$ be the largest $i<\omega$ such that either $i=0$ or $\rho_{\omega}\left(M_{\beta i}\right) \leq \kappa$. Let $i=i_{\beta \kappa}$. Set $M_{\beta \kappa}={ }_{\operatorname{def}} M_{\beta i}$. Let $n_{\beta \kappa}$ be the largest $n<\omega$ such that

$$
\left(M_{\beta \kappa}, n\right) \unlhd\left(M_{\beta 0}, \mathrm{u}-\operatorname{deg}^{\mathcal{T}}(\beta)\right)
$$

and $\kappa<\mathrm{u}-\rho_{n}^{M_{\beta \kappa}}$.

Let $\gamma_{\beta \kappa}$ be the least $\gamma \in I_{\beta i}$ such that either $\gamma=\delta_{\beta i}$ or

$$
\operatorname{cr}\left(j_{\gamma \delta_{\beta i}}^{\mathcal{X}}\right)>j_{\gamma_{\beta i} \gamma}^{\mathcal{X}} \circ \pi_{\beta i}(\kappa) .
$$

Let $\gamma=\gamma_{\beta \kappa}$. If $\gamma=\gamma_{\beta i}$ then $P_{\beta \kappa}={ }_{\text {def }} P_{\beta i}$ and $\pi_{\beta \kappa}={ }_{\text {def }} \pi_{\beta i}$. If $\gamma>\gamma_{\beta i}$ then $P_{\beta \kappa}={ }_{\text {def }} M_{\gamma}^{\mathcal{X}}$ and $\pi_{\beta \kappa}={ }_{\text {def }} j_{\gamma_{\beta i} \gamma}^{\mathcal{X}} \circ \pi_{\beta i}$.

We write $\pi_{\Pi \beta \kappa}=\pi_{\beta \kappa}$, etc.

4.14 Lemma. Let $\Pi:(\mathcal{T}, \theta) \hookrightarrow \mathcal{X}$ be a tree embedding. Let $\alpha \in I_{\Pi \xi}$ and $\delta \leq^{\mathcal{X}} \alpha$. Then $\delta \in I_{\Pi \zeta}$ for some $\zeta \leq^{\mathcal{T}} \xi$.

\footnotetext{
${ }^{25}$ The superscript position of this notation will be used for another purpose.
} 


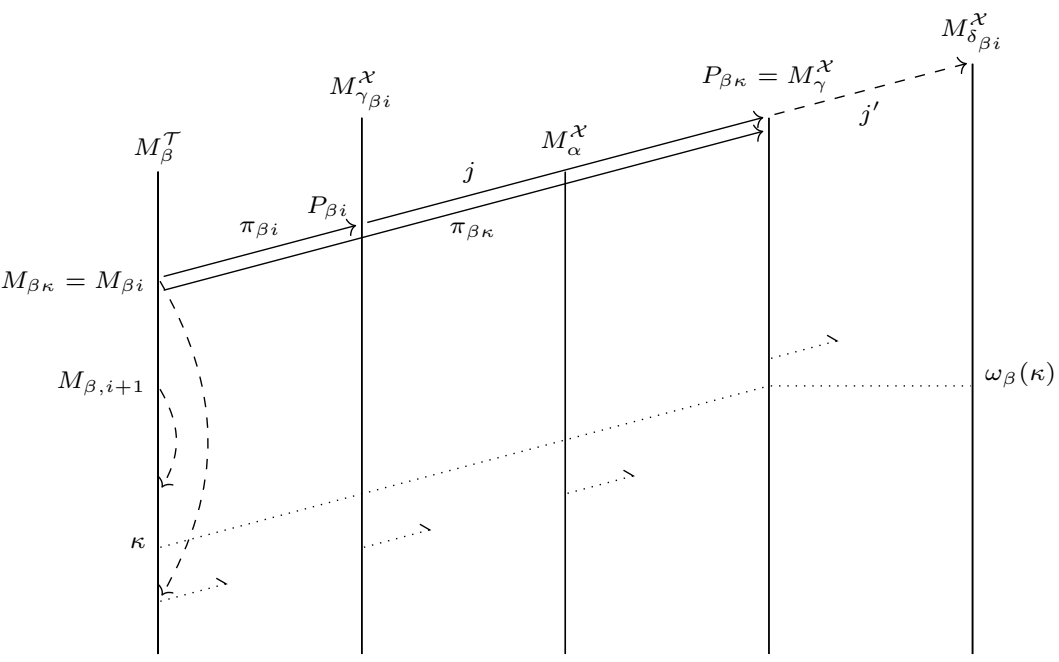

Figure 4: A typical picture for the embedding $\pi_{\beta \kappa}: M_{\beta \kappa} \rightarrow P_{\beta \kappa}$, when $i=i_{\beta \kappa}$ and $\gamma_{\beta i}<\gamma=\gamma_{\beta \kappa}<\delta_{\beta i}$. Note $\pi_{\beta \kappa}=j \circ \pi_{\beta i}$, where $j=j_{\gamma_{\beta i}, \gamma_{\beta \kappa}}^{\mathcal{X}}$. The long dotted path indicates the trajectory of $\kappa$. Critical points are indicated by dotted half-headed arrows. (Where critical points are shown strictly below the image of $\kappa$ in the figure, they could in general equal that image.) Also, $\alpha \in\left(\gamma_{\beta i}, \gamma_{\beta \kappa}\right)_{\mathcal{X}}$ and $j^{\prime}=i_{\gamma_{\beta \kappa}, \delta_{\beta i}}^{\mathcal{X}}$. 
The reader will easily verify the lemma above (proceed by induction). Part 3 of the next lemma ensures that when we want to extend tree embeddings, we will not encounter any difficulties regarding condition $\mathrm{T} 1(\mathrm{~d})$.

4.15 Lemma. Let $\Pi:(\mathcal{T}, \theta) \hookrightarrow \mathcal{X}$ be a tree embedding and let $\gamma_{\beta}=\gamma_{\Pi \beta}$, etc. Let $\pi=\pi_{\beta \kappa}$ and $n=n_{\beta \kappa}$ and $\gamma=\gamma_{\beta \kappa}$. Then

1. $\pi$ is a near $\mathrm{u}-n$-embedding,

2. $\left(P_{\beta \kappa}, n\right) \unlhd^{\mathrm{u}}\left(M_{\gamma}^{\mathcal{X}}, \mathrm{u}-\operatorname{deg}^{\mathcal{X}}(\gamma)\right)$ and

3. if $\left(P_{\beta \kappa}, n\right) \triangleleft^{\mathrm{u}}\left(M_{\gamma}^{\mathcal{X}}, \mathrm{u}-\operatorname{deg}^{\mathcal{X}}(\gamma)\right)$ and $\kappa<\mathrm{OR}\left(M_{\beta \kappa}\right)$ then $\pi_{\beta \kappa}(\kappa) \geq \mathrm{u}-\rho_{n+1}\left(P_{\beta \kappa}\right)$.

Proof Sketch. Part 3: Let $i=i_{\beta \kappa}$. If $i=0$, use that $\pi_{\beta}$ is a near u- $(n+1)$ embedding (see condition T3(d)), as are the relevant iteration maps; in the other case it is similar, but $\pi_{\beta i}$ is fully elementary.

4.16 Definition. Let $\Pi:(\mathcal{T}, \theta) \hookrightarrow \mathcal{X}$. Let $\beta \in \theta \cap \operatorname{lh}(\mathcal{T})^{-}$and $\gamma \in I_{\Pi \beta}$. Then $E_{\gamma}^{\Pi}$ denotes the copy $F$ of $E_{\beta}^{\mathcal{T}}$ in $\mathbb{E}_{+}\left(M_{\gamma}^{\mathcal{X}}\right)$. (That is, letting $k=j_{\gamma_{\beta} \gamma}^{\mathcal{X}} \circ \pi_{\beta}$, if $E_{\beta}^{\mathcal{T}} \in \operatorname{dom}(k)$ then $F=k\left(E_{\beta}^{\mathcal{T}}\right)$, and otherwise $\left.F=F\left(M_{\gamma}^{\mathcal{X}}\right)\right)$. We say that $\Pi$ is bounding iff $\operatorname{ind}\left(E_{\gamma}^{\Pi}\right) \geq \operatorname{ind}\left(E_{\gamma}^{\mathcal{X}}\right)$ for all $\operatorname{such} \beta, \gamma$.

We will only really be interested in bounding tree embeddings, and in this case we have the following easy observation:

4.17 Lemma. Let $\Pi:(\mathcal{T}, \theta) \hookrightarrow \mathcal{X}$ be bounding. Suppose $\theta=\beta+1<\operatorname{lh}(\mathcal{T})$. Then $E_{\delta_{\Pi \beta}}^{\Pi}$ is $\mathcal{X} \uparrow\left(\delta_{\Pi \beta}+1\right)$-normal.

We now consider the existence and uniqueness of tree embeddings.

4.18 Definition. Let $\mathcal{T}, \mathcal{X}$ be putative u- $k$-maximal trees on $M$, with $\mathcal{X}$ an iteration tree. The trivial tree embedding $\Pi:(\mathcal{T}, 1) \hookrightarrow \mathcal{X}$ is the unique one such that $I_{\Pi 0}=[0,0]$; that is,

$$
\Pi=\left(\mathcal{T},\langle[0,0]\rangle ;\left\langle I_{0 i}, P_{0 i}, \pi_{0 i}\right\rangle_{(0, i) \in \Delta}\right)
$$

where $\Delta=\operatorname{ddd}^{(\mathcal{T}, 1)}$ and $I_{0 i}=[0,0]$ and $P_{0 i}=M_{0 i}^{\mathcal{T}}$ and $\pi_{0 i}=\mathrm{id}$.

We will give two lemmas describing how we can propagate tree embeddings via ultrapowers. The first of these involves copying an extender. Part of this is a natural variant of the fact that the copying construction propagates near embeddings (see [9]), and to state this we need the following definition:

4.19 Definition. Let $\mathcal{T}, \mathcal{X}, \theta$ be as in 4.7. A $*$-tree embedding $\Pi$ from $(\mathcal{T}, \theta)$ to $\mathcal{X}$, denoted $\Pi:(\mathcal{T}, \theta) \hookrightarrow^{*} \mathcal{X}$, is a system as in 4.7 , but replacing $\mathrm{T} 3(\mathrm{~d})$ with the requirement that $\pi_{\beta}$ be $\mathrm{r} \Sigma_{n}$-elementary where $n=\mathrm{u}$ - $\operatorname{deg}^{\mathcal{T}}(\beta)$.

4.20 Lemma. Let $\Pi^{\prime}:(\mathcal{T}, \theta) \hookrightarrow \mathcal{X}^{\prime}$ have u-degree $k$. Let $\gamma_{\alpha}^{\prime}=\gamma_{\Pi^{\prime} \alpha}$, etc. Suppose that $\theta=\alpha+1<\ln (\mathcal{T})$ and $\ln \left(\mathcal{X}^{\prime}\right)=\delta_{\alpha}^{\prime}+1$ and $E_{\delta_{\alpha}^{\prime}}^{\Pi^{\prime}}$ is $\mathcal{X}$-normal. Suppose that the putative $\mathrm{u}$ - $k$-maximal tree $\mathcal{X}^{\prime \prime}={ }_{\operatorname{def}} \mathcal{X}^{\prime} \wedge\left\langle E_{\delta_{\alpha}^{\prime}}^{\Pi^{\prime}}\right\rangle$ has wellfounded last model.

Then (i) $M_{\alpha+1}^{\mathcal{T}}$ is wellfounded, (ii) there is a unique pair $(\mathcal{X}, \Pi)$ such that: 
- $\mathcal{X}$ is a u-k-maximal tree extending $\mathcal{X}^{\prime}$ with $\ln (\mathcal{X})=\operatorname{lh}\left(\mathcal{X}^{\prime}\right)+1$,

$-\Pi:(\mathcal{T}, \theta+1) \hookrightarrow^{*} \mathcal{X}$, and

$-\Pi^{\prime} \subseteq \Pi$,

(iii) $\mathcal{X}=\mathcal{X}^{\prime \prime},\left(\right.$ iv) $\Pi:(\mathcal{T}, \theta+1) \hookrightarrow \mathcal{X}$, (v) if $\theta+1<\operatorname{lh}(\mathcal{T})$ then $E_{\delta_{\alpha}^{\prime}+1}^{\Pi}$ is $\mathcal{X}$-normal, and (vi) if $\Pi^{\prime}$ is bounding then so is $\Pi$.

Before we prove the lemma, we state two easy consequences:

4.21 Corollary. Every $*$-tree embedding is a tree embedding.

4.22 Corollary. Let $\Pi:(\mathcal{T}, \theta) \hookrightarrow \mathcal{X}$ and $\Pi^{\prime}:(\mathcal{T}, \theta) \hookrightarrow \mathcal{X}$ be tree embeddings such that $\delta_{\beta}^{\Pi}=\delta_{\beta}^{\Pi^{\prime}}$ for all $\beta<\theta$. Then $\Pi=\Pi^{\prime}$.

Proof of 4.20. We first exhibit $(\mathcal{X}, \Pi)$ as in (ii); then (i) follows. We then prove the uniqueness of $(\mathcal{X}, \Pi)$, and then (iv), and leave the rest to the reader.

We use $\mathcal{X}=\mathcal{X}^{\prime \prime}$. In defining the components of $\Pi$, we will write $I_{\beta}=I_{\Pi \beta}$, etc. Most of $\Pi$ is already determined by the requirement that $\Pi^{\prime} \subseteq \Pi$, so we just define the rest. Let

$$
I_{\alpha+1}=I_{\alpha+1,0}=\left[\delta_{\alpha}^{\prime}+1, \delta_{\alpha}^{\prime}+1\right]
$$

and $P_{\alpha+1,0}=M_{\delta_{\alpha}^{\prime}+1}^{\mathcal{X}}$. It just remains to define $\pi_{\alpha+1,0}: M_{\alpha+1}^{\mathcal{T}} \rightarrow M_{\delta_{\alpha}+1}^{\mathcal{X}}$, and we claim that we can do this using the Shift Lemma.

For let $E=E_{\alpha}^{\mathcal{T}}$ and $\kappa=\operatorname{cr}(E)$ and $\beta=\operatorname{pred}^{\mathcal{T}}(\alpha+1)$ and $n=\mathrm{u}-\operatorname{deg}^{\mathcal{T}}(\alpha+1)$. Note $M_{\beta \kappa}^{\mathcal{T}}=M_{\alpha+1}^{* \mathcal{T}}$ and $n_{\beta \kappa}=n$ and $P_{\beta \kappa}=M_{\delta_{\alpha}+1}^{* \mathcal{X}}$ and

$$
\begin{aligned}
& \mathcal{P}(\kappa) \cap M_{\beta \kappa}^{\mathcal{T}}=\mathcal{P}(\kappa) \cap M_{\alpha}^{\mathcal{T}} \operatorname{lind}(E), \\
& \pi_{\beta \kappa} \backslash \mathcal{P}(\kappa)=\omega_{\beta} \backslash \mathcal{P}(\kappa)=\omega_{\alpha} \backslash \mathcal{P}(\kappa)
\end{aligned}
$$

and by $4.15, n=\mathrm{u}-\operatorname{deg}^{\mathcal{X}}\left(\delta_{\alpha}+1\right)$.

So we apply the (proof of the) Shift Lemma to $\pi_{\beta \kappa}$ and $\omega_{\alpha}$, defining $\pi_{\alpha+1,0}$, a weak $\mathrm{u}-n$-embedding. The embedding commutativity and agreement conditions are satisfied. So $M_{\alpha+1}^{\mathcal{T}}$ is wellfounded and $\Pi:(\mathcal{T}, \theta) \hookrightarrow^{*} \mathcal{X}$ and $\Pi^{\prime} \subseteq \Pi$.

The definitions we made were in fact the only ones possible; in the case of $\pi_{\alpha+1}$, this is because if $\pi: M_{\alpha+1}^{\mathcal{T}} \rightarrow M_{\delta_{\alpha}+1}^{\mathcal{X}}$ is $\mathrm{r} \Sigma_{n}$-elementary and satisfies the commutativity and agreement conditions, then $\pi$ is just as defined in the proof of the Shift Lemma. This gives uniqueness.

For (v), it remains to see that $\pi_{\alpha+1}$ is a near $\mathrm{u}-n$-embedding. This is proved almost as in [9]; we give a sketch so as to indicate the main difference.

For $\zeta+1<\operatorname{lh}(\mathcal{T})$, we say that strong closeness at $\zeta$ holds iff for each $a \in\left[\nu\left(E_{\zeta}^{\mathcal{T}}\right)\right]^{<\omega}$ there is a $\mathrm{u} \Sigma_{1}$ formula $\varphi_{a}$ and $q_{a} \in M_{\zeta+1}^{* \mathcal{T}}$ such that

$$
\left(E_{\zeta}^{\mathcal{T}}\right)_{a}=\left\{x \in M_{\zeta+1}^{* \mathcal{T}} \mid M_{\zeta+1}^{* \mathcal{T}} \models \varphi_{a}\left(q_{a}, x\right)\right\},
$$

and letting $\beta=\operatorname{pred}^{\mathcal{T}}(\zeta+1)$ and $\mu=\operatorname{cr}\left(E_{\zeta}^{\mathcal{T}}\right)$, so $M_{\gamma_{\zeta+1}^{* \mathcal{X}}}^{*}=P_{\beta \mu}$,

$$
\left(E_{\delta_{\zeta}}^{\mathcal{X}}\right)_{\omega_{\zeta}(a)}=\left\{x \in P_{\beta \mu} \mid P_{\beta \mu} \models \varphi_{a}\left(\pi_{\beta \mu}\left(q_{a}\right), x\right)\right\} .
$$


For $\varepsilon<\operatorname{lh}(\mathcal{T})$, we say translatability at $\varepsilon$ holds iff, letting $m=\mathrm{u}$ - $\operatorname{deg}^{\mathcal{T}}(\varepsilon)$, for all $(x, \varphi, \zeta+1)$ such that $x \in M_{\varepsilon}^{\mathcal{T}}$ and $\varphi$ is $\mathrm{u} \Sigma_{m+1}$ and $\zeta+1 \leq^{\mathcal{T}} \varepsilon$ and $(\zeta+1, \varepsilon]_{\mathcal{T}}$ does not drop in model or degree, there is $\left(x^{\prime}, \varphi^{\prime}\right)$ such that $x^{\prime} \in M_{\zeta+1}^{* \mathcal{T}}$ and $\varphi^{\prime}$ is $\mathrm{u} \Sigma_{m+1}$, and for all $\gamma<\mu==_{\text {def }} \operatorname{cr}\left(E_{\zeta}^{\mathcal{T}}\right)$, we have

$$
M_{\varepsilon}^{\mathcal{T}} \models \varphi(x, \gamma) \Longleftrightarrow M_{\zeta+1}^{* \mathcal{T}} \models \varphi^{\prime}\left(x^{\prime}, \gamma\right),
$$

and letting $\beta=\operatorname{pred}^{\mathcal{T}}(\zeta+1)$, for all $\gamma<\pi_{\beta \mu}(\mu)=\operatorname{cr}\left(E_{\delta_{\zeta}}^{\mathcal{X}}\right)$, we have

$$
M_{\gamma_{\varepsilon}}^{\mathcal{X}} \models \varphi\left(\pi_{\varepsilon}(x), \gamma\right) \Longleftrightarrow M_{\gamma_{\zeta+1}}^{* \mathcal{X}} \models \varphi^{\prime}\left(\pi_{\beta \mu}\left(x^{\prime}\right), \gamma\right) .
$$

(Recall that $\gamma_{\beta \mu}=\operatorname{pred}^{\mathcal{X}}\left(\gamma_{\zeta+1}\right)$ and $P_{\beta \mu}=M_{\gamma_{\zeta+1}}^{* \mathcal{X}}$.)

One proves strong closeness at $\zeta$ and translatability at $\varepsilon$, by simultaneous induction on $\max (\zeta+1, \varepsilon)$. This is basically as in [9], so the reader should refer there for the full argument, but there are a few extra details which arise here, which we explain. Fix $\zeta$ and consider the proof of strong closeness at $\zeta+1$. Let $\beta=\operatorname{pred}^{\mathcal{T}}(\zeta+1)$ and suppose $\beta<\zeta$. Let $E=E_{\zeta}^{\mathcal{T}}$ and $\kappa=\operatorname{cr}(E)$ and $F=E_{\delta_{\zeta}}^{\mathcal{X}}$. Suppose $\left(\kappa^{+}\right)^{\operatorname{ex}_{\beta}^{\mathcal{T}}}<\mathrm{OR}^{\operatorname{ex}_{\beta}^{\mathcal{T}}}$ but $E_{a} \notin \operatorname{ex}_{\beta}^{\mathcal{T}}$ for some $a \in\left[\nu_{E}\right]^{<\omega}$. Then as in [4, 6.1.5], $E=F\left(M_{\zeta}^{\mathcal{T}}\right)$ and $\beta<^{\mathcal{T}} \zeta$ and letting $\xi+1=\operatorname{succ}^{\mathcal{T}}(\beta, \zeta)$, we have

$-(\xi+1, \zeta]_{\mathcal{T}}$ does not drop, $\mathrm{u}-\operatorname{deg}^{\mathcal{T}}(\zeta)=\mathrm{u}-\operatorname{deg}^{\mathcal{T}}(\xi+1)=0$ and

$-\kappa<\mu=_{\operatorname{def}} \operatorname{cr}\left(i_{\xi+1, \zeta}^{* \mathcal{T}}\right)$.

Now $j={ }_{\text {def }} i_{\gamma_{\zeta} \delta_{\zeta}}^{\mathcal{X}}$ exists because $E=F\left(M_{\zeta}^{\mathcal{T}}\right)$, and note $\operatorname{cr}(F)<\operatorname{cr}\left(E_{\delta_{\xi}}^{\mathcal{X}}\right)<\operatorname{cr}(j)$ (where $\operatorname{cr}(j)=\infty$ if $j=$ id). So for $a \in\left[\nu_{E}\right]^{<\omega}$, letting $\bar{F}=F\left(M_{\gamma_{\zeta}}^{\mathcal{X}}\right)$, we have

$$
F_{\omega_{\zeta}(a)}=F_{j\left(\pi_{\zeta}(a)\right)}=\bar{F}_{\pi_{\zeta}(a)} .
$$

So using translatability at $\zeta$ as in [9], we get $(\varphi, q)$ such that $\varphi$ is $\mathrm{u} \Sigma_{1}$ and $q \in M_{\xi+1}^{* \mathcal{T}}=M_{\beta \mu}^{\mathcal{T}}$ and

$$
\begin{gathered}
\varphi(q, \cdot) \text { defines } E_{a} \text { over } M_{\xi+1}^{* \mathcal{T}}=M_{\beta \mu}^{\mathcal{T}}, \\
\varphi\left(\pi_{\beta \mu}(q), \cdot\right) \text { defines } F_{\omega_{\zeta}(a)} \text { over } M_{\gamma_{\xi+1}^{* \mathcal{X}}}^{* \mathcal{X}}=P_{\beta \mu} .
\end{gathered}
$$

So if $\gamma_{\beta \kappa}=\gamma_{\beta \mu}$ then we get strong closeness at $\zeta+1$ as in [9]. Suppose instead that $\gamma_{\beta \kappa}<\gamma_{\beta \mu}$. Let $k=j_{\gamma_{\beta \kappa} \gamma_{\beta \mu}}^{\mathcal{X}}$, so $\pi_{\beta \kappa}\left(M_{\beta \mu}^{\mathcal{T}}\right) \unlhd \operatorname{dom}(k)$,

$$
\begin{gathered}
\pi_{\beta \mu}=k \circ \pi_{\beta \kappa}\left\lceil M_{\beta \mu}^{\mathcal{T}},\right. \\
\operatorname{cr}(k)>\pi_{\beta \kappa}(\kappa)=\omega_{\beta}(\kappa)=\operatorname{cr}(F) .
\end{gathered}
$$

So if $M_{\beta \mu}^{\mathcal{T}}=M_{\beta \kappa}^{\mathcal{T}}$ then

$$
\varphi\left(\pi_{\beta \kappa}(q), \cdot\right) \text { defines } F_{\omega_{\zeta}(a)} \text { over } M_{\gamma_{\zeta+1}}^{* \mathcal{X}}=P_{\beta \kappa},
$$


as required. And if $M_{\beta \mu}^{\mathcal{T}} \triangleleft M_{\beta \kappa}^{\mathcal{T}}$ then we get a natural $\mathrm{u} \Sigma_{1}$ formula $\varphi^{\prime \prime}$ such that

$$
\begin{gathered}
\varphi^{\prime \prime}\left(\left(q, M_{\beta \mu}^{\mathcal{T}}\right), \cdot\right) \text { defines } E_{a} \text { over } M_{\beta \kappa}^{\mathcal{T}}, \\
\varphi^{\prime \prime}\left(\pi_{\beta \kappa}\left(q, M_{\beta \mu}^{\mathcal{T}}\right), \cdot\right) \text { defines } F_{\omega_{\zeta}(a)} \text { over } P_{\beta \kappa},
\end{gathered}
$$

again as required.

The second detail is as follows. Consider again strong closeness at $\zeta+1$. Let $\beta, \kappa, E, F$ be as before and suppose $\beta<\zeta$, but now with $\left(\kappa^{++}\right)^{\operatorname{ex}_{\beta}^{\mathcal{T}}}=\mathrm{OR}^{\operatorname{ex}_{\beta}^{\mathcal{T}}}$, and $E_{a} \in \mathrm{ex}_{\beta}^{\mathcal{T}}$ for every $a \in\left[\nu_{E}\right]^{<\omega}$. Then $\gamma_{\beta \kappa}=\delta_{\beta}$ and $\iota\left(\operatorname{ex}_{\beta}^{\mathcal{T}}\right)=\mathrm{OR}^{\mathrm{ex}_{\beta}^{\mathcal{T}}}$, so

$$
\pi_{\beta \kappa}\left\lceil\iota\left(\operatorname{ex}_{\beta}^{\mathcal{T}}\right)=\omega_{\beta}\left\lceil\mathrm{OR} \subseteq \omega_{\zeta},\right.\right.
$$

which implies that $\pi_{\beta \kappa}\left(E_{a}\right)=F_{\omega_{\zeta}(a)}$ for each $a$. This easily gives strong closeness in this case.

There are also similar considerations in other cases of strong closeness.

The proof of translatability at a successor $\varepsilon=\xi+1$ also involves an extra detail, with respect to $\zeta+1<^{\mathcal{T}} \xi+1$. Let $\delta=\operatorname{pred}^{\mathcal{T}}(\xi+1)$, so $\zeta+1 \leq{ }^{\mathcal{T}} \delta$ and $(\zeta+1, \delta]_{\mathcal{T}}$ does not drop in model or degree. Let $\kappa=\operatorname{cr}\left(E_{\zeta}^{\mathcal{T}}\right)$ and $\mu=\operatorname{cr}\left(E_{\xi}^{\mathcal{T}}\right)$, so $\kappa<\mu$. Since we have translatability at $\delta$, it suffices to see that for each $(\varphi, q)$ there is $\left(\varphi^{\prime}, q^{\prime}\right)$ such that for all $\alpha<\kappa$,

$$
M_{\xi+1}^{\mathcal{T}} \models \varphi(q, \alpha) \Longleftrightarrow M_{\delta}^{\mathcal{T}} \models \varphi^{\prime}\left(q^{\prime}, \alpha\right),
$$

and all $\alpha<\operatorname{cr}\left(E_{\delta_{\zeta}}^{\mathcal{X}}\right)=\omega_{\zeta}(\kappa)$,

$$
M_{\gamma_{\xi+1}}^{\mathcal{X}} \models \varphi\left(\pi_{\xi+1}(q), \alpha\right) \Longleftrightarrow M_{\gamma_{\delta}}^{\mathcal{X}} \models \varphi^{\prime}\left(\pi_{\delta}\left(q^{\prime}\right), \alpha\right) .
$$

Now $\gamma_{\delta} \leq^{\mathcal{X}} \gamma_{\delta \mu}=\operatorname{pred}^{\mathcal{X}}\left(\gamma_{\xi+1}\right) \in I_{\delta}$ and $\left(\gamma_{\delta}, \gamma_{\xi+1}\right]_{\mathcal{X}}$ does not drop in model or degree as $(\delta, \xi+1]_{\mathcal{T}}$ does not. Fix $(\varphi, q)$. As usual, using strong closeness at $\xi$, we can choose $\left(\varphi^{\prime}, q^{\prime}\right)$ such that for all $\alpha<\mu$,

$$
M_{\xi+1}^{\mathcal{T}} \models \varphi(q, \alpha) \Longleftrightarrow M_{\delta}^{\mathcal{T}} \models \varphi^{\prime}\left(q^{\prime}, \alpha\right),
$$

and all $\alpha<\operatorname{cr}\left(E_{\delta_{\xi}}^{\mathcal{X}}\right)=\omega_{\xi}(\kappa)$,

$$
M_{\gamma_{\xi+1}}^{\mathcal{X}} \models \varphi\left(\pi_{\xi+1}(q), \alpha\right) \Longleftrightarrow M_{\gamma_{\xi+1}}^{* \mathcal{X}}=M_{\gamma_{\delta \mu}}^{\mathcal{X}} \models \varphi^{\prime}\left(\pi_{\delta \mu}\left(q^{\prime}\right), \alpha\right) .
$$

But $\pi_{\delta \mu}=i_{\gamma_{\delta} \gamma_{\delta \mu}}^{\mathcal{X}} \circ \pi_{\delta}$ and $\operatorname{cr}\left(E_{\delta_{\zeta}}^{\mathcal{X}}\right)<\operatorname{cr}\left(E_{\delta_{\xi}}^{\mathcal{X}}\right)$, so for all $\alpha<\operatorname{cr}\left(E_{\delta_{\zeta}}^{\mathcal{X}}\right)$, we have

$$
M_{\gamma_{\delta \mu}}^{\mathcal{X}} \models \varphi^{\prime}\left(\pi_{\delta \mu}\left(q^{\prime}\right), \alpha\right) \Longleftrightarrow M_{\gamma_{\delta}}^{\mathcal{X}} \models \varphi^{\prime}\left(\pi_{\delta}\left(q^{\prime}\right), \alpha\right),
$$

so $\left(\varphi^{\prime}, q^{\prime}\right)$ is as desired.

We leave the remaining details to the reader.

4.23 Definition. Let $\Pi^{\prime}:(\mathcal{T}, \theta) \hookrightarrow \mathcal{X}^{\prime}$ and $(\mathcal{X}, \Pi)$ be as in $4.20($ so $\theta<\operatorname{lh}(\mathcal{T})$ ). Then we say that $(\mathcal{X}, \Pi)$ is the one-step copy extension of $\left(\mathcal{X}^{\prime}, \Pi^{\prime}\right)$. 
The second manner of propagating tree embeddings involves the use of an extender in the upper tree $\mathcal{X}^{\prime}$ which is not (considered as) copied from $\mathcal{T}$. We will call such extenders $\mathcal{T}$-inflationary. In this case we can just give the definition directly, as it is clear that it works.

4.24 Definition. Let $\Pi:(\mathcal{T}, \theta) \hookrightarrow \mathcal{X}$ be bounding and $k=\mathrm{u}-\operatorname{deg}(\Pi)$. Let $\gamma_{\alpha}=\gamma_{\Pi \alpha}$, etc. Suppose $\operatorname{lh}(\mathcal{X})=\xi+1$. Let $E \in \mathbb{E}_{+}\left(M_{\xi}^{\mathcal{X}}\right)$ be $\mathcal{X}$-normal. Suppose that the putative $\mathrm{u}-k$-maximal tree $\mathcal{X}^{\prime}=\mathcal{X}^{\wedge}\langle E\rangle$ has wellfounded last model, and let $\eta=\operatorname{pred}^{\mathcal{X}^{\prime}}(\xi+1)$. Suppose that $\eta \in I_{\beta}$ and if $\beta \in \operatorname{lh}(\mathcal{T})^{-}$then $E$ is total over $M_{\eta}^{\mathcal{X}} \mid \operatorname{ind}\left(E_{\eta}^{\Pi}\right)$, and otherwise $E$ is total over $M_{\eta}^{\mathcal{X}}$.

The $E$-inflation of $(\mathcal{X}, \Pi)$ is $\left(\mathcal{X}^{\prime}, \Pi^{\prime}\right)$, where $\Pi^{\prime}:(\mathcal{T}, \beta+1) \hookrightarrow \mathcal{X}^{\prime}$ is the unique tree embedding such that $I_{\Pi^{\prime} \beta}=\left(I_{\beta} \cap \eta+1\right) \cup\{\xi+1\}$ and $I_{\Pi^{\prime} \alpha}=I_{\alpha}$ for every $\alpha<\beta$.

4.25 Remark. The uniqueness of the $E$-inflation is by 4.22 , and existence is easy. We have $P_{\alpha i}^{\prime}=P_{\alpha i}$ and $\pi_{\alpha i}^{\prime}=\pi_{\alpha i}$ for $(\alpha, i) \leq_{\operatorname{lex}}(\beta, 0)$. Because $\Pi$ is bounding, $E_{\xi+1}^{\Pi^{\prime}}$ is $\mathcal{X}^{\prime}$-normal, and if $\operatorname{ind}(E) \leq \operatorname{ind}\left(E_{\xi}^{\Pi}\right)$ or $\eta<\xi$ then $\Pi^{\prime}$ is also bounding.

4.26 Definition. Let $M, \mathcal{T}, \mathcal{X}, k$ be as in 4.7. An almost tree embedding $\Pi$ from $\mathcal{T}$ to $\mathcal{X}$, denoted $\Pi: \mathcal{T} \hookrightarrow_{\text {alm }} \mathcal{X}$, is a system $\Pi$ satisfying the requirements of a tree embedding, except that (letting $\Gamma$ be as in 4.7) we drop the requirement that $\Gamma$ be continuous at limits (but $\Gamma$ must still send limits to limits etc). $\quad-$

4.27 Remark. Note here that if $\operatorname{pred}^{\mathcal{T}}(\beta+1)=\alpha$ and $\alpha$ is a limit, then $\operatorname{pred}^{\mathcal{X}}\left(\gamma_{\beta+1}\right) \in I_{\Pi \alpha}$, and in particular, pred ${ }^{\mathcal{X}}\left(\gamma_{\beta+1}\right) \geq \gamma_{\alpha}$, by the requirements of tree embeddings; this remains a requirement of almost tree embeddings, even when $\Gamma$ is discontinuous at $\alpha$.

Note that given a tree $\mathcal{X}$, the requirements of almost tree embeddings from countable $\mathcal{T} \hookrightarrow$ alm $\mathcal{X}$ are closed in the natural topology, so we can form a tree (in the descriptive set theoretic sense) which searches for a countable $\mathcal{T}$ and almost tree embedding $\Pi: \mathcal{T} \hookrightarrow_{\text {alm }} \mathcal{X}$.

4.28 Lemma. Let $\Pi: \mathcal{T} \hookrightarrow_{\text {alm }} \mathcal{X}$ be an almost tree embedding. Write $\gamma_{\alpha}=$ $\gamma_{\Pi \alpha}$ etc. Then there is a unique tree embedding $\Pi^{\prime}: \mathcal{T} \hookrightarrow \mathcal{X}$ such that $\delta_{\alpha}^{\prime}=\delta_{\alpha}$ for all $\alpha$, where $\delta_{\alpha}^{\prime}=\delta_{\Pi^{\prime} \alpha}$, etc. Hence, for limit $\alpha$,

$$
\gamma_{\alpha}^{\prime}=\sup _{\beta<\alpha} \gamma_{\beta}=\sup _{\beta<\alpha} \delta_{\beta}^{\prime},
$$

whereas for successor $\alpha, \gamma_{\alpha}^{\prime}=\gamma_{\alpha}$ (and $\gamma_{0}^{\prime}=0=\gamma_{0}$ ). Moreover, for each $\alpha$, we have $\omega_{\alpha}=\omega_{\alpha}^{\prime}$ and $\pi_{\alpha}=i_{\gamma_{\alpha}^{\prime} \gamma_{\alpha}}^{\mathcal{X}} \circ \pi_{\alpha}^{\prime}$.

Proof Sketch. This is straightforward; we just mention the key facts. Uniqueness is by 4.22. Fix a limit $\alpha<\operatorname{lh}(\mathcal{T})$. The main point is that

$$
B_{\alpha}={ }_{\operatorname{def}}\left\{\gamma_{\beta} \mid \beta<^{\mathcal{T}} \alpha\right\} \subseteq\left[0, \gamma_{\alpha}\right)_{\mathcal{X}},
$$

so $\gamma_{\alpha}^{\prime}=\sup \left(B_{\alpha}\right) \leq^{\mathcal{X}} \gamma_{\alpha}$. Moreover, for each $\beta<<^{\mathcal{T}} \alpha$,

$$
(\beta, \alpha]_{\mathcal{T}} \cap \mathscr{D}^{\mathcal{T}}=\emptyset \Longleftrightarrow\left(\gamma_{\beta}, \gamma_{\alpha}\right]_{\mathcal{X}} \cap \mathscr{D}^{\mathcal{X}}=\emptyset ;
$$


therefore, $\left(\gamma_{\alpha}^{\prime}, \gamma_{\alpha}\right]_{\mathcal{X}} \cap \mathscr{D}^{\mathcal{X}}=\emptyset$. Because of commutativity requirements of (almost) tree embeddings, we have

$$
\pi_{\alpha} \circ i_{\beta \alpha}^{\mathcal{T}}=i_{\gamma_{\beta} \gamma_{\alpha}}^{\mathcal{X}} \circ \pi_{\beta}
$$

for sufficiently large $\beta<^{\mathcal{T}} \alpha$. Likewise with $\pi_{\alpha}^{\prime}, \gamma_{\alpha}^{\prime}$ replacing $\pi_{\alpha}, \gamma_{\alpha}$. It follows that $\pi_{\alpha}=i_{\gamma_{\alpha}^{\prime} \gamma_{\alpha}}^{\mathcal{X}} \circ \pi_{\alpha}^{\prime}$ (and note $\left.\sigma_{\alpha}^{\prime}=\sigma_{\alpha}\right)$. As remarked above, if $\operatorname{pred}^{\mathcal{T}}(\beta+1)=\alpha$ and $\xi=\operatorname{pred}^{\mathcal{X}}\left(\gamma_{\beta+1}\right)$ then $\xi \in I_{\alpha}$, hence, $\gamma_{\alpha} \leq^{\mathcal{X}} \xi$, and $\delta_{\beta}^{\prime}=\delta_{\beta}$, so

$$
\delta\left(\mathcal{T}\left\lceil\gamma_{\alpha}\right) \leq \operatorname{cr}\left(E_{\delta_{\beta}}^{\mathcal{X}}\right)=\sigma_{\beta}\left(\operatorname{cr}\left(E_{\beta}^{\mathcal{T}}\right)\right)=\sigma_{\beta}^{\prime}\left(\operatorname{cr}\left(E_{\beta}^{\mathcal{T}}\right)\right),\right.
$$

so everything agrees appropriately in producing $M_{\beta+1}^{\mathcal{T}}$ and $M_{\gamma_{\beta+1}^{\prime}}^{\mathcal{X}}$, with regard to the tree embedding $\Pi^{\prime}$.

\subsection{Inflation}

We now proceed to the definition of an inflation of a normal iteration tree $\mathcal{T}$. This will be a normal tree $\mathcal{X}$ which can be interpreted as being produced by using extenders which are either (i) copied from $\mathcal{T}$, or (ii) $\mathcal{T}$-inflationary. Certain nodes $\alpha<\operatorname{lh}(\mathcal{X})$ will correspond to nodes $f(\alpha)<\ln (\mathcal{T})$, in that there will be a natural tree embedding

$$
\Pi_{\alpha}:(\mathcal{T}, f(\alpha)+1) \hookrightarrow \mathcal{X} \uparrow(\alpha+1),
$$

with $\delta_{\Pi_{\alpha} f(\alpha)}=\alpha$. The set of all such $\alpha$ will be denoted by $C$. For successor $\alpha \in C, \Pi_{\alpha}$ will be produced through one of the two methods we have just described. We will take natural direct limits at limit ordinals $\alpha$. The set $C^{-}$ will consist of those $\alpha \in C$ such that $f(\alpha)+1<\operatorname{lh}(\mathcal{T})$, and note that at such $\alpha$, we have $Q_{\Pi_{\alpha} f(\alpha)} \unlhd M_{\alpha}^{\mathcal{X}}$, and the active extender of $Q_{\Pi_{\alpha} f(\alpha)}$ is a copy of $E_{f(\alpha)}^{\mathcal{T}}$.

4.29 Definition (Inflation). Let either (i) $M$ be a u- $k$-sound seg-pm and $\mathcal{T}, \mathcal{X}$ be u- $k$-maximal trees on $M$, or (ii) $M$ be a wcpm and $\mathcal{T}, \mathcal{X}$ be normal trees on $M$. We say that $\mathcal{X}$ is an inflation of $\mathcal{T}$ iff there is a tuple

$$
\left(t, C, C^{-}, f,\left\langle\Pi_{\alpha}\right\rangle_{\alpha \in C}\right)
$$

with the following properties (which will unique the tuple); we will also define further notation:

1. We have $t: \ln (\mathcal{X})^{-} \rightarrow\{0,1\}$. The value of $t(\alpha)$ indicates the type of $E_{\alpha}^{\mathcal{X}}$, either $\mathcal{T}$-copying (if $t(\alpha)=0$ ) or $\mathcal{T}$-inflationary (if $t(\alpha)=1$ ).

2. $C \subseteq \operatorname{lh}(\mathcal{X})^{26}$ and $C \cap[0, \alpha]_{\mathcal{X}}$ is a closed ${ }^{27}$ initial segment of $[0, \alpha]_{\mathcal{X}}$.

\footnotetext{
${ }^{26}$ If $M$ is a wcpm, it will follow from the overall definition that $C=\operatorname{lh}(\mathcal{X})$, and the conditions regarding $C$ will be trivial (but $C^{-}$is still important).

${ }^{27}$ One could drop the closure requirement here, demanding only that $C \cap[0, \alpha]_{\mathcal{X}}$ is an initial segment of $\mathcal{X}$, and adding to condition 10 the requirement that for limit $\alpha, \alpha \in C$ iff $\left(\sup _{\beta<\mathcal{X}_{\alpha}} f(\beta)\right)<\operatorname{lh}(\mathcal{T})$. Then if a limit $\alpha$ were such that $\alpha \notin C$ but $[0, \alpha)_{\mathcal{X}} \subseteq C$, then $[0, \alpha)_{\mathcal{X}}$ would determine a $\mathcal{T}$-cofinal branch $b$. By demanding that $C \cap[0, \alpha]_{\mathcal{X}}$ be closed, we are demanding that such branches $b$ are already incorporated into $\mathcal{T}$.
} 
3. We have $f: C \rightarrow \operatorname{lh}(\mathcal{T})$ and $C^{-}=\{\alpha \in C \mid f(\alpha)+1<\operatorname{lh}(\mathcal{T})\}$.

4. For $\alpha \in C$ we have $\Pi_{\alpha}:(\mathcal{T}, f(\alpha)+1) \hookrightarrow \mathcal{X} \uparrow(\alpha+1)$, with $\delta_{\alpha ; f(\alpha)}=\alpha$, where we write $\delta_{\alpha ; \beta}=\delta_{\Pi_{\alpha} \beta}$, etc.

5. $0 \in C$ and $f(0)=0$ and $\Pi_{0}:(\mathcal{T}, 1) \hookrightarrow \mathcal{X}\lceil 1$ is trivial (see 4.18).

6. Let $\alpha+1<\operatorname{lh}(\mathcal{X})$. Then:

- If $\alpha \in C^{-}$then $\operatorname{ind}\left(E_{\alpha}^{\mathcal{X}}\right) \leq \operatorname{ind}\left(E_{\alpha}^{\Pi_{\alpha}}\right) .{ }^{28}$

$-t(\alpha)=0$ iff $\left[\alpha \in C^{-}\right.$and $\left.E_{\alpha}^{\mathcal{X}}=E_{\alpha}^{\Pi_{\alpha}}\right] \cdot{ }^{29}$

7. Let $\alpha+1<\operatorname{lh}(\mathcal{X})$ be such that $t(\alpha)=0$. Then we interpret $E_{\alpha}^{\mathcal{X}}=E_{\alpha}^{\Pi_{\alpha}}$ as a copy from $\mathcal{T}$, as follows:

$-\alpha+1 \in C$ and $f(\alpha+1)=f(\alpha)+1$.

$-\left(\mathcal{X}\left\lceil\alpha+2, \Pi_{\alpha+1}\right)\right.$ is the one-step copy extension of $\left(\mathcal{X}\left\lceil\alpha+1, \Pi_{\alpha}\right)\right.$.

8. Let $\alpha+1<\operatorname{lh}(\mathcal{X})$ be such that $t(\alpha)=1$. We interpret $E_{\alpha}^{\mathcal{X}}$ as $\mathcal{T}$ inflationary, as follows. Let $\eta=\operatorname{pred}^{\mathcal{X}}(\alpha+1)$. Then:

$-\alpha+1 \in C$ iff $\left[\eta \in C\right.$ and if $M$ is a seg-pm then $\left.Q_{\eta ; \eta^{\prime}} \unlhd M_{\alpha+1}^{* \mathcal{X}}\right]$.

- If $\alpha+1 \in C$ then:

$-f(\alpha+1)=f(\eta)$.

- $\left(\mathcal{X}\left\lceil\alpha+2, \Pi_{\alpha+1}\right)\right.$ is the $E_{\alpha}^{\mathcal{X}}$-inflation of $\left(\mathcal{X}\left\lceil\alpha+1, \Pi_{\eta}\right)\right.$.

9. Let $\alpha \in C$ and $\beta \in I_{\alpha ; \gamma}$ for some $\gamma \leq f(\alpha)$. Then:

$-\beta \in C$ and $f(\beta)=\gamma$.

- $I_{\alpha ; \varepsilon}=I_{\beta ; \varepsilon}$ for all $\varepsilon<f(\beta)=\gamma$,

- $I_{\beta ; f(\beta)}=I_{\alpha ; f(\beta)} \cap(\beta+1)$.

10. If $\alpha \in C$ is a limit ${ }^{30}$ then $f(\alpha)=\sup _{\beta<\mathcal{X}_{\alpha}} f(\beta)$.

4.30 Remark. We make some remarks regarding this definition (literally in the context of seg-pms), continuing with notation as above.

Note first that $\Pi_{\alpha}$ is bounding for each $\alpha \in C$.

Adopt the hypotheses and notation of condition 9 (so $\varepsilon<f(\beta)=\gamma$ ). Note

$$
I_{\alpha ; \varepsilon i}=I_{\beta ; \varepsilon i} \text { and } P_{\alpha ; \varepsilon i}=P_{\beta ; \varepsilon i} \text { and } \pi_{\alpha ; \varepsilon i}=\pi_{\beta ; \varepsilon i} \text { for all } i \text {, }
$$

\footnotetext{
${ }^{28}$ This condition could be dropped, but in our applications it will hold, and it simplifies some things. It ensures that each $\Pi_{\beta}$ is bounding.

${ }^{29}$ If we had required that $t$ be given from the outset (calling the pair $(\mathcal{X}, t)$ an inflation), then this condition could also be weakened to say that if $t(\alpha)=0$ then $\alpha \in C^{-}$and $E_{\alpha}^{\mathcal{X}}=E_{\alpha}^{\Pi_{\alpha}}$. But having the stronger condition also simplifies things and ensures the canonicity of inflations.

${ }^{30}$ Note that by condition 2 , if $\alpha$ is a limit then $\alpha \in C$ iff $[0, \alpha)_{\mathcal{X}} \subseteq C$.
} 
and also $P_{\alpha ; f(\beta) 0}=P_{\beta ; f(\beta) 0}$ and $\pi_{\alpha ; f(\beta)}=\pi_{\beta ; f(\beta)}$. And by 4.14 , if $\widetilde{\beta} \leq^{\mathcal{X}} \alpha$ then $\widetilde{\beta} \in I_{\alpha ; \widetilde{\gamma}}$ for some $\widetilde{\gamma} \leq f(\alpha)$, so condition 9 applies to $\widetilde{\beta}, \widetilde{\gamma}$, and therefore $f(\widetilde{\beta})=\widetilde{\gamma} \leq^{\mathcal{T}} f(\alpha)$

We point out some facts regarding limit stages. Let $\alpha \in C$ be a limit such that $f(\alpha)>f(\beta)$ for all $\beta<{ }^{\mathcal{X}} \alpha$. Note that by condition 9 and the remarks above, for $\xi<f(\alpha)$, we have $I_{\alpha ; \xi}=\lim _{\beta<\mathcal{X}_{\alpha}} I_{\beta ; \xi}$ (where this limit exists in the eventually constant sense) and so likewise for $I_{\alpha ; \xi i}, P_{\alpha ; \xi i}$ and $\pi_{\alpha ; \xi i}$. So

$$
\alpha=\left(\lim _{\xi<f(\alpha)} \gamma_{\alpha ; \xi}\right)=\gamma_{\alpha ; f(\alpha)}=\delta_{\alpha ; f(\alpha)},
$$

so $I_{\alpha ; f(\alpha)}=[\alpha, \alpha]$, determining $\pi_{\alpha ; f(\alpha)}$, etc.

Now let $\alpha \in C$ be a limit such that $f(\alpha)=f(\beta)$ for some $\beta<{ }^{\mathcal{X}} \alpha$. For such $\beta$ we have $\gamma_{\alpha ; f(\alpha)}=\gamma_{\beta ; f(\alpha)}$. We also have $\delta_{\alpha ; f(\alpha)}=\alpha$. This determines the remaining objects $\left(I_{\alpha ; f(\alpha) i}\right.$, etc); they are just the natural direct limits.

Using 4.22, it is easily verified that $\mathcal{T}, \mathcal{X}$ determines $\left(t, C, C^{-}, f, \vec{\Pi}\right)$ :

4.31 Lemma. Let $\mathcal{X}$ be an inflation of $\mathcal{T}$, witnessed by $w=\left(t, C, C^{-}, f, \vec{\Pi}\right)$, and also by $w^{\prime}=\left(t^{\prime}, C^{\prime},\left(C^{-}\right)^{\prime}, f^{\prime}, \vec{\Pi}^{\prime}\right)$. Then $w=w^{\prime}$.

4.32 Definition. Let $\mathcal{X}$ be an inflation of $\mathcal{T}$ as witnessed by $\left(t, C, C^{-}, f, \vec{\Pi}\right)$. Then we write $\left(t, C, C^{-}, f, \vec{\Pi}\right)^{\mathcal{T} \rightsquigarrow \mathcal{X}}=\left(t, C, C^{-}, f, \vec{\Pi}\right)$. For $\alpha \in C^{-}$we write $E_{\alpha}^{\mathcal{T} \rightsquigarrow \mathcal{X}}={ }_{\text {def }} E_{\alpha}^{\Pi_{\alpha}}$.

We may freely extend inflations at successor stages, given wellfoundedness:

4.33 Lemma. Let $\mathcal{X}$ be an inflation of $\mathcal{T}$, with $\operatorname{lh}(\mathcal{X})=\beta+1$. Let $C^{-}=$ $\left(C^{-}\right)^{\mathcal{T} \rightsquigarrow \mathcal{X}}$. Then:

1. If $\beta \in C^{-}$then $E_{\beta}^{\mathcal{T} \rightsquigarrow \mathcal{X}}$ is $\mathcal{X}$-normal.

2. Let $E \in \mathbb{E}_{+}\left(M_{\beta}^{\mathcal{X}}\right)$ be $\mathcal{X}$-normal, with $\operatorname{ind}(E) \leq \operatorname{ind}\left(E_{\beta}^{\mathcal{T} \rightsquigarrow \mathcal{X}}\right)$ if $\beta \in C^{-}$.

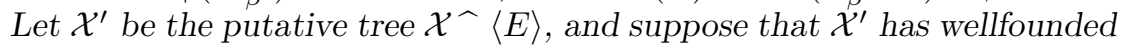
last model. Then $\mathcal{X}^{\prime}$ is an inflation of $\mathcal{T}$.

Proof. Part 1 follows from 4.17, and part 2 from 4.20 (see 4.23 and 4.24).

However, at limit stages, we need to assume some condensation holds of $\Sigma$, in order to extend. This is critical to our purposes, and we consider it next.

\subsection{Inflation condensation and strong hull condensation}

4.34 Remark. Suppose that $\mathcal{X}$, of limit length $\alpha$, is an inflation of $\mathcal{T}$, as witnessed by $(C, f, \ldots)$. Let $b$ be a wellfounded $\mathcal{X}$-cofinal branch, and $\mathcal{X}^{\prime}=$ $\mathcal{X}^{\wedge} b$. We want to see whether $\mathcal{X}^{\prime}$ is an inflation of $\mathcal{T}$. Let $\left(C^{\prime}, f^{\prime}\right)$ be the unique candidate for $(C, f)^{\mathcal{T} \rightsquigarrow \mathcal{X}^{\prime}}$ determined by 4.29. Suppose that $\alpha \in C^{\prime}$ and $f^{\prime}(\beta)<f^{\prime}(\alpha)$ for all $\beta<\mathcal{X}^{\prime} \alpha$ (this is the important case); in particular, $f^{\prime}(\alpha)$ is a limit. Note that $b$ determines a $\mathcal{T} \uparrow f^{\prime}(\alpha)$-cofinal branch $c=f^{\prime \prime} b$, and $\mathcal{X}^{\prime}$ is an inflation of $\mathcal{T}$ iff $c=\left[0, f^{\prime}(\alpha)\right)_{\mathcal{T}}$. 
We first give the definition of inflation condensation for the case that $\Sigma$ is an $(m, \Omega+1)$-strategy for an $m$-sound $\lambda$-indexed premouse $M$, where $\Omega$ is an uncountable regular cardinal. After that, we define in 4.36 some general kinds of (partial) iteration strategies $\Sigma$ we wish to consider, and then give the general definition of inflation condensation for such strategies.

4.35 Definition. Let $\Omega>\omega$ be regular. Let $\Sigma$ be an $(m, \Omega+1)$-strategy for an $m$-sound $\lambda$-indexed $\mathrm{pm} M$. Then $\Sigma$ has inflation condensation or is inflationary iff for all trees $\mathcal{T}, \mathcal{X}$, if

$-\mathcal{T}, \mathcal{X}$ are via $\Sigma$,

$-\mathcal{X}$ is an inflation of $\mathcal{T}$, as witnessed by $(f, C, \ldots)$,

$-\mathcal{X}$ has limit length and $\operatorname{lh}(\mathcal{X}) \leq \Omega$,

$-b=_{\operatorname{def}} \Sigma(\mathcal{X}) \subseteq C$ and $f$ " $b$ has limit ordertype,

then letting $\eta=\sup f$ " $b$, we have $f " b=\Sigma(\mathcal{T}\lceil\eta)$.

For strategies for wcpms, inflation condensation is totally analogous. For strategies $\Lambda$ for MS-indexed mice, we must instead translate $\Lambda$ to the corresponding u-strategy $\Sigma$. We would also like to consider partial strategies (such as a short tree strategy). So we next give an abstract definition of the the kinds of (partial) strategies we wish to consider for inflation condensation in general.

4.36 Definition. Let $M$ be a premouse or wcpm. An iteration class (for $M)$ is a class $\mathscr{T}$ of putative trees on $M$, which is closed under initial segment. Let $\mathscr{T}$ be an iteration class for $M$. A putative partial $\mathscr{T}$-strategy (for $M)$ is a class function $\Sigma$ with $D={ }_{\text {def }} \operatorname{dom}(\Sigma)$ such that $D \subseteq \mathscr{T}$, and for each $\mathcal{T} \in D, \mathcal{T}$ has limit length, $\Sigma(\mathcal{T})$ is a $\mathcal{T}$-cofinal branch and $\mathcal{T}^{\wedge} \Sigma(\mathcal{T}) \in \mathscr{T}$. Let $\Sigma$ be a putative partial $\mathscr{T}$-strategy. For $\mathcal{T} \in \mathscr{T}$, we say that $\mathcal{T}$ is via $\Sigma$ iff $\mathcal{T}\left\lceil\eta \in \operatorname{dom}(\Sigma)\right.$ and $[0, \eta)_{\mathcal{T}}=\Sigma(\mathcal{T}\lceil\eta)$ for every limit $\eta<\operatorname{lh}(\mathcal{T})$. We say that $\Sigma$ is a partial $\mathscr{T}$-strategy iff every $\mathcal{T} \in \mathscr{T}$ via $\Sigma$ has wellfounded well-defined models.

Given $M, \Sigma$, we say that $\Sigma$ is a (putative) partial pre-inflationary strategy (for $M$ ) iff $\Sigma$ is a (putative) partial $\mathscr{T}$-strategy (for $M$ ), where for some $m \leq \omega$, either

(i) $M$ is a wcpm, $m=0$ and $\mathscr{T}$ is the class of putative normal trees on $M$, or

(ii) $M$ is a $\mathrm{u}-m$-sound seg-pm and $\mathscr{T}$ is the class of putative $\mathrm{u}-m$-maximal trees on $M$, or

(iii) $M$ is an $m$-sound MS-indexed pm and $\mathscr{T}$ is the class of putative $m$ maximal trees on $M$. 
We say that $\Sigma$ is conveniently pre-inflationary iff either (i) or (ii) above hold, and inconveniently pre-inflationary iff (iii) holds. ${ }^{31}$

Let $\Sigma$ be pre-inflationary and $\mathscr{T}, M, m$ be as in the preceding paragraph. We say that $\Sigma$ is regularly $\Xi$-total iff there is an regular uncountable $\Omega$ such that $\Omega \leq \Xi \leq \Omega+1$ and $\Sigma$ is either a normal $\Xi$-strategy for a wcpm (and $m=0)$, a $(\mathrm{u}-m, \Xi)$-strategy, or an $(m, \Xi)$-strategy for an MS-indexed $M$. In this case we write $m^{\Sigma}=m$.

We can now give the general definition of inflation condensation.

4.37 Definition (Inflation condensation). Let $\Sigma$ be a conveniently pre-inflationary partial strategy. Then $\Sigma$ has convenient inflation condensation or is conveniently inflationary iff for all trees $\mathcal{T}, \mathcal{X}$, if

$-\mathcal{T}, \mathcal{X}$ are via $\Sigma$

$-\mathcal{X}$ is an inflation of $\mathcal{T}$, as witnessed by $(f, C, \ldots)$,

- $\mathcal{X}$ has limit length and $\mathcal{X} \in \operatorname{dom}(\Sigma)$,

$-b=_{\operatorname{def}} \Sigma(\mathcal{X}) \subseteq C$ and $f$ " $b$ has limit ordertype,

then letting $\eta=\sup f$ " $b$, we have $\mathcal{T}\left\lceil\eta \in \operatorname{dom}(\Sigma)\right.$ and $f^{\prime \prime} b=\Sigma(\mathcal{T}\lceil\eta)$.

Let $\Lambda$ be an inconveniently pre-inflationary partial strategy. Let $\Sigma$ be the partial u-strategy corresponding to $\Lambda$ (as in Remark 2.16, which applies by Footnote 31). Then $\Lambda$ has inconvenient inflation condensation or is inconveniently inflationary iff $\Sigma$ is conveniently inflationary.

In general, we say that $\Sigma$ (or $\Lambda$ ) has inflation condensation or is inflationary iff $\Sigma$ (or $\Lambda$ ) has convenient or inconvenient inflation condensation. $\dashv$

Immediately from the definition, inflations via inflationary $\Sigma$ can be continued at limit stages:

4.38 Lemma. Let $\Sigma$ be a conveniently inflationary partial strategy. Let $\mathcal{T}, \mathcal{X}$ be such that $\mathcal{X}$ is via $\Sigma, \mathcal{X}$ is an inflation of $\mathcal{T}$, as witnessed by $(f, C, \ldots)$, and

$$
\operatorname{lh}(\mathcal{T})=\sup _{\alpha \in C}(f(\alpha)+1)
$$

Then $\mathcal{T}$ is via $\Sigma$.

Suppose also that $\mathcal{X}$ has limit length $\lambda$ and $\mathcal{X} \in \operatorname{dom}(\Sigma)$, and let $\mathcal{X}^{\prime}=$ $(\mathcal{X}, \Sigma(\mathcal{X}))$. Then there is $\mathcal{T}^{\prime}$ via $\Sigma$ such that $\mathcal{T} \unlhd \mathcal{T}^{\prime}$ and $\mathcal{X}^{\prime}$ is an inflation of $\mathcal{T}^{\prime}$, as witnessed by $\left(C^{\prime}, f^{\prime}, \ldots\right)$. Moreover, we may take $\mathcal{T}^{\prime}$ such that either:

$$
-\mathcal{T}^{\prime}=\mathcal{T} \text { and if } \lambda \in C^{\prime} \text { then } f^{\prime}(\lambda)<\ln (\mathcal{T}) \text {, or }
$$

\footnotetext{
${ }^{31}$ Let $\Sigma$ be partial pre-inflationary. Note that there can be limit length trees $\mathcal{T} \in \mathscr{T}$ which are via $\Sigma$, but with $\mathcal{T} \notin D$. And if (ii) or (iii) holds and $\mathcal{T} \in \mathscr{T}$ is via $\Sigma$ of successor length, then $M_{\infty}^{\mathcal{T}}$ is well-defined and wellfounded, and all $\mathrm{u}-m$-maximal $/ m$-maximal putative trees $\mathcal{T}^{\prime}$ such that $\mathcal{T} \unlhd \mathcal{T}^{\prime}$ and $\operatorname{lh}\left(\mathcal{T}^{\prime}\right)<\operatorname{lh}(\mathcal{T})+\omega$, are also in $\mathscr{T}$ and via $\Sigma$, and hence have wellfounded models. Therefore if (ii) holds where $M$ is MS-indexed then $\mathcal{T}$ is everywhere unravelable, and if (iii) holds then $\mathcal{T}$ is $M$-u-wellfounded.
} 
- $\mathcal{T}$ has limit length $\bar{\lambda}, \mathcal{T}^{\prime}=(\mathcal{T}, \Sigma(\mathcal{T})), \lambda \in C^{\prime}, f^{\prime}(\lambda)=\bar{\lambda}$ and $\gamma_{\lambda ; \bar{\lambda}}^{\prime}=\lambda$.

Further, the choice of $\mathcal{T}^{\prime}$ is uniqued by adding these requirements.

We also immediately have:

4.39 Lemma. Let $\Sigma$ be a conveniently inflationary partial strategy and $\mathcal{T}, \mathcal{X}$ be via $\Sigma$. Then $\mathcal{X}$ is an inflation of $\mathcal{T}$ iff:

- $\mathcal{X}$ satisfies the bounding requirements on extender indices imposed by $\mathcal{T}$; that is, for each $\alpha+1<\ln (\mathcal{X})$, if $\mathcal{X} \uparrow(\alpha+1)$ is an inflation of $\mathcal{T}$ and $\alpha \in\left(C^{-}\right)^{\mathcal{T} \rightsquigarrow \mathcal{X} \mid(\alpha+1)}$ then $\operatorname{ind}\left(E_{\alpha}^{\mathcal{X}}\right) \leq \operatorname{ind}\left(E_{\alpha}^{\mathcal{T} \rightsquigarrow(\mathcal{X} \mid \alpha+1)}\right)$, and

- if $\mathcal{T}$ has limit length then $\mathcal{X}$ does not determine a $\mathcal{T}$-cofinal branch; that is, if $\eta<\operatorname{lh}(\mathcal{X})$ is a limit and $\mathcal{X}\lceil\eta$ is an inflation of $\mathcal{T}$ and $(f, C)=$ $(f, C)^{\mathcal{T} \rightsquigarrow \mathcal{X} \mid \eta}$ and $[0, \eta)_{\mathcal{X}} \subseteq C$ then $\ln (\mathcal{T})>\sup _{\alpha<{ }^{\mathcal{X}} \eta} f(\alpha)$.

4.40 Definition. Let $\Sigma$ be a partial iteration strategy and $\mathcal{T}$ be via $\Sigma$, with $\mathcal{T}$ either of successor length or $\mathcal{T} \in \operatorname{dom}(\Sigma)$. Then $\operatorname{complete}^{\Sigma}(\mathcal{T})$ denotes $\mathcal{T}$ if $\ln (\mathcal{T})$ is a successor, and denotes $\mathcal{T}^{\wedge} \Sigma(\mathcal{T})$ otherwise.

We easily have:

4.41 Lemma. Let $\Sigma$ be a conveniently inflationary partial strategy. Let $\mathcal{T}, \mathcal{X}$ be via $\Sigma$, with $\mathcal{X}$ an inflation of $\mathcal{T}, \mathcal{X}$ of limit length, $\mathcal{X} \in \operatorname{dom}(\Sigma)$. Then either complete ${ }^{\Sigma}(\mathcal{X})$ is an inflation of $\mathcal{T}$, or $\mathcal{T}$ has limit length, $\mathcal{T} \in \operatorname{dom}(\Sigma)$ and $\operatorname{complete}^{\Sigma}(\mathcal{X})$ is an inflation of $\operatorname{complete}^{\Sigma}(\mathcal{T})$.

Steel uses the following notion of strategy condensation in [21] (however, note we also allow partial strategies). It easily implies inflation condensation; we do not know whether the converse holds.

4.42 Definition. Let $\Sigma$ be a conveniently pre-inflationary partial strategy. We say that $\Sigma$ has convenient strong hull condensation iff whenever $\mathcal{X}$ is via $\Sigma$ and $\Pi: \mathcal{T} \hookrightarrow \mathcal{X}$ is a tree embedding, then $\mathcal{T}$ is also via $\Sigma$.

Let $\Lambda$ be an inconveniently pre-inflationary partial strategy. We say that $\Lambda$ has inconvenient strong hull condensation iff whenever $\Sigma$ has convenient strong hull condensation, where $\Sigma$ is the partial u-strategy corresponding to $\Lambda$.

We say that a pre-inflationary partial strategy has strong hull condensation iff it has either convenient or inconvenient strong hull condensation.

A third condensation notion, also a consequence of strong hull condensation, we will make use of in $\S 7$ in our generic absoluteness argument. For our normal realization results we only require inflation condensation.

4.43 Definition. Let $\Sigma$ be a conveniently pre-inflationary partial strategy. We say that $\Sigma$ is conveniently extra inflationary iff $\Sigma$ is conveniently inflationary and for all sufficiently large $\theta \in \mathrm{OR}$, for all countable transitive $X$ and elementary

$$
\pi: X \rightarrow \mathcal{H}_{\theta}
$$

and $\overline{\mathcal{T}} \in X$ such that $\pi(\overline{\mathcal{T}})$ is via $\Sigma$, (so $\overline{\mathcal{T}}$ is on $\bar{M}$ where $\pi(\bar{M})=M$ ), then $\pi \overline{\mathcal{T}}$ (the copy of $\overline{\mathcal{T}}$ to $M$ via $\pi$ ) is via $\Sigma$. 
We then define inconveniently extra inflationary, and extra inflationary, as before.

4.44 Lemma. If $\Sigma$ has strong hull condensation then $\Sigma$ is extra inflationary.

Proof. The fact that $\Sigma$ has inflation condensation is immediate (inflation condensation just requires the $\Sigma$ condenses under the tree embeddings which arise from inflation).

So let $\pi: X \rightarrow \mathcal{H}_{\theta}$ and $\overline{\mathcal{T}} \in X$ be as in 4.43 and $\mathcal{T}=\pi(\overline{\mathcal{T}})$. We will observe that $\pi \overline{\mathcal{T}}$ is via $\Sigma$. We define an almost tree embedding

$$
\Pi: \pi \overline{\mathcal{T}} \hookrightarrow \text { alm } \mathcal{T}
$$

by setting $I_{\alpha}=\left[\gamma_{\alpha}, \delta_{\alpha}\right]_{\mathcal{T}}=[\pi(\alpha), \pi(\alpha)]_{\mathcal{T}}$. One verifies by a straightforward induction on $\theta \leq \operatorname{lh}(\overline{\mathcal{T}})$ that

$$
\Pi \uparrow(\theta+1):(\pi \overline{\mathcal{T}} \uparrow(\theta+1)) \hookrightarrow_{\text {alm }} \mathcal{T}
$$

is an almost tree embedding, with associated maps $\pi_{\alpha}$ and $\omega_{\alpha}=\pi_{\alpha} \uparrow \operatorname{ex}_{\alpha}^{\mathcal{T}}$, and letting $\varrho_{\alpha}: M_{\alpha}^{\overline{\mathcal{T}}} \rightarrow M_{\alpha}^{\pi \overline{\mathcal{T}}}$ be the copy map induced by $\pi: \bar{M} \rightarrow M$, that $\pi_{\alpha} \circ \varrho_{\alpha}=\pi \uparrow M_{\alpha}^{\overline{\mathcal{T}}}$, and hence,

$$
E_{\delta_{\alpha}}^{\mathcal{\mathcal { T }}}=E_{\pi(\alpha)}^{\mathcal{\mathcal { T }}}=\pi\left(E_{\alpha}^{\overline{\mathcal{T}}}\right)=\pi_{\alpha}\left(\varrho_{\alpha}\left(E_{\alpha}^{\overline{\mathcal{T}}}\right)\right)=\pi_{\alpha}\left(E_{\alpha}^{\pi \overline{\mathcal{T}}}\right)
$$

This is routine and we leave it to the reader.

By 4.28 and strong hull condensation, it follows that $\overline{\mathcal{T}}$ is according to $\Sigma$.

We now give some important examples of strategies with strong hull condensation.

4.45 Lemma. Let $\Sigma$ be a regularly $\Xi$-total pre-inflationary strategy for $M$, and suppose that $\Sigma$ is the unique such strategy for $M$. Then $\Sigma$ has strong hull condensation.

Proof. We leave the wcpm case to the reader. Consider the fine case. It suffices then to consider the case that $\Sigma$ is convenient, by the 1-1 correspondence between u-strategies and standard strategies for MS-indexed premice (see 2.13).

Let $\Pi:(\mathcal{T}, c) \hookrightarrow(\mathcal{X}, d)$ be a tree embedding, with $(\mathcal{X}, d)$ via $\Sigma, \mathcal{T}, \mathcal{X}$ of limit length, $c$ is $\mathcal{T}$-cofinal. We may assume that $\mathcal{T}$ is via $\Sigma$ and $\Pi$ is cofinal in $\operatorname{lh}(\mathcal{X})$. We must show that $c=\Sigma(\mathcal{T})$. Let $\eta=\operatorname{lh}(\mathcal{T})$.

If $\eta=\Omega={ }_{\text {def }} \Omega^{\Sigma}$ this holds because $\operatorname{cof}(\Omega)>\omega$. So suppose $\eta<\Omega$. Then $\operatorname{lh}(\mathcal{X})<\Omega$ because $\Pi$ is cofinal. And $k={ }_{\operatorname{def}} \mathrm{u}-\operatorname{deg}^{\mathcal{X}}(d)=\mathrm{u}-\operatorname{deg}^{\mathcal{T}}(c)$. By the uniqueness of $\Sigma$, it suffices to see that the phalanx $\Phi(\mathcal{T}, c)$ is (u- $k, \Xi)$-iterable. But using the embeddings given by $\Pi$, we can copy u- $k$-maximal trees on $\Phi(\mathcal{T}, c)$ to $\mathrm{u}$ - $k$-maximal trees on $\Phi(\mathcal{X}, d) .{ }^{32}$ Since $(\mathcal{X}, d)$ is via $\Sigma$ and $\operatorname{lh}(\mathcal{X})<\Omega$, this suffices.

\footnotetext{
${ }^{32}$ Use the one-step copy extension at successor stages and form direct limits at limit stages.
} 
4.46 Remark. The previous lemma can be adapted to wcpms in the obvious manner. However, we do not see how to adapt the following theorem to wcpms, because it relies on a comparison argument. Recall from [5] or [24] the weak Dodd-Jensen property for an iteration strategy $\Sigma$ for a countable premouse $M$. John Steel pointed out the following theorem (or something very similar, and in the case that $M$ is $\lambda$-indexed) to the author in 2017. We note that a variant of its proof shows that if $\Omega>\omega$ is regular, and $e$ an enumeration of $M$ in ordertype $\omega$, there is at most one $(m, \Omega+1)$-strategy $\Sigma$ for $M$ with weak Dodd-Jensen with respect to $e$. We often abbreviate Dodd-Jensen with $D J$.

4.47 Theorem. Let $\Omega>\omega$ be regular. Let $M$ be an $m$-sound premouse with $\operatorname{card}(M)<\Omega$. Let $\Sigma$ be an $(m, \Omega+1)$-strategy for $M$ such that either $\Sigma$ has the DJ property, or $M$ is countable and $\Sigma$ has weak DJ. Then $\Sigma$ has strong hull condensation.

Proof. We literally assume that $M$ is countable and $\Sigma$ has weak DJ; otherwise it is almost the same but slightly simpler.

We consider first the case that $M$ is $\lambda$-indexed. Thus, $m$ - and $u-m$ - fine structure are equivalent. Suppose the theorem fails in this case, and let

$$
\Pi:(\mathcal{T}, c) \hookrightarrow(\mathcal{X}, d)
$$

be a tree embedding, with properties as before. Let $b=\Sigma(\mathcal{T})$ and suppose that $b \neq c$. We have $\ln (\mathcal{T}), \operatorname{lh}(\mathcal{X})<\Omega$ as $\Omega>\omega$ is regular.

Let $\Gamma$ be the $(\Omega+1)$-strategy for $\Phi(\mathcal{T}, c)$ induced by lifting to $\Phi(\mathcal{X}, d)$. Let $\Sigma^{\prime}$ be the $(\Omega+1)$-strategy for $\Phi(\mathcal{T}, b)$ induced by $\Sigma$. Because $M$ and $\mathcal{T}$ have cardinality $<\Omega$, we get a successful comparison $(\mathcal{U}, \mathcal{V})$ extending $((\mathcal{T}, b),(\mathcal{T}, c))$, according to $\Sigma, \Gamma$; here $\mathcal{U}, \mathcal{V}$ are $m$-maximal trees on $M$. (Note that ZF suffices here; although the standard proof the comparison terminates involves taking a hull of $V$, we can do this part working inside $L[X]$ where $X \subseteq$ OR codes the comparison.) Let $\mathcal{W}$ be the tree extending $\mathcal{X}$, which is the lift of $\mathcal{V}$. Let $\pi_{\infty}: M_{\infty}^{\mathcal{V}} \rightarrow M_{\infty}^{\mathcal{W}}$ be the final lifting map.

If $M_{\infty}^{\mathcal{V}} \triangleleft M_{\infty}^{\mathcal{U}}$ then $b^{\mathcal{V}}$ does not drop, so $\mathcal{U}$ and $i_{\infty}^{\mathcal{V}}: M \rightarrow M_{\infty}^{\mathcal{V}}$ contradicts weak DJ for $\Sigma$; likewise if $b^{\mathcal{U}}$ drops in model or degree but $b^{\mathcal{V}}$ does not. If $M_{\infty}^{\mathcal{U}} \triangleleft M_{\infty}^{\mathcal{V}}$ then $\pi_{\infty}\left(M_{\infty}^{\mathcal{U}}\right) \triangleleft M_{\infty}^{\mathcal{W}}$, so $\mathcal{W}$ and $\pi_{\infty} \circ i_{\infty}^{\mathcal{U}}$ contradicts weak DJ; likewise if $b^{\mathcal{V}}$ drops in model or degree (and hence $b^{\mathcal{W}}$ drops correspondingly) but $b^{\mathcal{U}}$ does not. So $M_{\infty}^{\mathcal{U}}=M_{\infty}^{\mathcal{V}}$ and neither $b^{\mathcal{U}}$ nor $b^{\mathcal{V}}$ drops in model or degree.

We claim that $i^{\mathcal{U}}=i^{\mathcal{V}}$. For suppose not. Let $\left\langle x_{i}\right\rangle_{i<\omega}$ be our enumeration of $M$ relative to which $\Sigma$ has the weak DJ property. Let $k$ be least such that $i^{\mathcal{U}}\left(x_{k}\right) \neq i^{\mathcal{V}}\left(x_{k}\right)$. Since $i^{\mathcal{V}}$ is a near $n$-embedding, and $\mathcal{U}$ is according to $\Sigma$, weak DJ gives $i^{\mathcal{U}}\left(x_{k}\right)<i^{\mathcal{V}}\left(x_{k}\right)$. But since $b^{\mathcal{V}}$ does not drop, $\pi_{\infty}$ is also a near $n$-embedding, so $\pi_{\infty} \circ i^{\mathcal{U}}$ is likewise, as is $i^{\mathcal{W}}$, and $i^{\mathcal{W}}=\pi_{\infty} \circ i^{\mathcal{V}}$. Therefore $\pi_{\infty}\left(i^{\mathcal{U}}\left(x_{k}\right)\right)<\pi_{\infty}\left(i^{\mathcal{V}}\left(x_{k}\right)\right)=i^{\mathcal{W}}\left(x_{k}\right)$, so we contradict weak DJ with $\mathcal{W}$ (which is according to $\Sigma$ ) and $\pi_{\infty} \circ i^{\mathcal{U}}$.

So $i^{\mathcal{U}}=i^{\mathcal{V}}$. Using this, standard fine structural calculations yield a contradiction. Here is a reminder. One first shows that $b^{\mathcal{U}}$ extends $b$ and $b^{\mathcal{V}}$ extends 
$c$. Then, let $\gamma=\max (b \cap c)$, so $\gamma<\operatorname{lh}(\mathcal{T})$. Let $\nu=\sup _{\alpha<\gamma} \nu\left(E_{\alpha}^{\mathcal{T}}\right)$. Then

$$
M_{\gamma}^{\mathcal{T}}=\operatorname{cHull}_{n+1}^{M_{\infty}^{\mathcal{U}}}\left(\operatorname{rg}\left(i^{\mathcal{U}}\right) \cup \nu\right)
$$

and $i_{\gamma \infty}^{\mathcal{U}}$ is just the uncollapse map. Likewise with $\mathcal{V}$ replacing $\mathcal{U}$. But then $i_{\gamma \infty}^{\mathcal{U}}=i_{\gamma \infty}^{\mathcal{V}}$, which contradicts the fact that $\gamma=\max (b \cap c)$. This completes the proof in this case.

Now suppose instead that $M$ is MS-indexed. Thus, the statement that $\Sigma$ has strong hull condensation literally means that $\Sigma^{\prime}$ has inflation condensation, where $\Sigma^{\prime}$ is the u-strategy corresponding to $\Sigma$. Suppose the theorem fails in this case, and let

$$
\Pi^{\prime}:\left(\mathcal{T}^{\prime}, c^{\prime}\right) \hookrightarrow\left(\mathcal{X}^{\prime}, d^{\prime}\right),
$$

etc, be a counterexample as before, and $b^{\prime}=\Sigma^{\prime}\left(\mathcal{T}^{\prime}\right)$. Again $\operatorname{lh}\left(\mathcal{T}^{\prime}\right), \operatorname{lh}\left(\mathcal{X}^{\prime}\right)<\Omega$.

Let $\Gamma_{c^{\prime}}^{\prime}$ be the $(\Omega+1)$-strategy for $\Phi\left(\mathcal{T}^{\prime}, c^{\prime}\right)$ induced by lifting to $\Phi\left(\mathcal{X}^{\prime}, d^{\prime}\right)$. Let $\Gamma_{b^{\prime}}^{\prime}$ be the $(\Omega+1)$-strategy for $\Phi\left(\mathcal{T}^{\prime}, b^{\prime}\right)$ induced by $\Sigma^{\prime}$.

Now let $\mathcal{T}, \mathcal{X}, c, \Gamma_{c}$, etc, be the canonical conversions of all of these objects to standard MS-premice given by 2.13 and 2.12. (For $c, \Gamma_{c}$, proceed as follows. First define a u-strategy $\widetilde{\Sigma}^{\prime}$ for $M$, by just following $\Sigma^{\prime}$, except that $\widetilde{\Sigma}^{\prime}\left(\mathcal{T}^{\prime}\right)=c^{\prime}$ and $\widetilde{\Sigma}^{\prime}$ proceeds according to $\Gamma_{c^{\prime}}^{\prime}$ for trees extending $\mathcal{T}^{\prime}$. Then let $\widetilde{\Sigma}$ be the $m$-maximal strategy for $M$ corresponding to $\widetilde{\Sigma}^{\prime}$, given by 2.13 . Finally let $c, \Gamma_{c}$ be determined by $\widetilde{\Sigma}$.) Then $\ln (\mathcal{T})=\eta^{\prime}=\operatorname{lh}\left(\mathcal{T}^{\prime}\right)$ (as $\eta^{\prime}$ is a limit), $c \neq b$, and $\Gamma_{c}, \Gamma_{b}$ are $(\Omega+1)$-strategies for $\Phi(\mathcal{T}, c), \Phi(\mathcal{T}, b)$.

We get a successful comparison ${ }^{33}(\mathcal{U}, \mathcal{V})$ extending $((\mathcal{T}, b),(\mathcal{T}, c))$, according to $\Gamma_{b}, \Gamma_{c} ;($ here $\mathcal{U}, \mathcal{V}$ are $m$-maximal trees on $M)$. Let $\mathcal{U}^{\prime}, \mathcal{V}^{\prime}$ be the corresponding u-trees (in the sense of 2.12), so $\left(M_{\infty}^{\mathcal{U}^{\prime}}\right)^{\mathrm{pm}}=M_{\infty}^{\mathcal{U}}$ and $\left(M_{\infty}^{\mathcal{U}}\right)^{\mathrm{pv}} \unlhd\left(M_{\infty}^{\mathcal{U}^{\prime}}\right)^{\mathrm{pv}}$, and if $M_{\infty}^{\mathcal{U}}$ is type 3 and $\operatorname{deg}^{\mathcal{U}}(\infty)<\omega$ then $\mathrm{u}-\operatorname{deg}^{\mathcal{U}^{\prime}}(\infty)=\operatorname{deg}^{\mathcal{U}}(\infty)+1$, and otherwise $M_{\infty}^{\mathcal{U}^{\prime}}=M_{\infty}^{\mathcal{U}}$ and $\mathrm{u}$ - $\operatorname{deg}^{\mathcal{U}^{\prime}}(\infty)=\operatorname{deg}^{\mathcal{U}}(\infty)$. Likewise for $\mathcal{V}, \mathcal{V}^{\prime}$. In particular, $\infty$ is non- $\mathcal{U}^{\prime}$-special and non- $\mathcal{V}^{\prime}$-special.

Let $\mathcal{W}^{\prime}$ be the tree extending $\mathcal{X}^{\prime}$, which is the lift of $\mathcal{V}^{\prime}$; thus, $\mathcal{W}^{\prime}$ is according to $\Sigma^{\prime}$. Then $M_{\infty}^{\mathcal{V}^{\prime}}, M_{\infty}^{\mathcal{\mathcal { W } ^ { \prime }}}$ have the same type and u-deg $\mathcal{V}^{\prime}(\infty)=\mathrm{u}-\operatorname{deg}^{\mathcal{W}^{\prime}}(\infty)$ because cofinally many extenders used in $\mathcal{W}^{\prime}$ are copied from $\mathcal{V}^{\prime}$ (note this includes the case that $\mathcal{V}^{\prime}=\left(\mathcal{T}^{\prime}, b^{\prime}\right)$ ). Thus, $\infty$ is non- $\mathcal{W}^{\prime}$-special. So letting $\mathcal{W}$ be the standard MS-tree corresponding to $\mathcal{W}^{\prime}$, then $\mathcal{W}$ is according to $\Sigma$ and $\left(M_{\infty}^{\mathcal{W}^{\prime}}\right)^{\mathrm{pm}}=M_{\infty}^{\mathcal{W}}$ and $\operatorname{deg}^{\mathcal{W}}(\infty)=\operatorname{deg}^{\mathcal{V}}(\infty)$. Let $\pi_{\infty}^{\prime}: M_{\infty}^{\mathcal{V}^{\prime}} \rightarrow M_{\infty}^{\mathcal{W}^{\prime}}$ be the final copy map. Let $\pi_{\infty}=\pi_{\infty}^{\prime}\left\lceil\left(\left(M_{\infty}^{\mathcal{V}^{\prime}}\right)^{\mathrm{pm}}\right)^{\mathrm{sq}}\right.$. Then $\pi_{\infty}:\left(M_{\infty}^{\mathcal{V}}\right)^{\mathrm{sq}} \rightarrow\left(M_{\infty}^{\mathcal{W}}\right)^{\mathrm{sq}}$ is a near $\operatorname{deg}^{\mathcal{V}}(\infty)$-embedding (as $\pi_{\infty}^{\prime}$ is a near $\mathrm{u}$ - $\operatorname{deg}^{\mathcal{V}^{\prime}}(\infty)$-embedding).

Because we have $\pi_{\infty}$, weak DJ gives that $M_{\infty}^{\mathcal{V}}=M_{\infty}^{\mathcal{W}}$ as usual. We have that $[0, \infty]_{\mathcal{U}}$ drops iff $[0, \infty]_{\mathcal{U}^{\prime}}$ drops (by Lemma 2.12), and if non-dropping, that

\footnotetext{
${ }^{33}$ Here we adjust the algorithm for comparison, for example as in [18], by minimizing on $\nu(E)$ before using an extender $E$. That is, if at stage $\alpha$ of the comparison, the least disagreement consists in two non-empty extenders $E, F$, and $\nu(E) \neq \nu(F)$, then we use $E$ if $\nu(E)<\nu(F)$, padding on the other side, and vice versa if $\nu(F)<\nu(E)$. This avoids the uncomfortable situation of using extender $E$ on side 1 and $F$ on side 2 at stage $\alpha$, where $E$ is superstrong and $F$ type 2 , and then using the same $F$ on side 1 at stage $\alpha+1$.
} 
$i^{\mathcal{U}}=i^{\mathcal{U}^{\prime}}\left\lceil M^{\text {sq }}\right.$; likewise for $\mathcal{V}, \mathcal{V}^{\prime}$ and $\mathcal{W}, \mathcal{W}^{\prime}$. Also, $b^{\mathcal{V}}$ drops iff $b^{\mathcal{W}}$ drops, and if non-dropping, then $\pi_{\infty}^{\prime} \circ i^{\mathcal{V}^{\prime}}=i^{\mathcal{W}^{\prime}}$ and $\pi_{\infty} \circ i^{\mathcal{V}}=i^{\mathcal{W}}$.

With these facts, the usual weak DJ argument leads to contradiction.

\subsection{Further inflation terminology}

4.48 Definition. Let $\mathcal{T}$ be an iteration tree, either $\mathrm{u}-m$-maximal or $m$-maximal, or normal on a wcpm. We say that $\mathcal{T}$ is terminally non-dropping $\operatorname{iff} \ln (\mathcal{T})$ is a successor and if $\mathcal{T}$ is $\mathrm{u}$ - $m$-maximal or $m$-maximal then $b^{\mathcal{T}}$ does not drop in model or degree.

4.49 Definition. Let $\mathcal{X}, \mathcal{T}$ be on $M$, with $\mathcal{X}$ an inflation of $\mathcal{T}$. Let

$$
\left(t, C, C^{-}, f, \vec{\Pi}\right)=\left(t, C, C^{-}, f, \vec{\Pi}\right)^{\mathcal{T} \rightsquigarrow \mathcal{X}}
$$

and let $\gamma_{\alpha ; \beta}$, etc, be as in 4.29. Suppose that $\mathcal{X}$ has successor length $\alpha+1$.

We say that $\mathcal{X}$ is:

- $(\mathcal{T})$-pending iff $\alpha \in C^{-}$.

- non- $(\mathcal{T})$-pending iff $\alpha \notin C^{-}$.

- ( $\mathcal{T})$-terminal iff $\mathcal{T}$ has successor length and $\mathcal{X}$ is non- $\mathcal{T}$-pending.

Suppose that $\mathcal{X}$ is $\mathcal{T}$-terminal. We say that $\mathcal{X}$ is:

- $\mathcal{T}$-terminally-non-model-dropping iff $\alpha \in C$ (hence, $f(\alpha)+1=\operatorname{lh}(\mathcal{T})$ ),

- $\mathcal{T}$-terminally-non-dropping iff $\alpha \in C$ and $\mathrm{u}$ - $\operatorname{deg}^{\mathcal{X}}(\alpha)=\mathrm{u}^{-\operatorname{deg}^{\mathcal{T}}}(f(\alpha))$,

- $\mathcal{T}$-terminally-model-dropping iff $\alpha \notin C$,

- $\mathcal{T}$-terminally-dropping iff $\alpha \notin C$ or u- $\operatorname{deg}^{\mathcal{X}}(\alpha)<\mathrm{u}^{-\operatorname{deg}^{\mathcal{T}}}(f(\alpha))$.

Suppose $\mathcal{X}$ is $\mathcal{T}$-terminally-non-model-dropping and let $\alpha+1=\operatorname{lh}(\mathcal{X})$ and $\beta=f(\alpha)$ and $\gamma=\gamma_{\alpha ; \beta}$. Then we define

$$
\pi_{\infty}^{\mathcal{T} \rightsquigarrow \mathcal{X}}: M_{\beta}^{\mathcal{T}} \rightarrow M_{\alpha}^{\mathcal{X}}
$$

by $\pi_{\infty}^{\mathcal{T} \rightsquigarrow \mathcal{X}}=i_{\gamma \alpha}^{\mathcal{X}} \circ \pi_{\alpha ; \beta}$.

4.50 Remark. Suppose $\mathcal{X}$ is $\mathcal{T}$-terminally-non-model-dropping and $\mathcal{T}, \mathcal{X}$ are $\mathrm{u}$ - $m$-maximal. Note that $\pi_{\infty}=\pi_{\infty}^{\mathcal{T} \rightsquigarrow \mathcal{X}}$ is a near $\mathrm{u}$ - $n$-embedding, where $n=$ $\mathrm{u}$ - $\operatorname{deg}^{\mathcal{X}}(\infty)$. If $\mathcal{X}$ is $\mathcal{T}$-terminally-non-dropping and $\mathcal{T}$ is terminally non-dropping, then note that $\mathcal{X}$ is terminally non-dropping, so $n=m, \pi_{\infty}$ is a $\mathrm{u}$ - $m$-embedding and $\pi_{\infty} \circ i^{\mathcal{T}}=i^{\mathcal{X}}$. 


\section{Minimal inflation, genericity inflation}

In this section we prove a comparison result for iteration trees, analogous to comparison of premice. The process we call minimal (simultaneous) inflation. We will need this result both in the construction of an iteration strategy for stacks of limit length, and in the extension of an iteration strategy with inflation condensation to a sufficiently small generic extension. We also introduce genericity inflation, an inflation analogue to genericity iteration.

\subsection{Minimal simultaneous inflation}

5.1 Definition. Let $\Omega>\omega$ be regular and let $\Sigma$ be a regularly $(\Omega+1)$-total conveniently inflationary strategy for $M$. Let $\mathscr{T}$ be a set of trees according to $\Sigma$, such that there is no surjection $\mathscr{T} \rightarrow \Omega .{ }^{34}$ The minimal (simultaneous) inflation of $\mathscr{T}$ is the tree $\mathcal{X}$ on $M$ with the following properties:

$-\mathcal{X}$ is according to $\Sigma$ (hence $\operatorname{lh}(\mathcal{X}) \leq \Omega+1$ ),

$-\mathcal{X}$ is an inflation of each $\mathcal{T} \in \mathscr{T}$; we write $t^{\mathcal{T}}=t^{\mathcal{T} \rightsquigarrow \mathcal{X}}$, etc, for $\mathcal{T} \in \mathscr{T}$,

- for each $\alpha+1<\operatorname{lh}(\mathcal{X})$ there is $\mathcal{T} \in \mathscr{T}$ such that $t^{\mathcal{T}}(\alpha)=0$,

- $\mathcal{X}$ has successor length,

- if $\operatorname{lh}(\mathcal{X})=\alpha+1<\Omega$ then for every $\mathcal{T} \in \mathscr{T}$, we have $\alpha \notin\left(C^{-}\right)^{\mathcal{T}} . \quad \dashv$

5.2 Lemma (Minimal simultaneous inflation). Let $\Omega, \mathscr{T}, \Sigma$ be as in 5.1. Then there is a unique minimal inflation $\mathcal{X}$ of $\mathscr{T}$. Moreover, there is $\mathcal{T} \in \mathscr{T}$ such that, with $\mathcal{T}^{\prime}=\operatorname{complete}^{\Sigma}(\mathcal{T})$, we have

- $\mathcal{X}$ is $\mathcal{T}^{\prime}$-terminally-non-dropping, and

- if $\operatorname{lh}(\mathcal{X})=\Omega+1$ then $\operatorname{lh}\left(\mathcal{T}^{\prime}\right)=\Omega+1$

Proof. We first verify uniqueness. Given $\alpha<\operatorname{lh}(\mathcal{X}) \cap \Omega$, we have $\alpha+1<\operatorname{lh}(\mathcal{X})$ iff $\alpha \in\left(C^{-}\right)^{\mathcal{T}}$ for some $\mathcal{T} \in \mathscr{T}$. And if $\alpha+1<\operatorname{lh}(\mathcal{X})$ then

$$
\operatorname{ind}\left(E_{\alpha}^{\mathcal{X}}\right)=\min \left(\left\{\operatorname{ind}\left(E_{\alpha}^{\mathcal{T} \rightsquigarrow \mathcal{X}}\right) \mid \alpha \in\left(C^{-}\right)^{\mathcal{T}}\right\}\right)
$$

as $\mathcal{X}$ is an inflation of every $\mathcal{T} \in \mathscr{T}$. So there is no freedom in the choice of extenders, and since $\mathcal{X}$ is via $\Sigma, \mathcal{X}$ is therefore unique.

Existence is by the proof of uniqueness and because inflations can be freely extended (as $\Sigma$ has inflation condensation).

We now verify the "moreover" clause.

Suppose first that $\ln (\mathcal{X})=\Omega+1$. For every $\beta$ such that $\beta+1<^{\mathcal{X}} \Omega$, there is $\mathcal{T} \in \mathscr{T}$ such that $t^{\mathcal{T}}(\beta)=0$. Since there is no surjection $\operatorname{card}(\mathscr{T}) \rightarrow \Omega$ and $\Omega$ is regular, we may fix $\mathcal{T} \in \mathscr{T}$ such that $t^{\mathcal{T}}(\beta)=0$ for cofinally many $\beta+1<^{\mathcal{X}} \Omega$.

\footnotetext{
${ }^{34}$ Since $\Omega$ is regular, it follows that there is no cofinal map $\mathscr{T} \rightarrow \Omega$. Note there is no restriction on $\operatorname{card}(M)$, but $\operatorname{lh}(\mathcal{T}) \leq \Omega+1$ for each $\mathcal{T} \in \mathscr{T}$.
} 
Let $\mathcal{T}^{\prime}=\operatorname{complete}^{\Sigma}(\mathcal{T})$. It follows that $\Omega \in C^{\mathcal{T}^{\prime}}$, and in fact, $\Omega=f^{\mathcal{T}^{\prime}}(\Omega)$ and $\Omega=\gamma_{\Omega}^{\mathcal{T}^{\prime}}$, so $\mathcal{X}$ is $\mathcal{T}^{\prime}$-terminally-non-dropping. ${ }^{35}$

Next suppose $\operatorname{lh}(\mathcal{X})=\beta+2=\alpha+1$ for some $\beta$. Then letting $\mathcal{T} \in \mathscr{T}$ be such that $t^{\mathcal{T}}(\beta)=0$, we have $\alpha=\beta+1 \in C^{\mathcal{T}}$. Since $\alpha \notin\left(C^{-}\right)^{\mathcal{T}}$, it follows that $\mathcal{T}$ has successor length and $\mathcal{X}$ is $\mathcal{T}$-terminally-non-dropping.

Finally suppose that $\ln (\mathcal{X})=\alpha+1<\Omega$ and $\alpha$ is a limit. Let $\beta<^{\mathcal{X}} \alpha$ be such that $(\beta, \alpha)_{\mathcal{X}} \cap \mathscr{D}_{\operatorname{deg}}^{\mathcal{X}}=\emptyset$. Fix $\mathcal{T} \in \mathscr{T}$ such that $t^{\mathcal{T}}(\beta)=0$, so $\beta \in C^{\mathcal{T}}$. Let $\mathcal{T}^{\prime}=\operatorname{complete}^{\Sigma}(\mathcal{T})$, so $\mathcal{X}$ is also an inflation of $\mathcal{T}^{\prime}$. Moreover, $\alpha \in C^{\mathcal{T}^{\prime} \rightsquigarrow \mathcal{X}}$ because $(\beta, \alpha)_{\mathcal{X}} \cap \mathscr{D}^{\mathcal{X}}=\emptyset$. But then $f^{\mathcal{T}^{\prime}}(\alpha)+1=\operatorname{lh}\left(\mathcal{T}^{\prime}\right)$, since $\alpha \notin\left(C^{-}\right)^{\mathcal{T}}$. Since also $(\beta, \alpha)_{\mathcal{X}}$ does not drop in model or degree and $f^{\mathcal{T}^{\prime}}(\beta)+1<\ln \left(\mathcal{T}^{\prime}\right)$, it follows that $\mathcal{X}$ is $\mathcal{T}^{\prime}$-terminally-non-dropping, as required.

\subsection{Genericity inflation}

Like for comparison, there is also an inflation analogue of genericity iteration, which we describe next. We won't actually use the technique in this paper, but it is easy to describe and worth noting, and the author has used it in other unpublished work, for the purposes mentioned in 5.7 below. ${ }^{36}$ Analogous results hold for slightly coherent wcpms and fine mice of both indexings (paired with their standard iteration rules). We first give the full proof for $\mathrm{u}-m$-sound seg-pms with MS-indexing (with MS-iteration rules). The proof adapts easily to the wcpm version, and we leave this to the reader; slight coherence ensures that the tree produced is normal. We will then explain how genericity iteration works for $\lambda$-indexed mice with $\lambda$-iteration rules, and finally sketch genericity inflation for this case. We state the results for the $\delta$-generator extender algebra, but the versions for the $\omega$-generator extender algebra are an easy corollary.

5.3 Definition. We write $\mathbb{B}_{\delta}$ for the $\delta$-generator extender algebra at $\delta$. When working inside a seg-pm or wcpm $M$, we only use extenders $E \in \mathbb{E}^{M}$ such that $\nu_{E}$ is an $M$-cardinal to induce extender algebra axioms (one can also require that $\nu_{E}$ is inaccessible in $M$, etc, as desired). Let $\kappa=\operatorname{cr}(E)$. Recall here that the axioms have the form

$$
\bigvee_{\alpha<\nu_{E}} \varphi_{\alpha} \Longrightarrow \bigvee_{\alpha<\kappa} \varphi_{\alpha}
$$

where $\vec{\varphi}=\left\langle\varphi_{\alpha}\right\rangle_{\alpha<\nu_{E}} \in M, \varphi_{\alpha} \in M \mid \kappa$ for all $\alpha<\kappa, \varphi_{\alpha} \in M \mid \nu_{E}$ for all $\alpha<\nu_{E}$, and $\vec{\varphi}=i_{E}^{M}(\vec{\varphi})\left\lceil\nu_{E}\right.$. (So $\vec{\varphi} \in \operatorname{Ult}(M, E)$, so $\vec{\varphi} \in M \mid \operatorname{ind}(E)$.) We use this definition independent of indexing.

Given an extender $G$ and $A \subseteq \mathrm{OR}$, we say that $G$ is $A$-bad iff $G$ induces a $\delta$ generator extender algebra axiom not satisfied by $A$ (equivalently, by $A \cap \nu_{G}$ ). $\quad \dashv$

5.4 Definition (Genericity inflation for MS-indexing and slightly coherent wcpms). Let $\Omega$ be regular uncountable. Let $M, \Sigma$ be such that either:

\footnotetext{
${ }^{35}$ Clearly this reflection argument uses only the regularity of $\Omega$, no AC.

${ }^{36}$ This technique and its application to self-iterability of mice was the author's first main motivation for considering inflation.
} 
- $M$ is a u- $m$-sound MS-indexed seg-pm and $\Sigma$ is a conveniently inflationary (u- $m, \Omega+1)$-strategy for $M$, or

- $M$ is a slightly coherent wcpm and $\Sigma$ is an inflationary $(\Omega+1)$-strategy for $M$,

and suppose that $\operatorname{card}(M)<\Omega$ (here if $M$ is a wcpm, which might not satisfy AC, we mean that $M$ is coded by some set $X \subseteq \eta<\Omega$ ). Let $\mathcal{T}$ be according to $\Sigma$, of limit length, and $\mathcal{T}^{\prime}=\mathcal{T}^{\wedge} \Sigma(\mathcal{T})$. Let $A \subseteq \Omega$. The $A$-genericity inflation of $\mathcal{T}$ is the tree $\mathcal{X}$ such that:

$-\mathcal{X}$ is a $\mathcal{T}^{\prime}$-terminally-non-dropping inflation of $\mathcal{T}^{\prime}$ (hence of successor length), according to $\Sigma$; write $C^{\mathcal{T}^{\prime}}=C^{\mathcal{T}^{\prime} \rightsquigarrow \mathcal{X}}$, etc.

- For every $\alpha+1<\operatorname{lh}(\mathcal{X})$, we have $\alpha \in\left(C^{-}\right)^{\mathcal{T}^{\prime}}$, and letting $\xi=\operatorname{ind}\left(E_{\alpha}^{\mathcal{T}^{\prime} \rightsquigarrow \mathcal{X}}\right)$, then $\operatorname{ind}\left(E_{\alpha}^{\mathcal{X}}\right)$ is the least $\gamma$ such that either $\gamma=\xi$, or:

- $F={ }_{\operatorname{def}} \mathbb{E}_{\gamma}\left(M_{\alpha}^{\mathcal{X}}\right)$ is $A$-bad, and

- if $M$ is MS-indexed then $\nu_{F}$ is a cardinal of $M_{\alpha}^{\mathcal{X}} \mid \xi$ (hence $F$ is total over $\left.M_{\alpha}^{\mathcal{X}} \mid \xi\right)$.

5.5 Remark. Note that if $\mathcal{X}$ is the $A$-genericity inflation of $\mathcal{T}$ and $\ln (\mathcal{X})=\alpha+1$, then $\alpha$ is least such that $f^{\mathcal{T} \rightsquigarrow \mathcal{X}}(\alpha)=\operatorname{lh}(\mathcal{T})=\operatorname{lh}\left(\mathcal{T}^{\prime}\right)-1$. So $\Sigma(\mathcal{T})$ is not relevant to the construction of $\mathcal{X}$; we need only $M, \Sigma, \mathcal{T}, A$. But $\mathcal{X}$ determines $\Sigma(\mathcal{T})$, hence $\mathcal{T}^{\prime}$, by inflation condensation.

5.6 Theorem. Let $\Omega, \Sigma, \mathcal{T}, A$ be as in 5.4. Then there is a unique $A$-genericity inflation $\mathcal{X}$ of $\mathcal{T}$ via $\Sigma$, and $\ln (\mathcal{X})=\Omega+1$ iff $\ln (\mathcal{T})=\Omega+1$.

Proof. The choice of extenders in $\mathcal{X}$ is clearly uniqued. The minimality of $\operatorname{lh}(\mathcal{X})$ and the requirement that it be via $\Sigma$, therefore determines $\mathcal{X}$ uniquely.

Now consider existence. Let us first verify that given a segment $\mathcal{X} \uparrow(\varepsilon+1)$ which is normal and such that $\mathcal{X}\lceil\varepsilon$ satisfies the properties stated above, then either $\varepsilon \in\left(C^{-}\right)^{\mathcal{T}^{\prime}}$ or $\mathcal{X}=\mathcal{X}\lceil(\varepsilon+1)$ is as desired. If $\varepsilon=0$ this is trivial and if $\varepsilon$ is a limit it holds by induction (if $\varepsilon \in C^{\mathcal{T}^{\prime}} \backslash\left(C^{-}\right)^{\mathcal{T}^{\prime}}$ then we are finished). If $M$ is a wcpm it is also automatic. So suppose $\varepsilon=\beta+1$ and $M$ is MS-indexed. We may assume that $t^{\mathcal{T}^{\prime}}(\beta)=1$. Let $\alpha=\operatorname{pred}^{\mathcal{X}}(\varepsilon+1)$. Then by induction, $\alpha \in\left(C^{-}\right)^{\mathcal{T}^{\prime}}$. We may also assume that $t^{\mathcal{T}^{\prime}}(\alpha)=1$. Then $\operatorname{cr}\left(E_{\beta}^{\mathcal{X}}\right)<\nu\left(E_{\alpha}^{\mathcal{X}}\right)$ and $E_{\beta}^{\mathcal{X}}$ is total over $M_{\alpha}^{\mathcal{X}} \mid \nu\left(E_{\alpha}^{\mathcal{X}}\right)$, but then $E_{\beta}^{\mathcal{X}}$ is total over

$$
K={ }_{\operatorname{def}} M_{\alpha}^{\mathcal{X}} \mid \operatorname{ind}\left(E_{\alpha}^{\mathcal{T}^{\prime} \rightsquigarrow \mathcal{X}}\right),
$$

because, by construction, $\nu\left(E_{\alpha}^{\mathcal{X}}\right)$ is a cardinal of $K$. So $\varepsilon \in\left(C^{-}\right)^{\mathcal{T}^{\prime}}$ as required.

Now $\mathcal{X}$ is monotone index-increasing; that is, if $\alpha+1<\beta+1<\operatorname{lh}(\mathcal{X})$ then $\operatorname{ind}\left(E_{\alpha}^{\mathcal{X}}\right) \leq \operatorname{ind}\left(E_{\beta}^{\mathcal{X}}\right)$. For suppose not and let $(\alpha, \beta)$ be least such. Suppose $M$ is MS-indexed. Since $\mathcal{X} \uparrow(\beta+1)$ is an inflation of $\mathcal{T}$,

$$
\xi={ }_{\operatorname{def}} \operatorname{ind}\left(E_{\beta}^{\mathcal{T} \rightsquigarrow \mathcal{X}}\right) \geq \operatorname{ind}\left(E_{\alpha}^{\mathcal{X}}\right) .
$$


Since $\operatorname{ind}\left(E_{\beta}^{\mathcal{X}}\right)<\operatorname{ind}\left(E_{\alpha}^{\mathcal{X}}\right)$, note $E_{\beta}^{\mathcal{X}}$ is $A$-bad and $\nu\left(E_{\beta}^{\mathcal{X}}\right) \leq \widetilde{\nu}\left(E_{\alpha}^{\mathcal{X}}\right)<\operatorname{ind}\left(E_{\alpha}^{\mathcal{X}}\right)$ and $\nu\left(E_{\beta}^{\mathcal{X}}\right)$ is a cardinal of $M_{\beta}^{\mathcal{X}} \mid \xi$. But then by coherence, $E_{\beta}^{\mathcal{X}} \in \mathbb{E}\left(M_{\alpha}^{\mathcal{X}}\right)$ and $\nu\left(E_{\beta}^{\mathcal{X}}\right)$ is a cardinal of $M_{\alpha}^{\mathcal{X}} \mid \operatorname{ind}\left(E_{\alpha}^{\mathcal{X}}\right)$, which implies that we should have used $E_{\beta}^{\mathcal{X}}$ at stage $\alpha$, contradiction. If instead $M$ is a wcpm then one uses slight coherence for a similar argument.

It remains to see that if we reach $\mathcal{X}$ of length $\Omega+1$, then $\Omega+1=\ln \left(\mathcal{T}^{\prime}\right)$ and $\Omega=f^{\mathcal{T}^{\prime}}(\Omega)$. We may assume ZFC, by noting that the entire construction takes place in $L[X, \Sigma, \mathcal{T}, A]$ where $X \subseteq \eta<\Omega$ codes $M$. We have $\Omega \in C^{\mathcal{T}^{\prime}}$, and moreover, it suffices to see that $t^{\mathcal{T}^{\prime}}(\beta)=0$ for cofinally many $\beta+1<\mathcal{X} \Omega$. Let $\eta$ be large and $\pi: H \rightarrow V_{\eta}$ be elementary with $\pi(\mu)=\Omega$ where $\operatorname{cr}(\pi)=$ $\mu$, and everything relevant in $\operatorname{rg}(\pi)$. Then by the usual calculations, letting $\beta+1=\operatorname{succ}^{\mathcal{X}}(\mu, \Omega), E_{\beta}^{\mathcal{X}}$ coheres $A$ through $\nu\left(E_{\beta}^{\mathcal{X}}\right)$, and hence, $E_{\beta}^{\mathcal{X}}$ is not $A$-bad. Therefore $t^{\mathcal{T}^{\prime}}(\beta)=0$. So by elementarity, we are done.

5.7 Remark. Note that to construct $\mathcal{X}$, the information we actually need is $M, \mathcal{T}, A$, and the sequence of branches actually used in forming $\mathcal{X}$. Moreover, from $\mathcal{X}$ we can compute $\Sigma(\mathcal{T})$. Thus, genericity inflations (and variants thereof) provide a natural method to attempt to compute $\Sigma(\mathcal{T})$, if we know how to compute $\Sigma(\mathcal{X})$ for enough trees $\mathcal{X}$ : one builds such an $\mathcal{X}$ into which $\mathcal{T}$ is embedded. An application of this is some unpublished work of the author's, showing that a (in the more interesting case, non-tame) premouse $M$ computes some fragment of its own iteration strategy; an instance of this method will also be used in [7]. This application incorporates and generalizes the methods of [10], which covers a large part of the technical issues, but is limited to tame mice. In this context, $\mathcal{T}$ is some tree on $M$ or a segment thereof, $\mathcal{T} \in M$, and $A=\mathbb{E}^{M}$. One uses P-constructions/*-translations to compute $\Sigma(\mathcal{X}\lceil\eta)$ for limits $\eta$ (see [1], [10], augmented with [15]). Note that because the computation of $C^{\mathcal{T}}$ and $E_{\alpha}^{\mathcal{T} \leadsto \mathcal{X}}$ is local, at non-trivial limit stages $\eta$ of the genericity inflation, with $\delta=\delta(\mathcal{X}\lceil\eta)$, we get that $\mathcal{X}\lceil\eta$ is definable from parameters over $M \mid \delta$ (to arrange this, one might need to insert short linear iterations into the genericity inflation, to ensure that the $*$-translations of the Q-structures determining earlier branch choices are proper segments of $M \mid \delta$; such arguments appear in [16]). Because we have also made $M \mid \delta$ generic, we have the necessary base for forming Pconstructions/*-translations. For example, we might want to use this method to prove that $M \models$ "My countable proper segments are $\left(\omega, \omega_{1}\right)$-iterable" (maybe above some $\left.\alpha<\omega_{1}^{M}\right)$. For arbitrary non-tame mice, there seem to be subtleties in proving that the genericity inflation process terminates prior to $\omega_{1}^{M}$ in $M$. But in typical " $\varphi$-minimal" mice (for example, the sharp for the least proper class mouse satisfying "There is a superstrong extender"), it does.

We now discuss the version for $\lambda$-indexing and $\lambda$-iteration rules. We first describe how standard genericity iteration works for $\lambda$-indexed mice with $\lambda$ iteration rules. ${ }^{37}$ The main difference between this and standard genericity iteration (for MS-indexing with MS-iteration rules) is that we will allow drops

\footnotetext{
${ }^{37}$ The methods here are related to those used by the author in [11] to translate between different iteration rules for $\lambda$-indexed mice.
} 
in model to appear at intermediate stages of the iteration. We will thus need to be a little careful to ensure that the eventual main branch is non-dropping. In our original attempted proof, we had ignored the fact that the collection of extenders used to induce extender algebra axioms are not cohered by extenders $E$ through $\lambda(E)$. We thank Stefan Miedzianowski for pointing this issue out. Fortunately a fix was available for this problem.

5.8 Theorem (Genericity iteration for $\lambda$-indexing). Let $\Omega>\omega$ be regular. Let $M$ be a $\lambda$-indexed pm with $\operatorname{card}(M)<\Omega$. Let $\Sigma$ be a $(0, \Omega+1)$-strategy for $M$ (for $\lambda$-iteration rules). Let $\delta \in \mathrm{OR}^{M}$ be such that $M \models " \delta$ is Woodin as witnessed by $\mathbb{E} "$. Let $A \subseteq \Omega$.

Then there is $\mathcal{T}$ on $M$ via $\Sigma$, of length $\alpha+1<\Omega$, such that $[0, \alpha)_{\mathcal{T}}$ does not drop in model, and $A \cap \delta^{\prime}$ is $M_{\alpha}^{\mathcal{T}}$-generic for $\mathbb{B}_{\delta^{\prime}}\left(M_{\alpha}^{\mathcal{T}}\right)$, where $\delta^{\prime}=i_{0 \alpha}^{\mathcal{T}}(\delta)$.

Proof. We form $\mathcal{T}$ as follows. Suppose we have defined $\mathcal{T} \uparrow(\alpha+1)$, but it doesn't yet witness the theorem. We (attempt to) define a sequence $\left\langle M_{\alpha i}\right\rangle_{i \leq k_{\alpha}}$, with $k_{\alpha}<\omega$, and with $M_{\alpha i}$ an active segment of $M_{\alpha}$ and $M_{\alpha, i+1} \triangleleft M_{\alpha i}$. Let $M_{\alpha 0}$, if it exists, be the least $N \unlhd M_{\alpha}$ such that $N$ is active and letting $G=F^{N}$, either

$-[0, \alpha]_{\mathcal{T}}$ drops in model and $N=M_{\alpha}$, or

- $\nu_{G}$ is a cardinal ${ }^{38}$ of $M_{\alpha}, G$ is $A$-bad ${ }^{39}$ and if $[0, \alpha]_{\mathcal{T}}$ does not drop in model then $\operatorname{ind}(G)<i_{0 \alpha}^{\mathcal{T}}(\delta)$.

If $M_{\alpha 0}$ does not exist then we terminate the process, setting $\mathcal{T}=\mathcal{T}\lceil\alpha+1$.

Suppose that $M_{\alpha i}$ exists where $i<\omega$. Then $M_{\alpha, i+1}$, if it exists, is the least $N \triangleleft M_{\alpha i}$ such that $N$ is active with $G=F^{N}, \nu_{G}$ is a cardinal of $M_{\alpha i}$ and $G$ is $A$-bad. If $M_{\alpha, i+1}$ does not exist then set $k_{\alpha}=i$ and $E_{\alpha}^{\mathcal{T}}=F\left(M_{\alpha k_{\alpha}}\right)$.

We claim that this works. Suppose not. For each $\alpha+1<\operatorname{lh}(\mathcal{T})$ (hence, $M_{\alpha 0}$ exists) let $\nu_{\alpha i}=\nu\left(F\left(M_{\alpha i}\right)\right)$.

Claim 1. Let $\alpha+1<\operatorname{lh}(\mathcal{T})$ with $M_{\alpha 0}=M_{\alpha}$. Then $k_{\alpha}=0$, so $E_{\alpha}^{\mathcal{T}}=F^{M_{\alpha}}$.

Proof. Otherwise $M_{\alpha 1}$ would contradict the minimality of the choice of $M_{\alpha 0}$.

Claim 2. Let $\alpha+1<\operatorname{lh}(\mathcal{T})$ with $M_{\alpha 0} \triangleleft M_{\alpha}$. Then:

1. $\rho_{1}\left(M_{\alpha i}\right)=\rho_{\omega}\left(M_{\alpha i}\right)=\nu_{\alpha i}$.

2. $\nu_{\alpha 0}<\ldots<\nu_{\alpha k_{\alpha}}$.

3. The reverse model dropdown sequence of $\left(M_{\alpha}\right.$, ind $\left.\left(E_{\alpha}^{\mathcal{T}}\right)\right)$ is $\left\langle M_{\alpha i}\right\rangle_{i \leq k_{\alpha}}$.

Proof. Part 1: For any active premouse $N, \rho_{1}^{N} \leq \nu\left(F^{N}\right)$. But $\rho_{\omega}\left(M_{\alpha i}\right) \geq \nu_{\alpha i}$, because either:

$-i=0$ and $\nu_{\alpha 0}$ is a cardinal of $M_{\alpha}$ and $M_{\alpha 0} \triangleleft M_{\alpha}$, or

\footnotetext{
${ }^{38}$ If one only forms extender algebra axioms with extenders $E$ with $\nu_{E}$ inaccessible, then one could also assume here that $\nu_{G}$ is inaccessible in $M_{\alpha}^{\mathcal{T}}$.

${ }^{39}$ This makes sense even if $[0, \alpha]_{\mathcal{T}}$ drops, as the requirements are local.
} 
- $i>0$ and $\nu_{\alpha i}$ is a cardinal of $M_{\alpha, i-1}$ and $M_{\alpha i} \triangleleft M_{\alpha, i-1}$.

Part 2: Suppose $\nu_{\alpha, i+1} \leq \nu_{\alpha i}$. Then we contradict the minimality of $M_{\alpha i}$. That is, if $i=0$, then $\nu_{\alpha, i+1}$ is a cardinal of $M_{\alpha}$, but $M_{\alpha, i+1} \triangleleft M_{\alpha i} \unlhd M_{\alpha}$, so we should have chosen $M_{\alpha, i+1}$ over $M_{\alpha i}$. It is similar if $i>0$.

Part 3: Because $\nu_{\alpha, i+1}$ is a cardinal in $M_{\alpha i}$, and $\nu_{\alpha 0}$ a cardinal in $M_{\alpha}$, this follows from the previous parts.

Claim 3. Let $\beta<\operatorname{lh}(\mathcal{T})$ be such that $[0, \beta]_{\mathcal{T}}$ drops in model. Then $M_{\beta}$ is active. Moreover, let $\gamma+1 \leq^{\mathcal{T}} \beta$ be such that $\gamma+1 \in \mathscr{D}^{\mathcal{T}}$ and $(\gamma+1, \beta]_{\mathcal{T}}$ does not drop in model, and let $\alpha=\operatorname{pred}^{\mathcal{T}}(\gamma+1)$. Then $M_{\alpha 0} \triangleleft M_{\alpha}$ and $M_{\gamma+1}^{* \mathcal{T}}=M_{\alpha i}$ for some $i \leq k_{\alpha}$, and $F^{M_{\beta}}\left\lceil\nu_{\alpha i}=F\left(M_{\alpha i}\right)\left\lceil\nu_{\alpha i}\right.\right.$.

Proof. Because $\gamma+1 \in \mathscr{D}^{\mathcal{T}}, M_{\gamma+1}^{*}$ is in the $\left(M_{\alpha}, \operatorname{ind}\left(E_{\alpha}\right)\right)$-dropdown, so by Claims 1 and 2, $M_{\alpha 0} \triangleleft M_{\alpha}$ and $M_{\gamma+1}^{*}=M_{\alpha i}$ for some $i$. Therefore $M_{\beta}$ is active. But also by Claim 2, $\nu_{\alpha i}=\rho_{\omega}\left(M_{\alpha i}\right) \leq \operatorname{cr}\left(E_{\gamma}\right)$, and so $F^{M_{\beta}}\left\lceil\nu_{\alpha i} \subseteq F\left(M_{\alpha i}\right)\right.$.

Claim 4. $\mathcal{T}$ is normal.

Proof. We just need to see that $\operatorname{ind}\left(E_{\alpha}\right)<\operatorname{ind}\left(E_{\beta}\right)$ for $\alpha<\beta$. But otherwise, letting $(\alpha, \beta)$ be the least counterexample, then since $\left(\mathrm{ex}_{\alpha}^{\mathcal{T}}\right)^{\mathrm{pv}}=\left(M_{\alpha k_{\alpha}}\right)^{\mathrm{pv}}$ is a cardinal segment of $M_{\beta}$, we easily get that $M_{\beta 0} \triangleleft M_{\alpha k_{\alpha}}$, and reach a contradiction to the maximality of $k_{\alpha}$ (that is, $M_{\alpha, k_{\alpha}+1}$ exists, a contradiction).

By Claim 3 and by construction, if $\mathcal{T}$ terminates in length $\alpha+1<\Omega$, then $[0, \alpha]_{\mathcal{T}}$ does not drop, so we are done. So it suffices to prove:

Claim 5. $\mathcal{T}$ terminates with length $<\Omega$.

Proof. We may assume ZFC, by working in $L[M, \Sigma, A]$, where we have $\mathcal{T}$. Suppose that we reach $\mathcal{T}$ of length $\Omega+1$. Let $\pi: N \rightarrow V_{\eta}$ be elementary, where $\eta$ is large and $N$ is transitive with $\operatorname{card}(N)<\Omega, \operatorname{cr}(\pi)=\kappa$ and $\pi(\kappa)=\Omega$, and the relevant objects are in $\operatorname{rg}(\pi)$. Then as usual, $M_{\kappa}^{\mathcal{T}} \in N, i_{\kappa \Omega}^{\mathcal{T}} \subseteq \pi$, and $\pi(A \cap \kappa)=A$. Let $\beta+1<^{\mathcal{T}} \Omega$ with $\operatorname{pred}^{\mathcal{T}}(\beta+1)=\kappa$. By the usual argument that genericity iterations for MS-indexing terminate, $E_{\beta}$ is not $A$-bad, so $[0, \beta]_{\mathcal{T}}$ drops in model and $E_{\beta}=F\left(M_{\beta}\right)$. So by Claim 3 there is $\alpha<^{\mathcal{T}} \beta$ and $i \leq k_{\alpha}$ such that $F\left(M_{\alpha i}\right)$ is $A$-bad and $F\left(M_{\alpha i}\right)\left\lceil\nu_{\alpha i}=E_{\beta}\left\lceil\nu_{\alpha i}\right.\right.$. But then again, the usual argument gives a contradiction.

This completes the proof.

Finally, genericity inflation for $\lambda$-iteration rules is just a straightforward combination of the preceding methods:

5.9 Definition (Genericity inflation for $\lambda$-indexing). Let $\Omega>\omega$ be regular. Let $M$ be an $m$-sound $\lambda$-indexed premouse with $\operatorname{card}(M)<\Omega$. Let $\Sigma$ be an inflationary $(m, \Omega+1)$-strategy for $M$ (for $\lambda$-iteration rules). Let $\mathcal{T}$ be according to $\Sigma$, of limit length, and $\mathcal{T}^{\prime}=\mathcal{T}^{\wedge} \Sigma(\mathcal{T})$. Let $A \subseteq \Omega$. The $A$-genericity inflation of $\mathcal{T}$ is the tree $\mathcal{X}$ such that: 
- $\mathcal{X}$ is a $\mathcal{T}^{\prime}$-terminally-non-dropping inflation of $\mathcal{T}^{\prime}$ (hence of successor length), according to $\Sigma$; write $C^{\mathcal{T}^{\prime}}=C^{\mathcal{T}^{\prime} \rightsquigarrow \mathcal{X}}$, etc.

- If $\alpha+1 \leq \operatorname{lh}(\mathcal{X})$ and $\mathcal{X}\left\lceil\alpha+1\right.$ is $\mathcal{T}^{\prime}$-terminally-non-dropping then $\alpha+1=$ $\operatorname{lh}(\mathcal{X})$.

- Let $\alpha+1<\operatorname{lh}(\mathcal{X})$. We define $\xi_{\alpha}, k_{\alpha}<\omega,\left\langle M_{\alpha i}\right\rangle_{i \leq k_{\alpha}}$ and $E_{\alpha}^{\mathcal{X}}$ as follows:

- If $\alpha \in\left(C^{-}\right)^{\mathcal{T}^{\prime}}$ then $\xi_{\alpha}=\operatorname{ind}\left(E_{\alpha}^{\mathcal{T}^{\prime} \rightsquigarrow \mathcal{X}}\right)$.

- If $\alpha \notin\left(C^{-}\right)^{\mathcal{T}^{\prime}}$ then $\xi_{\alpha}=\operatorname{OR}\left(M_{\alpha}^{\mathcal{X}}\right)$; in this case, $[0, \alpha]_{\mathcal{X}}$ drops in model and $M_{\alpha}^{\mathcal{X}}$ is active.

- $M_{\alpha 0}$ is the least $N \unlhd M_{\alpha}^{\mathcal{X}} \mid \xi_{\alpha}$ such that either $N=M_{\alpha}^{\mathcal{X}} \mid \xi_{\alpha}$ or $N$ is active with $G=F^{N}, \nu_{G}$ is an $M_{\alpha}^{\mathcal{X}} \mid \xi_{\alpha}$-cardinal and $G$ is $A$-bad.

- $k_{\alpha}$ and $\left\langle M_{\alpha i}\right\rangle_{0<i \leq k_{\alpha}}$ are determined from $M_{\alpha 0}$ as in the proof of 5.8.

- $E_{\alpha}^{\mathcal{X}}=F\left(M_{\alpha k_{\alpha}}\right)$.

A straightforward combination of the proofs of 5.8 and 5.6 gives:

5.10 Theorem. Let $\Omega, \Sigma, \mathcal{T}, A$ be as in 5.9. Then there is a unique $A$-genericity inflation $\mathcal{X}$ of $\mathcal{T}$ via $\Sigma$, and $\ln (\mathcal{X})=\Omega+1$ iff $\ln (\mathcal{T})=\Omega+1$.

\section{Commutativity of inflation}

We will later show that a normal iteration strategy with inflation condensation induces a strategy for stacks $\overrightarrow{\mathcal{T}}$ of normal trees. The latter strategy will be such that we can embed the last model of $\overrightarrow{\mathcal{T}}$ into the last model of a normal tree $\mathcal{X}$. The tree $\mathcal{X}$ will be produced by inflation; for example, if $\overrightarrow{\mathcal{T}}=\left(\mathcal{T}_{0}, \mathcal{T}_{1}\right)$ where each $\mathcal{T}_{i}$ is normal, then $\mathcal{X}$ will be an inflation of $\mathcal{T}_{0}$. For infinite stacks, we will produce an infinite sequence of trees $\left\langle\mathcal{X}_{\alpha}\right\rangle_{\alpha<\eta}$, with $\mathcal{X}_{\beta}$ an inflation of $\mathcal{X}_{\alpha}$ for each $\alpha<\beta$. In this section we establish a key commutativity lemma which helps us understand this situation. We will also use the lemma in $\S 7$, when we extend an iteration strategy with inflation condensation to a sufficiently small generic extension. We state the coarse version of the lemma first, as it contains the main points, and then state and prove the fine version. A key point to note is that the commutativity lemmas hold for arbitrary trees and inflations (satisfying certain conditions); we do not assume that the trees are via a strategy with condensation.

6.1 Lemma (Commutativity of inflation (coarse)). Let $M$ be a wcpm and $\mathcal{X}_{0}$, $\mathcal{X}_{1}, \mathcal{X}_{2}$ be normal on $M, \mathcal{X}_{i+1}$ an inflation of $\mathcal{X}_{i}$, with $\mathcal{X}_{1}$ being non- $\mathcal{X}_{0}$-pending (but $\mathcal{X}_{2}$ could be $\mathcal{X}_{1}$-pending). Then $\mathcal{X}_{2}$ is an inflation of $\mathcal{X}_{0}$, and things commute in a reasonable fashion. That is, let

$$
\left(t^{i j}, C^{i j},\left(C^{-}\right)^{i j}, f^{i j},\left\langle\Pi_{\alpha}^{i j}\right\rangle_{\alpha \in C^{i j}}\right)=(t, C, \ldots)^{\mathcal{X}_{i} \rightsquigarrow \mathcal{X}_{j}}
$$

for $i<j$; we also use analogous notation for other associated objects. (Note that $C^{i j}=\ln \left(\mathcal{X}_{j}\right)$ for each $i, j$, because $M$ is a wcpm.) Let $\alpha_{2}<\ln \left(\mathcal{X}_{2}\right)$ and $\alpha_{k}=f^{k 2}\left(\alpha_{2}\right)$. Then (cf. Figure 5): 

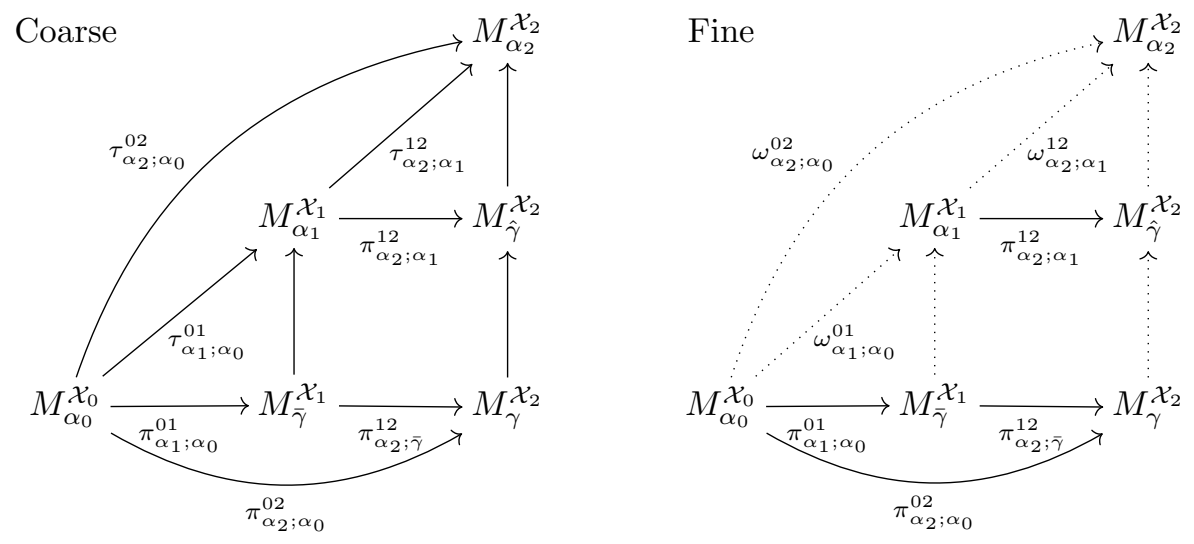

Figure 5: Commutativity of inflation, coarse and fine. In both diagrams, $\alpha_{2} \in$ $C^{02}, \alpha_{1}=f^{12}\left(\alpha_{2}\right), \alpha_{0}=f^{02}\left(\alpha_{2}\right)=f^{01}\left(\alpha_{1}\right), \bar{\gamma}=\gamma_{\alpha_{1} ; \alpha_{0}}^{01}, \gamma=\gamma_{\alpha_{2} ; \alpha_{0}}^{02}=\gamma_{\alpha_{2} ; \bar{\gamma}}^{12}$ and $\hat{\gamma}=\gamma_{\alpha_{2} ; \alpha_{1}}^{12}$. Note $\alpha_{2}=\delta_{\alpha_{2} ; \alpha_{0}}^{02}=\delta_{\alpha_{2} ; \alpha_{1}}^{12}$ and $\alpha_{1}=\delta_{\alpha_{1} ; \alpha_{0}}^{01}$ and $\bar{\gamma} \leq^{\mathcal{X}_{1}} \alpha_{1}$ and $\gamma \leq^{\mathcal{X}_{2}}$ $\hat{\gamma} \leq{ }^{\mathcal{X}_{2}} \alpha_{2}$. Solid arrows indicate total embeddings, and dotted arrows indicate partial embeddings (the domain and codomain are initial segments of the models in the figure). The vertical arrows are (partial) iteration embeddings. Both diagrams commute, after restricting to common domains in the fine diagram. For example, $\operatorname{dom}\left(\omega_{\alpha_{2} ; \alpha_{1}}^{12} \circ \omega_{\alpha_{1} ; \alpha_{0}}^{01}\right) \subseteq \operatorname{dom}\left(\omega_{\alpha_{2} ; \alpha_{0}}^{02}\right)$ and these maps agree over the smaller domain. Note that in the fine diagram, while the maps $\omega_{\alpha_{\ell} ; \alpha_{k}}^{k \ell}$ are the only ones displayed mapping directly between segments of $M_{\alpha_{k}}^{\mathcal{X}_{k}}$ and $M_{\alpha_{\ell}}^{\mathcal{X}_{\ell}}$, there could be maps $\tau_{\alpha_{\ell} ; \alpha_{k} i}^{k \ell}$ mapping between larger segments thereof, and these also commute with the rest of the diagram. 
1. $\alpha_{0}=f^{02}\left(\alpha_{2}\right)=f^{01}\left(f^{12}\left(\alpha_{2}\right)\right)=f^{01}\left(\alpha_{1}\right)$.

2. Suppose $\alpha_{2}+1<\operatorname{lh}\left(\mathcal{X}_{2}\right)$ and let $E_{2}=E_{\alpha_{2}}^{\mathcal{X}_{2}}$. Then:

- $E_{2}$ is the $\mathcal{X}_{0} \rightsquigarrow \mathcal{X}_{2}$-copy of an extender $E_{0}\left(\right.$ so $\left.E_{0}=E_{\alpha_{0}}^{\mathcal{X}_{0}}\right)$

iff

- $E_{2}$ is the $\mathcal{X}_{1} \rightsquigarrow \mathcal{X}_{2}$-copy of an extender $E_{1}\left(\right.$ so $\left.E_{1}=E_{\alpha_{1}}^{\mathcal{X}_{1}}\right)$ and

- $E_{1}$ is the $\mathcal{X}_{0} \rightsquigarrow \mathcal{X}_{1}$-copy of $E_{0}$.

That is, $\alpha_{2} \in\left(C^{-}\right)^{02}$ and $t^{02}\left(\alpha_{2}\right)=0$ iff

$$
\alpha_{2} \in\left(C^{-}\right)^{12} \text { and } t^{12}\left(\alpha_{2}\right)=0 \text { and } \alpha_{1} \in\left(C^{-}\right)^{01} \text { and } t^{01}\left(\alpha_{1}\right)=0 .
$$

4. We have:

(a) If $\beta \leq \alpha_{0}$ and $\gamma=\gamma_{\alpha_{1} ; \beta}^{01}$ then $\gamma_{\alpha_{2} ; \beta}^{02}=\gamma_{\alpha_{2} ; \gamma}^{12}$ and $\pi_{\alpha_{2} ; \beta}^{02}=\pi_{\alpha_{2} ; \gamma}^{12} \circ \pi_{\alpha_{1} ; \beta}^{01}$.

(b) $\bigcup_{\beta \leq \alpha_{0}} I_{\alpha_{2} ; \beta}^{02} \subseteq \bigcup_{\beta \leq \alpha_{1}} I_{\alpha_{2} ; \beta}^{12}$.

(c) If $\beta \leq \alpha_{0}$ and $\gamma \in I_{\alpha_{2} ; \beta}^{02}$ then $f^{12}(\gamma) \in I_{\alpha_{1} ; \beta}^{01}$.

5. Let $\gamma^{02}=\gamma_{\alpha_{2} ; \alpha_{0}}^{02}$ and $\gamma^{01}=\gamma_{\alpha_{1} ; \alpha_{0}}^{01}$ and $\gamma^{12}=\gamma_{\alpha_{2} ; \alpha_{1}}^{12}\left(\right.$ maybe $\left.\gamma^{12} \neq \gamma_{\alpha_{2} ; \gamma^{01}}^{12}\right)$. Note that for $k<\ell \leq 2$, we have

$$
\tau_{\alpha_{\ell} ; \alpha_{k}}^{k \ell}=j_{\gamma^{k \ell} \alpha_{\ell}}^{\mathcal{X}_{\ell}} \circ \pi_{\alpha_{\ell} ; \alpha_{k}}^{k \ell}: M_{\alpha_{k} i^{k \ell}}^{\mathcal{X}_{k}} \rightarrow M_{\alpha_{\ell}}^{\mathcal{X}_{\ell}} .
$$

Then $\tau_{\alpha_{2} ; \alpha_{0}}^{02}=\tau_{\alpha_{2} ; \alpha_{1}}^{12} \circ \tau_{\alpha_{1} ; \alpha_{0}}^{01}$. Therefore if $\operatorname{lh}\left(\mathcal{X}_{2}\right)=\alpha_{2}+1$ and $\operatorname{lh}\left(\mathcal{X}_{1}\right)=$ $\alpha_{1}+1$, then $\pi_{\infty}^{02}=\pi_{\infty}^{12} \circ \pi_{\infty}^{01}$.

6.2 Lemma (Commutativity of inflation (fine)). Let $M$ be u-m-sound, let $\mathcal{X}_{0}, \mathcal{X}_{1}, \mathcal{X}_{2}$ be u-m-maximal on $M, \mathcal{X}_{i+1}$ an inflation of $\mathcal{X}_{i}$, with $\mathcal{X}_{1}$ non- $\mathcal{X}_{0^{-}}$ pending (but $\mathcal{X}_{2}$ could be $\mathcal{X}_{1}$-pending). Then $\mathcal{X}_{2}$ is an inflation of $\mathcal{X}_{0}$, and things commute in a reasonable fashion. That is, let

$$
\left(t^{i j}, C^{i j},\left(C^{-}\right)^{i j}, f^{i j},\left\langle\Pi_{\alpha}^{i j}\right\rangle_{\alpha \in C^{i j}}\right)=(t, C, \ldots)^{\mathcal{X}_{i} \rightsquigarrow \mathcal{X}_{j}}
$$

for $i<j$; we also use analogous notation for other associated objects. Let $\alpha_{2}<\operatorname{lh}\left(\mathcal{X}_{2}\right)$. If $k<2$ and $\alpha_{2} \in C^{k 2}$ let $\alpha_{k}=f^{k 2}\left(\alpha_{2}\right)$. Then (cf. Figure 5, which depicts a key case of the lemma):

1. If $\alpha_{2} \in C^{02}$ then $\alpha_{2} \in C^{12}, \alpha_{1} \in C^{01}$ and

$$
\alpha_{0}=f^{02}\left(\alpha_{2}\right)=f^{01}\left(f^{12}\left(\alpha_{2}\right)\right)=f^{01}\left(\alpha_{1}\right) .
$$

2. Suppose $\alpha_{2}+1<\operatorname{lh}\left(\mathcal{X}_{2}\right)$ and let $E_{2}=E_{\alpha_{2}}^{\mathcal{X}_{2}}$. Then:

- $E_{2}$ is the $\mathcal{X}_{0} \rightsquigarrow \mathcal{X}_{2}$-copy of an extender $E_{0}\left(\right.$ so $\left.E_{0}=E_{\alpha_{0}}^{\mathcal{X}_{0}}\right)$

iff 
- $E_{2}$ is the $\mathcal{X}_{1} \rightsquigarrow \mathcal{X}_{2}$-copy of an extender $E_{1}$ (so $E_{1}=E_{\alpha_{1}}^{\mathcal{X}_{1}}$ ), and

- $E_{1}$ is the $\mathcal{X}_{0} \rightsquigarrow \mathcal{X}_{1}$-copy of $E_{0}$.

That is, $\alpha_{2} \in\left(C^{-}\right)^{02}$ and $t^{02}\left(\alpha_{2}\right)=0$ iff

$$
\alpha_{2} \in\left(C^{-}\right)^{12} \text { and } t^{12}\left(\alpha_{2}\right)=0 \text { and } \alpha_{1} \in\left(C^{-}\right)^{01} \text { and } t^{01}\left(\alpha_{1}\right)=0 .
$$

3. Suppose $\alpha_{2} \in C^{12}$ and $\alpha_{1} \in C^{01}{ }^{40}$ Then:

(a) If $\alpha_{1}+1=\ln \left(\mathcal{X}_{1}\right)$ then $\alpha_{2} \in C^{02}$.

(b) If $\beta \leq f^{01}\left(\alpha_{1}\right)$ and $\xi \in I_{\alpha_{1} ; \beta}^{01}$ then $\gamma_{\alpha_{2} ; \xi}^{12} \in C^{02}$.

(c) If $\beta<f^{01}\left(\alpha_{1}\right)$ and $\xi=\delta_{\alpha_{1} ; \beta}^{01}$ then $\delta_{\alpha_{2} ; \xi}^{12} \in C^{02}$.

4. Suppose $\alpha_{2} \in C^{02}$. Then:

(a) If $\beta \leq \alpha_{0}$ and $\gamma=\gamma_{\alpha_{1} ; \beta}^{01}$ then $\gamma_{\alpha_{2} ; \beta}^{02}=\gamma_{\alpha_{2} ; \gamma}^{12}$ and $\pi_{\alpha_{2} ; \beta}^{02}=\pi_{\alpha_{2} ; \gamma}^{12} \circ \pi_{\alpha_{1} ; \beta}^{01}$.

(b) $\bigcup_{\beta \leq \alpha_{0}} I_{\alpha_{2} ; \beta}^{02} \subseteq \bigcup_{\beta \leq \alpha_{1}} I_{\alpha_{2} ; \beta}^{12} \subseteq C^{12}$.

(c) If $\beta \leq \alpha_{0}$ and $\gamma \in I_{\alpha_{2} ; \beta}^{02}$ then $f^{12}(\gamma) \in I_{\alpha_{1} ; \beta}^{01}$.

5. Suppose $\alpha_{2} \in C^{02}$. Let $\gamma^{02}=\gamma_{\alpha_{2} ; \alpha_{0}}^{02}$ and $\gamma^{01}=\gamma_{\alpha_{1} ; \alpha_{0}}^{01}$ and $\gamma^{12}=\gamma_{\alpha_{2} ; \alpha_{1}}^{12}$ (maybe $\gamma^{12} \neq \gamma_{\alpha_{2} ; \gamma^{01}}^{12}$ ) and $i^{02}=i_{\alpha_{2} ; \alpha_{0}}^{02}$ and $i^{01}=i_{\alpha_{1} ; \alpha_{0}}^{01}$ and $i^{12}=i_{\alpha_{2} ; \alpha_{1}}^{12}$. Note that

$$
\tau_{\alpha_{\ell} ; \alpha_{k} i^{k \ell}}^{k \ell}=j_{\gamma^{k \ell}, \alpha_{\ell}}^{\mathcal{X}_{\ell}} \circ \pi_{\alpha_{\ell} ; \alpha_{k}}^{k \ell}\left\lceil M_{\alpha_{k} i^{k \ell}}^{\mathcal{X}_{k}}: M_{\alpha_{k} i^{k \ell}}^{\mathcal{X}_{k}} \rightarrow M_{\alpha_{\ell}}^{\mathcal{X}_{\ell}}\right.
$$

for $k<\ell \leq 2$. Then we have:

(a) $i^{01} \leq i^{02}\left(\right.$ so $M_{\alpha_{0} i^{02}}^{\mathcal{X}_{0}} \unlhd M_{\alpha_{0} i^{01}}^{\mathcal{X}_{0}}$, with equality iff $\left.i^{02}=i^{01}\right)$.

(b) $i^{01}+i^{12}=i^{02}$.

(c) $i^{01}=i_{\gamma^{12} ; \alpha_{0}}^{02}$; that is, $i^{01}$ is the least $i^{\prime}$ such that $\gamma^{12} \in I_{\alpha_{2} ; \alpha_{0} i^{\prime}}^{02}$.

(d) if $i=i^{01}=i^{02}$ (which holds iff $i^{12}=0$ iff $\left(\gamma^{12}, \alpha_{2}\right]_{\mathcal{X}_{2}} \cap \mathscr{D}^{\mathcal{X}_{2}}=\emptyset$ ) then

$$
\tau_{\alpha_{2} ; \alpha_{0} i}^{02}=\tau_{\alpha_{2} ; \alpha_{1} 0}^{12} \circ \tau_{\alpha_{1} ; \alpha_{0} i}^{01}
$$

(e) Suppose $i^{01}<i^{02}$ (which holds iff $i^{12}>0$ iff $\left(\gamma^{12}, \alpha_{2}\right] \mathcal{X}_{2} \cap \mathscr{D}^{\mathcal{X}_{2}} \neq \emptyset$ iff $\left.M_{\alpha_{0} i^{02}}^{\mathcal{X}_{0}} \triangleleft M_{\alpha_{0} i^{01}}^{\mathcal{X}_{0}}\right)$. Then $M_{\alpha_{1} i^{12}}^{\mathcal{X}_{1}}=\tau_{\alpha_{1} ; \alpha_{0} i^{01}}^{01}\left(M_{\alpha_{0} i^{02}}^{\mathcal{X}_{0}}\right)$ and

$$
\tau_{\alpha_{2} ; \alpha_{0} i^{02}}^{02}=\tau_{\alpha_{2} ; \alpha_{1} i^{12}}^{12} \circ\left(\tau_{\alpha_{1} ; \alpha_{0} i^{01}}^{01}\left\lceil M_{\alpha_{0} i^{02}}^{\mathcal{X}_{0}}\right) .\right.
$$

Therefore if also $\ln \left(\mathcal{X}_{2}\right)=\alpha_{2}+1$ and $\operatorname{lh}\left(\mathcal{X}_{1}\right)=\alpha_{1}+1$ (so $\alpha_{0}+1=\operatorname{lh}\left(\mathcal{X}_{0}\right)$ and $i^{02}=i^{01}=0=i^{12}$, because $\mathcal{X}_{1}$ is non- $\mathcal{X}_{0}$-pending), then

$$
\pi_{\infty}^{02}=\pi_{\infty}^{12} \circ \pi_{\infty}^{01}
$$

${ }^{40}$ This does not imply that $\alpha_{2} \in C^{02}$, so $\alpha_{0}$ might not be defined, although $f^{01}\left(\alpha_{1}\right)$ is. 
We literally only prove the fine version; the coarse version is easier.

Proof of Lemma 6.2. By induction on $\operatorname{lh}\left(\mathcal{X}_{2}\right)$. Fix $\alpha_{2}+1<\operatorname{lh}\left(\mathcal{X}_{2}\right)$ and suppose that the lemma holds with respect to $\mathcal{X}_{2} \uparrow\left(\alpha_{2}+1\right)$. We consider three cases.

CASE $1 . \alpha_{2}$ is an $\mathcal{X}_{1}$-copying stage of $\mathcal{X}_{2}$, and $\alpha_{1}$ is an $\mathcal{X}_{0}$-copying stage of $\mathcal{X}_{1}$ (that is, $\alpha_{2} \in\left(C^{-}\right)^{12}$ and $t^{12}\left(\alpha_{2}\right)=0$ and $\alpha_{1} \in\left(C^{-}\right)^{01}$ and $t^{01}\left(\alpha_{1}\right)=0$ ).

We first verify that $\alpha_{2} \in C^{02}$, and establish some other facts. Let $\alpha_{0}^{\prime}=$ $f^{01}\left(\alpha_{1}\right)$. (We don't yet know $\alpha_{2} \in C^{02}$, so we don't yet write $\alpha_{0}$.) We have $\delta_{\alpha_{2} ; \alpha_{1}}^{12}=\alpha_{2}$ and $\delta_{\alpha_{1} ; \alpha_{0}^{\prime}}^{01}=\alpha_{1}$. Let $\bar{\gamma}=\gamma_{\alpha_{1} ; \alpha_{0}^{\prime}}^{01}$ and $\gamma=\gamma_{\alpha_{2} ; \bar{\gamma}}^{12}$ and $\hat{\gamma}=\gamma_{\alpha_{2} ; \alpha_{1}}^{12}$. Since $\bar{\gamma} \leq^{\mathcal{X}_{1}} \alpha_{1}$, we have $\gamma \leq^{\mathcal{X}_{2}} \hat{\gamma}$. And $\hat{\gamma} \in C^{02}$ by property 3(b) (applied with $\beta=\alpha_{0}^{\prime}$ and $\xi=\alpha_{1}$ ), so $[0, \hat{\gamma}]_{\mathcal{X}_{2}} \subseteq C^{02}$, so $\gamma \in C^{02}$. By $4.29(9), \bar{\gamma} \in C^{01}$ and $\alpha_{0}^{\prime}=f^{01}(\bar{\gamma})$ and $\bar{\gamma}=\gamma_{\bar{\gamma} ; \alpha_{0}^{\prime}}^{01}$, and likewise, $\gamma \in C^{12}$ and $\bar{\gamma}=f^{12}(\gamma)$ and $\gamma=\gamma_{\gamma ; \bar{\gamma}}^{12}$. Since $\gamma \in C^{02}$, therefore by induction with property 4(a) (applied with $\gamma$ replacing $\alpha_{2}$ ), we have

$$
\begin{gathered}
\alpha_{0}^{\prime}=f^{02}(\gamma)=f^{01}\left(f^{12}(\gamma)\right) \text { and } \gamma=\gamma_{\gamma ; \alpha_{0}^{\prime}}^{02}=\gamma_{\gamma ; \bar{\gamma}}^{12}, \\
\pi_{\alpha_{1} ; \alpha_{0}^{\prime}}^{01}=\pi_{\bar{\gamma} ; \alpha_{0}^{\prime}}^{01}: M_{\alpha_{0}^{\prime}}^{\mathcal{X}_{0}} \rightarrow M_{\bar{\gamma}}^{\mathcal{X}_{1}}, \\
\pi_{\alpha_{2} ; \bar{\gamma}}^{12}=\pi_{\gamma ; \bar{\gamma}}^{12}: M_{\bar{\gamma}}^{\mathcal{X}_{1}} \rightarrow M_{\gamma}^{\mathcal{X}_{2}}, \\
\pi_{\gamma ; \alpha_{0}^{\prime}}^{02}: M_{\alpha_{0}^{\prime}}^{\mathcal{X}_{0}} \rightarrow M_{\gamma}^{\mathcal{X}_{2}}, \\
\pi_{\gamma ; \alpha_{0}^{\prime}}^{02}=\pi_{\alpha_{2} ; \bar{\gamma}}^{12} \circ \pi_{\alpha_{1} ; \alpha_{0}^{\prime}}^{01} .
\end{gathered}
$$

We have $t^{02}(\xi)=1$ for all $\xi+1 \in\left(\gamma, \alpha_{2}\right]_{\mathcal{X}_{2}}$. For otherwise, by induction (property 2),

$$
\xi \in\left(C^{-}\right)^{12} \text { and } t^{12}(\xi)=0 \text { and } \zeta=f^{12}(\xi) \in\left(C^{-}\right)^{01} \text { and } t^{01}(\zeta)=0 .
$$

So $\xi+1=\gamma_{\alpha_{2} ; \zeta+1}^{12}$ and $\zeta+1 \in\left(\bar{\gamma}, \alpha_{1}\right]_{\mathcal{X}_{1}}$. But $\left[\bar{\gamma}, \alpha_{1}\right]_{\mathcal{X}_{1}}=I_{\alpha_{1} ; \alpha_{0}^{\prime}}^{01}$, so then $t^{01}(\zeta)=1$, contradiction. Let $Q_{0}=\operatorname{ex}_{\alpha_{0}^{\prime}}^{\mathcal{X}_{0}^{\prime}}$ and $\bar{Q}=\pi_{\alpha_{1} ; \alpha_{0}^{\prime}}^{01}\left(Q_{0}\right)$. So to verify $\alpha_{2} \in C^{02}$ we just need to see that $\left(\gamma, \alpha_{2}\right]_{\mathcal{X}_{2}}$ does not drop strictly below the iteration image of

$$
Q={ }_{\operatorname{def}} \pi_{\gamma ; \alpha_{0}^{\prime}}^{02}\left(Q_{0}\right)=\pi_{\alpha_{2} ; \bar{\gamma}}^{12} \circ \pi_{\alpha_{1} ; \alpha_{0}^{\prime}}^{01}\left(Q_{0}\right)=\pi_{\alpha_{2} ; \bar{\gamma}}^{12}(\bar{Q}) .
$$

Note that $j_{\widehat{\gamma} \alpha_{2}}^{\mathcal{X}_{2}}$ is defined, as $\left[\widehat{\gamma}, \alpha_{2}\right]_{\mathcal{X}_{2}}=I_{\alpha_{2} ; \alpha_{1}}^{12}$ (we only defined such embeddings for such intervals), and $\operatorname{dom}\left(j_{\widehat{\gamma} \alpha_{2}}^{\mathcal{X}_{2}}\right)$ is in the dropdown sequence of $\left(M_{\widehat{\gamma}}^{\mathcal{X}_{2}}, \pi_{\alpha_{2} ; \alpha_{1}}^{12}\left(\operatorname{ex}_{\alpha_{1}}^{\mathcal{X}_{1}}\right)\right)$. Likewise, $j_{\bar{\gamma} \alpha_{1}}^{\mathcal{X}_{1}}$ is defined, with $A=\operatorname{dom}\left(j_{\bar{\gamma} \alpha_{1}}^{\mathcal{X}_{1}}\right)$ in the dropdown sequence of $\left(M_{\bar{\gamma}}^{\mathcal{X}_{1}}, \pi_{\alpha_{1} ; \alpha_{0}^{\prime}}^{01}\left(\operatorname{ex}_{\alpha_{0}^{\prime}}^{\mathcal{X}_{0}}\right)\right)$; in fact for each $\beta \in\left[\bar{\gamma}, \alpha_{1}\right]_{\mathcal{X}_{1}}, \operatorname{dom}\left(j_{\bar{\gamma} \beta}^{\mathcal{X}_{1}}\right)$ is in this dropdown sequence. Let $A^{\prime}=\pi_{\alpha_{2} ; \bar{\gamma}}^{12}(A)$ (where $A^{\prime}=M_{\gamma}^{\mathcal{X}_{2}}$ if $A=M_{\bar{\gamma}}^{\mathcal{X}_{1}}$ ) and

$$
k_{\gamma \widehat{\gamma}}^{\mathcal{X}_{2}}: A^{\prime} \rightarrow M_{\widehat{\gamma}}^{\mathcal{X}_{2}}
$$


be the composition of iteration maps along $(\gamma, \widehat{\gamma}] \mathcal{X}_{2}$. This makes sense and we get

$$
\pi_{\alpha_{2} ; \alpha_{1}}^{12} \circ j_{\bar{\gamma} \alpha_{1}}^{\mathcal{X}_{1}}=k_{\gamma \widehat{\gamma}}^{\mathcal{X}_{2}} \circ \pi_{\alpha_{2} ; \bar{\gamma}}^{12}
$$

by the commutativity of tree embedding maps with iteration maps, and preservation of dropping segments under tree embedding maps. Since $t^{01}\left(\alpha_{1}\right)=0$ and $\alpha_{1}=\delta_{\alpha_{1} ; \alpha_{0}^{\prime}}^{01}$,

$$
\operatorname{ex}_{\alpha_{1}}^{\mathcal{X}_{1}}=Q_{1}={ }_{\operatorname{def}} j_{\bar{\gamma} \alpha_{1}}^{\mathcal{X}_{1}}(\bar{Q}) .
$$

Since $t^{12}\left(\alpha_{2}\right)=\alpha_{1}$ and $\alpha_{2}=\delta_{\alpha_{2} ; \alpha_{1}}^{12}$, letting $\widehat{Q}=\pi_{\alpha_{2} ; \alpha_{1}}^{12}\left(Q_{1}\right)$,

$$
\operatorname{ex}_{\alpha_{2}}^{\mathcal{X}_{2}}=Q_{2}={ }_{\operatorname{def}} j_{\widehat{\gamma} \alpha_{2}}^{\mathcal{X}_{2}}(\widehat{Q})
$$

and in particular, $\left(\widehat{\gamma}, \alpha_{2}\right]_{\mathcal{X}_{2}}$ does not drop below the iteration image of $\widehat{Q}$. But by line (3),

$$
\widehat{Q}=k_{\gamma \widehat{\gamma}}^{\mathcal{X}_{2}}\left(\pi_{\alpha_{2} ; \bar{\gamma}}^{12}(\bar{Q})\right)=k_{\gamma \widehat{\gamma}}^{\mathcal{X}_{2}}(Q) .
$$

So $\left[\gamma, \alpha_{2}\right) \mathcal{X}_{2}$ does not drop below the image of $Q$, as desired.

So $\alpha_{2} \in C^{02}$, so by induction, properties 1,4 and 5 hold for $\alpha_{2}$, and in particular, $\alpha_{0}=f^{02}\left(\alpha_{2}\right)=\alpha_{0}^{\prime}$. Since $\alpha_{1} \in\left(C^{-}\right)^{01}$, we have $\alpha_{0}+1<\operatorname{lh}\left(\mathcal{X}_{0}\right)$, so $\alpha_{2} \in\left(C^{-}\right)^{02}$. And since $t^{01}\left(\alpha_{1}\right)=0$ and $t^{12}\left(\alpha_{2}\right)=0$, property 5 (note in particular its parts (d) and (e)) implies $E_{\alpha_{2}}^{\mathcal{X}_{2}}=E_{\alpha_{2}}^{\mathcal{X}_{0} \rightsquigarrow \mathcal{X}_{2}}$, so $t^{02}\left(\alpha_{2}\right)=0$, completing the proof of property 2 . The same property also gives

$$
\omega_{\alpha_{2} ; \alpha_{0}}^{02}=\omega_{\alpha_{2} ; \alpha_{1}}^{12} \circ \omega_{\alpha_{1} ; \alpha_{0}}^{01}
$$

(including that these maps have the same domain and codomain). And note that $\alpha_{2}+1 \in C^{02} \cap C^{12}$ and $\alpha_{1}+1 \in C^{01}$, and properties 1 and 3 at $\alpha_{2}+1$ follow immediately.

We now verify property 4 for $\alpha_{2}+1$. Now $\gamma_{\alpha_{2}+1 ; \alpha_{0}+1}^{02}=\alpha_{2}+1=\gamma_{\alpha_{2}+1 ; \alpha_{1}+1}^{12}$ and $\gamma_{\alpha_{1}+1 ; \alpha_{0}+1}^{01}=\alpha_{1}+1$, by definition of the one-step copy extension. So because of the agreement between $\Pi_{\alpha_{2}+1}^{02}$ and $\Pi_{\alpha_{2}}^{02}$, etc, and by induction, it easily suffices to see that

$$
\pi_{\alpha_{2}+1 ; \alpha_{0}+1}^{02}=\pi_{\alpha_{2}+1 ; \alpha_{1}+1}^{12} \circ \pi_{\alpha_{1}+1 ; \alpha_{0}+1}^{01} .
$$

Let $\xi_{0}=\operatorname{pred}^{\mathcal{X}_{0}}\left(\alpha_{0}+1\right)$ and $\kappa_{0}=\operatorname{cr}\left(E_{\alpha_{0}}^{\mathcal{X}_{0}}\right)$. So $M_{\alpha_{0}+1}^{* \mathcal{X}_{0}}=M_{\xi_{0} \kappa_{0}}^{\mathcal{X}_{0}}$. As $t^{01}\left(\alpha_{1}\right)=0$ (recall the definitions of $\gamma_{\Pi \xi \kappa}, P_{\Pi \xi \kappa}, \pi_{\Pi \xi \kappa}$ from 4.13),

$$
\begin{gathered}
\operatorname{pred}^{\mathcal{X}_{1}}\left(\alpha_{1}+1\right)=\xi_{1}={ }_{\text {def }} \gamma_{\alpha_{1} ; \xi_{0} \kappa_{0}}^{01} \in I_{\alpha_{1} ; \xi_{0}}^{01}, \\
M_{\alpha_{1}+1}^{* \mathcal{X}_{1}}=P_{\alpha_{1} ; \xi_{0} \kappa_{0}}^{01} .
\end{gathered}
$$

Let $\pi^{01}=\pi_{\alpha_{1} ; \xi_{0} \kappa_{0}}^{01}$ and $\kappa_{1}=\pi^{01}\left(\kappa_{0}\right)=\operatorname{cr}\left(E_{\alpha_{1}}^{\mathcal{X}_{1}}\right)$. We have

$$
\begin{gathered}
\operatorname{pred}^{\mathcal{X}_{2}}\left(\alpha_{2}+1\right)=\xi_{2}={ }_{\operatorname{def}} \gamma_{\alpha_{2} ; \xi_{0} \kappa_{0}}^{02}=\gamma_{\alpha_{2} ; \xi_{1} \kappa_{1}}^{12} \in I_{\alpha_{2} ; \xi_{0}}^{02} \cap I_{\alpha_{2} ; \xi_{1}}^{12}, \\
M_{\alpha_{2}+1}^{* \mathcal{X}_{2}}=P_{\alpha_{2} ; \xi_{0} \kappa_{0}}^{02}=P_{\alpha_{2} ; \xi_{1} \kappa_{1}}^{12},
\end{gathered}
$$


with the equalities holding because $t^{02}\left(\alpha_{2}\right)=t^{12}\left(\alpha_{2}\right)=t^{01}\left(\alpha_{1}\right)=0$ and inflations can be freely extended. Let $\pi^{12}=\pi_{\alpha_{2} ; \xi_{1} \kappa_{1}}^{12}$ and $\pi^{02}=\pi_{\alpha_{2} ; \xi_{0} \kappa_{0}}^{02}$, so $\pi^{02}\left(\kappa_{0}\right)=\operatorname{cr}\left(E_{\alpha_{2}}^{\mathcal{X}_{2}}\right)=\pi^{12}\left(\kappa_{1}\right)$. Using part 5 (with $\xi_{2}$ in place of $\alpha_{2}$; note that $\left.\xi_{2} \in C^{02}\right)$, it is now easy to verify that $\pi^{02}=\pi^{12} \circ \pi^{01}$. But $\pi_{\alpha_{2}+1 ; \alpha_{0}+1}^{02}$, etc, are defined as in the proof of the Shift Lemma from $\pi^{02}$ and $\omega_{\alpha_{2} ; \alpha_{0}}^{02}$, etc. So line (5) follows from this commutativity and line (4).

Finally note that part 5 for $\alpha_{2}+1$ follows immediately by induction and from part 4 , because for the new ordinal $\alpha_{2}+1$, with notation as in part 5 , we have $\gamma^{02}=\alpha_{2}+1, i^{02}=0$, etc, so $\tau_{\alpha_{2}+1 ; \alpha_{0}+1,0}^{02}=\pi_{\alpha_{2}+1 ; \alpha_{0}+1}^{02}$, etc.

This completes the induction step in this case.

CASE 2. $\alpha_{2}$ is $\mathcal{X}_{1}$-inflationary (that is, $t^{12}\left(\alpha_{2}\right)=1$ ).

Then $t^{02}\left(\alpha_{2}\right)=1$, so part 2 holds. For if $\alpha_{2} \in\left(C^{-}\right)^{02}$ then by induction, $\alpha_{2} \in C^{12}$ and $\alpha_{1} \in C^{01}$ and $f^{01}\left(\alpha_{1}\right)=\alpha_{0}$, hence $\alpha_{1} \in\left(C^{-}\right)^{01}$, but then since $\mathcal{X}_{1}$ is non- $\mathcal{X}_{0}$-pending, $\alpha_{1}+1<\operatorname{lh}\left(\mathcal{X}_{1}\right)$, so $\alpha_{2} \in\left(C^{-}\right)^{12}$ and $\operatorname{ind}\left(E_{\alpha_{1}}^{\mathcal{X}_{1}}\right) \leq$ $\operatorname{ind}\left(E_{\alpha_{1}}^{\mathcal{X}_{0} \rightsquigarrow \mathcal{X}_{1}}\right)$, so $\left(\operatorname{as} t^{12}\left(\alpha_{2}\right)=1\right) \operatorname{ind}\left(E_{\alpha_{2}}^{\mathcal{X}_{2}}\right)<\operatorname{ind}\left(E_{\alpha_{2}}^{\mathcal{X}_{1} \rightsquigarrow \mathcal{X}_{2}}\right) \leq \operatorname{ind}\left(E_{\alpha_{2}}^{\mathcal{X}_{2} \rightsquigarrow \mathcal{X}_{2}}\right)$ by commutativity. Let $\xi_{2}=\operatorname{pred}^{\mathcal{X}_{2}}\left(\alpha_{2}+1\right)$.

Part 1: Suppose $\alpha_{2}+1 \in C^{02}$. Then $\xi_{2} \in C^{02}$; let $\xi_{0}=f^{02}\left(\xi_{2}\right)$ and $\xi_{1}=f^{12}\left(\xi_{2}\right)$, so also $\xi_{1} \in C^{01}$ and $\xi_{0}=f^{01}\left(\xi_{1}\right)$. And $E_{\alpha_{2}}^{\mathcal{X}_{2}}$ is total over $Q_{\xi_{2} ; \xi_{0}}^{02}$. But if $\xi_{1}+1<\operatorname{lh}\left(\mathcal{X}_{1}\right)$ then $\operatorname{ex}_{\xi_{1}}^{\mathcal{X}_{1}} \unlhd Q_{\xi_{1} ; \xi_{0}}^{01}$ and if $\xi_{1}+1=\ln \left(\mathcal{X}_{1}\right)$ then (because $\mathcal{X}_{1}$ is non- $\mathcal{X}_{0}$-pending) $\xi_{0}+1=\ln \left(\mathcal{X}_{0}\right)$ and $M_{\xi_{1}}^{\mathcal{X}_{1}}=Q_{\xi_{1} ; \xi_{0}}^{01}$. So $Q_{\xi_{2} ; \xi_{1}}^{12} \unlhd Q_{\xi_{2} ; \xi_{0}}^{02}$. So $E_{\alpha_{2}}^{\mathcal{X}_{2}}$ is total over $Q_{\xi_{2} ; \xi_{1}}^{12}$. So $\alpha_{2}+1 \in C^{12}$ and $f^{12}\left(\alpha_{2}+1\right)=f^{12}\left(\xi_{2}\right)=\xi_{1} \in C^{01}$. Likewise $f^{02}\left(\alpha_{2}+1\right)=\xi_{0}$, giving part 1 .

Parts 3 and 4 are easy by induction.

Part 5: Suppose $\alpha_{2}+1 \in C^{02}$ and continue with the notation above. Now $\Pi_{\alpha_{2}+1}^{i 2}$ is the $E_{\alpha_{2}}^{\mathcal{X}_{2}}$-inflation of $\Pi_{\xi_{2}}^{i 2}$ for $i=0,1$. But then property 5 at $\alpha_{2}+1$ follows easily from the same property at $\xi_{2}$; we get the instance of Figure 5 at stage $\alpha_{2}+1$, from that at stage $\xi_{2}$, by simply adding one further step of iteration above $M_{\alpha_{2}+1}^{* \mathcal{X}_{2}} \unlhd M_{\xi_{2}}^{\mathcal{X}_{2}}$ (at the top of the diagram). (This possibly inflicts a drop in model, but because $\alpha_{2}+1 \in C^{02}$, hence also $\alpha_{2}+1 \in C^{12}$, we do not drop too far; the integer $i^{01}$ is not modified, and the integers $i^{02}$ and $i^{12}$ are modified by the same amount.)

CASE 3. $\alpha_{2}$ is $\mathcal{X}_{1}$-copying but $\alpha_{1}$ is $\mathcal{X}_{0}$-inflationary (that is, $\alpha_{2} \in\left(C^{-}\right)^{12}$ and $t^{12}\left(\alpha_{2}\right)=0$ but $\left.t^{01}\left(\alpha_{1}\right)=1\right)$.

We have $\alpha_{2}+1 \in C^{12}$ and $f^{12}\left(\alpha_{2}+1\right)=\alpha_{1}+1$ and $\gamma_{\alpha_{2}+1 ; \alpha_{1}+1}^{12}=\alpha_{2}+1$. And $t^{02}\left(\alpha_{2}\right)=1$ for reasons much as before, giving part 2. Let $\xi_{i}=\operatorname{pred}^{\mathcal{X}_{i}}\left(\alpha_{i}+1\right)$ for $i=1,2$. Then $\xi_{2} \in C^{12}$ and $f^{12}\left(\xi_{2}\right)=\xi_{1}$. By commutativity at stage $\xi_{2}$, we easily have $\alpha_{2}+1 \in C^{02}$ iff $\alpha_{1}+1 \in C^{01}$; and if $\alpha_{2}+1 \in C^{02}$ then, letting $\xi_{0}=f^{02}\left(\xi_{2}\right)=f^{01}\left(\xi_{1}\right)$, we have $f^{02}\left(\alpha_{2}+1\right)=\xi_{0}=f^{01}\left(\alpha_{1}+1\right)$, since $t^{02}\left(\alpha_{2}\right)=t^{01}\left(\alpha_{1}\right)=1$. So part 1 holds.

Parts 3 and 4 are again easy. (In part 3(b), for $\alpha_{2}+1$ and $\beta=\xi_{0}$ and $\xi=\alpha_{1}+1 \in I_{\alpha_{1}+1 ; \xi_{0}}^{01}$, we have $\gamma_{\alpha_{2}+1 ; \alpha_{1}+1}^{12}=\alpha_{2}+1 \in C^{02}$, as required.) And part 5 is again straightforward by induction; we obtain the diagram at stage $\alpha_{2}+1$ by adding a commuting square to the top of diagram from stage $\xi_{2}$, applying the extenders $E_{\alpha_{1}}^{\mathcal{X}_{1}}$ and $E_{\alpha_{2}}^{\mathcal{X}_{2}}$ to $M_{\alpha_{1}+1}^{* \mathcal{X}_{1}}$ and $M_{\alpha_{2}+2}^{* \mathcal{X}_{2}}$ respectively; in the 
new diagram the upper triangle collapses.

This completes the successor case. The limit case is a simplification thereof. Suppose that the lemma holds with regard to $\mathcal{X}_{2}\lceil\eta$, where $\eta$ is a limit, and we want to prove it for $\mathcal{X}_{2}\lceil\eta+1$. There are again three cases, analogous to those in the successor case. For an inflation $\mathcal{T} \rightsquigarrow \mathcal{X}$, with associated objects $C, f$, and a limit $\eta<\operatorname{lh}(\mathcal{X})$, say that $\eta$ is a $(\mathcal{T}, \mathcal{X})$-limit iff $\eta \in C$ and $f(\alpha)<f(\eta)$ for all $\alpha<^{\mathcal{X}} \eta$. Then either:

1. $\eta$ is an $\left(\mathcal{X}_{0}, \mathcal{X}_{2}\right)$-limit. Then easily by induction, $\eta$ is also an $\left(\mathcal{X}_{1}, \mathcal{X}_{2}\right)$-limit and $f^{12}(\eta)$ is an $\left(\mathcal{X}_{0}, \mathcal{X}_{1}\right)$-limit. This is analogous to Case 1 (an $\mathcal{X}_{0}$-copying (and $\mathcal{X}_{1}$-copying) stage of $\mathcal{X}_{2}$ ).

2. $\eta$ is not an $\left(\mathcal{X}_{1}, \mathcal{X}_{2}\right)$-limit. So $\eta$ is also not an $\left(\mathcal{X}_{0}, \mathcal{X}_{2}\right)$-limit. (Analogous to Case 2 , an $\mathcal{X}_{1}$-inflationary stage of $\mathcal{X}_{2}$.)

3. $\eta$ is an $\left(\mathcal{X}_{1}, \mathcal{X}_{2}\right)$-limit, but not an $\left(\mathcal{X}_{0}, \mathcal{X}_{2}\right)$-limit. Then $f^{12}(\eta)$ is not an $\left(\mathcal{X}_{0}, \mathcal{X}_{1}\right)$-limit. (Analogous to Case 3 , an $\mathcal{X}_{1}$-copying, $\mathcal{X}_{0}$-inflationary stage of $\mathcal{X}_{2}$.)

In each case, the properties follow easily from the commutativity given by induction. We leave the details to the reader.

An easy consequence is:

6.3 Corollary. Let $\mathcal{X}_{0}, \mathcal{X}_{1}, \mathcal{X}_{2}$ be as in 6.2. Suppose that $\mathcal{X}_{2}$ is $\mathcal{X}_{1}$-terminal and $\mathcal{X}_{1}$ is $\mathcal{X}_{0}$-terminal. Then $\mathcal{X}_{2}$ is $\mathcal{X}_{0}$-terminal. Moreover, $\mathcal{X}_{2}$ is $\mathcal{X}_{0}$-terminally(model-)dropping iff either $\mathcal{X}_{1}$ is $\mathcal{X}_{0}$-terminally-(model-)dropping or $\mathcal{X}_{2}$ is $\mathcal{X}_{1}$ terminally-(model-)dropping.

The author was initially focused on inflation (as opposed to tree embeddings more generally), and did not notice that the preceding lemma has the following natural variant, until it was pointed out by Jensen. It follows from part of the proof of 6.2 :

6.4 Lemma (Composition of tree embeddings). Let $\mathcal{X}_{i}$ be u-m-maximal trees for $i=0,1,2$. Let $\Pi_{i, i+1}: \mathcal{X}_{i} \hookrightarrow \mathcal{X}_{i+1}$ be a tree embedding, for $i=0,1$. Then $\Pi_{02}: \mathcal{X}_{0} \hookrightarrow \mathcal{X}_{2}$ is a tree embedding, where writing $\gamma_{\alpha}^{i j}=\gamma_{\Pi_{i j} \alpha}$, etc, we have

$$
\gamma_{\alpha}^{02}=\gamma_{\gamma_{\alpha}^{01}}^{12} \text { and } \delta_{\alpha}^{02}=\delta_{\delta_{\alpha}^{01}}^{12} \text {. }
$$

for each $\alpha<\operatorname{lh}\left(\mathcal{X}_{0}\right)$. Moreover, for each $\alpha<\operatorname{lh}\left(\mathcal{X}_{0}\right)$ we have

$$
\pi_{\alpha}^{02}=\pi_{\gamma_{\alpha}^{01}}^{12} \circ \pi_{\alpha}^{01} \text { and } \omega_{\alpha}^{02}=\omega_{\delta_{\alpha}^{01}}^{12} \circ \omega_{\alpha}^{01} .
$$

\section{Generic absoluteness of iterability}

We establish in this section some general theorems on the absoluteness of iterability under forcing. Let $M$ be an $m$-sound premouse. Let $\Omega>\omega$ be regular and let $V[G]$ be a generic extension of $V$ via an $\Omega$-cc forcing. In the main result 
(Theorem 7.3), assuming that $\Sigma$ is an $(m, \Omega+1)$-strategy for $M$ with strong hull condensation, we extend $\Sigma$ to $\Sigma^{\prime}$, such that in $V[G], \Sigma^{\prime}$ is an $(m, \Omega+1)$ strategy with strong hull condensation. (We do not know whether the analogous statement can be proved for inflation condensation.) This holds for both wcpms and seg-pms, of arbitrary cardinality. If $M$ is a countable premouse and $e$ an $\omega$-enumeration of $M$ and $\Sigma$ has weak DJ with respect to $e$, then so does $\Sigma^{\prime}$. We also use the result to obtain a universally Baire representation for $\Sigma \uparrow \mathrm{HC}$, assuming that $M$ is also countable (see $§ 7.2$ ). In the other direction (Corollary 7.6), assume that $M$ is countable in $V$ and $\Sigma^{\prime}$ has weak DJ in $V[G]$ with respect to some enumeration $e \in V$; then $\Sigma=\Sigma^{\prime}\lceil V \in V$. The proof involves standard kinds of arguments and is probably part of the folklore, but we give it. Thus, if $M$ is a countable premouse and $e \in V$ an $\omega$-enumeration of $M$, then the existence of an $(m, \Omega+1)$-strategy for $M$ with weak DJ with respect to $e$ is absolute between $V$ and $V[G]$. Combined with the results later in the paper, we will also get that if $V \models$ ZFC and $M$ is countable, then the existence of an $(m, \Omega+1)$-strategy for $M$ with strong hull condensation is absolute between $V$ and $V[G]$; this is because under DC, given such a strategy and an enumeration $e$, we can construct a strategy with weak DJ with respect to $e$.

\subsection{Extending strategies to generic extensions}

The background theory here, as elsewhere, is ZF. Thus, we specify exactly what we mean by the $\Omega$-chain condition:

7.1 Definition. Let $\mathbb{P}$ be a poset and $\lambda \in$ OR. A $\lambda$-pre-antichain of $\mathbb{P}$ is a partition $\left\langle A_{\alpha}\right\rangle_{\alpha<\lambda}$ of some set $A \subseteq \mathbb{P}$ such that each $A_{\alpha} \neq \emptyset$, and $p \perp q$ whenever $p \in A_{\alpha}$ and $q \in A_{\beta}$ for some $\alpha<\beta<\lambda$. We say that $\mathbb{P}$ has the $\lambda$-cc iff there is no $\lambda$-pre-antichain of $\mathbb{P}$.

7.2 Remark. Clearly the above definition agrees with the usual definition of $\lambda$-cc under ZFC. The usual ZFC argument easily adapts to show under ZF that if $\lambda$ is regular then forcing with a $\lambda$-cc forcing preserves the regularity of $\lambda$.

7.3 Theorem. Let $\Omega>\omega$ be regular. Let $\mathbb{P}$ be an $\Omega$-cc forcing and $G$ be $V$ generic for $\mathbb{P}$. Let $M$ be an $\ell$-sound premouse, or let $M$ be a wcpm and $\ell=0$. Let $\Gamma$ be an $(\ell, \Omega+1)$-strategy ${ }^{41}$ for $M$ with strong hull condensation. Then:

1. In $V[G]$ there is a unique $(\ell, \Omega+1)$-strategy $\Gamma^{\prime}$ such that $\Gamma \subseteq \Gamma^{\prime}$ and $\Gamma^{\prime}$ has inflation condensation.

2. In $V[G], \Gamma^{\prime}$ has strong hull condensation.

3. Suppose $M \in \mathrm{HC}$ is a premouse (not a wcpm) and let e be an enumeration of $M$ in ordertype $\omega$. Then:

- $\Gamma$ has Dodd-Jensen iff $\Gamma^{\prime}$ has Dodd-Jensen in $V[G]$.

\footnotetext{
${ }^{41}$ Recall that if $M$ is a wcpm, this just means an $(\Omega+1)$-strategy.
} 
- $\Gamma$ has weak Dodd-Jensen with respect to $e$ iff $\Gamma^{\prime}$ has weak DoddJensen with respect to $e$ in $V[G]$.

Further, let $\Sigma$ be the u-strategy corresponding to $\Gamma$ and $m=m^{\Sigma} .{ }^{42}$ Then:

4. In $V[G]$ there is a unique $(\mathrm{u}-m, \Omega+1)$-strategy $\Sigma^{\prime}$ such that $\Sigma \subseteq \Sigma^{\prime}$ and $\Sigma^{\prime}$ has inflation condensation.

5. In $V[G], \Sigma^{\prime}$ has strong hull condensation.

6. If $M$ is $M S$-indexed then in $V[G], \Sigma^{\prime}$ is the u-strategy corresponding to $\Gamma^{\prime}$.

7. For every tree $\mathcal{T} \in V[G]$ via $\Sigma^{\prime}$, there is a $\mathcal{T}$-terminally-non-dropping inflation $\mathcal{X}$ of $\mathcal{T}$ such that $\mathcal{X} \in V$ and $\mathcal{X}$ is via $\Sigma$. Moreover, if $\ln (\mathcal{T})<\Omega$ then we can take $\ln (\mathcal{X})<\Omega$.

Proof. We just prove the fine-structural variants; the version for wcpms is a slight simplification. (The key point here is that we do not need to form any standard comparison of premice in the argument, although we do use "comparison" of iteration trees, that is, minimal simultaneous inflation.) We will first prove parts 4,5 and 7; this automatically yields parts 1,2 and 6 , by the correspondence of convenient and inconvenient strategies.

Work in $V[G]$. Let $\Sigma^{\prime}$ be the set of all pairs $(\mathcal{T}, b)$ such that $\mathcal{T}$ is a u- $m$ maximal tree on $M$ of length $\leq \Omega$ and $b$ is $\mathcal{T}$-cofinal and there is a limit length tree $\mathcal{X} \in V$ and $\mathcal{X}$-cofinal branch $c \in V$ with $(\mathcal{X}, c)$ via $\Sigma$, and there is a tree embedding $\Pi:(\mathcal{T}, b) \hookrightarrow(\mathcal{X}, c)$; equivalently by 4.28 , there is an almost tree embedding $\Pi:(\mathcal{T}, b) \hookrightarrow$ alm $(\mathcal{X}, c)$. We will verify that $\Sigma^{\prime}$ is a $(\mathrm{u}-m, \Omega+1)$ strategy for $M$, with strong hull condensation. Actually, for each such $(\mathcal{T}, b)$, we will find a witnessing $(\mathcal{X}, c) \in V$ which is a terminally-non-dropping inflation of $(\mathcal{T}, b)$.

We start by showing that $\Sigma^{\prime}$ is a function.

Claim 6. Let $\mathcal{X}, \mathcal{X}^{\prime} \in V$ be via $\Sigma$. Work in $V[G]$. Let $\Pi:(\mathcal{T}, b) \hookrightarrow \mathcal{X}$ and $\Pi^{\prime}:\left(\mathcal{T}, b^{\prime}\right) \hookrightarrow \mathcal{X}^{\prime}$ be tree embeddings. Then $b=b^{\prime}$.

Proof. Suppose not and fix $\mathcal{X}, \mathcal{X}^{\prime}$. Let $S$ be the tree of attempts to build (a code for) a tuple $\left(\mathcal{T}, b, b^{\prime}, \Pi, \Pi^{\prime}\right)$ such that $\mathcal{T}$ is a countable limit length (potential, that is, satisfies the relevant first order requirements, but without demanding that $\ln (\mathcal{T})$ be wellfounded or that $\mathcal{T}$ have wellfounded models) iteration tree on $M$ and $b, b^{\prime}$ are distinct $\mathcal{T}$-cofinal branches, $\Pi:(\mathcal{T}, b) \hookrightarrow$ alm $\mathcal{X}$ and $\Pi^{\prime}$ : $\left(\mathcal{T}, b^{\prime}\right) \hookrightarrow_{\text {alm }} \mathcal{X}^{\prime}$ (and hence, $(\mathcal{T}, b)$ and $\left(\mathcal{T}, b^{\prime}\right)$ are in fact true iteration trees). Here we can and do take $S$ as a tree on some $\lambda \in$ OR. We can do this because an element $s$ of $S$ specifies some finite iteration tree $\overline{\mathcal{T}}_{s}$ on $M$, with domain some finite set $D_{s} \subseteq \omega$, with $D_{s^{\prime}} \subseteq D_{s}$ for $s^{\prime} \unlhd s$, specifies how each $\overline{\mathcal{T}}_{s^{\prime}}$ fits as a subtree of $\overline{\mathcal{T}}_{s}$, and specifies $b \cap D$ and $b^{\prime} \cap D$ and $\Pi\left\lceil D\right.$ and $\Pi^{\prime}\lceil D$ (the latter meaning just $\gamma_{\alpha}, \delta_{\alpha}, \gamma_{\alpha}^{\prime}, \delta_{\alpha}^{\prime}$ for $\left.\alpha \in D\right)$. Here $\overline{\mathcal{T}}_{s}$ can be specified by a finite

\footnotetext{
${ }^{42}$ See 4.36. So if $M$ is not MS-indexed then $\Gamma=\Sigma$ and $m=\ell$.
} 
sequence of ordinals because recall that in the coarse (wcpm) case, although $M$ need not model ZFC, we do demand that the extenders used come from $\mathbb{E}^{M}$, which is a wellordered set.

Now because of our contradictory assumption, $S$ is illfounded in $V^{\mathrm{Col}(\omega, \gamma)}$ for sufficiently large $\gamma$, and therefore $S$ is illfounded in $V$. But then (as $S$ is on $\lambda$ ) we get some such $\mathcal{T}, b, b^{\prime}, \Pi, \Pi^{\prime} \in V$, contradicting strong hull condensation.

As mentioned earlier, whenever $b=\Sigma^{\prime}(\mathcal{T})$, we will actually find a $(\mathcal{T}, b)$ terminally-non-dropping inflation $(\mathcal{X}, c)$ of $(\mathcal{T}, b)$, with $(\mathcal{X}, c) \in V$ and via $\Sigma$. We can actually prove the uniqueness of such $b$ using only inflation condensation, and we give this proof next. However, this uniqueness is not enough for the overall proof; we seem to need the stronger uniqueness of the claim above, which relied on strong hull condensation. So we just include the next claim for interest, and in case one might be able to improve on its proof, so as to replace the use of strong hull condensation in the theorem with inflation condensation. 43

Claim 7 . Let $\mathcal{T}, b_{0}, \mathcal{X}_{0}, c_{0}, b_{1}, \mathcal{X}_{1}, c_{1}$ be such that $\left(\mathcal{X}_{i}, c_{i}\right) \in V$, according to $\Sigma$, is a $\left(\mathcal{T}, b_{i}\right)$-terminally-non-dropping inflation of $\left(\mathcal{T}, b_{i}\right)$. Then $b_{0}=b_{1}$, assuming only inflation condensation for $\Sigma$.

Proof. Let $f^{0}=f^{\left(\mathcal{T}, b_{0}\right) \rightsquigarrow\left(\mathcal{X}_{0}, c_{0}\right)}$, etc. By minimizing $\operatorname{lh}\left(\mathcal{X}_{i}\right)$, we may assume $\mathcal{X}_{i}$ is also an inflation of $\mathcal{T}$, as witnessed by $\widetilde{f}^{0}=f^{\mathcal{T} \rightsquigarrow \mathcal{X}_{0}}$, etc (otherwise replace $\left(\mathcal{X}_{0}, c_{0}\right)$ with $\mathcal{X}_{0}\left\lceil\eta+1\right.$ for the least $\eta$ where $\left.f^{0}(\eta)=\operatorname{lh}(\mathcal{T})\right)$. Then $\widetilde{C}^{i}=C^{i} \cap \eta_{i}$ where $\eta_{i}=\operatorname{lh}\left(\mathcal{X}_{i}\right), \tilde{f}^{i}=f^{i} \uparrow \widetilde{C}^{i}, \eta_{i} \in C^{i}$ and $f^{i}\left(\eta_{i}\right)=\operatorname{lh}\left(\mathcal{T}_{i}\right)$.

In $V$, let $\mathcal{X}$, of length $\lambda+1$, be the least initial segment of the minimal simultaneous inflation $(\S 5.1)$ of $\left(\mathcal{X}_{0}, c_{0}\right)$ and $\left(\mathcal{X}_{1}, c_{1}\right)$ where for some $i \in\{0,1\}$,

$$
\lambda \in C^{\left(\mathcal{X}_{i}, c_{i}\right) \rightsquigarrow \mathcal{X}} \text { and } f^{\left(\mathcal{X}_{i}, c_{i}\right) \rightsquigarrow \mathcal{X}}(\lambda)=\operatorname{lh}\left(\mathcal{X}_{i}\right) .
$$

We may assume $i=0$. Then $\mathcal{X}$ is an $\left(\mathcal{X}_{0}, c_{0}\right)$-terminally-non-dropping inflation of $\left(\mathcal{X}_{0}, c_{0}\right)$. Note $\lambda$ is a limit, and by Corollary $6.3, \mathcal{X}$ is a $\left(\mathcal{T}_{0}, b_{0}\right)$-terminallynon-dropping inflation of $\left(\mathcal{T}_{0}, b_{0}\right)$. Let $\hat{C}^{0}$, etc, be the witnesses to the latter. Then by Lemma $6.2, \lambda \in \hat{C}^{0}$ and $\hat{f}^{0}(\lambda)=\operatorname{lh}(\mathcal{T})$, so $b^{\mathcal{X}}$ determines $b_{0}$ via this inflation. By the minimality of $\lambda, \hat{f}^{0}(\alpha)<\ln (\mathcal{T})$ for each $\alpha \in \lambda \cap \hat{C}^{0}$. So note that $\mathcal{X} \uparrow \lambda$ is an inflation of $\mathcal{T}$, as witnessed by $\hat{C}^{0} \cap \lambda, \hat{f}^{0} \uparrow \lambda$, etc. (The branch $b_{0}$ is irrelevant because $\ln (\mathcal{T}) \notin \hat{f}^{0 \text { " } \lambda \text {.) }}$

Now because $\mathcal{X}$ is also an inflation of $\left(\mathcal{X}_{1}, c_{1}\right)$, by $6.2, \mathcal{X}$ is also an inflation of $\left(\mathcal{T}, b_{1}\right)$, as witnessed by $\hat{C}^{1}$, etc, and again by minimality of $\lambda$, we have $\operatorname{lh}(\mathcal{T}) \notin \hat{f}^{1}$ " $\lambda$. So $\hat{C}^{1} \cap \lambda=\hat{C}^{0} \cap \lambda$ and $\hat{f}^{0} \uparrow \lambda=\hat{f}^{1} \uparrow \lambda$ etc. But then $\hat{C}^{0}=\hat{C}^{1}$ and $\hat{f}^{0}=\hat{f}^{1}$ etc, because the extensions are determined by the common restrictions to $\lambda$ and $\mathcal{T}$ and $b^{\mathcal{X}}$. So $\lambda \in \hat{C}^{1}$ and $\hat{f}^{1}(\lambda)=\operatorname{lh}(\mathcal{T})$ and since $\mathcal{X}$ is an inflation of $\left(\mathcal{T}, b_{1}\right), b^{\mathcal{X}}$ determines $b_{1}$. But $b^{\mathcal{X}}$ determines $b_{0}$, so $b_{0}=b_{1}$. This gives the claim.

\footnotetext{
${ }^{43}$ In an earlier draft of this paper, which was available on the author's website for a short period of time, we had actually stated the theorem with inflation condensation instead of strong hull condensation, but there was a gap in that putative proof.
} 
We now verify that $\Sigma^{\prime}$ produces wellfounded models and is total.

Claim 8. Let $\mathcal{T} \in V[G]$ be a putative tree via $\Sigma^{\prime}$. If $\ln (\mathcal{T})$ is a successor then there is $\mathcal{X} \in V$ via $\Sigma$ and such that $\mathcal{X}$ is a $\mathcal{T}$-terminally-non-dropping inflation of $\mathcal{T}$, and if $\operatorname{lh}(\mathcal{T})<\Omega$ then we can take $\operatorname{lh}(\mathcal{X})<\Omega$; so every such $\mathcal{T}$ is a true iteration tree. If $\ln (\mathcal{T})$ is a limit $(\leq \Omega)$ then $\mathcal{T} \in \operatorname{dom}\left(\Sigma^{\prime}\right)$.

Proof. We prove the claim by induction on $\ln (\mathcal{T})$. Suppose we have a tree $\mathcal{T}$ of length $\eta+1<\Omega$, and the claim holds for $\mathcal{T}$. Fix $\mathcal{X}$ witnessing this. Then for trees $\mathcal{T}^{\prime}$ normally extending $\mathcal{T}$ of length $<\eta+\omega$, we may extend $\mathcal{X}$ to a $\mathcal{T}^{\prime}$-terminally-non-dropping inflation $\mathcal{X}^{\prime}$ of $\mathcal{T}^{\prime}$, by simply copying the finite remainder of $\mathcal{T}^{\prime}$ up, and since $\mathcal{X} \in V$ is via $\Sigma$, so is $\mathcal{X}^{\prime}$.

So fix $\mathcal{T}$ of limit length. We will find some $\mathcal{T}$-cofinal $b \in V[G]$ and a $(\mathcal{T}, b)$ terminally-non-dropping inflation $(\mathcal{X}, c)$ of $(\mathcal{T}, b)$, with $(\mathcal{X}, c) \in V$ via $\Sigma$.

For this, working in $V$, we form a Boolean valued minimal simultaneous inflation of various candidates for $\mathcal{T}$. Fix $p_{0} \in \mathbb{P}$ forcing that $\mathcal{T}$ is as above. We will define the (Boolean valued minimal simultaneous) inflation relative to $p_{0}$, producing a tree $(\mathcal{X}, c)$, and show that there is $q \leq p_{0}$ such that $q$ forces that it works for some $\dot{\mathcal{T}}$-cofinal branch $\dot{b}$. This is enough by density.

So, we define a tree $\mathcal{X}$ on $M$, using extenders $E_{\alpha}^{\mathcal{X}}$ with indices $\xi_{\alpha}$, as follows. Let $E_{0}^{\mathcal{X}}$ be the least $E \in \mathbb{E}_{+}^{M}$ such that some $q \leq p_{0}$ forces that $E_{0}^{\mathcal{T}}=E$. This gives $\mathcal{X}\lceil 2$.

Now suppose we have $\mathcal{X}\left\lceil\alpha+1\right.$. If $\alpha$ is a limit and there is some $q \leq p_{0}$ forcing " $\mathcal{X}\lceil\alpha+1$ is a $(\dot{\mathcal{T}}, b)$-terminally-non-dropping inflation of $(\dot{\mathcal{T}}, b)$ for some $\mathcal{T}$-cofinal $b "$, then we stop the construction (with success). Now suppose otherwise. If $\alpha=\Omega$ then we stop (with failure due to long tree). Suppose otherwise. Let $E_{\alpha}^{\mathcal{X}}$ be the least $E \in \mathbb{E}_{+}\left(M_{\alpha}^{\mathcal{X}}\right)$ such that some $q \leq p_{0}$ forces that $\mathcal{X}\left\lceil\alpha+1\right.$ is an inflation of $\dot{\mathcal{T}}$, with $\alpha \in C^{-}$, and $E=E_{\alpha}^{\dot{\mathcal{T}} \rightsquigarrow \mathcal{X} \mid \alpha+1}$, if such an $E$ exists; otherwise we stop (with failure due to dropping).

At limit stages $\eta$, we extend $\mathcal{X}\lceil\eta$ using $\Sigma$.

This completes the definition of $\mathcal{X}$. We next verify that the construction stops with success.

Now $p_{0}$ forces that $\mathcal{X}$ is an inflation of $\dot{\mathcal{T}}$. This follows from the minimality of $\operatorname{ind}\left(E_{\beta}^{\mathcal{X}}\right)$ for each $\beta$ together with Claim 6 . That is, if $\eta<\ln (\mathcal{X})$ is a limit and $p_{0}$ forces that $\mathcal{X}\left\lceil\eta\right.$ is an inflation of $\dot{\mathcal{T}}$, then $p_{0}$ forces that $\mathcal{X} \uparrow(\eta+1)$ is also an inflation of $\dot{\mathcal{T}}$. For otherwise there are $q, \lambda$ such that $q \leq p_{0}$ and $q$ forces " $\lambda<\operatorname{lh}(\dot{\mathcal{T}})$ and there is $b \neq[0, \lambda)_{\dot{\mathcal{T}}}$ and a tree embedding

$$
\Pi:(\dot{\mathcal{T}}\lceil\lambda, b) \hookrightarrow \mathcal{X} \uparrow(\eta+1), "
$$

contradicting Claim $6 .{ }^{44}$

Now suppose the construction stops with failure due to dropping, giving tree $\mathcal{X}=\mathcal{X}\left\lceil\alpha+1\right.$ (so $\alpha<\Omega$ ). Note $p_{0}$ forces " $\alpha \notin C^{-"}$. Now $\alpha$ is a limit,

\footnotetext{
${ }^{44}$ Note that Claim 7 does not suffice here, because we need to rule out the possibility of having a limit $\lambda<\operatorname{lh}(\mathcal{T})$ and some limit $\eta$ such that $\mathcal{X}\lceil\eta$ is an inflation of $\mathcal{T}$, but $\mathcal{X}\lceil(\eta+1)$ is not, because $[0, \eta)_{\mathcal{X}}$ induces some $\mathcal{T}$-maximal branch which is not $\mathcal{T}$-cofinal. Claim 7 does not suffice to rule this out.
} 
because if $\alpha=\beta+1$ then some $q \leq p_{0}$ forces " $E_{\beta}^{\mathcal{X}}$ is copied from $\dot{\mathcal{T}}$ ", so $q$ forces " $\alpha=\gamma_{\alpha ; f(\alpha)}^{\dot{\mathcal{T}} \boldsymbol{\mathcal { X }})}$, so $\alpha \in C^{-}$(as $\dot{\mathcal{T}}$ has limit length)"; contradiction. So let $\beta<^{\mathcal{X}} \alpha$ be such that $(\beta, \alpha)_{\mathcal{X}}$ does not drop. Some $q \leq p_{0}$ forces " $\beta \in C^{-"}$ ". We claim

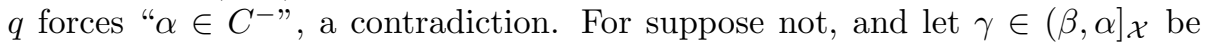
least such that some $r \leq q$ forces " $\gamma \notin C^{-}$", and fix $s \leq r$ such that $s$ decides the values $\lambda=\sup _{\xi<{ }^{\mathcal{X}} \gamma} f(\xi)$ and $\lambda^{\prime}=\operatorname{lh}(\dot{\mathcal{T}})$. Because $(\beta, \alpha)_{\mathcal{X}}$ does not drop, $\gamma$ is a limit ordinal. But then if $\lambda<\lambda^{\prime}$, note that $s$ forces $\gamma \in C$ (recall $p_{0}$ forces that $\mathcal{X}$ is an inflation of $\dot{\mathcal{T}}$, so $s$ forces that $[0, \gamma)_{\mathcal{X}}$ determines $\left.[0, \lambda)_{\dot{\mathcal{T}}}\right)$ and hence $\gamma \in C^{-}$, a contradiction. So $\lambda=\lambda^{\prime}$, but then the construction stops with success at stage $\gamma$, as witnessed by $s$ and the $\dot{\mathcal{T}}$-cofinal branch determined by $[0, \gamma) \mathcal{X}$, a contradiction.

So finally suppose that the process stops with failure due to a long tree, so we get $\mathcal{X}$ of length $\Omega+1$. If $q \leq p_{0}$ and $q$ forces that cofinally many extenders used along $[0, \Omega)_{\mathcal{X}}$ are $\dot{\mathcal{T}}$-copying, then because $\Omega$ is regular in $V[G]$ and $\operatorname{lh}(\dot{\mathcal{T}}) \leq \Omega, q$ forces that $\operatorname{lh}(\dot{\mathcal{T}})=\Omega$ and the process ends successfully at $\alpha=\Omega$, contradiction. But if there is no such $q$, then by $\Omega$-cc-ness, there is some $\alpha<\Omega$ such that $p_{0}$ forces that every extender used along $(\alpha, \Omega)_{\mathcal{X}}$ is $\dot{\mathcal{T}}$-inflationary. But this is impossible, as by construction, for every extender $E$ used in $\mathcal{X}$, there is $q \leq p_{0}$ forcing that $E$ is $\dot{\mathcal{T}}$-copying.

So the construction stops with success, as witnessed by $\alpha \leq \Omega$ and $q \leq p_{0}$ (so $\ln (\mathcal{X})=\alpha+1)$. Finally, to complete the proof of the claim, we show that if $\alpha=\Omega$ then $q$ forces that $\ln (\dot{\mathcal{T}})=\Omega$. But by the minimality of $\alpha$ (that is, there is no $\alpha^{\prime}<\alpha$ such that the construction stopped with success at stage $\left.\alpha^{\prime}\right), q$ forces that " $f(\alpha)<\operatorname{lh}(\dot{\mathcal{T}})$ for all $\alpha<^{\mathcal{X}} \Omega$, and $f(\Omega)=\operatorname{lh}(\dot{\mathcal{T}})$, and $f(\Omega)=\sup _{\alpha<{ }^{\mathcal{X}} \Omega} f(\alpha)$ ", but $\Omega$ is regular in $V[G]$, so $q$ forces $\ln (\dot{\mathcal{T}})=\Omega$.

Claim 9. $\Sigma^{\prime}$ has strong hull condensation.

Proof. Work in $V[G]$. Let $\Pi: \mathcal{T} \hookrightarrow \mathcal{U}$ where $\mathcal{U}$ is via $\Sigma^{\prime}$. We may assume that $\mathcal{U} \in V$ is via $\Sigma$, by 6.4 and Claim 8 . We claim that $\mathcal{T}$ is via $\Sigma^{\prime}$. For let $\eta<\operatorname{lh}(\mathcal{T})$ be a limit and $b=\Sigma^{\prime}(\mathcal{T}\lceil\eta)$. Then using a restriction of $\Pi$ and Claim 6 , we have $b=[0, \eta)_{\mathcal{T}} \cdot{ }^{45}$

Claim 10. In $V[G], \Sigma^{\prime}$ is the unique (u- $\left.m, \Omega+1\right)$-strategy with inflation condensation which extends $\Sigma$.

Proof. In $V[G]$, let $\Sigma^{\prime \prime}$ be such a strategy. Let $\mathcal{T}$ be of limit length, according to both $\Sigma^{\prime}$ and $\Sigma^{\prime \prime}$, and let $b^{\prime}=\Sigma^{\prime}(\mathcal{T})$ and $b^{\prime \prime}=\Sigma^{\prime \prime}(\mathcal{T})$. We need to see that $b^{\prime}=b^{\prime \prime}$. Let $(\mathcal{X}, c) \in V$, according to $\Sigma$, be a $\left(\mathcal{T}, b^{\prime}\right)$-terminal inflation of $\left(\mathcal{T}, b^{\prime}\right)$, of minimal possible length. Since $\Sigma \subseteq \Sigma^{\prime \prime},(\mathcal{X}, c)$ is also according to $\Sigma^{\prime}$, so by inflation condensation for $\Sigma^{\prime \prime}$, we have $b^{\prime \prime}=b^{\prime}$, as required.

\footnotetext{
${ }^{45}$ One can alternatively use an absoluteness argument like the proof of Claim 6; this argument does not use 6.4. Fix some trees $\mathcal{X}, \mathcal{V} \in V$ via $\Sigma$, and consider the tree of attempts to build trees $\mathcal{T}$ and $\mathcal{U}$ together with $\mathcal{T}$-cofinal branches $b \neq c$ and almost tree embeddings $\Pi_{b}:(\mathcal{T}, b) \hookrightarrow_{\text {alm }} \mathcal{X}$ and $\Pi_{c}:(\mathcal{T}, c) \hookrightarrow$ alm $\mathcal{U}$ and $\Pi: \mathcal{U} \hookrightarrow$ alm $\mathcal{V}$. Given objects of this form, then by 4.28 and strong hull condensation in $V,(\mathcal{T}, b)$ is via $\Sigma$, and $\mathcal{U}$ is via $\Sigma$, but therefore also $(\mathcal{T}, c)$ is via $\Sigma$, so $b=c$. So the tree is wellfounded, which suffices.
} 
This completes the proof of parts 4,5 and 7. Finally consider part 3:

Claim 11. Suppose $M$ is a premouse (not wcpm) and countable in $V$. Then $\Gamma$ has DJ iff $\Gamma^{\prime}$ has DJ in $V[G]$. Likewise for weak DJ with respect to $e$.

Proof. We just discuss DJ; weak DJ is almost the same.

If $\Gamma$ fails DJ then since $\Gamma \subseteq \Gamma^{\prime}$, clearly $\Gamma^{\prime}$ fails DJ in $V[G]$. So suppose $\Gamma$ has DJ, but $\Gamma^{\prime}$ does not in $V[G]$. Let $\mathcal{T} \in V[G]$ be a successor length tree according to $\Gamma^{\prime}$, witnessing this, via some $Q \unlhd M_{\infty}^{\mathcal{T}}$ and $\pi: M \rightarrow Q$.

Assume for now that $M$ has $\lambda$-indexing. Let $\mathcal{X} \in V$, via $\Gamma$, be a $\mathcal{T}$-terminallynon-dropping inflation of $\mathcal{T}$. Let $\sigma: M_{\infty}^{\mathcal{T}} \rightarrow M_{\infty}^{\mathcal{X}}$ be the final inflation copying map. So $\sigma$ is a near $\operatorname{deg}^{\mathcal{T}}(\infty)$-embedding, and by 4.50 , if $\mathcal{T}$ is terminally-nondropping then so is $\mathcal{X}$ and $\sigma \circ i^{\mathcal{T}}=i^{\mathcal{X}}$. So by considering $\sigma \circ i^{\mathcal{T}}$ and $\sigma(Q)$ if $Q \triangleleft M_{\infty}^{\mathcal{T}}$, we may in fact assume that $\mathcal{T} \in V$ is via $\Gamma$. But then since $M$ is countable in $V$, the existence of $\pi \in V[G]$ and absoluteness yields some $\pi^{\prime} \in V$ which gives a counterexample to DJ in $V$, contradiction.

Now suppose instead that $M$ has MS-indexing. Note by minimizing on $\operatorname{lh}(\mathcal{T})$, we get $\operatorname{lh}(\mathcal{T})<\Omega$ (for otherwise consider $\mathcal{T} \uparrow(\alpha+1)$ for sufficiently large $\alpha<\mathcal{T} \Omega$ ). Let $\widetilde{\mathcal{T}}$ be the tree according to $\Sigma^{\prime}$, corresponding to $\mathcal{T}$, so (by 2.12) $\left(M_{\infty}^{\widetilde{\mathcal{T}}}\right)^{\mathrm{pm}}=M_{\infty}^{\mathcal{T}}$ and if $M_{\infty}^{\mathcal{T}}$ is type 3 then $\mathrm{u}-\operatorname{deg}^{\widetilde{\mathcal{T}}}(\infty)=\operatorname{deg}^{\mathcal{T}}(\infty)+1>0$. Let $\widetilde{\mathcal{X}} \in V$, via $\Sigma$, be a $\mathcal{T}$-terminally-non-dropping inflation of $\widetilde{\mathcal{T}}$. Let

$$
\widetilde{\sigma}: M_{\infty}^{\widetilde{\mathcal{T}}} \rightarrow M_{\infty}^{\tilde{\mathcal{X}}}
$$

be the final copying map, so $\widetilde{\sigma}$ is a near u- $\operatorname{deg}^{\widetilde{\mathcal{T}}}(\infty)$-embedding. Let $\mathcal{X}$ be the tree according to $\Gamma$, corresponding to $\widetilde{\mathcal{X}}$. So $M_{\infty}^{\mathcal{X}}=\left(M_{\infty}^{\tilde{\mathcal{X}}}\right)^{\mathrm{pm}}$.

Now if $Q \triangleleft M_{\infty}^{\mathcal{T}}$ then note that either

$$
\tilde{\sigma}(Q) \triangleleft M_{\infty}^{\mathcal{X}} \text { or } \tilde{\sigma}(Q) \triangleleft \operatorname{Ult}\left(M_{\infty}^{\mathcal{X}} \mid\left(\mu^{+}\right)^{M_{\infty}^{\mathcal{X}}}, F\right)
$$

where $F=F\left(M_{\infty}^{\mathcal{X}}\right)$ and $\mu=\operatorname{cr}(F),{ }^{46}$ and so either from $(\mathcal{X}, \pi(Q), \widetilde{\sigma} \circ \pi)$ or

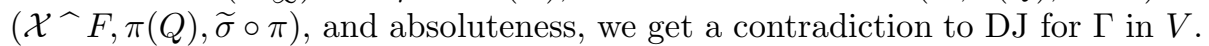

So suppose $Q=M_{\infty}^{\mathcal{T}}$. Because $\widetilde{\sigma}$ is a near $\mathrm{u}$ - $\operatorname{deg} \widetilde{\mathcal{T}}(\infty)$-embedding,

$$
\sigma=\tilde{\sigma} \uparrow\left(M_{\infty}^{\mathcal{T}}\right)^{\mathrm{sq}}:\left(M_{\infty}^{\mathcal{T}}\right)^{\mathrm{sq}} \rightarrow\left(M_{\infty}^{\mathcal{X}}\right)^{\mathrm{sq}}
$$

is a near $\operatorname{deg}^{\mathcal{T}}(\infty)$-embedding $M_{\infty}^{\mathcal{T}} \rightarrow M_{\infty}^{\mathcal{X}}$, and we have

$$
\mathrm{u}-\operatorname{deg} \tilde{\mathcal{X}}(\infty)=\mathrm{u}-\operatorname{deg}^{\widetilde{\mathcal{T}}}(\infty) \text { and } \operatorname{deg}^{\mathcal{X}}(\infty)=\operatorname{deg}^{\mathcal{T}}(\infty) \geq n
$$

If $\mathcal{T}$ drops in model on $b^{\mathcal{T}}$ then so do $\widetilde{\mathcal{T}}, \widetilde{\mathcal{X}}, \mathcal{X}$, and $\sigma \circ \pi: M \rightarrow M_{\infty}^{\mathcal{X}}$ is a near $\operatorname{deg}^{\mathcal{T}}(\infty)$-embedding, so by absoluteness we have a contradiction. So $\mathcal{T}$ does not drop in model, hence nor in degree, and likewise for $\mathcal{X}, \widetilde{\mathcal{T}}, \widetilde{\mathcal{X}}$. So $\tilde{\sigma} \circ i^{\widetilde{\mathcal{T}}}=i^{\tilde{\mathcal{X}}}$, so by $2.12, \sigma \circ i^{\mathcal{T}}=i^{\mathcal{X}}$. And $\pi(\alpha)<i^{\mathcal{T}}(\alpha)$ for some $\alpha \in \mathrm{OR}^{M}$, so $\sigma(\pi(\alpha))<\sigma\left(i^{\mathcal{T}}(\alpha)\right)=i^{\mathcal{X}}(\alpha)$, so again we have a contradiction.

This completes the proof of the theorem.

\footnotetext{
${ }^{46}$ Here if $Q \notin\left(M_{\infty}^{\mathcal{T}}\right)$ sq and $\widetilde{\sigma}\left(\nu\left(F\left(M_{\infty}^{\mathcal{T}}\right)\right)\right)>\nu\left(F\left(M_{\infty}^{\mathcal{X}}\right)\right)$ then we need to use $\mathcal{X}^{\wedge} F$ instead of just $\mathcal{X}$.
} 


\subsection{Universally Baire strategies}

The following corollaries on universally Baire representations for iteration strategies were motivated by related work of Steel. Given an iteration strategy $\Sigma$ on a countable premouse $M$, let $\widetilde{\Sigma}$ be the natural coding of $\Sigma\lceil H C$ over the reals. Note that without AC, it seems that the trees $S, T$ in the following corollary might not be trees on ordinals. However, the only non-ordinal information is specified by $\mathcal{X}=y(0)$. In Corollary 7.5 we prove a version where we do get trees $S, T$ on ordinals.

7.4 Corollary. Let $\Omega, \Gamma, M$ be as in Theorem 7.3, with $M$ countable. Then $\widetilde{\Gamma}\left\lceil\mathbb{R}\right.$ is $\Omega$-universally Baire. In fact, there are trees $S, T$ on $\omega \times \mathcal{H}_{\Omega}$ such that letting $G$ be $V$-generic for $\operatorname{Col}(\omega,<\Omega)$, then $S, T$ project to complements in $V[G]$, and

$$
p[T]^{V[G]}=\widetilde{\Gamma^{\prime}} \uparrow \mathbb{R},
$$

where $\Gamma^{\prime}$ is the extension of $\Gamma$ given by Theorem 7.3.

Proof. Let $\Sigma$ be the u-strategy corresponding to $\Gamma$, as in Theorem 7.3.

Let $T$ be the tree of attempts to build $\left(x^{\prime},(x, y)\right)$, where $x, x^{\prime} \in{ }^{\omega} \omega, x$ codes a pair $(\mathcal{T}, b)$, where $\mathcal{T}$ is a (potential) countable limit length $\mathrm{u}-m$-maximal tree on $M$ and $b$ is a $\mathcal{T}$-cofinal branch, $x^{\prime}$ codes the corresponding (potential) $m$ maximal tree $\left(\mathcal{T}^{\prime}, b^{\prime}\right)$, and $y \in{ }^{\omega}\left(\mathcal{H}_{\Omega}\right)$ specifies $y(0)=\mathcal{X}$ is some tree on $M$ via $\Sigma$, of length $<\Omega$, and $y$ codes an almost tree embedding $\Pi:(\mathcal{T}, b) \hookrightarrow_{\text {alm }} \mathcal{X}$.

Let $S$ be natural tree for the complement. That is, $S$ builds $\left(\widetilde{x^{\prime}},(\widetilde{x}, \widetilde{y})\right)$ such that either $\widetilde{x^{\prime}}$ codes garbage information, or $\widetilde{x}, \widetilde{x^{\prime}}$ code $(\mathcal{T}, \widetilde{b}),\left(\mathcal{T}^{\prime}, \widetilde{b^{\prime}}\right)$ as above, and $\widetilde{y}$ codes a tuple $\left(x^{\prime},(x, y)\right) \in[T]$, and $x^{\prime}$ codes the pair $\left(\mathcal{T}^{\prime}, c\right)$ with $c \neq \widetilde{b^{\prime}}$.

Now $\operatorname{Col}(\omega,<\Omega)$ is $\Omega$-cc, so Theorem 7.3 applies. But clearly by strong hull condensation we have $p[S] \cap p[T]=\emptyset$ in both $V$ and $V[G]$. ${ }^{47}$ And by the proof of 7.3 , note that $p[T]^{V[G]}=\widetilde{\Gamma^{\prime}} \uparrow \mathbb{R}$, and $S, T$ project to complements in $V[G]$.

If $\Omega$ is inaccessible, we can improve the conclusion; in the following proof, the trees we form are analogous to those formed by direct limits of mice used by Steel.

7.5 Corollary. Adopt the hypotheses and notation of Corollary 7.4. Suppose also that for no $\alpha<\Omega$ is $\Omega$ the surjective image of $\mathcal{P}(\alpha)$. Then there are trees $S, T \in \mathrm{OD}_{\Gamma, M}$ witnessing Corollary 7.4 with $S, T$ on $\omega \times \Omega$.

Proof. Let $\Sigma$ be as before. By the proof of 7.4, it suffices to show that for each $\mathcal{T}$ via $\Sigma$ of length $<\Omega$, there is some $\mathcal{X} \in \mathrm{OD}_{\Gamma, M}$ such that $\mathcal{X}$ is via $\Sigma$, of length $<\Omega$, and is a $\mathcal{T}$-terminally-non-dropping inflation of $\mathcal{T}$. For by the largeness assumption of $\Omega$ (including regularity), we can enumerate all such $\mathcal{X}$ in ordertype $\Omega^{48}$ in an $\mathrm{OD}_{\Gamma, M}$ fashion, leading to an $\mathrm{OD}_{\Gamma, M}$ tree $T$ on $\omega \times \Omega$.

\footnotetext{
${ }^{47}$ If $\mathcal{H}_{\Omega}$ is not wellordered in $V$, then we can't quite use the usual argument here to deduce that $V[G] \models " p[T] \cap p[S]=\emptyset "$, given that $V \models " p[T] \cap p[S]=\emptyset "$, However, one could note that for any given tree $\mathcal{X}$ as a choice of $y(0)$, the sub-trees $S_{\mathcal{X}}$ and $T_{\mathcal{X}}$ can be taken on ordinals. So if $V[G] \models " p[T] \cap p[S] \neq \emptyset "$, then we could fix a specific $\mathcal{X}$ and $\mathcal{Y}$ with $V[G] \models " p\left[T_{\mathcal{X}}\right] \cap p\left[S_{\mathcal{Y}}\right] \neq \emptyset "$ and deduce that $V \models " p\left[\mathcal{T}_{\mathcal{X}}\right] \cap p\left[S_{\mathcal{Y}}\right] \neq \emptyset$ ", a contradiction.

${ }^{48}$ Enumerate those of length $\alpha$ before those of length $\beta$, when $\alpha<\beta$.
} 
So fix $\chi<\Omega$ and let $\mathscr{T}$ be the set of all trees via $\Sigma$ of length $<\chi$. We define $\lambda<\Omega$ and a partition $\overrightarrow{\mathscr{T}}=\left\langle\mathscr{T}_{\alpha}\right\rangle_{\alpha<\lambda}$ of $\mathscr{T}$ and a sequence $\overrightarrow{\mathcal{X}}=\left\langle\mathcal{X}_{\alpha}\right\rangle_{\alpha<\lambda}$ of trees $\mathcal{X}_{\alpha}$ via $\Sigma$, such that for each $\alpha<\lambda$, we have: (i) $\overrightarrow{\mathscr{T}}, \overrightarrow{\mathcal{X}}$ are $\mathrm{OD}_{\Gamma, M}$, (ii) $\mathscr{T}_{\alpha} \neq \emptyset$, (iii) $\ln \left(\mathcal{X}_{\alpha}\right)<\Omega$, and (iv) $\mathcal{X}_{\alpha}$ is a $\mathcal{T}$-terminally-non-dropping inflation of each $\mathcal{T} \in \mathscr{T}_{\alpha}$. Clearly this suffices.

So suppose we have defined $\left\langle\mathscr{T}_{\alpha}\right\rangle_{\alpha<\eta}$ and $\left\langle\mathcal{X}_{\alpha}\right\rangle_{\alpha<\eta}$ satisfying the requirements so far, and suppose that $\mathscr{T}^{\prime}=\mathscr{T} \backslash \bigcup_{\alpha<\eta} \mathscr{T}_{\alpha} \neq \emptyset$; otherwise we are done.

We set $\mathcal{X}_{\eta}$ to be the minimal simultaneous inflation of $\mathscr{T}^{\prime}$. This exists and has length $<\Omega$. For otherwise via minimal simultaneous inflation, we reach a tree $\mathcal{X}$ of length $\Omega+1$. Each extender used along $[0, \Omega)_{\mathcal{X}}$ is copied from some $\mathcal{T} \in \mathscr{T}^{\prime}$. But each $\mathcal{T} \in \mathscr{T}^{\prime}$ has length $<\Omega$, and since $\Omega$ is regular, it follows that there is $\alpha_{\mathcal{T}}<{ }^{\mathcal{X}} \Omega$ such that no extender used in $\left(\alpha_{\mathcal{T}}, \Omega\right]_{\mathcal{X}}$ is copied from $\mathcal{T}$. But then $\mathcal{T} \mapsto \alpha_{\mathcal{T}}$ is cofinal in $\Omega$, and since $\Omega$ is regular, this gives a surjection $\mathcal{P}(\alpha) \rightarrow \Omega$, a contradiction.

Now by 5.2 , there is some $\mathcal{T} \in \mathscr{T}^{\prime}$ such that $\mathcal{X}_{\eta}$ is $\mathcal{T}$-terminally-nondropping. So letting $\mathscr{T}_{\eta}$ be the set of all such $\mathcal{T}$, we are done.

\subsection{Restricting weak Dodd-Jensen strategies from $V[G]$}

The proof of the following corollary involves standard comparison of premice, and the author does not see a version for wcpms. We will prove an extension of the corollary in $\$ 10$, once we have Theorem 9.1 at our disposal. Recall that strategies with weak DJ with respect to a given enumeration $e$ (in ordertype $\omega$ ) are unique; see Remark 4.46.

7.6 Corollary. Let $\Omega>\omega$ be regular. Let $M$ be a countable $m$-sound premouse. Let $e$ be an enumeration of $M$ in ordertype $\omega$. Let $\mathbb{P}$ be an $\Omega$-cc forcing and $G$ be $V$-generic for $\mathbb{P}$. Then:

1. $V \models$ "There is an $(m, \Omega+1)$-strategy for $M$ with weak $D J$ with respect to $e$ " iff $V[G]$ satisfies the same statement.

2. If $\Sigma$ is an (hence the unique) $(m, \Omega+1)$-strategy for $M$ with weak $D J$ with respect to $e$, and $\Sigma^{\prime}$ likewise in $V[G]$, then $\Sigma \subseteq \Sigma^{\prime}$.

Proof. The forward direction and the fact that $\Sigma \subseteq \Sigma^{\prime}$ is by Theorem 7.3.

So suppose that in $V[G], \Sigma^{\prime}$ is an $(m, \Omega+1)$-strategy for $M$ with weak DJ with respect to $e \in V$. Let $p_{0} \in \mathbb{P}$ force this fact. Let $\Sigma=\Sigma^{\prime} \uparrow V$. It suffices to see that $\Sigma \in V$, as then $\Sigma$ has weak DJ with respect to $e$ in $V$, and part 2 follows from the uniqueness of this strategy (see Remark 4.46).

So let $\mathcal{T} \in V$ be of limit length via $\Sigma^{\prime}$ (thus, $\operatorname{lh}(\mathcal{T}) \leq \Omega$ ), and $b=\Sigma^{\prime}(\mathcal{T})$. Let $\dot{\Sigma}^{\prime}, \dot{b}$ be names for $\Sigma^{\prime}, b$. By the following claim, $b \in V$ and $p_{0}$ forces " $b=\dot{\Sigma}^{\prime}(\mathcal{T})$ ", which clearly suffices.

Claim 1. For each $\alpha<\operatorname{lh}(\mathcal{T}), p_{0}$ decides the truth of " $\alpha \in \dot{b}$ ".

Proof. Suppose not. We form a Boolean-valued comparison of generic phalanxes $\Phi(\mathcal{T}, \dot{b})$. Inductively on stages $\alpha \leq \Omega$, we define a monotone increasing sequence $\left\langle\xi_{\alpha}\right\rangle_{\alpha<\Omega}$ of ordinals and a sequence $\left\langle N_{\alpha}\right\rangle_{\alpha \leq \Omega}$ of premice (in $V$ ) and a sequence 
$\left\langle\dot{\mathcal{T}}_{\alpha}\right\rangle_{\alpha \leq \Omega}$ of names for padded iteration trees on $M$. In fact, $\dot{\mathcal{T}}_{\alpha}$ is just the name for the padded tree via $\dot{\Sigma}^{\prime}$, extending $(\mathcal{T}, \dot{b})$, of length $\operatorname{lh}(\mathcal{T})+\alpha+1$, which uses extenders with indices $\left\langle\xi_{\beta}\right\rangle_{\beta<\alpha}$ (where we pad when there is no extender indexed at $\left.\xi_{\beta}\right)^{49}$, and $p_{0}$ will force that $N_{\alpha}=M_{\infty}^{\dot{\mathcal{T}}_{\alpha}} \| \xi_{\alpha}$. Given a $\mathbb{P}$-name $\sigma$, we write $\sigma^{0}$ for the $\mathbb{P} \times \mathbb{P}$-name for $\sigma^{\dot{G}_{0}}$, where $\dot{G}_{0}$ is the $\mathbb{P} \times \mathbb{P}$-name for the projection of the $\mathbb{P} \times \mathbb{P}$-generic on the left coordinate; likewise for $\sigma^{1}$ and the right coordinate.

We begin with $\dot{\mathcal{T}}_{0}=(\check{\mathcal{T}}, \dot{b})$.

Given $\dot{\mathcal{T}}_{\alpha}$, where $\alpha<\Omega$, let $\xi_{\alpha}$ be the least ordinal $\xi$ such that $p_{0}$ forces " $\xi \leq \mathrm{OR}\left(M_{\infty}^{\dot{\mathcal{T}}_{\alpha}}\right)$ " and for some $p, q \leq p_{0}$, we have

$$
(p, q) \models_{\mathbb{P} \times \mathbb{P}} " M_{\infty}^{\dot{\mathcal{T}}_{\alpha}^{0}}\left|\xi \neq M_{\infty}^{\dot{\mathcal{T}}_{\alpha}^{1}}\right| \xi ",
$$

if there is such a $\xi$; otherwise $\xi_{\alpha}$ is undefined and we stop the construction. Assuming $\xi_{\alpha}$ is defined, this determines $\dot{\mathcal{T}}_{\alpha+1}$, and note that $p_{0}$ decides the value of $N_{\alpha}={ }_{\text {def }} M_{\alpha}^{\dot{\mathcal{T}}_{\infty}} \| \xi_{\alpha}$.

Given $\dot{\mathcal{T}}_{\alpha}$ for all $\alpha<\eta$, for a limit $\eta$, note that $\dot{\mathcal{T}}_{\eta}$ is determined.

Clearly $\xi_{\alpha} \leq \xi_{\beta}$ for $\alpha<\beta$ (with $\xi_{\alpha}=\xi_{\beta}$ only if $\beta=\alpha+1$ and we are using MS-indexing and the usual superstrong/type 2 situation occurs).

SubClaim 1.1. $p_{0}$ forces " $\xi_{\alpha}$ exists for every $\alpha<\Omega$ ".

Proof. Suppose not and let $\alpha$ be least such. Write $\dot{\mathcal{U}}=\dot{\mathcal{T}}_{\infty}$. Let $\xi$ be least such that for some $q \leq p_{0}, q$ forces " $\xi=\operatorname{OR}\left(M_{\infty}^{\dot{\mathcal{U}}}\right)$ ". Note that $p_{0}$ determines $N={ }_{\text {def }} M_{\infty}^{\dot{\mathcal{U}}} \mid \xi$ (so $N \in V$ ) and $q$ forces " $M_{\infty}^{\dot{\mathcal{U}}}=N$ ".

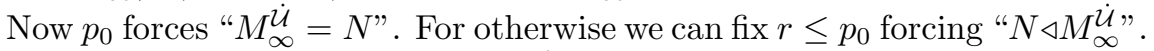
Then $N$ is fully sound, so $q$ forces " $b \dot{\mathcal{U}}$ does not drop in model or degree, so $i^{\dot{\mathcal{U}}}: M \rightarrow N$ exists and is an $m$-embedding", so by absoluteness, $r$ forces "There is an $m$ embedding $\pi: M \rightarrow N$ ", which contradicts weak DJ below $r$.

Similar considerations using weak DJ now also give that either

(i) $p_{0}$ forces " $b \dot{\mathcal{U}}$ drops in model or degree", or

(ii) $p_{0}$ forces " $b$ u does not drop in model or degree",

and moreover, if (ii) holds then there is $\pi \in V$ such that $p_{0}$ forces " $i \dot{\mathcal{U}}=\pi$ ", and of course if (ii) holds then letting $\pi: \mathfrak{C}_{n+1}(N) \rightarrow N$ be the core map, where $n<\omega$ is largest such that $N$ is $n$-sound, then $p_{0}$ forces "there is $\beta$ such that $\beta+1 \in b^{\dot{\mathcal{U}}}$ and $i_{\beta+1, \infty}^{* \dot{\mathcal{U}}}=\pi^{\prime \prime}$.

We can now recover the sequence of extenders $E$ forced to be used in $\dot{\mathcal{U}}$ to form $\pi$, in the usual manner (cf. [22, 4.3 and Remark]). (So this sequence is in $V$.) But by the Zipper Lemma, $p_{0}$ forces that there is some such $E$ which is not used in $\mathcal{T}$. Let $E$ be the least such.

\footnotetext{
${ }^{49}$ We are using the conventional algorithm for comparison by least disagreement, not modified as in [20] or [18].
} 
By the rules of comparison, there cannot be a single $\gamma$ such that $p_{0}$ forces " $E_{\gamma}^{\dot{\mathcal{U}}}=E$ ", but since ind $(E)$ is fixed, therefore $M$ is MS-indexed, $E$ is type 2 and there is $\gamma$ such that $p_{0}$ forces "either

(a) $E_{\gamma}^{\dot{\mathcal{U}}}=E$ and $E_{\gamma+1}^{\dot{\mathcal{U}}}=\emptyset$, or

(b) $E_{\gamma}^{\dot{\mathcal{U}}}$ is superstrong and $E_{\gamma+1}^{\dot{\mathcal{U}}}=E=F\left(M_{\gamma+1}^{\dot{\mathcal{U}}}\right)$ and $\operatorname{cr}\left(E_{\gamma}^{\dot{\mathcal{U}}}\right)=\operatorname{lgcd}\left(M_{\gamma+1}^{* \dot{\mathcal{U}}}\right)$ ", and moreover both options get forced by some condition $\leq p_{0}$.

Note that $P=M_{\gamma+1}^{\dot{\mathcal{U}}}$ of option (b) is in $V$, and $P$ is non-sound, so in option (a), $P=M_{\gamma}^{\dot{\mathcal{u}}}$. But now arguing like we $\operatorname{did}$ for $N, \pi$ and the sequence of extenders above, it follows that there is a superstrong extender $F \in V$ such that $p_{0}$ forces that $F$ is used on the branch leading to $P$, but then in fact $p_{0}$ forces " $E_{\gamma}^{\dot{\mathcal{U}}}=F$ and $E_{\gamma+1}^{\dot{\mathcal{U}}}=E$ " a contradiction.

By the subclaim, we reach $\dot{\mathcal{U}}={ }_{\operatorname{def}} \dot{\mathcal{T}}_{\Omega}$, of length $\Omega+1$. Let $\dot{c}=b^{\dot{\mathcal{U}}}$. Since $\mathbb{P}$ is $\Omega$-cc, we get a club $B \subseteq \Omega$ such that $p_{0}$ forces " $B \subseteq \dot{c}$ and $[\alpha, \Omega)_{\dot{\mathcal{U}}}$ does not drop and $i_{\alpha \Omega}^{\dot{\mathcal{U}}}(\alpha)=\Omega$ for each $\alpha \in B$ ". Let $N=\operatorname{stack}_{\alpha<\Omega} N_{\alpha}$. Note $p_{0}$ forces that $N=M(\dot{\mathcal{U}})$ and $M_{\alpha}^{\dot{\mathcal{U}}} \|\left(\alpha^{+}\right)^{M_{\alpha}^{\dot{\mathcal{u}}}}=N \mid\left(\alpha^{+}\right)^{N}$ for each $\alpha \in B$.

We will now run a version of the standard proof of termination of comparison. We first define a strictly increasing sequence $\left\langle\alpha_{n}\right\rangle_{n<\omega} \subseteq B$ and a $\subseteq$-increasing sequence $\left\langle X_{n}\right\rangle_{n<\omega}$ with $X_{n} \subseteq \Omega^{+}$and $\operatorname{card}\left(X_{n}\right)<\Omega$.

Let $X_{0}=\emptyset$ and $\alpha_{0}=\min (B)$. Suppose we have $X_{n}, \alpha_{n}$, and

$$
p_{0} \Vdash \bullet X_{n} \cap\left(\Omega^{+}\right)^{M_{\Omega}^{\dot{u}}} \subseteq i_{\alpha_{n} \Omega}^{\dot{\mathcal{u}}}\left[\left(\alpha_{n}^{+}\right)^{N}\right] " .
$$

Let $X_{n+1}=X_{n} \cup\left\{\beta<\Omega^{+} \mid \exists q \leq p_{0}\right.$ s.t. $q$ forces " $\beta \in i_{\alpha_{n} \Omega}^{\dot{\mathcal{u}}}\left[\left(\alpha_{n}^{+}\right)^{N}\right]$ " $\}$. Clearly then $X_{n+1} \subseteq \Omega^{+}, \operatorname{card}\left(X_{n+1}\right)<\Omega$ by the $\Omega$-cc and since $\left(\alpha_{n}^{+}\right)^{N}<\Omega$, and

$$
p_{0} \Vdash{ }^{i} i_{\alpha_{n} \Omega}^{\dot{\mathcal{U}}}\left[\left(\alpha_{n}^{+}\right)^{N}\right] \subseteq X_{n+1} \cap\left(\Omega^{+}\right)^{M_{\Omega}^{\dot{u}}} " .
$$

Now let $\alpha_{n+1}$ be the least $\alpha \in B$ such that $\alpha>\alpha_{n}$ and

$$
p_{0} \Vdash " X_{n+1} \cap\left(\Omega^{+}\right)^{M_{\Omega}^{\dot{u}}} \subseteq i_{\alpha \Omega}^{\dot{\mathcal{u}}}\left[\left(\alpha^{+}\right)^{N}\right] .
$$

By the $\Omega$-cc and since $\operatorname{card}\left(X_{n+1}\right)<\Omega, \alpha_{n+1}$ exists.

Now let $\alpha=\sup _{n<\omega} \alpha_{n}$ and $X=\bigcup_{n<\omega} X_{n}$. So $\alpha \in B$, and note that

$$
p_{0} \Vdash \vdash " X \cap\left(\Omega^{+}\right)^{M_{\Omega}^{\dot{u}}}=i_{\alpha \Omega}^{\dot{u}}\left[\left(\alpha^{+}\right)^{N}\right] " .
$$

So $p_{0} \Vdash \models$ " $X \cap\left(\Omega^{+}\right)^{M_{\Omega}^{\dot{u}}}$ is cofinal in $\left(\Omega^{+}\right)^{M_{\Omega}^{\dot{u}}}$ and has ordertype $\left(\alpha^{+}\right)^{N "}$. It follows that $p_{0}$ decides the value of $\left(\Omega^{+}\right)^{M_{\Omega}^{\dot{u}}}$, and decides $i_{\alpha \Omega}^{\dot{\mathcal{U}}}\left\lceil\left(\alpha^{+}\right)^{N}\right.$.

Now repeat the preceding construction, starting with $\alpha_{0}^{\prime}>\alpha$, and producing a limit $\alpha^{\prime}$. Then note that $p_{0}$ decides $i_{\alpha \alpha^{\prime}}^{\dot{U}} \uparrow\left(\alpha^{+}\right)^{N}$. But $p_{0}$ decides $N \mid\left(\left(\alpha^{\prime}\right)^{+}\right)^{N}$, and hence decides $i_{\alpha \alpha}^{\dot{\mathcal{U}}} \uparrow\left(N \mid\left(\alpha^{+}\right)^{N}\right)$ (not just the restriction to the ordinals; one needs to know that the codomains match before being able to deduce the agreement of the embeddings). This contradicts comparison much as before, proving the claim. 
This completes the proof of the corollary.

7.7 Corollary. Let $\Omega>\omega$ be regular. Let $G$ be $V$-generic for an $\Omega$-cc forcing. Let $M \in V[G]$ be an $\omega$-sound premouse with $\rho_{\omega}^{M}=\omega$. Then:

- $V[G] \models$ " $M$ is $(\omega, \Omega+1)$-iterable" iff $M \in V$ and $M$ is $(\omega, \Omega+1)$-iterable.

- If $\Sigma$ is an (hence the unique) ( $\omega, \Omega+1)$-strategy for $M$, and $\Sigma^{\prime}$ likewise in $V[G]$, then $\Sigma \subseteq \Sigma^{\prime}$.

Proof. Recall first that for an $\omega$-sound premouse $N$ with $\rho_{\omega}^{N}=\omega$, if $\Sigma$ is an $(\omega, \Omega+1)$-strategy for $N$, then $\Sigma$ has DJ, and hence, weak DJ with respect to any enumeration $e$ of $N$, and hence, strong hull condensation, by Theorem 4.47. So if $M \in V$, then the conclusions of the theorem follow from Theorem 7.3 and Corollary 7.6. So we only need to see that if $M$ is $(\omega, \Omega+1)$-iterable in $V[G]$ then $M \in V$. Suppose not. Let $\dot{M}$ be a name for $M$ and $p_{0}$ force the facts we have about $M$, and letting $\lambda=\mathrm{OR}^{M}$, such that $p_{0}$ forces "OR $\dot{M}=\lambda$ ". Then $p_{0}$ decides $\mathbb{E}_{+}^{M}$, so $M \in V$. For if not then we can form a Boolean-valued comparison of generic interpretations of $\dot{M}$, below $p_{0}$. This is almost the same as the proof of Corollary 7.6, and leads to contradiction as there. We leave the details to the reader.

In Corollaries 10.12 and 10.13, later in the paper, when we have some more results at our disposal, we will be able to deduce further generic absoluteness results under some choice assumptions.

7.8 Question. One can of course ask to what extent other types of forcings preserve iterability. Schindler and the author have found a couple of interesting counterexamples, which are yet to appear; these include a model of ZFC+ " $M_{1}^{\#}$ exists and is $\left(\omega, \omega_{1}+1\right)$-iterable, but there is a $\sigma$-distributive forcing which forces that $\check{M}_{1}^{\#}$ is not $\left(\omega, \omega_{1}+1\right)$-iterable".

The following questions, for example, seem to be open. Let $V[G]$ be an $\omega$-closed forcing extension of $V$. Is every $\omega$-mouse of $V$ also an $\omega$-mouse of $V[G]$ ? Is every $\omega$-mouse of $V[G]$ also an $\omega$-mouse of $V$, or at least, $\omega_{1}$-iterable in $V$ ? (Clearly every $\omega_{1}$-iterable premouse of $V$ is also $\omega_{1}$-iterable in $V[G]$.) $\operatorname{Can} \operatorname{Col}\left(\omega_{1}, \kappa\right)$, for some $\kappa \geq \omega_{1}$, consistently kill the $\left(\omega, \omega_{1}+1\right)$-iterability of $M_{1}^{\#}$ ?

7.9 Remark. Let $\Omega$ be regular uncountable and $M$ be an $(\omega, \Omega+1)$-iterable $\omega$-mouse, as witnessed by $\Sigma$, such that $M$ has 2 measurable cardinals, and suppose that $\mathbb{P}$ is not $\Omega$-cc. Then the method of extending $\Sigma$ to $V[G]$ used for $\Omega$-cc forcing, fails for $\mathbb{P}$. For let $\left\langle p_{\alpha}\right\rangle_{\alpha<\Omega} \subseteq \mathbb{P}$ be an antichain. Let $\mu_{0}<\mu_{1}$ be measurables of $M$. Define the $\mathbb{P}$-name $\dot{\mathcal{T}}$ where below $p_{\alpha}, \dot{\mathcal{T}}$ is the length $\alpha$ linear iteration of $M$ using a measure on $\mu_{0}$ and its images, followed by a measure on the image of $\mu_{1}$. Letting $\mathcal{X}$ be the minimal simultaneous inflation of the $\mathcal{\mathcal { T }}$ through length $\Omega+1$, clearly $\mathcal{X}$ is just the length $\Omega$ linear iteration of $M$ at $\mu_{0}$. So the process is forced to fail. 


\section{The factor tree $\mathcal{X} / \mathcal{T}$}

In this section we give a second perspective on inflation $\mathcal{T} \rightsquigarrow \mathcal{X}$, in which we shift the focus from the $\mathcal{T}$-copied extenders to the $\mathcal{T}$-inflationary extenders. From this perspective, a natural analogy arises: $\mathcal{X}$ induces what can be considered an iteration tree $\widetilde{\mathcal{X}}$ on $\mathcal{T}$, which consists of a sequence of (standard) iteration trees $\mathcal{X}^{\alpha}$ on $M$ (instead of a sequence of models) and whose extenders are just the $\mathcal{T}$-inflationary extenders of $\mathcal{X}$. We will also define various tree embeddings $\Pi^{\alpha \beta}: \mathcal{X}^{\alpha} \hookrightarrow \mathcal{X}^{\beta}$, in the right circumstances, analogous to iteration maps, and introduce more bookkeeping. Benjamin Siskind has recently (in 2018) developed this perspective formally, proving versions of the Shift Lemma and so forth in this context. (We do not use any of Siskind's work here, however.)

\subsection{The factor tree order $<^{\mathcal{X} / \mathcal{T}}$}

We begin by describing an iteration tree order $<^{\mathcal{X}} / \mathcal{T}$ determined by an inflation $\mathcal{T} \rightsquigarrow \mathcal{X}$. When we reduce an (appropriate) stack of two normal trees $(\mathcal{T}, \mathcal{U})$ to a single normal tree $\mathcal{X}$ (via an iteration strategy with inflation condensation), then $\mathcal{X}$ will be an inflation of $\mathcal{T}$, and $\mathcal{U}$ will have tree order $<^{\mathcal{U}}=<^{\mathcal{X}} / \mathcal{T}$.

8.1 Definition. Let $\mathcal{X}$ be an inflation of $\mathcal{T}$ and $t=t^{\mathcal{T} \rightsquigarrow \mathcal{X}}$.

If $\alpha<\operatorname{lh}(\mathcal{X})$ then the $\mathcal{T}$-unravelling of $\mathcal{X} \vdash(\alpha+1)$, if it exists, is the unique non- $\mathcal{T}$-pending inflation $\mathcal{W}$ of $\mathcal{T}$ such that $\mathcal{W}$ extends $\mathcal{X} \uparrow(\alpha+1)$ and $t^{\mathcal{T} \rightsquigarrow \mathcal{W}}(\beta)=0$ for every $\beta \geq \alpha$. Existence just depends on the models being wellfounded. We say that $\mathcal{X}$ is $\mathcal{T}$-good (or just good) iff the $\mathcal{T}$-unravelling of $\mathcal{X} \uparrow(\alpha+1)$ exists for every $\alpha<\ln (\mathcal{X})$.

8.2 Definition. Let $\mathcal{X}$ be a good inflation of $\mathcal{T}$ and $t=t^{\mathcal{T} \rightsquigarrow \mathcal{X}}$. Let $\left\langle\lambda^{\alpha}\right\rangle_{\alpha<\iota}$ enumerate in increasing order all $\lambda<\operatorname{lh}(\mathcal{X})$ such that either $\lambda=0$, or $\lambda=\zeta+1$ where $t(\zeta)=1$, or $\lambda$ is a limit of such ordinals. If $\alpha+1<\iota$ then let $\zeta^{\alpha}+1=\lambda^{\alpha+1}$ and $L^{\alpha}=\left[\lambda^{\alpha}, \zeta^{\alpha}\right]$, and if $\alpha+1=\iota$ then let $L^{\alpha}=\left[\lambda^{\alpha}, \ln (\mathcal{X})\right)$. So the intervals $L^{\alpha}$ are disjoint and partition $[0, \ln (\mathcal{X}))$. For $\delta<\operatorname{lh}(\mathcal{X})$ let $\eta_{\delta}$ be the $\eta<\iota$ such that $\delta \in L^{\eta}$.

We write $\mathcal{X}^{\alpha}$ for the $\mathcal{T}$-unravelling of $\mathcal{X}\left\lceil\left(\lambda^{\alpha}+1\right)\right.$, with associated objects $\left(t^{\alpha}, C^{\alpha}, \ldots\right)$. If $\lambda^{\alpha} \in C^{\alpha}$ then also let $\theta^{\alpha}=f^{\alpha}\left(\lambda^{\alpha}\right)$; otherwise $\theta^{\alpha}$ is not defined. Then either

$-\lambda^{\alpha} \notin C^{\alpha}$ and $\operatorname{lh}\left(\mathcal{X}^{\alpha}\right)=\lambda^{\alpha}+1$, or

$-\lambda^{\alpha} \in C^{\alpha}$ and $\operatorname{lh}\left(\mathcal{X}^{\alpha}\right)=\lambda^{\alpha}+\left(\operatorname{lh}(\mathcal{T})-\theta^{\alpha}\right)$.

Let $\left(\lambda^{\alpha}, \zeta^{\alpha}, L^{\alpha}, \mathcal{X}^{\alpha}, t^{\alpha}, \ldots\right)^{\mathcal{T} \rightsquigarrow \mathcal{X}}={ }_{\operatorname{def}}\left(\lambda^{\alpha}, \zeta^{\alpha}, L^{\alpha}, \mathcal{X}^{\alpha}, t^{\alpha}, \ldots\right)$. If $\lambda^{\alpha} \in C^{\alpha}$, then for $\xi<\operatorname{lh}(\mathcal{T})$ we set

$$
\begin{aligned}
& \left(I_{\xi}^{\alpha}\right)^{\mathcal{T} \rightsquigarrow \mathcal{X}}={ }_{\operatorname{def}} \lim _{\lambda \rightarrow \operatorname{lh}\left(\mathcal{X}^{\alpha}\right)} I_{\lambda ; \xi}^{\mathcal{T} \rightsquigarrow \mathcal{X}^{\alpha}}, \\
& \left(\pi_{\xi i}^{\alpha}\right)^{\mathcal{T} \rightsquigarrow \mathcal{X}}={ }_{\operatorname{def}} \lim _{\lambda \rightarrow \operatorname{lh}\left(\mathcal{X}^{\alpha}\right)} \pi_{\lambda ; \xi i}^{\mathcal{T} \rightsquigarrow \mathcal{X}^{\alpha}},
\end{aligned}
$$


etc. Note here that because $\mathcal{X}^{\alpha} \uparrow\left[\lambda^{\alpha}, \operatorname{lh}\left(\mathcal{X}^{\alpha}\right)\right)$ is formed by copying, this makes sense and $\left(I_{\xi}^{\alpha}\right)^{\mathcal{T} \rightsquigarrow \mathcal{X}}=I_{\lambda ; \xi}^{\mathcal{T} \rightsquigarrow \mathcal{X}^{\alpha}}$, etc, for all sufficiently large $\lambda<\operatorname{lh}\left(\mathcal{X}^{\alpha}\right)$. Of course if $\operatorname{lh}(\mathcal{T})$ is a successor, then $\operatorname{lh}\left(\mathcal{X}^{\alpha}\right)$ is a successor $\lambda+1$ and $\left(I_{\xi}^{\alpha}\right)^{\mathcal{T} \rightsquigarrow \mathcal{X}}=$ $I_{\lambda ; \xi}^{\mathcal{T} \rightsquigarrow \mathcal{X}^{\alpha}}$, etc; this is the main case of interest.

8.3 Definition. Let $\mathcal{X}$ be a good inflation of $\mathcal{T}$. Adopt notation as in 8.2. Then $<^{\mathcal{X} / \mathcal{T}}$ denotes the order on $\iota$ defined recursively as follows: for each $\alpha<\iota$,

$$
[0, \alpha)_{\mathcal{X} / \mathcal{T}}=\bigcup_{\delta<\mathcal{X}_{\lambda^{\alpha}}}\left[0, \eta_{\delta}\right]_{\mathcal{X} / \mathcal{T}}
$$

We remark that $\left\{\eta_{\delta} \mid \delta<^{\mathcal{X}} \lambda^{\alpha}\right\}$ need not be closed downward under $<^{\mathcal{X} / \mathcal{T}}$. We will verify soon that $<^{\mathcal{X} / \mathcal{T}}$ is an iteration tree order, but first we have the following approximation:

8.4 Lemma. Let $\mathcal{X}$ be a good inflation of $\mathcal{T}$. Adopt notation as above. Let $b_{\alpha}=[0, \alpha)_{\mathcal{X} / \mathcal{T}}$. Then (i) $<\mathcal{X} / \mathcal{T}$ is transitive, (ii) $b_{\alpha} \subseteq \alpha$, (iii) if $\alpha=\gamma+1$ then $\eta=\max \left(b_{\alpha}\right)$ and $[0, \eta]_{\mathcal{X} / \mathcal{T}} \subseteq b_{\alpha}$ where $\eta$ is least such that $\operatorname{cr}\left(E_{\zeta^{\gamma}}^{\mathcal{X}}\right)<\iota\left(\operatorname{ex}_{\zeta^{\eta}}^{\mathcal{X}}\right)$, 50 and (iv) if $\alpha$ is a limit then $b_{\alpha}$ is cofinal in $\alpha$.

Proof. Transitivity is a straightforward induction, and the other facts follow easily from the definitions.

8.5 Definition. For an iteration tree $\mathcal{V}$ and $\alpha<\operatorname{lh}(\mathcal{V})$, let $\mathcal{V} \geq \alpha=\mathcal{V} \uparrow[\alpha, \operatorname{lh}(\mathcal{V}))$, considered as an iteration tree on the phalanx $\Phi(\mathcal{V}\lceil\alpha+1)$. Given an iteration tree order $<_{0}$ and $\alpha<\operatorname{lh}\left(<_{0}\right)$, let $<_{0}^{(\alpha)}=<_{0} \uparrow\left\{\delta \mid \delta \geq_{0} \alpha\right\}$.

8.6 Lemma. Let $\mathcal{X}$ be a good inflation of $\mathcal{T}$. Adopt notation as above. Let $\alpha<\operatorname{lh}(\mathcal{X} / \mathcal{T})$. Then:

$-\lambda^{\alpha} \in\left(C^{-}\right)^{\alpha}$ iff $\lambda^{\alpha}+1<\ln \left(\mathcal{X}^{\alpha}\right)$.

Suppose $\lambda^{\alpha} \notin\left(C^{-}\right)^{\alpha}$. Then:

$-L^{\alpha}=\left[\lambda^{\alpha}, \lambda^{\alpha}\right]$

- if $\lambda \geq \mathcal{X} \lambda^{\alpha}$ then there is $\delta$ such that $\lambda=\lambda^{\delta}$, and moreover, $\lambda^{\delta} \notin\left(C^{-}\right)^{\delta}$,

- if $\lambda^{\delta} \geq \mathcal{X} \lambda^{\alpha}$ and $\lambda^{\delta} \in C^{\delta}$ then $\lambda^{\alpha} \in C^{\alpha}$, and

- the map $\xi \mapsto \lambda^{\xi}$ restricts to an isomorphism between $\left(<^{\mathcal{X} / \mathcal{T}}\right)^{(\alpha)}$ and $\left(<^{\mathcal{X}}\right)^{\left(\lambda^{\alpha}\right)}$.

Proof. This is straightforward; the last clause is by induction on $\operatorname{lh}(\mathcal{X} / \mathcal{T})$.

8.7 Lemma. Let $\mathcal{X}$ be a good inflation of $\mathcal{T}$. Adopt notation as above. Then

1. $<^{\mathcal{X} / \mathcal{T}}$ is an iteration tree order on $\operatorname{lh}(\mathcal{X} / \mathcal{T})$.

2. For all $\mu<^{\mathcal{X}} \lambda<\operatorname{lh}(\mathcal{X})$, we have $\eta_{\mu} \leq^{\mathcal{X} / \mathcal{T}} \eta_{\lambda}$.

\footnotetext{
${ }^{50}$ In fact, by 8.7 below, $b_{\alpha}=[0, \eta]_{\mathcal{X}} / \mathcal{T}$.
} 
Moreover, let $\alpha \leq^{\mathcal{X} / \mathcal{T}} \beta<\operatorname{lh}(\mathcal{X} / \mathcal{T})$ with $\lambda^{\beta} \in C^{\beta}$ (so $\lambda^{\alpha} \in C^{\alpha}$ by 8.6). Then:

3. $\gamma_{\theta \kappa}^{\alpha} \in\left[\lambda^{\alpha}, \operatorname{lh}\left(\mathcal{X}^{\alpha}\right)\right) \cup \bigcup_{\delta<\mathcal{X} / \mathcal{T} \alpha} L^{\delta}$ for all $(\theta, \kappa)$.

4. Suppose $\alpha<\beta$. Let $\xi+1=\operatorname{succ}^{\mathcal{X} / \mathcal{T}}(\alpha, \beta)$ and $\gamma=\operatorname{pred}^{\mathcal{X}}\left(\lambda^{\xi+1}\right)$. Then:

(a) $\gamma \in L^{\alpha}$ and $\theta^{\alpha} \leq \theta={ }_{\operatorname{def}} f^{\alpha}(\gamma) \leq \theta^{\beta}$.

(b) For each $\theta^{\prime}<\theta$ and $\kappa$ we have $I_{\theta^{\prime}}^{\alpha}=I_{\theta^{\prime}}^{\beta} \subseteq \gamma$ and $\gamma_{\theta^{\prime} \kappa}^{\alpha}=\gamma_{\theta^{\prime} \kappa}^{\beta}<\gamma$,

(c) $I_{\theta}^{\alpha} \subsetneq I_{\theta}^{\beta}$. In fact, $\gamma_{\theta}^{\alpha}=\gamma_{\theta}^{\beta}$ but $\delta_{\theta}^{\alpha}=\gamma<^{\mathcal{X}} \delta_{\theta}^{\beta}$.

(d) If $\theta+1<\operatorname{lh}(\mathcal{T})$ then for each $\kappa<\iota\left(\operatorname{ex}_{\theta}^{\mathcal{T}}\right)$, either:

$$
\begin{aligned}
& -\pi_{\theta \kappa}^{\alpha}(\kappa)<\operatorname{cr}\left(E_{\zeta^{\xi}}^{\mathcal{X}}\right) \text { and } \gamma_{\theta \kappa}^{\alpha}=\gamma_{\theta \kappa}^{\beta}, \\
& -\pi_{\theta \kappa}^{\alpha}(\kappa) \geq \operatorname{cr}\left(E_{\zeta^{\xi}}^{\mathcal{X}}\right) \text { and } \gamma_{\theta \kappa}^{\alpha}=\gamma<^{\mathcal{X}} \gamma_{\theta \kappa}^{\beta} .
\end{aligned}
$$

Proof. By induction on $\operatorname{lh}(\mathcal{X})$.

Part 1: By induction, we may assume that $\operatorname{lh}(\mathcal{X})=\lambda^{\alpha}+1$. By 8.4 and induction, it suffices to verify that $b_{\alpha}=[0, \alpha)_{\mathcal{X} / \mathcal{T}}$ is linearly ordered by $<\mathcal{X} / \mathcal{T}$ and closed below $\alpha$. Let $\delta, \varepsilon \in b_{\alpha}$. We can fix $\delta^{\prime}, \varepsilon^{\prime}<^{\mathcal{X}} \lambda^{\alpha}$ with $\delta \leq{ }^{\mathcal{X} / \mathcal{T}} \eta_{\delta^{\prime}}$ and $\varepsilon \leq^{\mathcal{X} / \mathcal{T}} \eta_{\varepsilon^{\prime}}$. We may assume that $\delta^{\prime} \leq{ }^{\mathcal{X}} \varepsilon^{\prime}$. Note $\eta_{\delta^{\prime}} \leq \eta_{\varepsilon^{\prime}}<\alpha$. By induction with part 2 then, $\eta_{\delta^{\prime}} \leq^{\mathcal{X} / \mathcal{T}} \eta_{\varepsilon^{\prime}}$. So if $\bar{\delta}=\eta_{\varepsilon^{\prime}}$ or $\varepsilon=\eta_{\varepsilon^{\prime}}$ then by transitivity, we are done, and otherwise, use the inductive hypothesis that $\left[0, \eta_{\varepsilon^{\prime}}\right)_{\mathcal{X} / \mathcal{T}}$ is linearly ordered by $<^{\mathcal{X} / \mathcal{T}}$. Finally, $b_{\alpha}$ is closed below $\alpha$, by induction and because if $\alpha$ is a limit then $b_{\alpha}$ is unbounded in $\alpha$ and linearly ordered by $<^{\mathcal{X} / \mathcal{T}}$.

For parts 2-4, we consider a few cases:

Case $1 \cdot \ln (\mathcal{X} / \mathcal{T})=\iota=1$.

This case is trivial.

CASE 2. $\iota=\xi+2$.

We may assume that $\mathcal{X}=\mathcal{X}_{\xi+1}$. Let $\alpha=\operatorname{pred}^{\mathcal{X} / \mathcal{T}}(\xi+1)$ and $\gamma=$ $\operatorname{pred}^{\mathcal{X}}\left(\lambda^{\xi+1}\right)$, so $\gamma \in L^{\alpha}$. Let $\theta=f^{\alpha}(\gamma)$. Then $\gamma=\delta_{\theta}^{\alpha}$ and $\Pi^{\xi+1} \uparrow(\theta+1)$ is the $E_{\xi}^{\mathcal{X}}$-inflation of $\Pi^{\alpha} \uparrow(\theta+1)$, and then $\mathcal{X}_{\xi+1}$ is given by then copying $\mathcal{T} \uparrow\left[\theta, \operatorname{lh}(\mathcal{T})\right.$ ), starting from $\Pi^{\xi+1} \uparrow(\theta+1)$ (via the one-step extension at successor stages, and copying at limits).

Consider part 2. If $\lambda=\lambda^{\xi+1}$ then the property holds for $\lambda$ (and corresponding $\mu<^{\mathcal{X}} \lambda$ ) directly by definition of $<^{\mathcal{X} / \mathcal{T}}$. We now proceed by a sub-induction through $\lambda>\lambda^{\xi+1}$. By the sub-induction, we may assume that $\lambda=\varepsilon+1$ and $\operatorname{pred}^{\mathcal{X}}(\varepsilon+1)=v \notin L^{\xi+1}$. Now $E_{\varepsilon}^{\mathcal{X}}$ is copied from $\mathcal{T}$. Let $\theta^{\prime}$ be such that $\theta^{\prime}-f^{\xi+1}(\theta)=\varepsilon-\lambda^{\xi+1}$, so $E_{\varepsilon}^{\mathcal{X}}$ is the copy of $E_{\theta^{\prime}}^{\mathcal{T}}$. Let $\kappa=\operatorname{cr}\left(E_{\theta^{\prime}}^{\mathcal{T}}\right)$. Then $v=\gamma_{\theta^{\prime} \kappa}^{\alpha}$, and by induction with parts 3 and 4 , therefore $\eta_{v}<^{\mathcal{X} / \mathcal{T}} \alpha=\eta_{\lambda^{\alpha}}$. But now if $\mu<^{\mathcal{X}} \lambda$ then $\mu \leq^{\mathcal{X}} v$, so by induction, $\eta_{\mu} \leq^{\mathcal{X} / \mathcal{T}} \eta_{v}$, so $\eta_{\mu}<^{\mathcal{X} / \mathcal{T}} \eta_{\lambda}=\xi+1$

Parts 3 and 4 are straightforward consequences of how $\Pi^{\xi+1}$ is produced from $\Pi^{\alpha}$.

CASE 3. $\iota=\alpha+1$ where $\alpha$ is a limit, and $\lambda^{\alpha}$ is a $(\mathcal{T}, \mathcal{X})$-limit.

Consider part 3. If $\theta>f^{\alpha}\left(\lambda^{\alpha}\right)=\theta^{\alpha}$ then $\gamma_{\theta \kappa}^{\alpha}>\lambda^{\alpha}$, so $\gamma_{\theta \kappa}^{\alpha} \in\left[\lambda^{\alpha}, \operatorname{lh}\left(\mathcal{X}^{\alpha}\right)\right)$. And if $\theta=\theta^{\alpha}$ then by the case hypothesis, $\lambda^{\alpha}=\gamma_{\theta}^{\alpha}=\delta_{\theta}^{\alpha}$, and $\gamma_{\theta \kappa}^{\alpha}=\lambda^{\alpha}$ for 
each $\kappa$. So suppose $\theta<\theta^{\alpha}$ and fix $\kappa$. Then $\gamma_{\theta \kappa}^{\alpha}=\gamma_{\mu ; \theta \kappa}$ for all sufficiently large $\mu<^{\mathcal{X}} \lambda^{\alpha}$. Fix such $\mu ;{ }^{51}$ we may choose $\mu$ with $f(\mu)>\theta$ where $f=f^{\mathcal{T} \rightsquigarrow \mathcal{X}}$. We have $\gamma_{\mu ; \theta \kappa}=\gamma_{\theta \kappa}^{\eta_{\mu}}$. But then

$$
\gamma_{\theta \kappa}^{\alpha}=\gamma_{\mu ; \theta \kappa}=\gamma_{\theta \kappa}^{\eta_{\mu}} \in\left[0, \eta_{\mu}\right]_{\mathcal{X} / \mathcal{T}} \subseteq[0, \alpha)_{\mathcal{X} / \mathcal{T}}
$$

by induction and definition, which suffices.

Part 2 is proved much like in the successor case, combined with considerations as above. Part 4 is easy (note the " $\alpha$ " there is not the $\alpha$ of the case hypothesis).

CASE $4 . \alpha$ is a limit but $\lambda^{\alpha}$ is not a $(\mathcal{T}, \mathcal{X})$-limit.

Part 3: For $\theta \neq \theta^{\alpha}=f^{\alpha}\left(\lambda^{\alpha}\right)$, it is basically as before. Consider $\theta=\theta^{\alpha}$. Fix $\kappa<\operatorname{OR}\left(M_{\theta^{\alpha}}^{\mathcal{T}}\right)$, with $\kappa<\iota_{\alpha}^{\mathcal{T}}$ if $\theta^{\alpha} \in \operatorname{lh}(\mathcal{T})^{-}$. Choose $\mu<^{\mathcal{X}} \lambda^{\alpha}$ large enough that $\left(\mu, \lambda^{\alpha}\right) \mathcal{X}$ does not drop, $f(\mu)=\theta^{\alpha}$ and

$$
\text { if } \pi_{\theta^{\alpha} \kappa}^{\alpha}(\kappa)<\delta\left(\mathcal{X}\left\lceil\lambda^{\alpha}\right) \text { then } \pi_{\theta^{\alpha} \kappa}^{\alpha}(\kappa)<\operatorname{cr}\left(i_{\mu \lambda^{\alpha}}^{\mathcal{X}}\right) .\right.
$$

Then if $\gamma_{\theta^{\alpha} \kappa}^{\alpha}<\lambda^{\alpha}$ then $\gamma_{\theta^{\alpha} \kappa}^{\alpha}=\gamma_{\mu ; \theta^{\alpha} \kappa}$, so the property for $\left(\theta^{\alpha}, \kappa\right)$ follows by induction as before.

Part 2 again follows by combining such considerations with the argument from the successor case. Part 4 is again easy.

\subsection{Tree embeddings of the factor tree}

8.8 Definition. Let $\mathcal{X}$ be a good inflation of $\mathcal{T}$. Adopt notation from Lemma 8.7(4). Then $\lambda^{\alpha \beta}$ denotes $\gamma, \theta^{\alpha \beta}$ denotes $f^{\alpha}\left(\lambda^{\alpha \beta}\right)$, and $\kappa^{\alpha \beta}$ denotes the least $\kappa$ such that $\pi_{\theta \kappa}^{\alpha}(\kappa) \geq \operatorname{cr}\left(E_{\zeta^{\xi}}^{\mathcal{X}}\right)$ where $\theta=\theta^{\alpha \beta}$ (because $\lambda^{\beta} \in C^{\beta}$, this makes sense and holds of $\kappa=\operatorname{ind}\left(E_{\theta}^{\mathcal{T}}\right)$ if $\theta+1<\operatorname{lh}(\mathcal{T})$, and holds of $\kappa=\operatorname{OR}\left(M_{\theta}^{\mathcal{T}}\right)$ otherwise).

8.9 Definition. Let $\mathcal{X}$ be a good inflation of $\mathcal{T}$. Adopt notation as before. Let $\alpha \leq^{\mathcal{X} / \mathcal{T}} \beta<\operatorname{lh}(\mathcal{X} / \mathcal{T})$ with $\lambda^{\beta} \in C^{\beta}$. We define a (putative, verified in 8.11) tree embedding $\Pi^{\alpha \beta}: \mathcal{X}^{\alpha} \hookrightarrow \mathcal{X}^{\beta}$ as follows. Write $\gamma_{\lambda}^{\alpha \beta}=\gamma_{\Pi^{\alpha \beta} \lambda}$ etc. It suffices to specify $I_{\lambda}^{\alpha \beta}=\left[\gamma_{\lambda}^{\alpha \beta}, \delta_{\lambda}^{\alpha \beta}\right]$ for each $\lambda<\operatorname{lh}\left(\mathcal{X}^{\alpha}\right)$. We set:

$$
\begin{aligned}
& -I_{\lambda}^{\alpha \beta}=[\lambda, \lambda] \text { if } \alpha=\beta \text { or } \lambda<\lambda^{\alpha} . \\
& -I_{\lambda^{\alpha}}^{\alpha \beta}=\left[\lambda^{\alpha}, \delta_{\theta^{\alpha}}^{\beta}\right]_{\mathcal{X}^{\beta}} \text { if } \alpha<\beta . \\
& -I_{\lambda}^{\alpha \beta}=I_{f^{\alpha}(\lambda)}^{\beta} \text { if } \alpha<\beta \text { and } \lambda>\lambda^{\alpha} .
\end{aligned}
$$

8.10 Lemma. Let $\mathcal{T}, \mathcal{X}, \alpha, \beta$ be as in 8.9, and $\lambda \geq \lambda^{\alpha}$. Let

$-\varepsilon$ be the supremum of $\alpha$ and all $\xi+1 \leq^{\mathcal{X} / \mathcal{T}} \beta$ such that $\theta^{\xi+1}<f^{\alpha}(\lambda)$,

- $\varepsilon^{\prime}$ be the supremum of all $\xi \in[\alpha, \beta]_{\mathcal{X} / \mathcal{T}}$ such that $\theta^{\xi} \leq f^{\alpha}(\lambda)$.

\footnotetext{
${ }^{51}$ In an earlier draft of this paper, $\mu$ was supposedly chosen independent of $\kappa$. But this need not actually be possible.
} 
Then:

$-\left\langle\theta_{\xi}\right\rangle_{\xi \in[\alpha, \beta]_{\mathcal{X} / \mathcal{T}}}$ is continuous, monotone increasing, so $\theta^{\varepsilon} \leq \theta^{\varepsilon^{\prime}} \leq f^{\alpha}(\lambda)$.

$$
\begin{aligned}
& -\gamma_{\lambda}^{\alpha \beta}=\lambda^{\varepsilon}+\left(f^{\alpha}(\lambda)-\theta^{\varepsilon}\right), \text { so } f^{\varepsilon}\left(\gamma_{\lambda}^{\alpha \beta}\right)=f^{\alpha}(\lambda)=f^{\beta}\left(\gamma_{\lambda}^{\alpha \beta}\right) . \\
& -\delta_{\lambda}^{\alpha \beta}=\lambda^{\varepsilon^{\prime}}+\left(f^{\alpha}(\lambda)-\theta^{\varepsilon^{\prime}}\right), \text { so } f^{\varepsilon^{\prime}}\left(\delta_{\lambda}^{\alpha \beta}\right)=f^{\alpha}(\lambda)=f^{\beta}\left(\delta_{\lambda}^{\alpha \beta}\right) .
\end{aligned}
$$

Proof. By induction on $\beta$. When $\beta=\alpha$ it is trivial, and for successor $\beta$ it follows directly from the definitions. So suppose $\beta$ is a limit. If $\theta^{\xi}=\theta^{\beta}$ for some $\xi<\mathcal{X} / \mathcal{T} \beta$ then it is just like in the successor case, so suppose otherwise, that is, $\lambda^{\beta}$ is a $(\mathcal{T}, \mathcal{X})$-limit. Then note that

$$
\theta^{\beta}=f^{\beta}\left(\lambda^{\beta}\right)=\sup _{\lambda<{ }^{\mathcal{X}} \lambda^{\beta}} f^{\mathcal{T} \rightsquigarrow \mathcal{X}}(\lambda)=\sup _{\eta<\mathcal{X} / \mathcal{T} \beta} \theta^{\eta}
$$

and for $\theta<\theta^{\beta}$,

$$
I_{\theta}^{\beta}=\lim _{\lambda<\mathcal{X}^{\beta}} I_{\lambda ; \theta}^{\mathcal{T} \rightsquigarrow \mathcal{X}}=\lim _{\eta<\mathcal{X} / \mathcal{T} \beta} I_{\theta}^{\eta} .
$$

So for $\lambda \in\left[\lambda^{\alpha}, \lambda^{\alpha}+\left(\theta^{\beta}-\theta^{\alpha}\right)\right)$ the result follows by induction, and for larger $\lambda$ it is easy.

During the course of the proof of the following lemma we will specify notation for various embeddings which will also be needed later.

8.11 Lemma. Let $\mathcal{T}, \mathcal{X}, \alpha, \beta$ be as in 8.9. Then $\Pi^{\alpha \beta}: \mathcal{X}^{\alpha} \hookrightarrow \mathcal{X}^{\beta}$ is a bounding tree embedding.

Proof. Write $\Pi=\Pi^{\alpha \beta}$. We will show that

$$
\Pi \uparrow\left(\delta_{\theta}^{\alpha}+1\right):\left(\mathcal{X}^{\alpha}, \delta_{\theta}^{\alpha}+1\right) \hookrightarrow \mathcal{X}^{\beta}
$$

is a bounding tree embedding, by induction on $\theta<\operatorname{lh}(\mathcal{T})$. We simultaneously define embeddings $\pi_{\theta}^{\alpha \beta}, \omega_{\theta}^{\alpha \beta}$ and $\pi_{\theta \kappa}^{\alpha \beta}$ for $\kappa \leq \operatorname{OR}\left(M_{\theta}^{\mathcal{T}}\right)$, as follows, and verify that

1. $\gamma_{\gamma_{\theta}^{\alpha}}^{\Pi}=\gamma_{\theta}^{\beta}$ (and $P_{\theta}^{\alpha}=M_{\gamma_{\theta}^{\alpha}}^{\mathcal{X}^{\alpha}}$ and $P_{\theta}^{\beta}=M_{\gamma_{\theta}^{\beta}}^{\mathcal{X}^{\beta}}=P_{\gamma_{\theta}^{\alpha}}^{\Pi}$ ) and defining $\pi_{\theta}^{\alpha \beta}$ by

$$
\pi_{\theta}^{\alpha \beta}=\pi_{\gamma_{\theta}^{\alpha}}^{\Pi}: P_{\theta}^{\alpha} \rightarrow P_{\theta}^{\beta},
$$

we have $\pi_{\theta}^{\alpha \beta} \circ \pi_{\theta}^{\alpha}=\pi_{\theta}^{\beta}$.

2. $\delta_{\delta_{\theta}^{\alpha}}^{\Pi}=\delta_{\theta}^{\beta}$ (and $Q_{\theta}^{\alpha}=\operatorname{ex}_{\delta_{\theta}^{\alpha}}^{\mathcal{X}^{\alpha}}$ and $Q_{\theta}^{\beta}=\operatorname{ex}_{\delta_{\theta}^{\beta}}^{\mathcal{X}^{\beta}}=Q_{\delta_{\theta}^{\alpha}}^{\Pi}$ ), and defining $\omega_{\theta}^{\alpha \beta}$ by

$$
\omega_{\theta}^{\alpha \beta}=\omega_{\delta_{\theta}^{\alpha}}^{\Pi}: Q_{\theta}^{\alpha} \rightarrow Q_{\theta}^{\beta},
$$

we have $\omega_{\theta}^{\beta}=\omega_{\theta}^{\alpha \beta} \circ \omega_{\theta}^{\alpha}$. 
3. for each $\kappa$, letting $\psi_{\theta}(\kappa)=\pi_{\theta \kappa}^{\alpha}(\kappa)$, we have

$$
\gamma_{\gamma_{\theta \kappa}^{\alpha} \psi_{\theta}(\kappa)}^{\Pi}=\gamma_{\theta \kappa}^{\beta} \text { and } P_{\theta \kappa}^{\alpha}=M_{\gamma_{\theta \kappa}^{\alpha} \psi_{\theta}(\kappa)}^{\mathcal{X}^{\alpha}} \text { and } P_{\theta \kappa}^{\beta}=P_{\gamma_{\theta \kappa}^{\alpha} \psi_{\theta}(\kappa)}^{\Pi},
$$

and defining $\pi_{\theta \kappa}^{\alpha \beta}$ by

$$
\pi_{\theta \kappa}^{\alpha \beta}=\pi_{\gamma_{\theta \kappa}^{\alpha} \psi_{\theta}(\kappa)}^{\Pi}: P_{\theta \kappa}^{\alpha} \rightarrow P_{\theta \kappa}^{\beta},
$$

we have $\pi_{\theta \kappa}^{\beta}=\pi_{\theta \kappa}^{\alpha \beta} \circ \pi_{\theta \kappa}^{\alpha}$.

Let $\eta+1=\operatorname{succ}^{\mathcal{X} / \mathcal{T}}(\alpha, \beta)$ and let $\mu=\operatorname{pred}^{\mathcal{X}}\left(E_{\zeta^{\eta}}^{\mathcal{X}}\right)$, so $f^{\alpha}(\mu)=\theta^{\eta+1}$. For $\theta<\theta^{\eta+1}$, everything is trivial, as $\mathcal{X}^{\alpha} \uparrow(\mu+1)=\mathcal{X}^{\beta} \uparrow(\mu+1)$ and $\Pi \uparrow \mu=\mathrm{id}$ and $I_{\theta}^{\alpha}=I_{\theta}^{\beta}$ and for each $\kappa$, we have $\gamma_{\theta \kappa}^{\alpha}=\gamma_{\theta \kappa}^{\beta}$, so $P_{\theta}^{\alpha}=M_{\gamma_{\theta}^{\alpha}}^{\mathcal{X}}=P_{\theta}^{\beta}, \pi_{\theta}^{\alpha}=\pi_{\theta}^{\beta}$, $Q_{\theta}^{\alpha}=\operatorname{ex}^{M_{\delta_{\theta}^{\alpha}}^{\mathcal{X}}}=Q_{\theta}^{\beta}$, etc, and $\pi_{\theta}^{\alpha \beta}, \omega_{\theta}^{\alpha \beta}$ and $\pi_{\theta \kappa}^{\alpha \beta}$ are just the identity maps.

Now consider $\theta=\theta^{\eta+1}$. We have $\gamma_{\theta}^{\alpha} \leq^{\mathcal{X}} \mu=\delta_{\theta}^{\alpha}$. By definition of $\Pi$, if $\mu=\lambda^{\alpha}$ then

$$
\gamma_{\gamma_{\theta}^{\alpha}}^{\Pi}=\gamma_{\theta}^{\beta}=\gamma_{\theta}^{\alpha} \leq^{\mathcal{X}} \lambda^{\alpha} \text { and } \delta_{\theta}^{\alpha}=\lambda^{\alpha}<^{\mathcal{X}} \delta_{\gamma_{\theta}^{\alpha}}^{\Pi}=\delta_{\theta}^{\beta},
$$

and if $\mu>\lambda^{\alpha}$ then

$$
\gamma_{\theta}^{\alpha}=\delta_{\theta}^{\alpha}=\gamma_{\theta}^{\beta}=\mu \text { and } I_{\mu}^{\Pi}=I_{\theta}^{\beta}=\left[\mu, \delta_{\theta}^{\beta}\right]_{\mathcal{X}} .
$$

So in either case, $\gamma_{\theta}^{\alpha}=\gamma_{\theta}^{\beta}=\gamma_{\gamma_{\theta}^{\alpha}}^{\Pi}$, and $\pi_{\theta}^{\alpha}=\pi_{\theta}^{\beta}$ and $\pi_{\theta}^{\alpha \beta}=$ id, so property 1 is trivial. Also, $E_{\mu}^{\mathcal{X}^{\alpha}}=E_{\alpha}^{\mathcal{T} \rightsquigarrow \mathcal{X}_{\alpha}}$, which is the iteration image of $\pi_{\theta}^{\alpha}\left(E_{\theta}^{\mathcal{T}}\right)$, and since $I_{\theta}^{\beta}$ does not drop below the image of $\pi_{\theta}^{\beta}\left(E_{\theta}^{\mathcal{T}}\right)$, therefore $I_{\mu}^{\Pi}$ does not drop below the image of $E_{\mu}^{\mathcal{X}^{\alpha}}$. Therefore, $\Pi \uparrow(\mu+1)$ is a tree embedding. Similarly, $\Pi \uparrow(\mu+1)$ is bounding. Properties 2 and 3 follow directly from this and property 1.

Now suppose we have the induction hypotheses for $\Pi \uparrow\left(\delta_{\theta}^{\alpha}+1\right)$, where $\theta \geq \mu$. We have $\gamma_{\theta+1}^{\alpha}=\delta_{\theta}^{\alpha}+1$. Using the commutativity given by this, and the fact that tree embeddings can be freely extended (in this case by copying), we get that $\pi_{\theta+1}^{\alpha \beta}=\pi_{\gamma_{\theta+1}^{\alpha}}^{\Pi}$ is well-defined, and property 1 holds. So like before, $I_{\gamma_{\theta}^{\alpha}+1}^{\Pi}=I_{\theta+1}^{\beta}$ does not drop below the image

$$
\pi_{\gamma_{\theta+1}^{\alpha}}^{\Pi}\left(E_{\gamma_{\theta+1}^{\alpha}}^{\mathcal{X}^{\alpha}}\right)=\pi_{\theta+1}^{\beta}\left(E_{\theta+1}^{\mathcal{T}}\right)
$$

(assuming that $\theta+1<\operatorname{lh}(\mathcal{T})$; otherwise there is no drop in model at all), and $\Pi \uparrow\left(\delta_{\theta+1}^{\alpha}+1\right)$ is a bounding tree embedding. Again, properties 2 and 3 follow.

For limit $\theta$, everything fits together easily by commutativity. This completes the proof.

8.12 Definition. Let $\mathcal{T}, \mathcal{X}, \alpha, \beta$ be as in 8.9. Then for $\theta<\operatorname{lh}(\mathcal{T})$ and $\kappa \leq$ $\operatorname{OR}\left(M_{\theta}^{\mathcal{T}}\right)$ we define $\pi_{\theta}^{\alpha \beta}, \omega_{\theta}^{\alpha \beta}, \pi_{\theta \kappa}^{\alpha \beta}$ as in the proof of 8.11.

8.13 Lemma. Let $\mathcal{T}, \mathcal{X}, \alpha, \beta$ be as in 8.9 and let $\gamma \in[\alpha, \beta]_{\mathcal{X} / \mathcal{T}}$. Then: 
1. $\pi_{\theta}^{\alpha \beta}=\pi_{\theta}^{\gamma \beta} \circ \pi_{\theta}^{\alpha \gamma}$ and $\omega_{\theta}^{\alpha \beta}=\omega_{\theta}^{\gamma \beta} \circ \omega_{\theta}^{\alpha \gamma}$ and $\pi_{\theta \kappa}^{\alpha \beta}=\pi_{\theta \kappa}^{\gamma \beta} \circ \pi_{\theta \kappa}^{\alpha \gamma}$.

2. If $\theta^{\beta} \leq \theta<\theta^{\prime}<\ln (\mathcal{T})$ and $\kappa^{\prime} \leq \mathrm{OR}\left(M_{\theta^{\prime}}^{\mathcal{T}}\right)$ then ${ }^{52}$

$$
\omega_{\theta}^{\alpha \beta} \subseteq \pi_{\theta^{\prime}}^{\alpha \beta}, \omega_{\theta^{\prime}}^{\alpha \beta}, \pi_{\theta^{\prime} \kappa^{\prime}}^{\alpha \beta}
$$

and if $f^{\alpha}(\lambda)=\theta=f^{\beta}\left(\lambda^{\prime}\right)$ and $\gamma=\operatorname{ind}\left(E_{\lambda}^{\mathcal{X}^{\alpha}}\right)<\mathrm{OR}\left(M_{\lambda+1}^{\mathcal{X}^{\alpha}}\right)$ then

$$
\pi_{\theta^{\prime}}^{\alpha \beta}(\gamma)=\omega_{\theta^{\prime}}^{\alpha \beta}(\gamma)=\pi_{\theta^{\prime} \kappa^{\prime}}^{\alpha \beta}(\gamma)=\operatorname{ind}\left(E_{\lambda^{\prime}}^{\mathcal{X}^{\beta}}\right)
$$

Proof. Part 1 is proved much like the commutativity in 8.11.

Part 2 holds because $\mathcal{X}^{\beta} \uparrow\left[\lambda^{\beta}, \operatorname{lh}\left(\mathcal{X}^{\beta}\right)\right)$ is the copy of $\mathcal{X}^{\alpha} \uparrow\left[\lambda, \operatorname{lh}\left(\mathcal{X}^{\alpha}\right)\right)$, where $f^{\alpha}(\lambda)=\theta^{\beta}$, under the base copy maps $\omega_{\theta^{\beta}}^{\alpha \beta}$ and $\pi_{\theta}^{\alpha \beta}, \pi_{\theta \kappa}^{\alpha \beta}$ for $\theta \leq \theta^{\beta}$.

\section{Iterability for stacks via normal realization}

In this section we will prove the main result of the paper:

9.1 Theorem. Let $\Omega>\omega$ be regular. Let $\Sigma$ be a regularly $(\Omega+1)$-total strategy for $M$ with inflation condensation, where if $M$ is a wcpm then $M$ is slightly coherent. Then $\Sigma$ extends to a strategy $\Sigma^{*}$ for stacks of length $\Omega$. More precisely, letting $m=m^{\Sigma}:^{53}$

- if $M$ is a wcpm then $M$ is $(\Omega, \Omega+1)^{*}$-iterable,

- if $\Sigma$ is a u-strategy then $M$ is $(\mathrm{u}-m, \Omega, \Omega+1)^{*}$-iterable, and

- if $\Sigma$ is an $(m, \Omega+1)$-strategy then $M$ is $(m, \Omega, \Omega+1)^{*}$-iterable,

as witnessed by some $\Sigma^{*}$ with $\Sigma \subseteq \Sigma^{*}$.

9.2 Remark. The proof will in fact give an explicit construction of a specific such strategy $\Sigma^{*}$ from $\Sigma$, and we denote this $\Sigma^{*}$ by $\Sigma^{\text {st }}$ (see Definition 9.15). If $\Sigma$ is conveniently inflationary, then for each stack $\overrightarrow{\mathcal{T}}$ via $\Sigma^{\text {st }}$ of length $<\Omega$, we will produce a tree $\mathcal{X}$ via $\Sigma$, and, roughly, lifting maps from $\overrightarrow{\mathcal{T}}$ into $\mathcal{X}$. For MS-indexed $M$ we must also translate through u-iteration strategies. We write $\mathcal{W}^{\Sigma}(\overrightarrow{\mathcal{T}})=\mathcal{X}$. This and other notation is also recorded later in Definitions 9.11 and 9.14 . In $\S 10$ we will verify some extra properties of $\Sigma^{\text {st }}$, given that $\Sigma$ satisfies some stronger properties itself.

We also prove the following variant (the relevant definitions are in $§ 1.1$ ).

9.3 Theorem. Let $\Omega>\omega$ be regular. Let $\Sigma$ be a regularly $\Omega$-total strategy for $M$ with inflation condensation, where if $M$ is a wcpm then $M$ is slightly coherent. Then $\Sigma$ extends to a strategy $\Sigma^{*}$ for stacks of length $<\omega$. More precisely, letting $m=m^{\Sigma}$ :

\footnotetext{
${ }^{52}$ Recall that in general for tree embeddings $\Pi: \mathcal{U} \hookrightarrow \mathcal{V}$ we have for example $\omega_{\xi}^{\Pi}\lceil\iota(\operatorname{ex} \mathcal{\mathcal { U }}) \subseteq$ $\pi_{\xi^{\prime}}^{\Pi}$ for $\xi<\xi^{\prime}<\operatorname{lh}(\mathcal{U})$; here we get a little more agreement.

${ }^{53}$ See 4.36 .
} 
- if $M$ is a wcpm then $M$ is $(<\omega, \Omega)^{*}$-iterable,

- if $\Sigma$ is a u-strategy then $M$ is $(\mathrm{u}-m,<\omega, \Omega)^{*}$-iterable, and

- if $\Sigma$ is an $(m, \Omega)$-strategy $M$ is $(m,<\omega, \Omega)^{*}$-iterable,

as witnessed by some $\Sigma^{*}$ with $\Sigma \subseteq \Sigma^{*}$.

Recall that by Theorem 4.47, $(n, \Omega+1)$-iteration strategies with the DJ property for premice $M$ with $\operatorname{card}(M)<\Omega$, or with weak DJ when $M$ is countable, have strong hull condensation, hence inflation condensation, so Theorem 9.1 applies in this case. In particular:

9.4 Corollary. Let $\Omega$ be regular uncountable. Let $M$ be $\omega$-sound, $(\omega, \Omega+1)$ iterable, with $\rho_{\omega}^{M}=\omega$. Then $M$ is $(\omega, \Omega, \Omega+1)^{*}$-iterable.

Proof. The unique $(\omega, \Omega+1)$-strategy for $M$ has DJ.

We will also prove a variant of Theorem 9.1, which applies to length $\omega$ (not just length $<\omega$ ) stacks of finite normal trees, assuming only normal iterability, without any condensation assumption. It is used in [14] in the proof of solidity, etc, from normal iterability. In order to state the result we need the following definition. Recall that (putative) m-maximal stack was defined in $\S 1.1$, and $\mathcal{G}_{\text {fin }}(M, m, \Omega+1)$ in Definition 1.1. We extend this naturally as follows:

9.5 Definition. For $\mathrm{u}-m$-sound $M$, we define $\mathcal{G}_{\text {fin }}(M, \mathrm{u}-m, \Omega+1)$, and for wcpms $M$, define $\mathcal{G}_{\text {fin }}(M, \Omega+1)$, analogously to $\mathcal{G}_{\text {fin }}(M, m, \Omega+1)$.

If player II has a winning strategy for $\mathcal{G}_{\text {fin }}(M, m, \Omega+1)$ where $\Omega \geq \omega$, then clearly every putative $m$-maximal stack $\overrightarrow{\mathcal{T}}$ as in Definition 1.1 (of finite length, consisting of finite length trees) is a true stack (has wellfounded models). By a proof very similar (but simpler) to that for Theorem 9.1, we also prove the following. It needs no strategy condensation hypothesis because the relevant trees have finite length.

9.6 Theorem. Let $\Omega>\omega$ be regular. Let $\Sigma$ be a regularly $(\Omega+1)$-total preinflationary ${ }^{54}$ strategy for $M$ and $m=m^{\Sigma}$, where if $M$ is a wcpm then $M$ is slightly coherent. Then player II has a winning strategy for $\mathcal{G}_{\text {fin }}(M, m, \Omega+1)$, $\mathcal{G}_{\text {fin }}(M, \mathrm{u}-m, \Omega+1)$, or $\mathcal{G}_{\text {fin }}(M, \Omega+1)$ accordingly. Moreover, let $\overrightarrow{\mathcal{T}}=\left\langle\mathcal{T}_{i}\right\rangle_{i<\omega}$ be an m-maximal, u-m-maximal, or normal, stack on $M$ respectively. Then for all sufficiently large $i<\omega, b^{\mathcal{T}_{i}}$ does not drop in model or degree, and $M_{\infty}^{\overrightarrow{\mathcal{T}}}$ is wellfounded.

9.7 Remark. In considering the proofs to come, the reader should make one observation. The definition of $\mathcal{X}=\mathcal{W}_{\Sigma}(\overrightarrow{\mathcal{T}})$ will depend on $\overrightarrow{\mathcal{T}}$ and the restriction of $\Sigma$ to the segments of $\mathcal{X}$. We are presently assuming that $\Sigma$ is total, but if $\Sigma$ were instead a partial strategy (with inflation condensation), then everything would work as long as the segments of $\mathcal{X}$ remain in the domain of $\Sigma$. We will use this observation later to deduce 9.18, which is a variant of 9.1 for partial

\footnotetext{
${ }^{54}$ Recall that pre-inflationary does not involve any actual condensation assumption!
} 
strategies. Its statement depends on the definition of $\mathcal{W}_{\Sigma}(\overrightarrow{\mathcal{T}})$, which is spelled out in the proof, and the statements are somewhat inconvenient, so we postpone them for later (the reader who wants to know what we intend to prove in this regard in advance should consult 9.18).

\subsection{Proof of Theorems 9.1, 9.3 and 9.6: The stacks strat- egy $\Sigma^{\text {st }}$}

We first observe that it suffices to construct (appropriately definable) strategies for optimal stacks:

9.8 Lemma. Let $\Omega>\omega$ be regular and $M$ be either (i),(ii) u-m-sound, or (iii) $M S$-indexed and $m$-sound. Let $\Gamma$ be a strategy for player II in the

(i) $\mathcal{G}_{\text {opt }}(M, \mathrm{u}-m, \Omega, \Omega+1)^{*}$-iteration game, or

(ii) $\mathcal{G}_{\mathrm{opt}}^{\mathrm{unrvl}}(M, \mathrm{u}-m, \Omega, \Omega+1)^{*}$-iteration game, or

(iii) $\mathcal{G}_{\mathrm{opt}}(M, m, \Omega, \Omega+1)^{*}$-iteration game,

respectively. Then there is a strategy $\widehat{\Gamma}$ for player II in the

(i) $\mathcal{G}(M, \mathrm{u}-m, \Omega, \Omega+1)^{*}$-iteration game, or

(ii) $\mathcal{G}^{\mathrm{unrvl}}(M, \mathrm{u}-m, \Omega, \Omega+1)^{*}$-iteration game, or

(iii) $\mathcal{G}(M, m, \Omega, \Omega+1)^{*}$-iteration game,

respectively. Moreover, stacks via $\widehat{\Gamma}$ lift canonically to (optimal) stacks via $\Gamma$, and if $\Omega=\omega_{1}$ and $M \in \mathrm{HC}$ and $\Gamma^{\prime} \subseteq \mathbb{R}$ and $\widehat{\Gamma}^{\prime} \subseteq \mathbb{R}$ code $\Gamma \nmid \mathrm{HC}$ and $\widehat{\Gamma}\lceil\mathrm{HC}$ in a natural manner then $\widehat{\Gamma}^{\prime}$ is $\Delta_{1}^{1}\left(\Gamma^{\prime}\right)$, uniformly in $\Gamma$.

The analogous facts also hold for deriving

(i) $\mathcal{G}(M, \mathrm{u}-m,<\omega, \Omega)^{*}$-strategies from $\mathcal{G}_{\mathrm{opt}}(M, \mathrm{u}-m,<\omega, \Omega)^{*}$-strategies, and

(ii) $\mathcal{G}^{\mathrm{unrvl}}(M, \mathrm{u}-m,<\omega, \Omega)^{*}$-strategies from $\mathcal{G}_{\mathrm{opt}}^{\mathrm{unrvl}}(M, \mathrm{u}-m,<\omega, \Omega)^{*}$-strategies, and

(iii) $\mathcal{G}(M, m,<\omega, \Omega)^{*}$-strategies from $\mathcal{G}_{\text {opt }}(M, m,<\omega, \Omega)^{*}$-strategies.

Proof Sketch. Part (i): This is just by a standard copying construction, particularly because we are dealing with u-strategies (so there are no type 3 problems); it is in particular a simplification of the construction in [17, §7]. We officially assume that $M$ is $\lambda$-indexed, so may drop the " $\mathrm{u}$ ", but the MS-indexed case is likewise. The strategy $\widehat{\Gamma}$ is defined recursively as follows. Suppose $\overrightarrow{\mathscr{S}}$ is via $\widehat{\Gamma}$, of length $\gamma<\Omega$, and $(R, r)=\left(M_{\infty}^{\vec{S}}, \operatorname{deg}^{\overrightarrow{\mathscr{S}}}(\infty)\right)$. Then we will have a corresponding optimal stack $\overrightarrow{\mathcal{U}}$ via $\Gamma$, with last model/degree $(N, n)$, and some $R^{\prime}=R_{\gamma}^{*} \unlhd N$, with $\left(R^{\prime}, r\right) \unlhd(N, n)$, and an $r$-lifting embedding $\sigma_{\gamma}: R \rightarrow R^{\prime}$. Let $\Gamma_{R, r}$ be the $(r, \Omega+1)$-strategy for $R$ given by lifting to a tree $\mathcal{U}$ via $\Gamma_{\overrightarrow{\mathcal{U}}}$ with 
$\pi_{\gamma}$. Note that $\mathcal{U}$ will be an $n$-maximal tree on $N$, as opposed to a $r$-maximal tree on $R^{\prime}$.

That is, let $\mathcal{T}$ be a $r$-maximal tree on $R$ via $\Gamma_{R, r}$, and $\mathcal{U}$ the lift, and let $R_{\alpha}=M_{\alpha}^{\mathcal{T}}, N_{\alpha}=M_{\alpha}^{\mathcal{U}}$, and if $[0, \alpha]_{\mathcal{U}}$ does not drop below the iteration image $R^{\prime \prime}$ of $R^{\prime}$, then set $R_{\alpha}^{\prime}=R^{\prime \prime}$, and otherwise set $R_{\alpha}^{\prime}=N_{\alpha}$. Let $r_{\alpha}=\operatorname{deg}^{\mathcal{T}}(\alpha)$ and $n_{\alpha}=\operatorname{deg}^{\mathcal{U}}(\alpha)$. Then we will have $\left(R_{\alpha}^{\prime}, r_{\alpha}\right) \unlhd\left(N_{\alpha}, n_{\alpha}\right)$, and a $r_{\alpha}$-lifting embedding $\pi_{\alpha}: R_{\alpha} \rightarrow R_{\alpha}^{\prime}$, where $\pi_{0}=\sigma_{\gamma}$, and the sequence of models and copy maps have typical commuting and agreement properties. If $\alpha+1<\operatorname{lh}(\mathcal{T})$ then $E_{\alpha}^{\mathcal{U}}=\pi_{\alpha}\left(E_{\alpha}^{\mathcal{T}}\right)$ (where $\pi_{\alpha}\left(F\left(R_{\alpha}\right)\right)=F\left(R_{\alpha}^{\prime}\right)$ ), and we proceed basically as usual, except that we can have $\alpha=\operatorname{pred}^{\mathcal{T}}(\beta+1)$ and $[0, \beta+1]_{\mathcal{T}} \cap \mathscr{D}_{\operatorname{deg}}^{\mathcal{T}}=\emptyset$ and $\left(R_{\alpha}^{\prime}, r\right) \triangleleft\left(M_{\beta+1}^{* \mathcal{U}}, \operatorname{deg}^{\mathcal{U}}(\beta+1)\right)$, even when $\beta+1 \in \mathscr{D}_{\operatorname{deg}}^{\mathcal{U}}$. It can also be that $[0, \alpha]_{\mathcal{T}} \cap \mathscr{D}_{\operatorname{deg}}^{\mathcal{T}} \neq \emptyset$ but $\left(R_{\alpha}^{\prime}, r_{\alpha}\right) \triangleleft\left(N_{\alpha}, n_{\alpha}\right)$.

Now suppose that at the beginning of round $\gamma$, player I plays $(S, s) \unlhd(R, r)$. Let $\Gamma_{S, s}$ be the $(s, \Omega+1)$-strategy for $S$ given by lifting trees $\mathscr{S}$ on $S$ to $r$ maximal trees $\mathcal{T}$ on $R$ via the identity map $S \rightarrow S \unlhd R$. This lifting is just just like the preceding one (except that maybe $R \neq R^{\prime}$ and $\sigma_{\gamma} \neq$ id above), and letting $S_{\alpha}=M_{\alpha}^{\mathscr{S}}$ and $R_{\alpha}=M_{\alpha}^{\mathcal{T}}$ and $S^{\prime}=S$, we get $S_{\alpha}^{\prime} \unlhd R_{\alpha}$ and copy maps $\varrho_{\alpha}: S_{\alpha} \rightarrow S_{\alpha}^{\prime}$. Then player II plays out round $\gamma$ using $\Gamma_{S, s}$, producing tree $\mathscr{S}_{\gamma}$, and composing the two lifts, we produce the $n$-maximal tree $\mathcal{U}_{\gamma}$ on $N$. If $\ln \left(\mathscr{S}_{\gamma}\right)=\alpha+1$ and we reach round $\gamma+1$, then we produce $R_{\gamma+1}^{*}$ in the natural way, and set

$$
\sigma_{\gamma+1}=\pi_{\alpha} \circ \varrho_{\alpha}: S_{\alpha} \rightarrow R_{\gamma+1}^{*} \unlhd N_{\alpha}
$$

(if $S_{\alpha}^{\prime} \triangleleft R_{\alpha}$ then $R_{\gamma+1}^{*}=\pi_{\alpha}\left(S_{\alpha}^{\prime}\right) \triangleleft R_{\alpha}^{\prime}$, and otherwise $R_{\gamma+1}^{*}=R_{\alpha}^{\prime}$ ).

If $\eta$ is a limit ordinal and we have defined $\overrightarrow{\mathscr{S}}$ of length $\eta$ and $\overrightarrow{\mathcal{U}}$ of length $\eta$ as above, then since $M_{\infty}^{\overrightarrow{\mathcal{U}}}$ is well-defined and wellfounded, it is easy to see that $M_{\infty}^{\overrightarrow{\mathcal{T}}}$ is also (including that player I eventually stopped artificially dropping) and we define $R_{\eta}^{*}$ and $\sigma_{\eta}$ via direct limit.

Part (i) and the corresponding definability clause now follow easily.

Part (ii) is almost the same as part (i). With notation as there, suppose $\mathcal{T}$ (played in round $\gamma$ ) has length $\alpha+1$ (with $\mathcal{T}$ unravelled), and $\mathcal{U}$ is its lift. We have $\left(R_{\alpha}^{\prime}, r_{\alpha}\right) \unlhd\left(N_{\alpha}, n_{\alpha}\right)$. If $R_{\alpha}^{\prime}=N_{\alpha}$ then $r_{\alpha} \neq n_{\alpha}$, and note then that $\mathcal{U}$ is unravelled. If instead $R_{\alpha}^{\prime} \triangleleft N_{\alpha}$ and $\mathcal{U}$ is not unravelled, then first replace $\mathcal{U}$ with $\operatorname{unrvl}(\mathcal{U})$ before continuing.

Part (iii): Fix $\Gamma$ as in (iii). If $M$ is type 3 then let $m^{\prime}=m+1$, and otherwise let $m^{\prime}=m$. Let $\Sigma$ be the corresponding $\mathcal{G}_{\mathrm{opt}}^{\text {unvl }}\left(M, \mathrm{u}-m^{\prime}, \Omega, \Omega+1\right)^{*}$-strategy (see Lemma 2.13). Let $\widehat{\Sigma}$ be defined as above. Now define $\widehat{\Gamma}$ as follows. Suppose we have defined $\overrightarrow{\mathcal{T}}$ of length $\gamma$ via $\widehat{\Gamma}$. Then $\left(M_{\gamma}, m_{\gamma}\right)=\left(M_{\infty}^{\overrightarrow{\mathcal{T}}}, \operatorname{deg}^{\overrightarrow{\mathcal{T}}}(\infty)\right)$ will be well-defined, and we will have a corresponding stack $\overrightarrow{\mathcal{U}}$ via $\widehat{\Sigma}$, of length $\gamma$, and letting $\left(M_{\gamma}^{\prime}, m_{\gamma}^{\prime}\right)=\left(M_{\infty}^{\overrightarrow{\mathcal{u}}}, \mathrm{u}-\operatorname{deg}^{\overrightarrow{\mathcal{U}}}(\infty)\right)$, we will have $M_{\gamma}=\left(M_{\gamma}^{\prime}\right)^{\mathrm{pm}}$, and $m_{\gamma}, m_{\gamma}^{\prime}$ are related according to the type of $M_{\gamma}$ as $m, m^{\prime}$ are. Suppose player I plays $\left(Q_{\gamma}, q_{\gamma}\right) \unlhd\left(M_{\gamma}, m_{\gamma}\right)$. If this is not an artificial drop, then also set $\left(Q_{\gamma}^{\prime}, q_{\gamma}^{\prime}\right)=\left(M_{\gamma}^{\prime}, m_{\gamma}^{\prime}\right)$, and then form $\left(\mathcal{T}_{\gamma}, \mathcal{U}_{\gamma}\right)$ with $\mathcal{U}_{\gamma}$ according to $\widehat{\Sigma}_{\overrightarrow{\mathcal{U}}}$ and $\mathcal{T}_{\gamma}$ its translation. If there is an artificial drop, then let $q_{\gamma}^{\prime}=q_{\gamma}+1$ if $Q_{\gamma}$ is type 3 , and $q_{\gamma}^{\prime}=q_{\gamma}$ otherwise, $Q_{\gamma}^{\prime}=Q_{\gamma}$ (recalling that if $M_{\gamma}^{\prime} \neq M_{\gamma}$ then $m_{\gamma}=0$, 
so $Q_{\gamma} \triangleleft M_{\gamma}$ and since $M_{\gamma}=\left(M_{\gamma}^{\prime}\right)^{\mathrm{pm}}$, therefore $\left.Q_{\gamma} \triangleleft M_{\gamma}^{\prime}\right)$, and noting that $\left(Q_{\gamma}, q_{\gamma}^{\prime}\right) \triangleleft\left(M_{\gamma}^{\prime}, m_{\gamma}^{\prime}\right)$, now play $\mathcal{T}_{\gamma}, \mathcal{U}_{\gamma}$ as before, but on $\left(Q_{\gamma}, q_{\gamma}\right)$ and $\left(Q_{\gamma}^{\prime}, q_{\gamma}^{\prime}\right)$. Note then that by Lemma 2.12, $\overrightarrow{\mathcal{T}} \wedge \mathcal{T}_{\gamma}$ and $\overrightarrow{\mathcal{U}} \wedge \mathcal{U}_{\gamma}$ again satisfy the inductive requirements.

Finally, if we have $\overrightarrow{\mathcal{T}}, \overrightarrow{\mathcal{U}}$ of limit length, then because $M_{\infty}^{\overrightarrow{\mathcal{U}}}$ is well-defined and wellfounded, and because of the correspondence of iteration maps given by Lemma $2.12, M_{\infty}^{\overrightarrow{\mathcal{T}}}$ is also well-defined and wellfounded, and the inductive hypotheses hold.

The lemma easily follows.

So by the lemma, in order to prove Theorems 9.1 and 9.3, we just need to construct appropriate strategies for optimal stacks. In the construction we work with conveniently inflationary strategies, and directly construct a convenient strategy (for optimal stacks), and then derive from this inconvenient strategies (also for optimal stacks). This derivation is is quickly dispensed with and we deal with it first. Consider the case of 9.1. Suppose $M$ is MS-indexed. We have the normal strategy $\Sigma$ for $M$. Let $\ell=m+1$ if $M$ is type 3; otherwise let $\ell=m$. Let $\Gamma$ be the $(\mathrm{u}-\ell, \Omega+1)$-strategy for $M$ corresponding to $\Sigma$ (see 2.13). By definition, $\Gamma$ has inflation condensation. Suppose that the theorems hold with respect to convenient strategies (hence for $\Gamma)$. Let $\Gamma^{*}$ be a $(\mathrm{u}-\ell, \Omega, \Omega+1)^{*}$ strategy for $M$ such that $\Gamma \subseteq \Gamma^{*}$. Let $\Sigma^{*}$ be the $(m, \Omega, \Omega+1)^{*}$-strategy for $M$ determined by $\Gamma^{*}$ (that is, by restricting $\Gamma^{*}$ to unravelled stacks, we get an unravelled strategy, and this corresponds to $\left.\Sigma^{*}\right)$. Then $\Sigma \subseteq \Sigma^{*}$, so we are done. For 9.3 it is completely analogous.

We now consider convenient strategies. We only literally give the proof for $\mathrm{u}$-strategies, as the coarse case is mainly a simplification thereof, but we will point out where we use slight coherence. So fix $\Omega$ and a u- $m$-strategy $\Sigma$ for $M$ as in 9.1 or 9.3 . We will construct an appropriate stacks strategy $\Sigma^{*}$ for $M$, extending $\Sigma$. We first give a sketch of the process. For the purposes of this sketch, we consider literally the case of 9.1 , so $\Sigma$ is an (u- $m, \Omega+1)$-strategy (but in either case, the constructions agree over their restriction to an $(\mathrm{u}-m,<\omega, \Omega)^{*}$ strategy).

For stacks $\overrightarrow{\mathcal{T}}$ on $M$ via $\Sigma^{*}$ of length $<\Omega$, we will construct a corresponding normal tree $\mathcal{Y}$ via $\Sigma$, of successor length, which "absorbs" $\overrightarrow{\mathcal{T}}$, and in particular, such that $M_{\infty}^{\overrightarrow{\mathcal{T}}}$ embeds into $M_{\infty}^{\mathcal{Y}}$ (here, $M_{\infty}^{\overrightarrow{\mathcal{T}}}$ will be well-defined as we will also verify that $\overrightarrow{\mathcal{T}}$ has only finitely many drops along its main branch, by showing that drops in model in $\overrightarrow{\mathcal{T}}$ correspond suitably to drops in model in $\mathcal{Y})$. In the case of a stack $(\mathcal{T}, \mathcal{U})$ of length 2 (with $\mathcal{T}, \mathcal{U}$ normal), $\mathcal{Y}$ will be an inflation of $\mathcal{T}$, with the $\mathcal{T}$-inflationary extenders being just copies of extenders used in $\mathcal{U}$. This easily yields a strategy for finite stacks of trees. In the limit case, for a stack $\overrightarrow{\mathcal{T}}$ of length $\eta$, we will have a sequence of inflations $\left\langle\mathcal{Y}_{\alpha}\right\rangle_{\alpha<\eta}$. We will define $\mathcal{Y}=\mathcal{Y}_{\eta}$ as the minimal simultaneous inflation of $\left\{\mathcal{Y}_{\alpha}\right\}_{\alpha<\eta}$. The commutativity lemma 6.2 is the key to seeing that everything fits together appropriately.

Here is a more detailed sketch (cf. Figure 8 on page 94 , where $O_{n}=M_{\infty}^{\overrightarrow{\mathcal{T}} n}$; the figure incorporates more detail than given in this sketch). The trees mentioned 
below are of successor length and the inflations are terminal. Given a normal tree $\mathcal{T}_{0}$ on $M$, via $\Sigma$, and a normal tree $\mathcal{T}_{1}$ on $M_{\infty}^{\mathcal{T}_{0}}$, with $\left(\mathcal{T}_{0}, \mathcal{T}_{1}\right)$ via $\Sigma^{*}$, letting $\mathcal{Y}_{1}=\mathcal{T}_{0}$, we will define an inflation $\mathcal{Y}_{2}$ of $\mathcal{Y}_{1}$, such that $M_{\infty}^{\mathcal{T}_{1}}$ embeds into $M_{\infty}^{\mathcal{Y}_{2}}$ (the reason for this misalignment of integers will become clearer later). The fact that $\Sigma$ has inflation condensation will ensure that this process does not break down. Then, given a normal tree $\mathcal{T}_{2}$ on $M_{\infty}^{\mathcal{T}_{1}}$, with $\left(\mathcal{T}_{0}, \mathcal{T}_{1}, \mathcal{T}_{2}\right)$ via $\Sigma^{*}$, we will define an inflation $\mathcal{Y}_{3}$ of $\mathcal{Y}_{2}$, such that $M_{\infty}^{\mathcal{T}_{2}}$ embeds into $M_{\infty}^{\mathcal{Y}_{3}}$. And so on for finite stacks.

Now let $\overrightarrow{\mathcal{T}}=\left\langle\mathcal{T}_{n}\right\rangle_{n<\omega}$ be a stack of normal trees via $\Sigma^{*}$. We will have a sequence $\left\langle\mathcal{Y}_{n}\right\rangle_{n<\omega}$ as above, where $\mathcal{Y}_{0}$ is the trivial tree on $M$. So $\mathcal{Y}_{l+2}$ is an inflation of $\mathcal{Y}_{l+1}$ is an inflation of $\mathcal{Y}_{l}$. Using 6.2, we will have that for $n_{0}<$ $n_{1}<n_{2}, \mathcal{Y}_{n_{2}}$ is a inflation of $\mathcal{Y}_{n_{1}}$ is an inflation of $\mathcal{Y}_{n_{0}}$, everything commutes (and all these inflations are also terminal). Let us assume for simplicity that all trees are terminally non-dropping. Then for each $n_{0}<n_{1}, \mathcal{Y}_{n_{1}}$ will be $\mathcal{Y}_{n_{0}}$-terminally-non-dropping, and the iteration embeddings

$$
i^{\mathcal{T}_{n}}: M_{\infty}^{\overrightarrow{\mathcal{T}}\lceil n} \rightarrow M_{\infty}^{\overrightarrow{\mathcal{T}}\lceil(n+1)}=M_{\infty}^{\mathcal{T}_{n}}
$$

and the final inflation copy maps

$$
\pi_{n_{0} n_{1}}={ }_{\operatorname{def}} \pi_{\infty}^{\mathcal{Y}_{n_{0}} \rightsquigarrow \mathcal{Y}_{n_{1}}}: M_{\infty}^{\mathcal{Y}_{n_{0}}} \rightarrow M_{\infty}^{\mathcal{Y}_{n_{1}}}
$$

will commute with the maps $\varsigma_{n_{0}}, \varsigma_{n_{1}}$ where

$$
\varsigma_{n}: M_{\infty}^{\overrightarrow{\mathcal{T}}[n} \rightarrow M_{\infty}^{\mathcal{Y}_{n}},
$$

is the lifting map mentioned in the previous paragraph. Therefore the direct limit $M_{\infty}^{\overrightarrow{\mathcal{T}}}$ embeds into the direct limit of the models $M_{\infty}^{\mathcal{Y}_{n}}$ under the maps $\pi_{n_{0}, n_{1}}$. We will set $\mathcal{Y}_{\omega}$ to be the minimal simultaneous inflation of $\left\{\mathcal{Y}_{n}\right\}_{n<\omega}$. Then $\mathcal{Y}_{\omega}$ will be an $\mathcal{Y}_{n}$-terminal inflation of $\mathcal{Y}_{n}$ for each $n$, and because of our extra assumptions here regarding (non-)dropping, $\mathcal{Y}_{\omega}$ will be $\mathcal{Y}_{n}$-terminally-nondropping for each $n$. Defining

$$
\pi_{n \omega}=\pi_{\infty}^{\mathcal{Y}_{n} \rightsquigarrow \mathcal{Y}_{\omega}}: M_{\infty}^{\mathcal{Y}_{n}} \rightarrow M_{\infty}^{\mathcal{Y}_{\omega}},
$$

then by 6.2 , we have

$$
\pi_{n_{0} \omega}=\pi_{n_{1} \omega} \circ \pi_{n_{0} n_{1}}
$$

for $n_{0}<n_{1}<\omega$. Therefore $M_{\infty}^{\mathcal{Y}_{\omega}}$ absorbs the direct limit of the models $M_{\infty}^{\mathcal{Y}_{n}}$, and so absorbs $M_{\infty}^{\overrightarrow{\mathcal{T}}}$, and in particular, $M_{\infty}^{\overrightarrow{\mathcal{T}}}$ is wellfounded. The process then continues through longer stacks in the same manner.

Note that our proof that the minimal simultaneous inflation exists requires that $\Sigma$ be an (u- $m, \Omega+1)$-strategy; thus, under the weaker assumption of (u- $m, \Omega$ )-iterability we do not see how to deal with limit stages, and so only obtain an $(\mathrm{u}-m,<\omega, \Omega)^{*}$-strategy. There are some further details involved in dealing with dropping trees and inflations, but these are straightforward using 6.2 .

We now proceed to the details. 


\subsubsection{Stacks of length 2}

Before we begin with the main construction, we prove a fine structural lemma. The lemma, however, is only needed in the proof of a detail which the reader might prefer to ignore at a first pass. We prove it only for $\lambda$-indexing, for notational simplicity; the analogue also holds for MS-indexing, however (see $[14, \S 6]$ for related material).

9.9 Definition. Let $k<\omega$ and $S$ be a $k$-sound $\lambda$-indexed premouse. Then $\operatorname{wcof}_{k+1}^{S}$ (for weak cofinality) denotes the least $\tau$ such that

$$
\exists q \in S\left[\operatorname{Hull}_{\mathrm{r} \Sigma_{k+1}}^{S}(\tau \cup\{q\}) \text { is cofinal in } \rho_{k}^{S}\right] .
$$

Note this is the least $\tau \leq \rho_{k+1}^{S}$ such that either $\tau=\rho_{k+1}^{S}$ or there is a $r \Sigma_{k+1^{-}}^{S}$ function $f: \tau \rightarrow \rho_{k}^{S}$ which is cofinal, strictly increasing and continuous.

9.10 Lemma. Let $R, S$ be $(k+1)$-sound $\lambda$-indexed premice and $\pi: R \rightarrow S$ a near $(k+1)$-embedding. Then either:

$$
\begin{aligned}
& -\operatorname{wcof}_{k+1}^{R}<\rho_{k+1}^{R} \text { and } \pi\left(\operatorname{wcof}_{k+1}^{R}\right)=\operatorname{wcof}_{k+1}^{S} \text {, or } \\
& -\operatorname{wcof}_{k+1}^{R}=\rho_{k+1}^{R} \text { and } \operatorname{wcof}_{k+1}^{S}=\rho_{k+1}^{S} .
\end{aligned}
$$

Proof. Recall that either $\rho_{k}^{R}=\mathrm{OR}^{R}$ and $\rho_{k}^{S}=\mathrm{OR}^{S}$, or $\pi\left(\rho_{k}^{R}\right)=\rho_{k}^{S}$. And $\pi\left(\rho_{k+1}^{R}\right) \geq \rho_{k+1}^{S}$ by $\mathrm{r} \Sigma_{k+2}$-elementarity. ${ }^{55}$ Now given $\tau<\rho_{k+1}$ and some parameter $q$, it is an $\operatorname{r\Pi }_{k+2}\left(\tau, q, \rho_{k}\right)$ assertion that

$$
\text { "Hull } k+1(\tau \cup\{q\}) \text { is cofinal in } \rho_{k} " .
$$

And given $\tau<\rho_{k+1}$, it is an $\mathrm{r} \Pi_{k+2}\left(\tau, \rho_{k}\right)$ assertion that

$$
\text { " } \forall \alpha<\tau \forall q\left[\operatorname{Hull}_{k+1}^{R}(\alpha \cup\{q\}) \text { is bounded in } \rho_{k}\right] \text { ". }
$$

(For this can be expressed as "For every $\alpha<\tau$ and $q$ and every $T \in T_{k+1}$ such that $T$ is a theory in parameters $\alpha \cup\{q\}$, there is some $T^{\prime} \in T_{k}$ which codes witnesses to all $\mathrm{r} \Sigma_{k+1}$ formulas in $T$ "; here coding a witness is in the style described in $[4, \S 2]$.$) Likewise, it is an \mathrm{r}_{k+2}\left(\rho_{k}\right)$ assertion that

$$
\text { " } \forall \alpha<\rho_{k+1} \forall q\left[\operatorname{Hull}_{k+1}^{R}(\alpha \cup\{q\}) \text { is bounded in } \rho_{k}\right] \text { ". }
$$

Since $\pi$ is a near $(k+1)$-embedding, the lemma follows.

We now begin the main proof for the case of realizing a stack of two normal trees via a single normal tree. For this case we only assume in general that $\Sigma$ is an (u- $m, \Omega)$-strategy, (not $(\mathrm{u}-m, \Omega+1)$ ). Let $\mathcal{T}$ be an $\mathrm{u}-m$-maximal tree on $M$ of successor length $<\Omega$, via $\Sigma$. Let $N=M_{\infty}^{\mathcal{T}}$ and $n=\mathrm{u}-\operatorname{deg}^{\mathcal{T}}(\infty)$.

We describe a

$$
(\mathrm{u}-n, \Omega) \text {-iteration strategy } \Upsilon_{\mathcal{T}}^{\Sigma} \text { for } N \text {. }
$$

\footnotetext{
${ }^{55}$ Here $\pi\left(\mathrm{OR}^{R}\right)$ denotes $\mathrm{OR}^{S}$.
} 


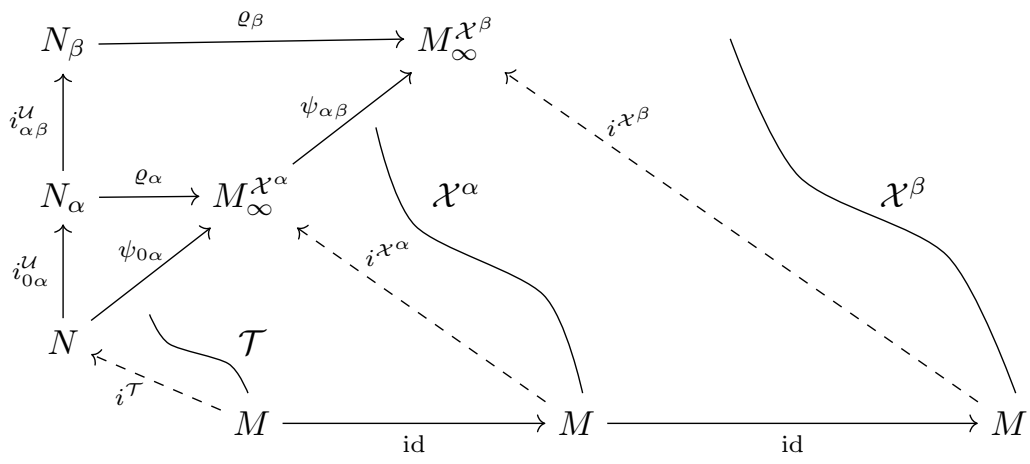

Figure 6: Commutativity for maps relating to $\Upsilon_{\mathcal{T}}^{\Sigma}$ assuming $[0, \beta]_{\mathcal{U}} \cap \mathscr{D}^{\mathcal{U}}=\emptyset$ (see conditions N7 and N8). The curved lines represent the iteration trees $\mathcal{T}$, $\mathcal{X}^{\alpha}, \mathcal{X}^{\beta}$. The solid arrows commute. The dashed arrows exist iff $b^{\mathcal{T}} \cap \mathscr{D}^{\mathcal{T}}=\emptyset$, and when they exist, they commute with the other maps.

In order to do this, we lift $\mathrm{u}-n$-maximal trees $\mathcal{U}$ on $N$ via $\Upsilon_{\mathcal{T}}^{\Sigma}$ of length $\leq \Omega$ to $\mathrm{u}-m$-maximal trees $\mathcal{X}$ on $M$ via $\Sigma$. We write $\mathcal{W}_{\mathcal{T}}^{\Sigma}(\mathcal{U})$ for $\mathcal{X}$. ${ }^{56}$ Here $\mathcal{X}$ will depend on $\Sigma, \mathcal{T}$ and the extenders used in $\mathcal{U}$, but $\mathcal{X}$ will determine the branches chosen in $\mathcal{U}$. Moreover, for limits $\eta<\operatorname{lh}(\mathcal{U})$ we will have

$$
\mathcal{X}^{\prime}={ }_{\operatorname{def}} \mathcal{W}_{\mathcal{T}}^{\Sigma}(\mathcal{U}\lceil\eta) \triangleleft \mathcal{X},
$$

with $\operatorname{lh}\left(\mathcal{X}^{\prime}\right)$ a limit, and $\Sigma\left(\mathcal{X}^{\prime}\right)$ determines $[0, \eta)_{\mathcal{U}}$. If $\Sigma$ extends to a $(\mathrm{u}-m, \Omega+1)$ strategy, then so will $\Upsilon_{\mathcal{T}}^{\Sigma}$. We will also define $\mathcal{W}_{\mathcal{T}}^{\Sigma}(\mathcal{U})$ when $\operatorname{lh}(\mathcal{U})=\Omega+1$, but this tree can have length $>\Omega+1$, and so be not literally via $\Sigma$. For now we assume that $\ln (\mathcal{U}) \leq \Omega$, and then later consider the extension to $\Omega+1$.

The tree $\mathcal{X}$ will be a non- $\mathcal{T}$-pending inflation of $\mathcal{T}$, via $\Sigma$, with associated objects

$$
\left(t, C, \ldots, \lambda^{\alpha}, \mathcal{X}^{\alpha}, \ldots\right)=\left(t, C, \ldots, \lambda^{\alpha}, \mathcal{X}^{\alpha}, \ldots\right)^{\mathcal{T} \rightsquigarrow \mathcal{X}} .
$$

The $\mathcal{T}$-inflationary extenders $E_{\zeta^{\alpha}}^{\mathcal{X}}$ used in $\mathcal{X}$ will be copies of extenders from $\mathcal{U}$ (and of course, the others are copied from $\mathcal{T}$ ). We will define a lifting map

$$
\varrho_{\alpha}: M_{\alpha}^{\mathcal{U}} \rightarrow M_{\infty}^{\mathcal{X}^{\alpha}} .
$$

We say that $\alpha$ is easy iff $\lambda^{\alpha} \notin\left(C^{-}\right)^{\alpha}$.

We will build $\mathcal{U}\left\lceil\eta,\left\langle\zeta^{\alpha}, E_{\zeta^{\alpha}}^{\mathcal{X}}\right\rangle_{\alpha+1<\eta},\left\langle\lambda^{\alpha}, \mathcal{X}^{\alpha}, \varrho_{\alpha}\right\rangle_{\alpha<\eta}\right.$, etc, thus determining

$$
\mathcal{X}\left\lceil\sup _{\alpha<\eta}\left(\lambda^{\alpha}+1\right)\right.
$$

\footnotetext{
${ }^{56}$ We use the notation $\mathcal{W}_{\mathcal{T}}^{\Sigma}(\mathcal{U})$ instead of $\mathcal{X}_{\mathcal{T}}^{\Sigma}(\mathcal{U})$ for consistency with Steel's notation, and because we will use $\mathcal{X}_{\mathcal{T}}^{\Sigma}(\mathcal{U})$ in the future for (full) normalization, as opposed to normal realization. But for consistency with the rest of the paper, we continue to use the variable $\mathcal{X}$.
} 
by induction on $\eta$, maintaining the following conditions. For $1 \leq \eta \leq \Omega$, let $\varphi(\eta)$ assert that these objects are defined and the following conditions hold $(N$ is for normal):

N1. $\mathcal{X}\left\lceil\sup _{\alpha<\eta}\left(\lambda^{\alpha}+1\right)\right.$ is via $\Sigma$ and is an inflation of $\mathcal{T}$, with the associated objects described above (in particular, for each $\alpha<\eta, \mathcal{X}^{\alpha}$ is a $\mathcal{T}$-terminal inflation of $\mathcal{T}$ and $\left.\mathcal{X} \uparrow\left(\lambda^{\alpha}+1\right)=\mathcal{X}^{\alpha} \uparrow\left(\lambda^{\alpha}+1\right)\right)$.

N2. Tree order: $\left(<^{\mathcal{U}}\right)\left\lceil\eta=\left(<^{\mathcal{X} / \mathcal{T}}\right)\lceil\eta\right.$.

N3. For $\alpha<\eta$, we have: ${ }^{57}$

$-k={ }_{\text {def }} \mathrm{u}-\operatorname{deg}^{\mathcal{U}}(\alpha) \leq \mathrm{u}-\operatorname{deg}^{\mathcal{X}^{\alpha}}(\infty)$,

- $\varrho_{\alpha}: M_{\alpha}^{\mathcal{U}} \rightarrow M_{\infty}^{\mathcal{X}^{\alpha}}$ is nice u- $k$-lifting,

$-[0, \alpha]_{\mathcal{U}} \cap \mathscr{D}^{\mathcal{U}}=\emptyset$ iff $\lambda^{\alpha} \in C^{\alpha}$.

- If $[0, \alpha]_{\mathcal{U}} \cap \mathscr{D}_{\mathrm{deg}}^{\mathcal{U}} \neq \emptyset$ then:

$-\lambda^{\alpha}+1=\ln \left(\mathcal{X}^{\alpha}\right)$,

- $\varrho_{\alpha}$ is a near u- $k$-embedding,

- if $[0, \alpha]_{\mathcal{U}} \cap \mathscr{D}^{\mathcal{U}} \neq \emptyset$ or $k+1<n$ then $k=\mathrm{u}$ - $\operatorname{deg}^{\mathcal{X}^{\alpha}}\left(\lambda^{\alpha}\right)$.

- If $\alpha$ is non-easy then $[0, \alpha]_{\mathcal{U}} \cap \mathscr{D}_{\operatorname{deg}}^{\mathcal{U}}=\emptyset$.

- If $[0, \alpha]_{\mathcal{U}} \cap \mathscr{D}_{\mathrm{deg}}^{\mathcal{U}}=\emptyset$ and $\mathcal{T}$ is terminally non-dropping then $\mathcal{X}^{\alpha}$ is terminally non-dropping and $\varrho_{\alpha}$ is a u-m-embedding.

N4. Let $\alpha<\beta<\eta$. Then:

- If $E_{\alpha}^{\mathcal{U}}=F^{M_{\alpha}^{\mathcal{U}}}$ then $\zeta^{\alpha}+1=\ln \left(\mathcal{X}^{\alpha}\right)$ and $E_{\zeta^{\alpha}}^{\mathcal{X}^{\beta}}=F^{M_{\infty}^{\mathcal{X}^{\alpha}}}$.

- If $E_{\alpha}^{\mathcal{U}} \neq F^{M_{\alpha}^{\mathcal{U}}}$ then $E_{\zeta^{\alpha}}^{\mathcal{X}^{\beta}}=\varrho_{\alpha}\left(E_{\alpha}^{\mathcal{U}}\right)$, and $\zeta^{\alpha}$ is the least $\zeta$ with $\varrho_{\alpha}\left(E_{\alpha}^{\mathcal{U}}\right) \in \mathbb{E}\left(M_{\zeta}^{\mathcal{X}^{\alpha}}\right)$.

N5. For $\alpha<\beta<\eta$, we have $\varrho_{\alpha}\left\lceil\operatorname{ind}\left(E_{\alpha}^{\mathcal{U}}\right) \subseteq \varrho_{\beta}\left(\right.\right.$ so $\left.\varrho_{\beta}\left(\widetilde{\nu}_{\alpha}^{\mathcal{U}}\right)=\widetilde{\nu}_{\zeta^{\alpha}}^{\mathcal{X}^{\beta}}\right)$, and either

$$
\begin{aligned}
& -\operatorname{ind}\left(E_{\alpha}^{\mathcal{U}}\right)<\operatorname{OR}\left(M_{\alpha+1}^{\mathcal{U}}\right) \text { and } \varrho_{\alpha+1}\left(\operatorname{ind}\left(E_{\alpha}^{\mathcal{U}}\right)\right)=\operatorname{ind}\left(E_{\zeta^{\alpha}}^{\mathcal{X}^{\beta}}\right), \text { or } \\
& -\operatorname{ind}\left(E_{\alpha}^{\mathcal{U}}\right)=\operatorname{OR}\left(M_{\alpha+1}^{\mathcal{U}}\right) \text { and } \operatorname{ind}\left(E_{\zeta^{\alpha}}^{\mathcal{X}^{\beta}}\right)=\operatorname{OR}\left(M_{\zeta^{\alpha}+1}^{\mathcal{X}^{\beta}}\right) \text { and } M_{\alpha+1}^{\mathcal{U}}, M_{\zeta^{\alpha}+1}^{\mathcal{X}^{\beta}}
\end{aligned}
$$
are active type 2 with MS-indexing.

N6. Let $\alpha \leq \beta<\eta$ be such that $\alpha$ is easy (so 8.6 applies). Then:

(a) $\gamma \mapsto \lambda^{\gamma}$ restricts to an isomorphism $\left(<^{\mathcal{U} \beta \beta+1}\right)^{(\alpha)} \rightarrow\left(<^{\mathcal{X}^{\beta}}\right)^{\left(\lambda^{\alpha}\right)}$ preserving drop structure, and above drops in model, degree structure.

\footnotetext{
${ }^{57}$ Remark 9.13 shows that this condition cannot in general be improved much.
} 
(b) Let $\alpha \leq^{\mathcal{U}} \gamma \leq \beta$, so $\gamma$ is easy, so $\operatorname{lh}\left(\mathcal{X}^{\gamma}\right)=\lambda^{\gamma}+1$ and

$$
\varrho_{\gamma}: M_{\gamma}^{\mathcal{U}} \rightarrow M_{\lambda^{\gamma}}^{\mathcal{X}^{\gamma}}
$$

Let $\gamma \leq{ }^{\mathcal{U}} \xi \leq \beta$ with $(\gamma, \xi]_{\mathcal{U}} \cap \mathscr{D}^{\mathcal{U}}=\emptyset$. Let $\psi_{\gamma \xi}=i_{\lambda^{\gamma} \lambda \xi}^{\mathcal{X}^{\xi}}$. Then

$$
\varrho_{\xi} \circ i_{\gamma \xi}^{\mathcal{U}}=\psi_{\gamma \xi} \circ \varrho_{\gamma},
$$

and if $\gamma$ is a successor then letting $\delta=\operatorname{pred}^{\mathcal{U}}(\gamma),{ }^{58}$

$$
\begin{gathered}
\gamma \in \mathscr{D}^{\mathcal{U}} \Longleftrightarrow \lambda^{\gamma} \in \mathscr{D}^{\mathcal{X}^{\gamma}}, \\
\gamma \in \mathscr{D}^{\mathcal{U}} \Longrightarrow \varrho_{\delta}\left(N_{\gamma}^{*}\right)=M_{\lambda^{\gamma}}^{* \mathcal{X}^{\gamma}}, \\
\varrho_{\gamma} \circ i_{\gamma}^{* \mathcal{U}}=i_{\lambda \gamma}^{* \mathcal{X}^{\gamma}} \circ \varrho_{\delta}\left\lceil N_{\gamma}^{*} .\right.
\end{gathered}
$$

N7. (Cf. Figure 6) Let $\alpha \leq \mathcal{U} \beta<\eta$ be such that $\beta$ is non-easy (so $[0, \beta]_{\mathcal{U}} \cap$ $\mathscr{D}_{\text {deg }}^{\mathcal{U}}=\emptyset$ and $\alpha$ is non-easy and $\mathcal{X}^{\alpha}, \mathcal{X}^{\beta}$ are $\mathcal{T}$-terminally-non-dropping). Let

$$
\psi_{\alpha \beta}=\pi_{\operatorname{lh}(\mathcal{T})-1}^{\alpha \beta}=\omega_{\operatorname{lh}(\mathcal{T})-1}^{\alpha \beta}: M_{\infty}^{\mathcal{X}^{\alpha}} \rightarrow M_{\infty}^{\mathcal{X}^{\beta}}
$$

(where $\pi_{\mathrm{lh}(\mathcal{T})-1}^{\alpha \beta}=\omega_{\mathrm{lh}(\mathcal{T})-1}^{\alpha \beta}$ are defined in 8.12 and are equal because $\theta^{\beta}+1<\operatorname{lh}(\mathcal{T})$ because $\beta$ is non-easy). Then $\psi_{\alpha \beta} \circ \varrho_{\alpha}=\varrho_{\beta} \circ i_{\alpha \beta}^{\mathcal{U}}$.

N8. (Cf. Figure 6) Let $\alpha \leq \mathcal{U} \beta<\eta$ be such that $\beta$ is easy but $\lambda^{\beta} \in C^{\beta}$. Let

$$
\psi_{\alpha \beta}=\omega_{\operatorname{lh}(\mathcal{T})-1}^{\alpha \beta}: M_{\infty}^{\mathcal{X}^{\alpha}} \rightarrow M_{\infty}^{\mathcal{X}^{\beta}} .
$$

Then $\psi_{\alpha \beta} \circ \varrho_{\alpha}=\varrho_{\beta} \circ i_{\alpha \beta}^{\mathcal{U}}$.

This completes the inductive hypotheses. Note that N7 and N8 actually have the same conclusion. We now begin the construction.

With $\mathcal{U} \uparrow 1=$ the trivial tree, $\mathcal{X}^{0}=\mathcal{T}$ and $\varrho_{0}=$ id $: N \rightarrow N, \varphi(1)$ is trivial.

Now suppose we are given $\mathcal{U}\lceil\eta$ and the other related objects, and $\varphi(\eta)$ holds; we define $\mathcal{U}\lceil\eta+1$, etc, and verify $\varphi(\eta+1)$. Suppose first that $\eta=\alpha+1$. So we have defined $\mathcal{X}^{\beta}, \varrho_{\beta}$, etc, for all $\beta \leq \alpha$ and $\zeta^{\beta}$ for all $\beta<\alpha$, and $\varphi(\alpha+1)$ holds. Let $E=E_{\alpha}^{\mathcal{U}}$.

Now $\zeta^{\alpha}$ is determined by property N4; let us observe that $\zeta^{\alpha} \geq \lambda^{\alpha}$. If $\alpha$ is a limit or $E=F^{M_{\alpha}^{\mathcal{u}}}$ this is easy; suppose $\alpha=\gamma+1$ and $E \neq F^{M_{\alpha}^{\mathcal{u}}}$. Then $\operatorname{ind}\left(E_{\gamma}^{\mathcal{U}}\right)<\operatorname{ind}(E)$, so by N5,

$$
\varrho_{\alpha}(\operatorname{ind}(E))>\varrho_{\alpha}\left(\operatorname{ind}\left(E_{\gamma}^{\mathcal{U}}\right)\right)=\operatorname{ind}\left(E_{\zeta^{\gamma}}^{\mathcal{X}^{\alpha}}\right),
$$

so $\zeta^{\alpha} \geq \zeta^{\gamma}+1=\lambda^{\alpha}$.

Now $\mathcal{X}^{\alpha+1}$ is determined by setting $F=E_{\zeta^{\alpha}}^{\mathcal{X}^{\alpha+1}}$ according to N4. By coherence, $F$ is indeed $\mathcal{X}^{\alpha} \uparrow\left(\zeta^{\alpha}+1\right)$-normal, so we can do this. (For the wcpm case, it

${ }^{58}$ The fact that if $\lambda^{\gamma} \in \mathscr{D}^{\mathcal{X}^{\gamma}}$ then $\gamma \in \mathscr{D}^{\mathcal{U}}$ depends on the fact that $\alpha$ is easy. 
is here that we use that $M$ is slightly coherent. That is, by slight coherence and 3.7, $\zeta \alpha$ is the least $\zeta$ such that either $\ln \left(\mathcal{X}^{\alpha}\right)=\zeta+1$ or $\varrho^{M_{\zeta}^{\mathcal{X}^{\alpha}}}\left(E_{\zeta}^{\mathcal{X}^{\alpha}}\right) \geq \varrho^{M_{\zeta}^{\mathcal{X}^{\alpha}}}(F)$, so $F$ is $\mathcal{X}^{\alpha} \uparrow\left(\zeta^{\alpha}+1\right)$-normal.) This determines $\mathcal{X}^{\alpha+1}$ and $<^{\mathcal{X} / \mathcal{T}} \uparrow(\alpha+2)$; note that because $\Sigma$ has inflation condensation, $\mathcal{X}^{\alpha+1}$ is in fact via $\Sigma$, and in particular has wellfounded models. It just remains to define $\varrho_{\alpha+1}$ and prove $\varphi(\alpha+2)$.

Let $\kappa_{E}=\operatorname{cr}(E)$ and $\kappa_{F}=\varrho_{\alpha}\left(\kappa_{E}\right)=\operatorname{cr}(F)$. Let $\beta=\operatorname{pred}^{\mathcal{U}}(\alpha+1)$ and $\xi=\operatorname{pred}^{\mathcal{X}^{\alpha+1}}\left(\zeta^{\alpha}+1\right)$. So for all $\gamma<\beta$, we have $\widetilde{\nu}_{\gamma}^{\mathcal{U}} \leq \kappa_{E}<\widetilde{\nu}_{\beta}^{\mathcal{U}}$, so by N5,

$$
\widetilde{\nu}_{\zeta^{\gamma}}^{\mathcal{X}^{\alpha+1}}=\varrho_{\alpha}\left(\widetilde{\nu}_{\gamma}^{\mathcal{U}}\right) \leq \kappa_{F}<\varrho_{\alpha}\left(\widetilde{\nu}_{\beta}^{\mathcal{U}}\right)=\widetilde{\nu}_{\zeta^{\beta}}^{\mathcal{X}^{\alpha+1}}
$$

so $\xi \in\left[\lambda^{\beta}, \zeta^{\beta}\right]$. Therefore $<^{\mathcal{U}} \uparrow(\alpha+2)=<^{\mathcal{X} / \mathcal{T}} \uparrow(\alpha+2)$, giving N2.

For the remaining properties we split into cases.

SucCessor Case $1 . \beta$ is easy or $\mathcal{U}$ drops in model or degree at $\alpha+1$.

The overall argument here is routine and left to the reader. However, there are a couple of details which are new, and which we discuss.

We first show that $\alpha+1$ is easy (and establish some other useful facts). If $\beta$ is easy this is immediate. Suppose $\beta$ is non-easy but $\alpha+1 \in \mathscr{D}^{\mathcal{U}}$. So $E_{\beta}^{\mathcal{U}} \neq F^{M_{\beta}^{\mathcal{U}}}$, and in fact

$$
\kappa_{E}<\operatorname{ind}\left(E_{\beta}^{\mathcal{U}}\right)<\left(\kappa_{E}^{+}\right)^{M_{\beta}^{\mathcal{U}}},
$$

and as $\varrho_{\beta}$ is nice,

$$
\kappa_{F}<\operatorname{ind}\left(E_{\zeta^{\beta}}^{\mathcal{X}^{\alpha+1}}\right)<\left(\kappa_{F}^{+}\right)^{M_{\infty}^{\mathcal{X}^{\beta}}} .
$$

Now $\xi=\zeta^{\beta}$. For otherwise $\xi \in\left[\lambda^{\beta}, \zeta^{\beta}\right)$ and $\kappa_{F}<\operatorname{ind}\left(E_{\xi}^{\mathcal{X}^{\beta}}\right)$. But $\operatorname{ind}\left(E_{\xi}^{\mathcal{X}^{\beta}}\right)$ is a cardinal in $M_{\infty}^{\mathcal{X}^{\beta}}$, and so $\left(\kappa_{F}^{+}\right)^{M_{\beta}^{\mathcal{u}}}=\left(\kappa_{F}^{+}\right)^{\operatorname{ex}_{\xi}^{\mathcal{X}^{\beta}}} \leq \operatorname{ind}\left(E_{\zeta^{\beta}}^{\mathcal{X}^{\alpha+1}}\right)$, contradiction. Similarly,

$$
\begin{gathered}
M_{\zeta^{\alpha}+1}^{* \mathcal{X}^{\alpha+1}}=\varrho_{\beta}\left(N_{\alpha+1}^{*}\right) \triangleleft M_{\zeta^{\beta}}^{\mathcal{X}^{\beta}}=M_{\zeta^{\beta}}^{\mathcal{X}^{\alpha+1}}, \\
\text { and if } \zeta^{\beta}+1<\operatorname{lh}\left(\mathcal{X}^{\beta}\right) \text { then } M_{\zeta^{\alpha}+1}^{* \mathcal{X}^{\alpha+1}} \triangleleft \operatorname{ex}_{\zeta^{\beta}}^{\mathcal{X}^{\beta}},
\end{gathered}
$$

(for the latter, use the fact that $\operatorname{ind}\left(E_{\zeta^{\beta}}^{\mathcal{X}^{\alpha+1}}\right)<\operatorname{ind}\left(E_{\zeta^{\beta}}^{\mathcal{X}^{\beta}}\right)$ and $\operatorname{ind}\left(E_{\zeta^{\beta}}^{\mathcal{X}^{\beta}}\right)$ is a cardinal of $M_{\infty}^{\mathcal{X}^{\beta}}$ ), so $\zeta^{\alpha}+1 \in \mathscr{D}^{\mathcal{X}^{\alpha+1}}$ and $\lambda^{\alpha+1} \notin C^{\alpha+1}$, hence $\alpha+1$ is easy.

Now suppose that $\beta$ is non-easy but $\mathcal{U}$ drops in degree, but not in model, at $\alpha+1$. Then we claim that $\xi+1=\operatorname{lh}\left(\mathcal{X}^{\beta}\right)$, and therefore $\alpha+1$ is easy (but $\lambda^{\alpha+1} \in C^{\alpha+1}$ ). For because $\beta$ is non-easy, we have $[0, \beta]_{\mathcal{U}} \cap \mathscr{D}_{\operatorname{deg}}^{\mathcal{U}}=\emptyset$ by property N3, so

$$
n=\mathrm{u}-\operatorname{deg}^{\mathcal{U}}(\beta)=\mathrm{u}-\operatorname{deg}^{\mathcal{T}}(\infty) .
$$

So $\mathrm{u}-\rho_{n}\left(M_{\beta}^{\mathcal{U}}\right) \leq \kappa_{E}$. Letting $\varepsilon+1 \in b^{\mathcal{T}}$ and $G=E_{\varepsilon}^{\mathcal{T}}$, then we have $\operatorname{ind}(G) \leq$ $\mathrm{u}-\rho_{n}\left(M_{\infty}^{\mathcal{T}}\right)$, and $i_{0 \beta}^{\mathcal{U}}$ is continuous at $\operatorname{ind}(G)$, so $i_{0 \beta}^{\mathcal{U}}(\operatorname{ind}(G))<\kappa_{E}$. But by properties N7 and N5 we have

$$
\varrho_{\beta} \circ i_{0 \beta}^{\mathcal{U}}(\operatorname{ind}(G))=\omega_{\infty}^{\beta}(\operatorname{ind}(G)) \geq \operatorname{ind}\left(E_{\delta_{\varepsilon}^{\beta}}^{\mathcal{X}^{\beta}}\right) .
$$

Therefore $\varrho_{\beta}(\kappa)>\operatorname{ind}\left(E_{\delta_{\varepsilon}^{\beta}}^{\mathcal{X}^{\beta}}\right)$. This holds for every $\varepsilon+1 \in b^{\mathcal{T}}$, and it follows that $\xi+1=\ln \left(\mathcal{X}^{\beta}\right)$. 
To see that if $\mathcal{T}$ is terminally non-dropping and $[0, \alpha+1]_{\mathcal{U}}$ does not drop in model or degree, then $\varrho_{\alpha+1}$ is a $\mathrm{u}$-m-embedding, use the cofinality of the relevant maps at $\mathrm{u}-\rho_{m}$.

We now consider the verification that $\varrho_{\alpha+1}$ is a near $\mathrm{u}$ - $k$-embedding, where $k=\mathrm{u}$ - $\operatorname{deg}^{\mathcal{U}}(\alpha+1)$, given that $[0, \alpha+1]_{\mathcal{U}} \cap \mathscr{D}_{\text {deg }}^{\mathcal{U}} \neq \emptyset$. The reader can safely skip this proof on a first pass, if they are so inclined, moving to Case 2 below; it is just a detail which is not central to our considerations. We officially assume that $M$ is $\lambda$-indexed for the proof, and thus can drop the prefix "u-". The proof is mostly like in that of Lemma 4.20 (which was a slight variant of that in [9]), so we leave most of the details to the reader. However, it requires one extra observation. Fix $\delta \leq \mathcal{U} \beta$ largest such that $[0, \delta]_{\mathcal{U}}$ does not drop in model or degree. So $\operatorname{deg}^{\mathcal{U}}(\delta)=n$ and $\varrho_{\delta}: M_{\delta}^{\mathcal{U}} \rightarrow M_{\infty}^{\mathcal{X}^{\delta}}$ is an $n$-lifting embedding. Let

$$
X=\left\{\gamma \leq \alpha+1 \mid \delta<^{\mathcal{U}} \gamma \text { and } \operatorname{succ}^{\mathcal{U}}(\delta, \gamma) \in \mathscr{D}_{\mathrm{deg}}^{\mathcal{U}}\right\}
$$

and $X^{\prime}=\left\{\lambda^{\gamma} \mid \gamma \in X\right\}$. Note that $\gamma \mapsto \lambda^{\gamma}$ is an isomorphism between $<^{\mathcal{U}}\lceil X$ and $<^{\mathcal{X}^{\alpha+1}} \mid X^{\prime}$. For $\chi$ such that $\chi+1 \in X$, we define strong closeness at $\chi$ (relating the definability of the measures of $E_{\chi}^{\mathcal{U}}$ to that of their lifts, measures of $E_{\zeta^{\chi}}^{\mathcal{X}^{\alpha+1}}$ ), and for $\varepsilon \in X$, we define translatability at $\varepsilon$ (which, given $\gamma+1 \in X$ with $\gamma+1 \leq \mathcal{U} \varepsilon$ and $(\gamma+1, \varepsilon) \mathcal{U} \cap \mathscr{D}^{\mathcal{U}}=\emptyset$, allows us to translate definitions of subsets of $\operatorname{cr}\left(i_{\gamma+1, \varepsilon}^{* \mathcal{U}}\right)$ over $M_{\varepsilon}^{\mathcal{U}}$, to definitions over $M_{\gamma+1}^{* \mathcal{U}}$, in a manner which reflects up to $M_{\lambda^{\varepsilon}}^{\mathcal{X}^{\alpha+1}}$ and $M_{\lambda \gamma+1}^{* \mathcal{X}^{\alpha+1}}$ ). One proves these properties hold inductively, basically as in [9] (simultaneously showing that $\varrho_{\gamma}$ is a near $\operatorname{deg}^{\mathcal{U}}(\gamma)$-embedding for each $\left.\gamma \in X\right)$. However, there is a wrinkle in verifying that $\varrho_{\alpha+1}$ is a near $k$-embedding for example when:

$$
\begin{aligned}
& -[0, \alpha+1]_{\mathcal{U}} \cap \mathscr{D}^{\mathcal{U}}=\emptyset \text { (but }[0, \alpha+1]_{\mathcal{U}} \cap \mathscr{D}_{\text {deg }}^{\mathcal{U}} \neq \emptyset, \text { so } \xi+1=\operatorname{lh}\left(\mathcal{X}^{\beta}\right) \text { and } \\
& \left.\lambda^{\alpha+1} \notin \mathscr{D}^{\mathcal{X}^{\alpha+1}} \text { and } M_{\lambda^{\alpha+1}}^{* \mathcal{X}^{\alpha+1}}=M_{\xi}^{\mathcal{X}^{\beta}}=M_{\infty}^{\mathcal{X}^{\beta}}\right), \\
& -k+1=n\left(\text { so } \rho_{k+1}^{M_{\mathcal{\beta}}^{\mathcal{U}}} \leq \kappa_{E}<\rho_{k}^{M_{\beta}^{\mathcal{U}}} \text { where } E=E_{\alpha}^{\mathcal{U}}\right), \\
& -\operatorname{deg}^{\mathcal{X}^{\alpha+1}}\left(\lambda^{\alpha+1}\right)=k+1\left(\text { so } \varrho_{\beta}\left(\rho_{k+1}^{M_{\beta}^{\mathcal{U}}}\right) \leq \varrho_{\beta}\left(\kappa_{E}\right)<\rho_{k+1}\left(M_{\xi}^{\mathcal{X}^{\beta}}\right)\right) .
\end{aligned}
$$

For $i \in\{k, k+1\}$ let $U_{i}=\operatorname{Ult}_{i}\left(M_{\xi}^{\mathcal{X}^{\beta}}, F\right)$ where $F=E_{\zeta^{\alpha}}^{\mathcal{X}^{\alpha+1}}$. Let $j_{i}: M_{\xi}^{\mathcal{X}^{\beta}} \rightarrow U_{i}$ be the ultrapower map. By induction, $\varrho_{\beta}$ is a near $k$-embedding, and letting

$$
\bar{\varrho}: M_{\alpha+1}^{\mathcal{U}}=\operatorname{Ult}_{k}\left(M_{\beta}^{\mathcal{U}}, E_{\alpha}^{\mathcal{U}}\right) \rightarrow U_{k}
$$

be given by the Shift Lemma, then as above (using the argument of [9]), $\varrho$ is a near $k$-embedding. Now we have $\operatorname{deg}^{\mathcal{X}^{\alpha+1}}\left(\lambda^{\alpha+1}\right)=k+1$, and $\varrho_{\alpha+1}=\varrho^{\prime} \circ \varrho$ where $\varrho^{\prime}: U_{k} \rightarrow U_{k+1}$ is the natural factor map. So it suffices to see that, in fact, $U_{k}=U_{k+1}$ and $\varrho^{\prime}=$ id; this completes the proof.

To see this, it suffices to see that $\varrho^{\prime}$ " $\rho_{k}^{U_{k}}$ is cofinal in $\rho_{k}^{U_{k+1}}$. For suppose this holds. Note $\varrho^{\prime}\left(\vec{p}_{k+1}^{U_{k}}\right)=\vec{p}_{k+1}^{U_{k+1}}$, and by [18, Lemma 2.4], it follows that $\varrho^{\prime}$ is a $k$ embedding. But $U_{k}, U_{k+1}$ are $(k+1)$-sound (see the proof of [12, Corollary 2.24], 
for example) and $\rho_{k+1}^{U_{k}}=\rho_{k+1}^{U_{k+1}}$ and $\varrho^{\prime}\left\lceil\rho_{k+1}^{U_{k}}=\right.$ id. It follows that $U_{k+1} \subseteq \operatorname{rg}\left(\varrho^{\prime}\right)$, which suffices.

To see the desired cofinality of $\varrho^{\prime}$, it suffices to see that $j_{k+1}$ is continuous at $\rho_{k}\left(M_{\xi}^{\mathcal{X}^{\beta}}\right)$, since $j_{k+1}=\varrho^{\prime} \circ j_{k}$ and $\rho_{k}^{U_{k}}=\sup j_{k} " \rho_{k}\left(M_{\xi}^{\mathcal{X}^{\beta}}\right)$.

Now let $\mu^{\mathcal{X}^{\beta}}=\operatorname{wcof}_{k+1}^{M_{\xi}^{\mathcal{X}^{\beta}}}$ (see Definition 9.9). We have $\operatorname{deg} \mathcal{X}^{\alpha+1}\left(\lambda^{\alpha+1}\right)=$ $k+1$, so $\operatorname{cr}(F)<\rho_{k+1}\left(M_{\xi}^{\mathcal{X}^{\beta}}\right)$. So $j_{k+1}$ is continuous at $\rho_{k}\left(M_{\xi}^{\mathcal{X}^{\beta}}\right)$ iff $\operatorname{cr}(F) \neq \mu^{\mathcal{X}^{\beta}}$. So we must see $\operatorname{cr}(F) \neq \mu^{\mathcal{X}^{\beta}}$.

Let $\mu^{\mathcal{T}}=\operatorname{wcof}_{k+1}^{M_{\infty}^{\mathcal{T}}}$. Then because $\psi_{0 \beta}=\omega_{\infty}^{0 \beta}$ is a near $(k+1)$-embedding (as $\left.\operatorname{deg}^{\mathcal{T}}(\infty)=k+1=\operatorname{deg}^{\mathcal{X}^{\alpha+1}}\left(\lambda^{\alpha+1}\right)=\operatorname{deg}^{\mathcal{X}^{\beta}}(\xi)\right)$ and by Lemma 9.10, either:

$-\mu^{\mathcal{T}}<\rho_{k+1}\left(M_{\infty}^{\mathcal{T}}\right)$ and $\mu^{\mathcal{X}^{\beta}}=\psi_{0 \beta}\left(\mu^{\mathcal{T}}\right)$, or

$-\mu^{\mathcal{T}}=\rho_{k+1}\left(M_{\infty}^{\mathcal{T}}\right)$ and $\mu^{\mathcal{X}^{\beta}}=\rho_{k+1}\left(M_{\xi}^{\mathcal{X}^{\beta}}\right)$.

But $\operatorname{cr}(F)<\rho_{k+1}\left(M_{\xi}^{\mathcal{X}^{\beta}}\right)$. So suppose $\mu^{\mathcal{T}}<\rho_{k+1}\left(M_{\infty}^{\mathcal{T}}\right)$ and (by commutativity)

$$
\mu^{\mathcal{X}^{\beta}}=\psi_{0 \beta}\left(\mu^{\mathcal{T}}\right)=\varrho_{\beta}\left(i_{0 \beta}^{\mathcal{U}}\left(\mu^{\mathcal{T}}\right)\right) .
$$

Then since $\operatorname{deg}^{\mathcal{U}}(0)=\operatorname{deg}^{\mathcal{U}}(\beta)=k+1$ and $\operatorname{deg}^{\mathcal{U}}(\alpha+1)=k$,

$$
i_{0 \beta}^{\mathcal{U}}\left(\mu^{\mathcal{T}}\right)<\rho_{k+1}\left(M_{\beta}^{\mathcal{U}}\right) \leq \operatorname{cr}(E)
$$

so $\mu^{\mathcal{X}^{\beta}}<\varrho_{\beta}(\operatorname{cr}(E))=\operatorname{cr}(F)$, completing the proof that $\varrho_{\alpha+1}$ is a near $k$ embedding.

We do not need this kind of argument for degrees $<k$, because if $\varrho: R \rightarrow S$ is a near $k$-embedding where $k>0$, then $\varrho\left(\rho_{k}^{R}\right) \geq \rho_{k}^{S}$. We leave the remaining details in this case to the reader.

SuCCESSOR CASE 2. $\beta$ is non-easy, and $\mathcal{U}$ does not drop in model or degree at $\alpha+1$.

So $\xi \in C^{\beta}$. Let $\theta^{*}=f^{\beta}(\xi)$.

SUBCASE 2.1. $\xi+1=\ln \left(\mathcal{X}^{\beta}\right)$.

Then $\varrho_{\beta}: M_{\beta}^{\mathcal{U}} \rightarrow M_{\xi}^{\mathcal{X}^{\beta}}$, and everything is routine. We have $\lambda^{\alpha+1} \in C^{\alpha+1}$ but $\theta^{*}+1=\theta^{\alpha+1}+1=\operatorname{lh}(\mathcal{T})$, so $\alpha+1$ is easy.

SubCASE 2.2. $\xi+1<\ln \left(\mathcal{X}^{\beta}\right)$.

So $\theta^{*}+1<\operatorname{lh}(\mathcal{T})$. Now $E$ is total over $M_{\beta}^{\mathcal{U}}$ and $\left(\kappa_{E}^{+}\right)^{M_{\beta}^{\mathcal{U}}} \leq \widetilde{\nu}_{\beta}^{\mathcal{U}}$ (for $\kappa_{E}<\widetilde{\nu}_{\beta}^{\mathcal{U}}$, so if $\left(\kappa_{E}^{+}\right)^{M_{\beta}^{\mathcal{U}}}>\widetilde{\nu}_{\beta}^{\mathcal{U}}$ then $E_{\beta}^{\mathcal{U}}=F^{M_{\beta}^{\mathcal{U}}}$ and $\kappa_{E}=\operatorname{lgcd}\left(M_{\beta}^{\mathcal{U}}\right)$, but then $E_{\zeta^{\beta}}^{\mathcal{X}^{\beta}}=$ $F\left(M_{\infty}^{\mathcal{X}^{\beta}}\right)$ and $\kappa_{F}=\operatorname{lgcd}\left(M_{\infty}^{\mathcal{X}^{\beta}}\right)$, so $\xi+1=\operatorname{lh}\left(\mathcal{X}^{\beta}\right)$, contradiction). Therefore $\left(\kappa_{F}^{+}\right)^{M_{\infty}^{\mathcal{X}^{\beta}}} \leq \widetilde{\nu}_{\zeta^{\beta}}^{\mathcal{X}^{\alpha+1}}$, so $F$ is total over $M_{\infty}^{\mathcal{X}^{\beta}}$, so $F$ is total over $\operatorname{ex}_{\xi}^{\mathcal{X}^{\beta}}$ (and $E_{\xi}^{\mathcal{X}^{\beta}}$ is the copy of $E_{\theta^{*}}^{\mathcal{T}}$ ), so $\operatorname{ex}_{\xi}^{\beta} \unlhd M_{\zeta^{\alpha}+1}^{* \mathcal{X}^{\alpha+1}}$. So $\lambda^{\alpha+1}=\zeta^{\alpha}+1 \in C^{\alpha+1}$ and $\theta^{\alpha+1}=\theta^{*}$.

See Figure 7. Let $\psi=\psi_{\beta, \alpha+1}$ (see N7) and

$$
\varsigma=\omega_{\theta^{\alpha+1}}^{\beta, \alpha+1}=i_{\zeta^{\alpha}+1}^{* \mathcal{X}^{\alpha+1}}\left\lceil Q_{\xi}^{\beta} .\right.
$$




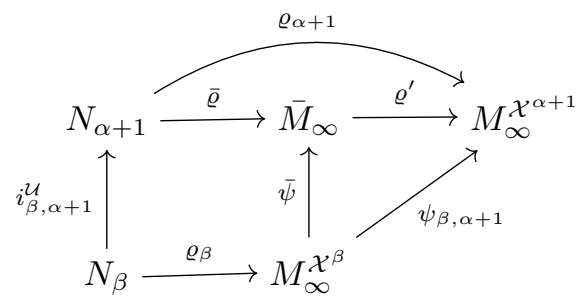

Figure 7: The diagram commutes, in Subcase 2.2.

By 8.11 (and recall property T3), $\psi$ is a near $\mathrm{u}$ - $n$-embedding, and by 8.13 , $\varsigma \uparrow Q_{\xi}^{\beta} \subseteq \psi$. So $F \uparrow \widetilde{\nu}(F)$ is the $\left(\kappa_{F}, \widetilde{\nu}(F)\right)$-extender derived from $\psi$, and note

$$
Q_{\xi}^{\beta}\left\|\left(\kappa_{F}^{+}\right)^{Q_{\xi}^{\beta}}=M_{\infty}^{\mathcal{X}^{\beta}}\right\|\left(\kappa_{F}^{+}\right)^{M_{\infty}^{\mathcal{X}^{\beta}}} .
$$

Let $\bar{M}_{\infty}, \bar{\psi}, \bar{\varrho}, \varrho^{\prime}, \varrho_{\alpha+1}$ be defined as follows:

$-\bar{M}_{\infty}=\operatorname{Ult}_{\mathrm{u}-n}\left(M_{\infty}^{\mathcal{X}^{\beta}}, F\right)$,

$-\bar{\psi}: M_{\infty}^{\mathcal{X}^{\beta}} \rightarrow \bar{M}_{\infty}$ is the associated ultrapower map $i_{F}^{M_{\infty}^{\mathcal{X}^{\beta}}, \mathrm{u}-n}$,

- $\bar{\varrho}: M_{\alpha+1}^{\mathcal{U}} \rightarrow \bar{M}_{\infty}$ is given by the Shift Lemma from $\varrho_{\beta}$ and $\varrho_{\alpha}\left\lceil\operatorname{ex}_{\alpha}^{\mathcal{U}}\right.$,

- $\varrho^{\prime}: \bar{M}_{\infty} \rightarrow M_{\infty}^{\mathcal{X}^{\alpha+1}}$ is the natural factor map (for example if $n=0$ then

$$
\varrho^{\prime}\left([a, f]_{F}^{M_{\infty}^{\mathcal{X}^{\beta}}}\right)=\psi(f)(a)
$$

and if $n>0$ use the obvious generalization; this is well-defined by the remarks above),

$-\varrho_{\alpha+1}=\varrho^{\prime} \circ \varrho \bar{\varrho}: M_{\alpha+1}^{\mathcal{U}} \rightarrow M_{\infty}^{\mathcal{X}^{\alpha+1}}$.

Let $\mu=i_{F}\left(\kappa_{F}\right)$. Then $\varrho^{\prime}$ is nice $\mathrm{u}-n$-lifting, $\varrho^{\prime} \circ \bar{\psi}=\psi$, and

$$
\operatorname{cr}\left(\varrho^{\prime}\right)>\left(\mu^{+}\right)^{\bar{M}_{\infty}}=\left(\mu^{+}\right)^{M_{\infty}^{\mathcal{X}^{\alpha+1}}},
$$

so $\varrho^{\prime}$ fixes $\widetilde{\nu}_{\zeta^{\alpha}}^{\mathcal{X}^{\alpha+1}}$ and $\operatorname{ind}\left(E_{\zeta^{\alpha}}^{\mathcal{X}^{\alpha+1}}\right)=\operatorname{ind}(F)$. (The latter holds as $\varrho^{\prime}\lceil\mu=\operatorname{id}$ and $\psi\left(\kappa_{F}\right)=\mu$ and $\left(\mu^{+}\right)^{\bar{M}_{\infty}}=\left(\mu^{+}\right)^{Q_{\theta^{\alpha+1}}^{\alpha+1}}=\left(\mu^{+}\right)^{M_{\infty}^{\mathcal{X}^{\alpha+1}}}$.)

Also note that $\varrho$ is nice $\mathrm{u}$ - $n$-lifting, $\varrho_{\alpha}\left\lceil\operatorname{ex}_{\alpha}^{\mathcal{U}} \subseteq \bar{\varrho}, \bar{\varrho}\left(\widetilde{\nu}_{\alpha}^{\mathcal{U}}\right)=\left(\widetilde{\nu}_{\zeta^{\alpha}}^{\mathcal{X}^{\alpha+1}}\right), \bar{\varrho}(\operatorname{ind}(E))=\right.$ $\operatorname{ind}(F)$ ), and $\bar{\varrho} \circ i_{\beta, \alpha+1}^{\mathcal{U}}=\bar{\psi} \circ \varrho_{\beta}$. (We have ind $(E) \in M_{\alpha+1}^{\mathcal{U}}$ because if $\operatorname{ind}(E)=$ $\mathrm{OR}^{M_{\alpha+1}^{\mathcal{U}}}$, so $\kappa_{E}=\operatorname{lgcd}\left(M_{\beta}^{\mathcal{U}}\right)$ and $M_{\beta}^{\mathcal{U}}$ is active type 2 , then $\kappa_{F}=\operatorname{lgcd}\left(M_{\infty}^{\mathcal{X}^{\beta}}\right)$, but then $\xi+1=\ln \left(\mathcal{X}^{\beta}\right)$, contradiction).

Therefore

- $\varrho_{\alpha+1}$ is nice $\mathrm{u}-n$-lifting, 


$$
\begin{aligned}
& -\varrho_{\alpha}\left\lceil\operatorname{ex}_{\alpha}^{\mathcal{U}} \subseteq \varrho_{\alpha+1},\right. \\
& \left.-\varrho_{\alpha+1}\left(\widetilde{\nu}_{\alpha}^{\mathcal{U}}\right)=\widetilde{\nu}_{\zeta^{\alpha}}^{\mathcal{X}^{\alpha+1}} \text { and } \varrho_{\alpha+1}\left(\operatorname{ind}\left(E_{\alpha}^{\mathcal{U}}\right)\right)=\operatorname{ind}\left(E_{\zeta^{\alpha}}^{\mathcal{X}^{\alpha+1}}\right)\right), \\
& -\psi_{\beta, \alpha+1} \circ \varrho_{\beta}=\varrho_{\alpha+1} \circ i_{\beta, \alpha+1}^{\mathcal{U}} .
\end{aligned}
$$

It is now easy to see that $\varphi(\alpha+2)$ holds.

Now suppose $\eta<\Omega$ is a limit. We have

$$
\mathcal{X}^{\eta} \uparrow \lambda^{\eta}=\bigcup_{\alpha<\eta} \mathcal{X}^{\alpha} \uparrow\left(\zeta^{\alpha}+1\right)
$$

and $\left[0, \lambda^{\eta}\right)_{\mathcal{X} \eta}=\Sigma\left(\mathcal{X}^{\eta}\left\lceil\lambda^{\eta}\right)\right.$, giving $\mathcal{X}^{\eta} \uparrow\left(\lambda^{\eta}+1\right)$. Since $<^{\mathcal{U}}\left\lceil\eta=\left(<^{\mathcal{X}^{\eta}} / \mathcal{T}\right)\lceil\eta\right.$, we can and do define a $\mathcal{U}\lceil\eta$-cofinal branch by setting

$$
[0, \eta)_{\mathcal{U}}=[0, \eta)_{\mathcal{X} \eta} / \mathcal{T},
$$

maintaining property N2. Note that $\mathcal{X}^{\eta}$ exists (and is according to $\Sigma$ ), by inflation condensation and as $\eta<\Omega$. We now define

$$
\varrho_{\eta}: M_{\eta}^{\mathcal{U}} \rightarrow M_{\infty}^{\mathcal{X}^{\eta}}
$$

it will then be easy to see that $\varphi(\eta+1)$ holds.

Limit CASE 1 . There is $\alpha<\eta$ such that $\alpha$ is easy and $\lambda^{\alpha}<^{\mathcal{X}^{\eta}} \lambda^{\eta}$.

Then $\beta$ is easy for every $\beta \in[\alpha, \eta]_{\mathcal{X}^{\eta} / \mathcal{T}}$, and using the inductive hypotheses, $[0, \eta)_{\mathcal{U}}$ has only finitely many drops and we can define $\varrho_{\eta}$ commuting with earlier maps in a routine manner.

Limit CASE 2. Otherwise. ${ }^{59}$

By 8.13, $\psi_{\alpha \eta}=\psi_{\beta \eta} \circ \psi_{\alpha \beta}$ for all $\alpha \leq^{\mathcal{U}} \beta<^{\mathcal{U}} \eta$. So by the commutativity given by property N7 we can and do define $\varrho_{\eta}$ in a unique manner preserving commutativity. That is,

$$
\varrho_{\eta} \circ i_{\alpha \eta}^{\mathcal{U}}=\psi_{\alpha \eta} \circ \varrho_{\alpha}
$$

for all $\alpha<^{\mathcal{U}} \eta$.

This completes the definition of $\Upsilon_{\mathcal{T}}^{\Sigma}$; clearly it is a $(\mathrm{u}-n, \Omega)$-strategy for $N$, as desired. If $\ln (\mathcal{U})=\alpha+1$ then we finally set $\mathcal{X}=\mathcal{X}^{\alpha}$, and if $\operatorname{lh}(\mathcal{U})$ is a limit $\eta$ we set $\mathcal{X}=\bigcup_{\alpha<\eta} \mathcal{X}^{\alpha} \uparrow\left(\lambda^{\alpha}+1\right)$. So if $\ln (\mathcal{U})$ is a successor then $\mathcal{X}$ is a $\mathcal{T}$-terminal inflation of $\mathcal{T}$.

Finally suppose that $\Sigma$ extends to a (u- $m, \Omega+1)$-strategy $\Sigma^{\prime}$ for $M$. Then $\Upsilon_{\mathcal{T}}^{\Sigma}$ extends to a $(\mathrm{u}-n, \Omega+1)$-strategy $\Upsilon_{\mathcal{T}}^{\Sigma^{\prime}}$ for $N$. For given $\mathcal{U}$ via $\Upsilon_{\mathcal{T}}^{\Sigma}$ of length $\Omega$, note that $\mathcal{X}$ also has length $\Omega$, and $\varphi(\Omega)$ holds. But then just as in the limit case above, we get a $\mathcal{U}$-cofinal branch $b$, and $M_{b}^{\mathcal{U}}$ is well-defined and wellfounded as $\operatorname{cof}(\Omega)>\omega$, so player II has won. We don't actually need $\mathcal{X}^{\Omega}$ here, but we can and do define it by copying the remainder of $\mathcal{T}$ following $\mathcal{X}^{\Omega} \uparrow(\Omega+1)$. Of course if $\ln \left(\mathcal{X}^{\Omega}\right)>\Omega+1$ then $\mathcal{X}^{\Omega}$ is not literally via $\Sigma$, but note that its models are wellfounded, because $\Omega>\omega$ is regular. We then define $\psi_{\alpha \Omega}$ and $\varrho_{\Omega}$ as before.

\footnotetext{
${ }^{59}$ In this case, $\eta$ itself can be easy, but this is not relevant.
} 
9.11 Definition. Given the objects above, let

$$
\mathcal{W}_{\mathcal{T}}^{\Sigma}(\mathcal{U})=\mathcal{W}^{\Sigma}(\mathcal{T}, \mathcal{U})=\mathcal{X}
$$

and if $\ln (\mathcal{U})$ is also a successor, let

$$
\varrho_{\mathcal{T}}^{\Sigma}(\mathcal{U})=\varrho^{\Sigma}(\mathcal{T}, \mathcal{U})=\varrho_{\operatorname{lh}(\mathcal{U})-1}
$$

And $\Upsilon_{\mathcal{T}}^{\Sigma}$ denotes the $\mathrm{u}$ - $\operatorname{deg}^{\mathcal{T}}(\infty)$-strategy for $N=M_{\infty}^{\mathcal{T}}$ defined above.

9.12 Remark. The following observation, which is natural, but not actually important for our construction, is mostly due to Steel: ${ }^{60}$ One could actually drop the superscript " $\Sigma$ " in the notation $\mathcal{W}_{\mathcal{T}}^{\Sigma}$ and $\varrho_{\mathcal{T}}^{\Sigma}$, without ambiguity.

For consider the limit stage $\eta$ in the preceding construction. Let $\widetilde{\mathcal{X}}=\mathcal{X}\left\lceil\lambda^{\eta}\right.$ be defined as above. We observe that $[0, \eta)_{\mathcal{U}}$ determines $\left[0, \lambda_{\eta}\right)_{\mathcal{X}^{\eta}}$, subject to the requirement that $\mathcal{X}^{\eta}$ be an inflation of $\mathcal{T}$. In fact, for any $\mathcal{U}\lceil\eta$-cofinal branch $b$ there is a unique $\widetilde{\mathcal{X}}$-cofinal branch $c=c_{b}$ such that

$-c$ induces $b$ in the same manner that $\left[0, \lambda^{\eta}\right)_{\mathcal{X}} \eta$ induces $[0, \eta)_{\mathcal{U}}$, and

- if $b \cap \mathscr{D}^{\mathcal{U}}=\emptyset$ then $\widetilde{\mathcal{X}}^{\wedge} c$ is a putative inflation of $\mathcal{T}$, meaning that all requirements of inflations are met, excluding the requirement that $M_{c}^{\widetilde{\mathcal{X}}}$ be well-defined and wellfounded.

For write $C=C^{\mathcal{T} \rightsquigarrow \tilde{\mathcal{X}}}$, etc, and $C^{\prime}=C^{\mathcal{T} \rightsquigarrow(\tilde{\mathcal{X}}, c)}$, etc (for a candidate $c$ ). If $\lambda^{\beta} \notin C$ for some $\beta \in b$, this is immediate (and $\lambda^{\eta} \notin C^{\prime}$ ). So suppose otherwise and let $\theta=\sup _{\beta \in b} f\left(\lambda^{\beta}\right)$. Then $\lambda^{\eta} \in C^{\prime}$ and $f^{\prime}\left(\lambda^{\eta}\right)=\theta$. If $\theta=f\left(\lambda^{\beta}\right)$ for some $\beta \in b$ (hence $\theta=f\left(\lambda^{\beta}\right)$ for all sufficiently large $\beta \in b$ ) then everything is clear. So suppose otherwise; then $\theta$ is a limit. Note that for $c$ as desired to exist, we must have $\theta<\operatorname{lh}(\mathcal{T})$. Note that for $\alpha<\theta, \gamma_{\alpha}=\operatorname{def}_{\operatorname{dim}} \operatorname{lib}_{\beta} \gamma_{\alpha}^{\beta}$ exists, $c=\bigcup_{\alpha<\mathcal{T}_{\theta}}\left[0, \gamma_{\alpha}\right)_{\tilde{\mathcal{X}}}$ is an $\widetilde{\mathcal{X}}$-cofinal branch, $(\widetilde{\mathcal{X}}, c)$ is a putative inflation of $\mathcal{T}, c$ determines $b$, and moreover, $c$ is the unique such branch.

9.13 Remark. Consider condition N3 of the preceding construction. By this condition, $\varrho_{\alpha}$ is a $\mathrm{u}$ - $k$-lifting embedding, and if $[0, \alpha]_{\mathcal{U}} \cap \mathscr{D}_{\operatorname{deg}} \neq \emptyset$ then $\varrho_{\alpha}$ is a near $\mathrm{u}$ - $k$-embedding. Also by this condition, if $\mathcal{T}$ is terminally non-dropping and $[0, \alpha]_{\mathcal{U}} \cap \mathscr{D}_{\operatorname{deg}}^{\mathcal{U}}=\emptyset$ then $\varrho_{\alpha}$ is a near u- $k$-embedding (in fact a $\mathrm{u}$ - $k$-embedding). But $\varrho_{\alpha}$ can fail to be a near $\mathrm{u}-n$-embedding when $\mathcal{T}$ is terminally dropping and $[0, \alpha]_{\mathcal{U}} \cap \mathscr{D}_{\operatorname{deg}}^{\mathcal{U}}=\emptyset$. Moreover, it can also be that $M$ has $\lambda$-indexing and:

$$
-n=k+1=\operatorname{deg}^{\mathcal{T}}(\infty)>0,
$$

\footnotetext{
${ }^{60}$ Our construction uses only the fact that the branches of $\mathcal{X}$ determine those of $\mathcal{U}$, so Steel's observation is not important for us here, and the author did not initially consider it. It is, however, relevant to Steel's construction, as he proceeds in the other direction. After we had developed most of our construction, Steel pointed out that for each $(b, d)$ such that $b$ is a $\mathcal{U}\lceil\eta$-cofinal branch and $d$ is either a node in $\mathcal{T}$ or a $\mathcal{T}$-maximal branch, there is at most one corresponding $\widetilde{\mathcal{X}}$-cofinal branch $c_{b, d}$. The author later noticed that $b$ in fact determines $d$, given that we are seeking an inflation of $\mathcal{T}$.
} 
- $\rho_{k+1}\left(M_{\alpha}^{\mathcal{U}}\right)<\operatorname{OR}\left(M_{\alpha}^{\mathcal{U}}\right)$

$-\varrho_{\alpha}\left(\rho_{k+1}\left(M_{\alpha}^{\mathcal{U}}\right)\right)<\rho_{k+1}\left(M_{\infty}^{\mathcal{X}^{\alpha}}\right)$

- $M_{\alpha}^{\mathcal{U}}$ has a measurable $\gamma \geq \rho_{k+1}\left(M_{\alpha}^{\mathcal{U}}\right)$ such that $\varrho_{\alpha}(\gamma)<\rho_{k+1}\left(M_{\infty}^{\mathcal{X}^{\alpha}}\right)$,

- $E_{\alpha}^{\mathcal{U}}$ is $M_{\alpha}^{\mathcal{U}}$-total with $\operatorname{cr}\left(E_{\alpha}^{\mathcal{U}}\right)=\gamma$ and $\zeta^{\alpha}+1=\ln \left(\mathcal{X}^{\alpha}\right)$ (and $E_{\zeta^{\alpha}}^{\mathcal{X}^{\alpha}}$ is $M_{\infty}^{\mathcal{X}^{\alpha}}$-total), so

$$
\operatorname{deg}^{\mathcal{U}}(\alpha+1)=k \text { but } \operatorname{deg}^{\mathcal{X}^{\alpha+1}}(\infty)=k+1
$$

(but as we saw, even in this case, $\varrho_{\alpha+1}$ is a near $k$-embedding).

For here is an example, with $k=0$. Suppose that $M$ is $\lambda$-indexed, 2-sound and $\rho_{2}^{M}<\rho_{1}^{M}<\mathrm{OR}^{M}$ and $\operatorname{cof}^{M}\left(\rho_{1}^{M}\right)=\kappa$ where $\kappa<\rho_{2}^{M}$ is $M$-measurable and $\rho_{1}^{M}$ is a limit of $M$-measurables. Let $\mu \in\left[\rho_{2}^{M}, \rho_{1}^{M}\right)$ be $M$-measurable and $E \in \mathbb{E}^{M}$ be a measure on $\mu$. Let $\mathcal{T}$ be the 2-maximal tree on $M$ using only $E$. So $N=M_{1}^{\mathcal{T}}, n=\operatorname{deg}^{\mathcal{T}}(1)=1$, and $\mathcal{U}$ will be a 1-maximal tree. Let $F \in \mathbb{E}^{M} \cap \mathbb{E}^{N}$ be the order 0 measure on $\kappa$. Let $E_{0}^{\mathcal{U}}=F\left(\mathcal{U}\right.$ will use two extenders; $E_{1}^{\mathcal{U}}$ will be defined in a moment). This determines $\mathcal{X}^{0}=\mathcal{T}$ and $\mathcal{X}^{1}$. Note that (so far) there is no dropping in model in any of our trees. We have $\varrho_{1}: M_{1}^{\mathcal{U}} \rightarrow M_{\infty}^{\mathcal{X}^{1}}$. We claim $\operatorname{deg}^{\mathcal{U}}(1)=1=\operatorname{deg}^{\mathcal{X}^{1}}(\infty)$ but $\varrho_{1}\left(\rho_{1}\left(M_{1}^{\mathcal{U}}\right)\right)<\rho_{1}\left(M_{\infty}^{\mathcal{X}^{1}}\right)$, and therefore $\varrho_{1}$ is not a near 1 -embedding. To see this, use routine calculations to verify the following:

$$
\begin{aligned}
& -\operatorname{deg}^{\mathcal{T}}(1)=1=\operatorname{deg}^{\mathcal{U}}(0)=\operatorname{deg}^{\mathcal{U}}(1), \\
& -\lambda_{0}=\zeta_{0}=0\left(\operatorname{so} \lambda^{1}=1\right), \\
& -\ln \left(\mathcal{X}^{1}\right)=3 \text { and } E_{0}^{\mathcal{X}^{1}}=F \text { and } E_{1}^{\mathcal{X}^{1}}=i_{01}^{\mathcal{X}^{1}}(E), \\
& -\operatorname{deg}^{\mathcal{X}^{1}}(1)=2 \text { and } \operatorname{deg}^{\mathcal{X}^{1}}(2)=1, \\
& -\rho_{1}^{N}=\sup i^{\mathcal{T}} " \rho_{1}^{M}=i^{\mathcal{T}}\left(\rho_{1}^{M}\right), \\
& -\rho_{1}\left(M_{1}^{\mathcal{U}}\right)=\sup i_{01}^{\mathcal{U}} " \rho_{1}^{N}<i_{01}^{\mathcal{U}}\left(\rho_{1}^{N}\right)=i_{01}^{\mathcal{U}} \circ i^{\mathcal{T}}\left(\rho_{1}^{M}\right), \\
& -\sup i_{01}^{\mathcal{X}^{1}} " \rho_{1}^{M}<\rho_{1}\left(M_{1}^{\mathcal{X}^{1}}\right)=i_{01}^{\mathcal{X}^{1}}\left(\rho_{1}^{M}\right), \\
& -\rho_{1}\left(M_{2}^{\mathcal{X}^{1}}\right)=\sup i_{12}^{\mathcal{X}^{1}} " \rho_{1}\left(M_{1}^{\mathcal{X}^{1}}\right)=i_{12}^{\mathcal{X}^{1}}\left(\rho_{1}\left(M_{1}^{\mathcal{X}^{1}}\right)\right)=i_{02}^{\mathcal{X}^{1}}\left(\rho_{1}^{M}\right), \\
& -\varrho_{1} \circ i_{01}^{\mathcal{U}} \circ i^{\mathcal{T}}=i_{02}^{\mathcal{X}}, \text { and hence, } \varrho_{1}\left(i_{01}^{\mathcal{U}}\left(i^{\mathcal{T}}\left(\rho_{1}^{M}\right)\right)\right)=i_{02}^{\mathcal{X}}\left(\rho_{1}^{M}\right)=\rho_{1}\left(M_{2}^{\mathcal{X}^{1}}\right) .
\end{aligned}
$$

The claim follows from these facts, and gives the desired example. 


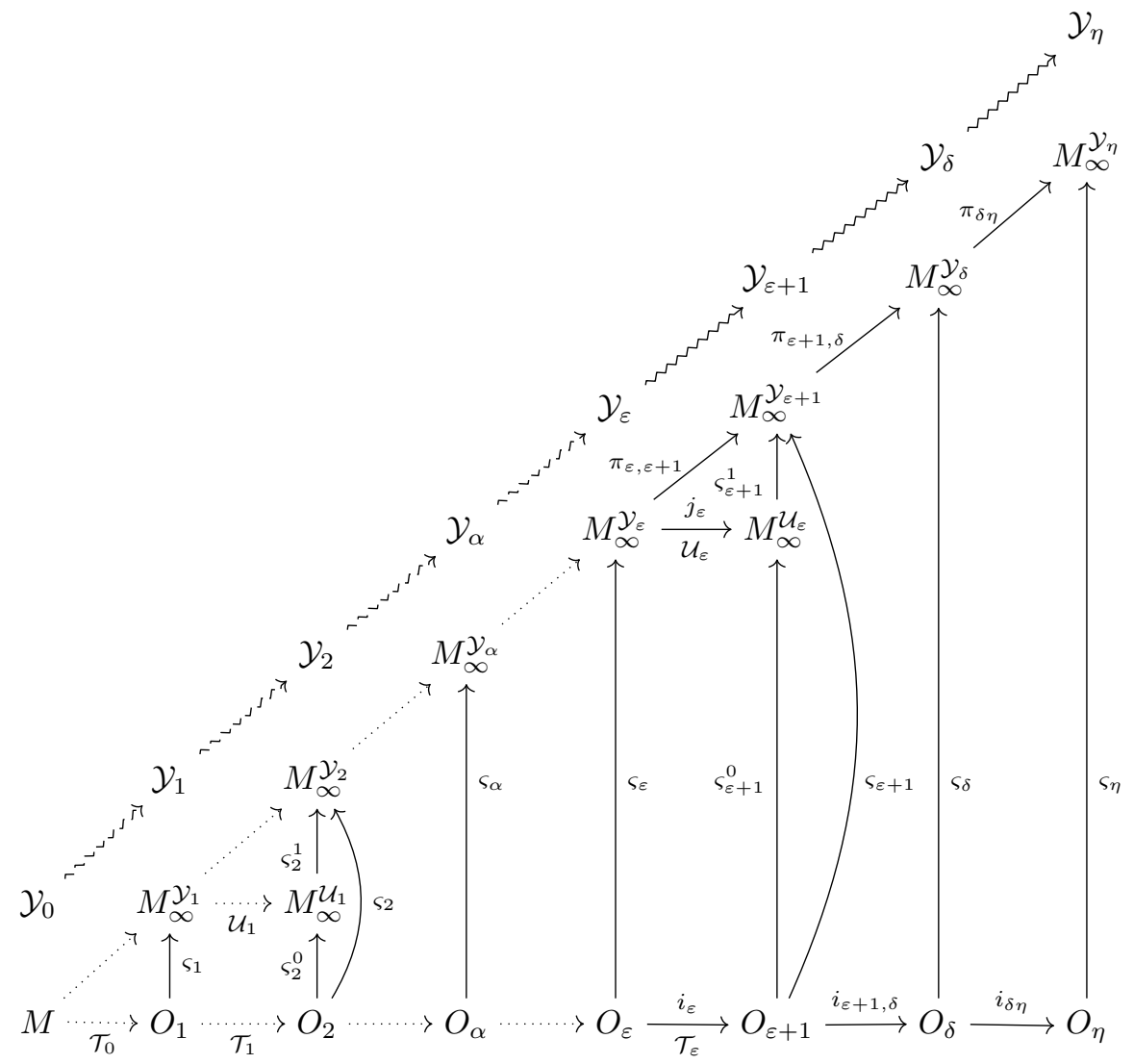

Figure 8: Commutative diagram for an infinite stack. Note that $\mathcal{U}_{\beta}, \varsigma_{\beta+1}^{0}, \varsigma_{\beta+1}^{1}$ are not mentioned in conditions S1-S10. Note that $\mathcal{Y}_{0}$ is trivial, $O_{2}=M_{\infty}^{\mathcal{T}_{1}}$ and $N_{\varepsilon+1}=M_{\infty}^{\mathcal{T}_{\varepsilon}}$. The squiggly arrows indicate inflations $\mathcal{Y} \rightsquigarrow \mathcal{Z}$; a dashed squiggly arrow indicates that $\mathcal{Z}$ is possibly $\mathcal{Y}$-terminally-model-dropping, whereas a solid squiggly arrow indicates that $\mathcal{Z}$ is $\mathcal{Y}$-terminally-non-model-dropping. The solid horizontal arrows are iteration maps; $i_{\beta \gamma}=i^{\vec{T}[\beta, \gamma}$ ) (and $b^{\vec{T}[\beta, \gamma)}$ does not drop in model where they appear in the diagram) $i_{\varepsilon}=i_{\varepsilon, \varepsilon+1}$ and $j_{\varepsilon}=i^{\mathcal{U}_{\varepsilon}}$. Dotted horizontal arrows represent iteration trees possibly dropping on their main branches. Solid diagonal arrows are final inflation copy maps $\pi_{\infty}^{\mathcal{Y} \rightsquigarrow \mathcal{Z}}$ (such exist where they appear in the diagram). Dotted diagonal arrows represent inflations $\mathcal{Y} \rightsquigarrow \mathcal{Z}$ which are possibly $\mathcal{Y}$-terminally-model-dropping. Vertical arrows are the lifting maps $\varsigma_{\delta}$. All solid arrows commute. 


\subsubsection{Stacks of limit length}

From now on we assume that $\Sigma$ is a $(\mathrm{u}-m, \Omega+1)$-strategy for $M$ with inflation condensation. We will define an optimal- $(\mathrm{u}-m, \Omega, \Omega+1)^{*}$-strategy $\Sigma^{*}$ for $M$. Let us say that a round of the iteration game consists of a single normal tree. Given $\alpha<\Omega$, at the start of round $\alpha$, with player II not yet having lost, we will have defined sequences $\left\langle\mathcal{T}_{\beta}\right\rangle_{\beta<\alpha},\left\langle O_{\beta}, n_{\beta}, \mathcal{Y}_{\beta}, \varsigma_{\beta}\right\rangle_{\beta \leq \alpha}$ with the following properties $(S$ is for stack; see Figure 8):

S1. $O_{0}=M$ and $n_{0}=m$ and $\mathcal{Y}_{0}$ is the trivial tree on $M$ and $\varsigma_{0}: M \rightarrow M$ is the identity.

S2. $n_{\beta} \leq \omega$ and $O_{\beta}$ is a $\mathrm{u}-n_{\beta}$-sound segmented-premouse and if $\beta<\alpha$ then $\mathcal{T}_{\beta}$ is a $\mathrm{u}-n_{\beta}$-maximal tree on $O_{\beta}$ of successor length $<\Omega$.

S3. $O_{\beta+1}=M_{\infty}^{\mathcal{T}_{\beta}}$ and $n_{\beta+1}=\mathrm{u}-\operatorname{deg}^{\mathcal{T}_{\beta}}(\infty)$.

S4. For each limit $\beta \leq \alpha$, there is $\gamma<\beta$ such that for all $\varepsilon \in[\gamma, \beta), b^{\mathcal{T}_{\varepsilon}}$ does not drop in model or degree, $O_{\beta}=M_{\infty}^{\vec{T} ; \beta}$ and $n_{\beta}=\mathrm{u}-\operatorname{deg} \overrightarrow{\vec{T}} \beta \beta(\infty) .{ }^{61}$

S5. $\mathcal{Y}_{\beta}$ is a $\mathrm{u}-m$-maximal tree on $M$, via $\Sigma$, of successor length $<\Omega$, and $\mathrm{u}-\operatorname{deg}^{\mathcal{Y}_{\beta}}(\infty) \geq n_{\beta}$.

S6. $\varsigma_{\beta}: O_{\beta} \rightarrow M_{\infty}^{\mathcal{Y}_{\beta}}$ is a nice $\mathrm{u}-n_{\beta}$-lifting embedding. ${ }^{62}$

S7. For each $\gamma<\beta \leq \alpha, \mathcal{Y}_{\beta}$ is an $\mathcal{Y}_{\gamma}$-terminal inflation of $\mathcal{Y}_{\gamma}$.

S8. For each $\gamma<\beta \leq \alpha, \overrightarrow{\mathcal{T}}\left\lceil[\gamma, \beta)\right.$ drops in model ${ }^{63}$ iff $\mathcal{Y}_{\beta}$ is $\mathcal{Y}_{\gamma}$-terminallymodel-dropping.

S9. For each limit $\beta \leq \alpha$ there is $\varepsilon<\beta$ such that

$$
\forall \delta_{0}, \delta_{1} \text { [if } \varepsilon \leq \delta_{0}<\delta_{1} \leq \beta \text { then } \mathcal{Y}_{\delta_{1}} \text { is } \mathcal{Y}_{\delta_{0}} \text {-terminally-non-dropping]. }
$$

S10. If $\gamma<\beta \leq \alpha$ and $\mathcal{Y}_{\beta}$ is $\mathcal{Y}_{\gamma}$-terminally-non-model-dropping then letting

$$
\pi_{\gamma \beta}: M_{\infty}^{\mathcal{Y}_{\gamma}} \rightarrow M_{\infty}^{\mathcal{Y}_{\beta}}
$$

be $\left(\pi_{\infty}\right)^{\mathcal{Y}_{\gamma} \leadsto \mathcal{Y}_{\beta}}$ (see 4.49), we have $\pi_{\gamma \beta} \circ \varsigma_{\gamma}=\varsigma_{\beta} \circ i^{\vec{T}[\gamma, \beta)}$.

Given these inductive hypotheses, player II plays out round $\alpha$ as follows. We have the nice $\mathrm{u}-n_{\alpha}$-lifting embedding

$$
\varsigma_{\alpha}: O_{\alpha} \rightarrow M_{\infty}^{\mathcal{Y}_{\alpha}}
$$

\footnotetext{
${ }^{61}$ That is, $O_{\beta}$ is the direct limit of the the $O_{\varepsilon}$ for $\varepsilon \in[\gamma, \beta)$, under the iteration maps, and $n_{\beta}=\lim _{\varepsilon \rightarrow \beta} \mathrm{u}-\operatorname{deg}^{\mathcal{T}_{\varepsilon}}(0)$.

${ }^{62}$ In the proof, for $\beta>0, \varsigma_{\beta}$ will be defined as the composition $\varsigma_{\beta}^{1} \circ \varsigma_{\beta}^{0}$.

${ }^{63}$ That is, $b^{\mathcal{T}_{\varepsilon}}$ drops in model for some $\varepsilon \in[\gamma, \beta)$.
} 
and $n_{\alpha} \leq y_{\alpha}=\mathrm{u}-\operatorname{deg}^{\mathcal{Y}_{\alpha}}(\infty)$ and $\operatorname{lh}\left(\mathcal{Y}_{\alpha}\right)<\Omega$. We have the $\left(y_{\alpha}, \Omega+1\right)$-strategy

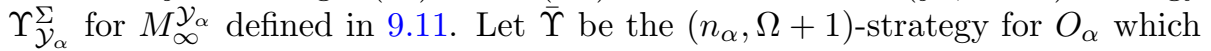
is the $\varsigma_{\alpha}$-pullback of $\Upsilon_{\mathcal{Y}_{\alpha}}^{\Sigma}$. Then player II uses $\bar{\Upsilon}$ to play round $\alpha$ (forming $\mathcal{T}_{\alpha}$ ). So player II does not lose in round $\alpha$.

Now suppose that $\operatorname{lh}\left(\mathcal{T}_{\alpha}\right)<\Omega$, so the game continues. So $O_{\alpha+1}=M_{\infty}^{\mathcal{T}_{\alpha}}$ and $n_{\alpha+1}=\mathrm{u}-\operatorname{deg}^{\mathcal{T}_{\alpha}}(\infty)$. We must define $\mathcal{Y}_{\alpha+1}$ and $\varsigma_{\alpha+1}$ and verify the inductive hypotheses.

Let $\mathcal{U}_{\alpha}=\varsigma_{\alpha} \mathcal{T}_{\alpha}$ be the $\varsigma_{\alpha}$-copy of $\mathcal{T}_{\alpha}$ to a u- $y_{\alpha}$-maximal tree on $M_{\infty}^{\mathcal{Y}_{\alpha}}$. Let $n_{\alpha+1}^{\prime}=\mathrm{u}-\operatorname{deg}^{\mathcal{U}_{\alpha}}(\infty)$. Then $n_{\alpha+1} \leq n_{\alpha+1}^{\prime}$. Let

$$
\varsigma_{\alpha+1}^{0}: O_{\alpha+1} \rightarrow M_{\infty}^{\mathcal{U}_{\alpha}}
$$

be the final copy map, so $\varsigma_{\alpha+1}^{0}$ is nice $\mathrm{u}-n_{\alpha+1}$-lifting.

Now $\mathcal{U}_{\alpha}$ is via $\Upsilon_{\mathcal{Y}_{\alpha}}^{\Sigma}$ and $\ln \left(\mathcal{U}_{\alpha}\right)<\Omega$. Using 9.11, we define

$$
\begin{gathered}
\mathcal{Y}_{\alpha+1}=\mathcal{W}_{\mathcal{Y}_{\alpha}}^{\Sigma}\left(\mathcal{U}_{\alpha}\right), \\
\varsigma_{\alpha+1}^{1}=\varrho_{\mathcal{Y}_{\alpha}}^{\Sigma}\left(\mathcal{U}_{\alpha}\right): M_{\infty}^{\mathcal{U}_{\alpha}} \rightarrow M_{\infty}^{\mathcal{Y}_{\alpha+1}} .
\end{gathered}
$$

So $\varsigma_{\alpha+1}^{1}$ is nice $\mathrm{u}-n_{\alpha+1}^{\prime}$-lifting, $\operatorname{lh}\left(\mathcal{Y}_{\alpha+1}\right)<\Omega$ and $n_{\alpha+1}^{\prime} \leq \mathrm{u}$ - $\operatorname{deg}^{\mathcal{Y}_{\alpha+1}}(\infty)$.

Composing, we define $\varsigma_{\alpha+1}=\varsigma_{\alpha+1}^{1} \circ \varsigma_{\alpha+1}^{0}$, also nice $\mathrm{u}-n_{\alpha+1}$-lifting.

We have verified properties S1-S6 and S9 (some are trivial by induction). It just remains to establish S7, S8 and S10 for $\beta=\alpha+1$.

Suppose first $\gamma=\alpha<\alpha+1=\beta$. Property S7 is directly by $\S 9.1 .1$ (note $\operatorname{lh}\left(\mathcal{U}_{\alpha}\right)<\Omega$ and is a successor). For property $\mathrm{S} 8$, we have that $b^{\mathcal{T}_{\alpha}}$ drops in model iff $b^{\mathcal{U}_{\alpha}}$ drops in model iff (by §9.1.1) $\mathcal{Y}_{\alpha+1}$ is $\mathcal{Y}_{\alpha}$-terminally-modeldropping. And if $\mathcal{Y}_{\alpha+1}$ is $\mathcal{Y}_{\alpha}$-terminally-non-model-dropping, so $b^{\mathcal{T}_{\alpha}}, b^{\mathcal{U}_{\alpha}}$ do not drop in model (but possibly in degree), then again by $\S 9.1 .1$, we have

$$
\pi_{\alpha, \alpha+1}: M_{\infty}^{\mathcal{Y}_{\alpha}} \rightarrow M_{\infty}^{\mathcal{Y}_{\alpha+1}}
$$

(defined in S10), and $\pi_{\alpha, \alpha+1}=\varsigma_{\alpha+1}^{1} \circ i^{\mathcal{U}_{\alpha}}$, so

$$
\pi_{\alpha, \alpha+1} \circ \varsigma_{\alpha}=\varsigma_{\alpha+1} \circ i^{\mathcal{T}_{\alpha}}
$$

as required for property S10.

Finally suppose that $\gamma<\alpha<\alpha+1=\beta$. Properties S7 and S8 follow easily by induction, the facts established above regarding $\mathcal{Y}_{\alpha+1}$, and Lemma 6.2 (commutativity of inflation). For example for property S8: By 6.2, we have that $\mathcal{Y}_{\alpha+1}$ is $\mathcal{Y}_{\gamma}$-terminally-model-dropping iff either $\mathcal{Y}_{\alpha+1}$ is $\mathcal{Y}_{\alpha}$-terminallymodel-dropping or $\mathcal{Y}_{\alpha}$ is $\mathcal{Y}_{\gamma}$-terminally-model-dropping, which by induction and the previous paragraph, suffices. Consider property S10; suppose $\mathcal{Y}_{\alpha+1}$ is $\mathcal{Y}_{\gamma}$-terminally-non-model-dropping. So $b^{\overrightarrow{\mathcal{T}}[\gamma, \alpha+1)}$ does not drop in model, $\mathcal{Y}_{\alpha+1}$ is $\mathcal{Y}_{\alpha}$-terminally-non-model-dropping and $\mathcal{Y}_{\alpha}$ is $\mathcal{Y}_{\gamma}$-terminally-non-modeldropping, and by Lemma $6.2, \pi_{\gamma, \alpha+1}=\pi_{\alpha, \alpha+1} \circ \pi_{\gamma \alpha}$. Property S10 now follows by induction and line (6).

This verifies all the properties at the end of round $\alpha$. 
Now let $\eta<\Omega$ be a limit ordinal, and suppose we have defined

$$
\left\langle\mathcal{T}_{\beta}, O_{\beta}, n_{\beta}, \mathcal{Y}_{\beta}, \varsigma_{\beta}\right\rangle_{\beta<\eta},
$$

and maintained the inductive hypotheses through all $\alpha<\eta$. We need to define $\mathcal{Y}_{\eta}$ and $\varsigma_{\eta}$ and see that the inductive hypotheses hold at $\alpha=\eta$ (of course, $O_{\eta}$ are $n_{\eta}$ will be determined).

We set $\mathcal{Y}_{\eta}$ to be the minimal simultaneous inflation of $\mathscr{T}=\left\{\mathcal{Y}_{\alpha}\right\}_{\alpha<\eta}$ (see Definition 5.1). This exists and $\ln \left(\mathcal{Y}_{\eta}\right)$ is a successor $\xi+1<\Omega$, by Lemma 5.2 and because $\eta<\Omega$ and each $\operatorname{lh}\left(\mathcal{Y}_{\alpha}\right)<\Omega$. Also by Lemma 5.2, there is $\varepsilon<\eta$ such that $\mathcal{Y}_{\eta}$ is $\mathcal{Y}_{\varepsilon}$-terminally-non-dropping; let $\varepsilon_{0}$ be the least such $\varepsilon$. By Lemma 6.2 then, $\mathcal{Y}_{\eta}$ is $\mathcal{Y}_{\delta}$-terminally-non-dropping for all $\delta \in\left[\varepsilon_{0}, \eta\right)$. This gives property $\mathrm{S} 9$. Now let $\varepsilon$ be least such that $\mathcal{Y}_{\eta}$ is $\mathcal{Y}_{\varepsilon}$-terminally-non-modeldropping. Again by Lemma $6.2, \mathcal{Y}_{\eta}$ is $\mathcal{Y}_{\delta}$-terminally-non-model-dropping for each $\delta \in[\varepsilon, \eta)$, and $\mathcal{Y}_{\delta_{1}}$ is $\mathcal{Y}_{\delta_{0}}$-terminally-non-model-dropping for all $\delta_{0}, \delta_{1}$ such that $\varepsilon \leq \delta_{0}<\delta_{1}<\eta$. So by induction and property S8, for all such $\delta_{i}, b^{\mathcal{T}_{\delta_{0}}}$ does not drop in model and $\pi_{\delta_{0} \delta_{1}} \circ \varsigma_{\delta_{0}}=\varsigma_{\delta_{1}} \circ i \vec{T}\left\lceil\left[\delta_{0}, \delta_{1}\right)\right.$. Also, by Lemma 6.2, for all such $\delta_{i}$,

$$
\pi_{\delta_{0} \eta}=\pi_{\delta_{1} \eta} \circ \pi_{\delta_{0} \delta_{1}}: M_{\infty}^{\mathcal{Y}_{\delta_{0}}} \rightarrow M_{\infty}^{\mathcal{Y}_{\eta}} .
$$

Therefore $O_{\eta}=M_{\infty}^{\overrightarrow{\mathcal{T}} \mid \eta}$ and $n_{\eta}=\mathrm{u}-\operatorname{deg} \overrightarrow{\mathcal{T} i \eta}(\infty)$ are well-defined, and we (can and do) define

$$
\varsigma_{\eta}: O_{\eta} \rightarrow M_{\infty}^{\mathcal{Y}_{\eta}}
$$

in the unique manner preserving commutativity, that is,

$$
\varsigma_{\eta} \circ i^{\vec{T}[\delta, \eta)}=\pi_{\delta, \eta} \circ \varsigma_{\delta}
$$

for all $\delta \in[\varepsilon, \eta)$. Then $\varsigma_{\eta}$ is a nice $\mathrm{u}-n_{\eta}$-lifting embedding, and $O_{\eta}$ is wellfounded. It is now easy to verify properties $\mathrm{S} 1-\mathrm{S} 10$.

Finally suppose we have defined $\left\langle\mathcal{T}_{\alpha}\right\rangle_{\alpha<\Omega}$. Then because $\operatorname{cof}(\Omega)>\omega$, we get that for all sufficiently large $\alpha<\Omega, b^{\mathcal{T}_{\alpha}}$ does not drop, and $M_{\infty}^{\overrightarrow{\mathcal{T}}}$ is wellfounded, so player II has won.

This completes the proof of Theorem 9.1.

9.14 Definition. Given $\overrightarrow{\mathcal{T}}=\left\langle\mathcal{T}_{\beta}\right\rangle_{\beta<\alpha}$, etc, satisfying S1-S10, with $\operatorname{lh}(\overrightarrow{\mathcal{T}})=\alpha<$ $\Omega$, we define $\mathcal{W}^{\Sigma}(\overrightarrow{\mathcal{T}})=\mathcal{Y}_{\alpha}$ and $\varsigma^{\Sigma}(\overrightarrow{\mathcal{T}})=\varsigma_{\alpha}: M_{\infty}^{\overrightarrow{\mathcal{T}}}=O_{\alpha} \rightarrow M_{\infty}^{\mathcal{Y}_{\alpha}}$, with notation as above. (We don't try to define these things if $\ln (\overrightarrow{\mathcal{T}})=\Omega$; there seems to be no clear manner in which to define $\mathcal{Y}_{\Omega}$, because $\Sigma$ is not sufficiently powerful.) Given also a tree $\mathcal{T}$ of length $\leq \Omega$, according to the strategy $\bar{\Upsilon}$ for round $\alpha$ defined above, we define $\mathcal{W}^{\Sigma}\left(\overrightarrow{\mathcal{T}}^{\wedge} \mathcal{T}\right)$ to be the corresponding u-m-maximal tree $\mathcal{Y}$ on $M$ (so if $\mathcal{T}$ has successor length and $\mathcal{T}_{\alpha}=\mathcal{T}$ then $\mathcal{Y}_{\alpha+1}=\mathcal{Y}$, and if $\mathcal{T}$ has limit length and $\mathcal{T}_{\alpha}=\mathcal{T}^{\wedge} \bar{\Upsilon}(\mathcal{T})$ then $\mathcal{Y}_{\alpha+1}=\mathcal{Y}^{\wedge} \Sigma(\mathcal{Y})$ ).

9.15 Definition. We write $\Sigma^{\text {st }}$ for the stacks strategy $\Sigma^{*}$ induced by $\Sigma$, defined above. 


\subsubsection{Length $\omega$ stacks of finite trees}

Sketch of Proof of Theorem 9.6. We just consider the fine version. As in Lemma 9.8 we can naturally derive the full strategies from strategies for optimal stacks. So we can restrict our attention to optimal stacks.

Let $M$ be u- $m$-sound and $\Sigma$ be an (u- $m, \Omega+1$ )-strategy for $M$. Then player II wins $\mathcal{G}_{\text {fin,opt }}(M, \mathrm{u}-m, \Omega+1)^{64}$ by using the strategy defined for player II in the iteration game for stacks of length $<\omega$ in the previous proof. Because the normal trees in the stack are finite, there are no branches (of the first tree $\mathcal{T}$ in a stack of length 2) to consider, so no condensation of $\Sigma$ is required to keep the process going. And given a stack $\overrightarrow{\mathcal{T}}=\left\langle\mathcal{T}_{n}\right\rangle_{n<\omega}$, consisting of finite trees, the desired conclusions regarding $\overrightarrow{\mathcal{T}}$ also follow from the limit case of the previous proof, again because we are only inflating finite trees $\mathcal{T}_{n}$. (In a stack $(\mathcal{T}, \mathcal{U})$ of length $2, \mathcal{U}$ could have arbitrary length $\leq \Omega+1$, and also the minimal simultaneous inflation $\mathcal{T}_{\omega}$ of the stack $\left\langle\mathcal{T}_{n}\right\rangle_{n<\omega}$ could seemingly have arbitrary length $<\Omega$, but this is no problem.)

Now suppose that $M$ is MS-indexed, $m$-sound, and $\Sigma$ is an $(m, \Omega+1)$ strategy for $M$. If $M$ is type 3 and $m<\omega$ let $m^{\prime}=m+1$; otherwise let $m^{\prime}=m$. Given a finite $m$-maximal tree $\mathcal{T}$ on $M$, let $\mathcal{T}^{\prime}$ be the corresponding $\mathrm{u}-m^{\prime}$-maximal tree on $M$ (with $\infty$ non- $\mathcal{T}^{\prime}$-special), so $\left(M_{\infty}^{\mathcal{T}^{\prime}}\right)^{\mathrm{pm}}=M_{\infty}^{\mathcal{T}}$. Thus, if $M_{\infty}^{\mathcal{T}}$ is type 3 and $\operatorname{deg}^{\mathcal{T}}(\infty)<\omega$ then $u$ - $\operatorname{deg}^{\mathcal{T}^{\prime}}(\infty)=\operatorname{deg}^{\mathcal{T}}(\infty)+1$; otherwise $\mathrm{u}-\operatorname{deg} \mathcal{T}^{\prime}(\infty)=\operatorname{deg}^{\mathcal{T}}(\infty)$. Moreover, $b^{\mathcal{T}^{\prime}}$ drops iff $b^{\mathcal{T}}$ drops, and if non-dropping then

$$
i^{\mathcal{T}}=i^{\mathcal{T}^{\prime}}\left\lceil M^{\mathrm{sq}}\right.
$$

This generalizes to $m$-maximal finite stacks $\overrightarrow{\mathcal{T}}$ on $M$ consisting of finite trees $\mathcal{T}_{n}$, giving a $\mathrm{u}-m^{\prime}$-maximal finite stack $\overrightarrow{\mathcal{T}}^{\prime}$ with analogous correspondence.

So given such a finite stack $\overrightarrow{\mathcal{T}}$, the u-iteration strategy for $M_{\infty}^{\overrightarrow{\mathcal{T}}^{\prime}}$ given above induces a standard iteration strategy for $M_{\infty}^{\overrightarrow{\mathcal{T}}}$; so player II wins $\mathcal{G}_{\text {fin,opt }}(M, m, \Omega+$ 1). Now let $\overrightarrow{\mathcal{T}}$ have length $\omega$, consisting of finite trees, and $\overrightarrow{\mathcal{T}}^{\prime}$ be its translation to a u- $m^{\prime}$-maximal stack. Let $O_{0}=M$ and $O_{n}=M_{\infty}^{\mathcal{T}_{n-1}}$ for $n>0$; likewise for $O_{n}^{\prime}$. Since for all large $n, b^{\mathcal{T}_{n}^{\prime}}$ does not drop, neither does $b^{\mathcal{T}_{n}}$, and because $i^{\mathcal{T}_{n}}=i^{\mathcal{T}_{n}^{\prime}} \uparrow\left(O_{n}^{\mathrm{sq}}\right)$, we get that $M_{\infty}^{\overrightarrow{\mathcal{T}}}$ is wellfounded. (If $O_{n}$ is non-type 3 for large $n$, this is trivial as $M_{\infty}^{\overrightarrow{\mathcal{T}}^{\prime}}=M_{\infty}^{\overrightarrow{\mathcal{T}}}$. Otherwise, because $\left(O_{n}^{\prime}\right)^{\mathrm{pm}}=O_{n}$ and the iteration maps correspond, we have $\left(M_{\infty}^{\overrightarrow{\mathcal{T}}^{\prime}}\right)^{\mathrm{pm}}=M_{\infty}^{\overrightarrow{\mathcal{T}}}$, which is wellfounded.)

9.16 Remark. We are not sure whether one might extend the preceding theorem to stacks $\left\langle\mathcal{T}_{\alpha}\right\rangle_{\alpha<\lambda}$ of finite trees $\mathcal{T}_{\alpha}$ of arbitrary transfinite length $\lambda$. The method used so far runs into difficulties when $\lambda=\omega+1$, because $\mathcal{Y}_{\omega}$ can be infinite, so that, at least superficially, one seems to need inflation condensation in order to continue. However, the stack $\left\langle\mathcal{T}_{n}\right\rangle_{n<\omega}$ is only a linear stack of finite iterations, so the possible branch choices might be much more limited. Of course in some situations one can just use an absoluteness argument to show that $M_{\infty}^{\overrightarrow{\mathcal{T}}}$

${ }^{64}$ This game is just like $\mathcal{G}_{\text {fin }}$ but player I may not make artificial drops. 
is wellfounded for any $\lambda$ (when each $\mathcal{T}_{\alpha}$ is finite). However particularly when $M$ is active, this is not so easy.

\subsubsection{Variants for partial strategies}

We now state a version of Theorem 9.1 for partial strategies. Typical examples would be a normal strategy $\Sigma$ for $M$ for nice iteration trees (that is, in which all extenders $E=E_{\alpha}^{\mathcal{T}}$ are total, with $\nu_{E}=\varrho(E)$ is inaccessible in $M_{\alpha}^{\mathcal{T}}$ ), or for trees which are based on $M \mid \delta$, where $\delta$ is some $M$-cardinal. In this section we restrict our attention to optimal stacks, but this is only for simplicity, and one can of course consider stacks with artificial drops.

9.17 Definition. Let $M, m, \mathscr{T}$ be as in Definition 4.36 (i) or 4.36(ii) (so in particular, $\mathscr{T}$ is a class of putative trees). Let $D \subseteq \mathscr{T}$ be closed under initial segment. Let $\Omega>\omega$ be regular. We say $\Sigma$ is a $D$ - $(\mathrm{u}-m, \Omega+1)$-strategy iff $\Sigma$ is a function such that $\operatorname{dom}(\Sigma)$ is exactly the set of trees in $D$ of limit length $\leq \Omega$ which are via $\Sigma$, for all $\mathcal{T} \in \operatorname{dom}(\Sigma)$, we have $\mathcal{T}^{\wedge} \Sigma(\mathcal{T}) \in D$, and all putative trees in $D$ via $\Sigma$ have wellfounded well-defined models. We define $D^{*}$-optimal(u- $m, \Omega, \Omega+1)^{*}$-strategy analogously (for some class $D^{*}$ of putative optimal u- $m$-maximal stacks on $M$ ). Likewise for $M, m, \mathscr{T}$ as in 4.36(iii).

Let $\Sigma$ be a conveniently inflationary partial $\mathscr{T}$-strategy for $M$. Suppose $\Sigma$ is a $D$-(u- $m, \Omega+1)$-strategy. Define $D^{\prime}$ and a $D^{\prime}$-optimal-(u- $\left.m, \Omega, \Omega+1\right)^{*}$-strategy $\Sigma^{*}$ for $M$ inductively as follows. Let $\overrightarrow{\mathcal{T}}=\left\langle\mathcal{T}_{\alpha}\right\rangle_{\alpha<\lambda}$ be an optimal stack on $M$, where $\lambda<\Omega$. Say $\overrightarrow{\mathcal{T}}$ is weakly $(\Sigma, D)$-good iff there is a tree $\mathcal{Y}=\mathcal{W}^{\Sigma}(\overrightarrow{\mathcal{T}})$, defined as in Definition 9.14 (so as in the proof of Theorem 9.1), with $\mathcal{Y} \uparrow(\Omega+1)$ in $D$ and via $\Sigma$ (recall that if $\lambda=\alpha+1$ and $\ln \left(\mathcal{T}_{\alpha}\right)=\Omega+1$, we can have $\operatorname{lh}(\mathcal{Y})>\Omega+1)$. We say that $\overrightarrow{\mathcal{T}}$ is $(\Sigma, D)$-good iff $\overrightarrow{\mathcal{T}}$ is weakly $(\Sigma, D)$-good and

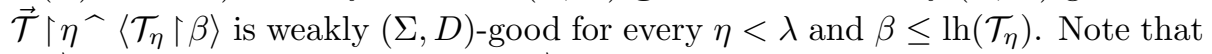
if $\overrightarrow{\mathcal{T}}$ is $(\Sigma, D)$-good then $\mathcal{Y}=\mathcal{W}^{\Sigma}(\overrightarrow{\mathcal{T}})$ is uniquely determined just as before, and $M_{\infty}^{\overrightarrow{\mathcal{T}}}$ exists and is wellfounded and embedded into $M_{\infty}^{\mathcal{Y}}$.

Now define $\Sigma^{*}$ as the partial strategy such that $\overrightarrow{\mathcal{T}} \wedge \mathcal{U} \in \operatorname{dom}\left(\Sigma^{*}\right)$ (where $\overrightarrow{\mathcal{T}}$ is a stack of normal trees and $\mathcal{U}$ is normal) iff there is $b$ such that $\overrightarrow{\mathcal{T}}^{\wedge}\left(\mathcal{U}^{\wedge} b\right)$ is $(\Sigma, D)$-good; in this case set $\Sigma^{*}\left(\overrightarrow{\mathcal{T}}^{\wedge} \mathcal{U}\right)=b$. (And define $D^{\prime}=\operatorname{dom}\left(\Sigma^{*}\right)$.)

Now suppose instead that $M, m, \mathscr{T}$ are as in Definition 4.36(iii), and let $C \subseteq \mathscr{T}$ be closed under initial segment. Let $\Gamma$ be an inconveniently inflationary partial $\mathscr{T}$-strategy, and suppose $\Gamma$ is a $C-(m, \Omega+1)$-strategy. Let $\Sigma$ be the partial $\mathrm{u}-\mathrm{m}^{\prime}$-maximal strategy corresponding to $\Gamma$ (as in Remark $2.16^{65}$ ), and $D$ the class of initial segments unravellings of trees via $\Sigma$ (note these unravellings exist), so $\Sigma$ is a $D$-(u- $m, \Omega+1)$-strategy. Note that all successor length trees via $\Sigma$ can be arbitrarily finitely extended (with $\mathrm{u}-\mathrm{m}^{\prime}$-maximal extensions), because trees via $\Gamma$ can be finitely extended and by Lemma 2.12 . Let $\Sigma^{*}$ be as above. Then we extend $\Gamma$ to the partial optimal stacks strategy $\Gamma^{*}$, which is just the

\footnotetext{
${ }^{65}$ Because $\Gamma$ is a partial $\mathscr{T}$-strategy, all trees via $\Gamma$ are $M$-u-wellfounded by Footnote 31, so Remark 2.16 applies.
} 
partial u-strategy determined by $\Sigma^{*} .66$

Now for example we have:

9.18 Theorem. Let $\Omega>\omega$ be regular. Let $\Sigma$ be an inflationary ${ }^{67}$ partial strategy for $M$. Suppose either

(i) $M \models$ ZFC and $D$ is the the class of normal nice putative trees on $M$, or

(ii) $\Sigma$ is convenient (resp., inconvenient) and there is some $M$-cardinal $\delta<\rho_{0}^{M}$ such that $D$ is the class of $\mathrm{u}$-m-maximal (resp., m-maximal) putative trees on $M$ which are based on $M \mid \delta$.

Suppose $\Sigma$ is a $D$-(u- $m, \Omega+1)$-strategy (resp., $D$ - $(m, \Omega+1)$ ).

Then $\Sigma^{*}$ is a $D^{*}$-optimal-(u-m, $\left.\Omega, \Omega+1\right)^{*}$-strategy (resp., $\left.(m, \Omega, \Omega+1)^{*}\right)$ for $M$, where either:

(i) ' $D^{*}$ is the class of optimal putative stacks of normal nice (putative) trees on $M$, or

(ii)' $D^{*}$ is the class of optimal $\mathrm{u}$-m-maximal (resp., m-maximal) putative stacks on $M$ which are based on $M \mid \delta$,

according to whether (i) or (ii) above holds.

Proof. This is a corollary to the proof of Theorem 9.1. One simply notes that $\mathcal{Y}=\mathcal{W}^{\Sigma}(\overrightarrow{\mathcal{T}})$ is in $D$ (for the relevant $\overrightarrow{\mathcal{T}}$ ). In the nice tree case, this is because all extenders used are copied from some extender used in some $\overrightarrow{\mathcal{T}}$, which is therefore nice in the model it is taken from (and note that all trees are nowhere dropping in this case). In the other case, it uses such copying, and also the commutativity and correspondence of drops described in the proof.

\section{Properties of $\Sigma^{\text {st }}$ and (weak) Dodd-Jensen}

In this final section we show that if $\Sigma$ has certain extra properties, then the stacks strategy $\Sigma^{\text {st }}$ inherits certain extra properties itself. We then give a couple of applications of the theorems to absoluteness of iterability and constructing normal strategies with weak Dodd-Jensen without DC.

\subsection{Normal pullback consistency for $\Sigma^{\text {st }}$}

We record some notation for some standard notions:

10.1 Definition. Let $\Omega>\omega$ be regular. Let $\Gamma$ be a $(\mathrm{u}-m, \Omega, \Omega+1)^{*}$-strategy, with first round $\Gamma^{\mathrm{nm}}$ (nm for normal), so $\Gamma^{\mathrm{nm}}$ is a $(\mathrm{u}-m, \Omega+1)$-strategy). Let $\overrightarrow{\mathcal{T}}$ be a stack via $\Gamma$ of length $<\Omega$, with each component normal tree of length

\footnotetext{
${ }^{66}$ All stacks $\overrightarrow{\mathcal{T}}=\left\langle\mathcal{T}_{\alpha}\right\rangle_{\alpha<\lambda}$ via $\Sigma^{*}$ such that $\mathcal{T}_{\alpha}$ is unravelled for all $\alpha+1<\lambda$, are everywhere unravelable, since all successor length trees via $\Sigma$ are arbitrarily finitely extendible.

${ }^{67}$ Recall this allows both conveniently and inconveniently inflationary.
} 
$<\Omega$. Let $N=M_{\infty}^{\overrightarrow{\mathcal{T}}}$ and $n=\mathrm{u}-\operatorname{deg}^{\overrightarrow{\mathcal{T}}}(\infty)$. Then $\Gamma_{\overrightarrow{\mathcal{T}}}^{\mathrm{nm}}$ denotes the induced $(\mathrm{u}-n, \Omega+1)$-strategy for $N$; that is, $\Gamma_{\overrightarrow{\mathcal{T}}}^{\mathrm{nm}}(\mathcal{U})=\Gamma(\overrightarrow{\mathcal{T}} \wedge \mathcal{U})$ for u-n-maximal trees $\mathcal{U}$ of length $\leq \Omega$. If $b^{\overrightarrow{\mathcal{T}}}$ does not drop in model or degree then $\Gamma_{\leftarrow \overrightarrow{\mathcal{T}}}^{\mathrm{nm}}$ denotes the $i^{\overrightarrow{\mathcal{T}}}$-pullback of $\Gamma_{\overrightarrow{\mathcal{T}}}^{\mathrm{nm}}$, a $(\mathrm{u}-m, \Omega+1)$-strategy for $M$. We say that $\Gamma$ is normally pullback consistent iff for all such $\overrightarrow{\mathcal{T}}$, if $b^{\overrightarrow{\mathcal{T}}}$ does not drop in model or degree then $\Gamma_{\leftarrow \overrightarrow{\mathcal{T}}}^{\mathrm{nm}}=\Gamma^{\mathrm{nm}}$.

10.2 Remark. Given sufficient condensation properties of $\Sigma$, the author expects that one should be able to deduce good condensation properties of $\Sigma^{\text {st }}$, such as pullback consistency (not just normal pullback consistency). In the proof to follow, of the fact that $\Sigma^{\text {st }}$ is normally pullback consistent, assuming sufficient condensation for $\Sigma$, we consider a normal tree $\mathcal{T}$ via $\Sigma$, such that $b^{\mathcal{T}}$ does not drop in model or degree, and letting $N=M_{\infty}^{\mathcal{T}}$, we lift a normal tree $\overline{\mathcal{U}}$ on $M$ to $\mathcal{U}=i^{\mathcal{T}} \overline{\mathcal{U}}$ on $N$, with $\mathcal{U}$ according to $\left(\Sigma^{\text {st }}\right)_{\mathcal{T}}^{\text {nm }}$. Naïvely, one would like to exhibit a tree embedding $\Pi$ from $\overline{\mathcal{U}}$ into $\mathcal{X}=\mathcal{W}_{\mathcal{T}}^{\Sigma}(\mathcal{U})$. The natural naïve candidate for $\Pi$ would be that with $\gamma_{\alpha}=\lambda^{\alpha}$ and $\delta_{\alpha}=\zeta^{\alpha}$, where $\left\langle\lambda^{\alpha}, \zeta^{\alpha}\right\rangle$ arise from the inflation $\mathcal{T} \rightsquigarrow \mathcal{X}$. It is easy to see that this $\Pi$ can fail to be a bounding tree embedding, so inflation condensation does not seem to suffice. In fact, it can fail to be a tree embedding at all, because the requirement that $\gamma_{\alpha} \leq^{\mathcal{X}} \delta_{\alpha}$ can fail. But this can only fail in a special manner, and by slightly generalizing the definition of tree embedding, and demanding condensation of $\Sigma$ with respect to this more general notion, our proof goes through. We now describe the generalization. In the end it is actually more convenient to generalize the demands of normality for the larger tree $\mathcal{X}$, and retain the demand that $\gamma_{\alpha} \leq{ }^{\mathcal{X}} \delta_{\alpha}$, so this is how we proceed.

10.3 Definition. Let $\mathcal{X}$ be an iteration tree on a seg-pm $M$. We say that $\mathcal{X}$ is essentially $\mathrm{u}-m$-maximal iff $\mathcal{X}$ satisfies the requirements of $\mathrm{u}-m$-maximality except that we replace the requirement

$$
\operatorname{ind}\left(E_{\alpha}^{\mathcal{X}}\right) \leq \operatorname{ind}\left(E_{\beta}^{\mathcal{X}}\right) \text { for all } \alpha+1<\beta+1<\ln (\mathcal{X})
$$

with the requirement that for all $\alpha+1<\beta+1<\operatorname{lh}(\mathcal{X})$, either

$-\operatorname{ind}\left(E_{\alpha}^{\mathcal{X}}\right) \leq \operatorname{ind}\left(E_{\beta}^{\mathcal{X}}\right)$, or

$-E_{\alpha}^{\mathcal{X}}$ is of superstrong type and $\lambda\left(E_{\alpha}^{\mathcal{X}}\right)<\operatorname{ind}\left(E_{\beta}^{\mathcal{X}}\right)$.

Recall from $\S 1.1$ that if $M$ is $\lambda$-indexed then every extender in $\mathbb{E}_{+}(M)$ has superstrong type, so in this case, we just require in general that $\lambda\left(E_{\alpha}^{\mathcal{X}}\right)<$ $\operatorname{ind}\left(E_{\beta}^{\mathcal{X}}\right)$.

10.4 Remark. It is easy to see that a $\mathrm{u}-m$-maximal strategy $\Sigma$ yields a corresponding essentially $\mathrm{u}-m$-maximal strategy $\Sigma_{\text {ess }}$; trees $\mathcal{X}$ via $\Sigma_{\text {ess }}$ are those for which there is $\mathcal{X}^{\prime}$ via $\Sigma$ which uses exactly those extenders $E$ such that $E=E_{\alpha}^{\mathcal{X}}$ for some $\alpha$ such that $\operatorname{ind}\left(E_{\alpha}^{\mathcal{X}}\right) \leq \operatorname{ind}\left(E_{\beta}^{\mathcal{X}}\right)$ for all $\beta>\alpha$, and which has corresponding branches. 
10.5 Definition. Let $\mathcal{T}$ be $\mathrm{u}-m$-maximal and $\mathcal{X}$ be essentially $\mathrm{u}-m$-maximal. ${ }^{68}$ An essential tree embedding $\Pi: \mathcal{T} \hookrightarrow_{\text {ess }} \mathcal{X}$ is a system $\Pi=\left\langle I_{\alpha}\right\rangle_{\alpha<\operatorname{lh}(\mathcal{T})}$ satisfying the requirements of a tree embedding, and with corresponding notation, such that whenever $\xi<\eta$ but $\operatorname{ind}\left(E_{\xi}^{\mathcal{X}}\right)>\operatorname{ind}\left(E_{\eta}^{\mathcal{X}}\right)$, then:

$-\eta=\xi+1$, and

- there is $\alpha+1<\operatorname{lh}(\mathcal{T})$ such that $\gamma_{\alpha}<\delta_{\alpha}=\xi+1$ (so $E_{\xi+1}^{\mathcal{X}}=E_{\delta_{\alpha}}^{\mathcal{X}}$ is copied from $\mathcal{T}$ ).

We say that a u- $m$-maximal iteration strategy $\Sigma$ has plus-strong hull condensation iff whenever $\mathcal{X}$ is via $\Sigma_{\text {ess }}$ and $\Pi: \mathcal{T} \hookrightarrow_{\text {ess }} \mathcal{X}$, then $\mathcal{T}$ is via $\Sigma . \quad \dashv$

10.6 Remark. We pause to give a simple example of an essential tree embedding which is not a tree embedding, and which gives a fairly general illustration of how these arise in the proof.

Let $M \models$ ZFC be a mouse. Let $E \in \mathbb{E}^{M}$ and $\mu, \kappa$ be such that

$$
\operatorname{cr}(E)<\mu<\kappa<\nu_{E}
$$

and $\nu_{E}$ is an $M$-cardinal, and letting $U=\operatorname{Ult}(M, E)$, such that there is $F \in \mathbb{E}^{U}$ with ind $(E)<\operatorname{ind}(F)$ and $\operatorname{cr}(F)=\kappa$ and $F$ has superstrong type. Suppose also that $\mu$ is $M$-measurable and there is $G \in \mathbb{E}^{M}$ with $\kappa<\operatorname{ind}(G)<\left(\kappa^{+}\right)^{M}$.

Let $\mathcal{T}$ be the normal tree using $E_{0}^{\mathcal{T}}=E$ and $E_{1}^{\mathcal{T}}=F$, so $0=\operatorname{pred}^{\mathcal{T}}(2)$ and $N={ }_{\text {def }} M_{2}^{\mathcal{T}}=\operatorname{Ult}(M, F)$.

Now let $D \in \mathbb{E}^{M}$ be a normal measure with $\operatorname{cr}(D)=\mu$, so $\operatorname{ind}(D)<\kappa$. Let $\overline{\mathcal{U}}$ be the tree on $M$ using $E_{0}^{\overline{\mathcal{U}}}=D$ and $E_{1}^{\overline{\mathcal{U}}}=i_{D}^{M}(G)$.

Let $\mathcal{U}=i^{\mathcal{T}} \overline{\mathcal{U}}$. So $\mathcal{U}$ is the tree on $N$ using $E_{0}^{\mathcal{U}}=D\left(\operatorname{as~} \operatorname{cr}\left(i^{\mathcal{T}}\right)=\kappa>\mu\right)$ and $E_{1}^{\mathcal{U}}=i_{D}^{N}\left(i^{\mathcal{T}}(G)\right)$. Write $N_{\alpha}=M_{\alpha}^{\mathcal{U}}$.

Now let $\mathcal{X}=\mathcal{W}_{\mathcal{T}}(\mathcal{U})$. Write $\left\langle\lambda^{\alpha}, \zeta^{\alpha}\right\rangle$ for those ordinals arising from the inflation $\mathcal{T} \rightsquigarrow \mathcal{X}$. We have $\mathcal{X}^{0}=\mathcal{T}$, with $N=M_{\infty}^{\mathcal{X}^{0}}$, and $\tau_{0}: N \rightarrow M_{\infty}^{\mathcal{X}^{0}}$ is $\tau_{0}=$ id. Since $\operatorname{ind}(D)<\operatorname{ind}\left(E_{0}^{\mathcal{T}}\right)$, we have $\lambda^{0}=\zeta^{0}=0$ and $E_{\zeta^{0}}^{\mathcal{X}}=D$. So $\lambda^{1}=1$. Then $\mathcal{X}^{1}$ is the tree with $E_{0}^{\mathcal{X}^{1}}=D$, followed by copying $\mathcal{T}=\mathcal{X}^{0}$. So $E_{1}^{\mathcal{X}^{1}}=i_{D}^{M}(E)$, and since $\operatorname{cr}(E)<\mu=\operatorname{cr}(D)$, we have $0=\operatorname{pred}^{\mathcal{X}^{1}}(2)$ (so note that $\left.1=\lambda^{1} \not^{\mathcal{X}^{1}} 2\right)$ and $M_{2}^{\mathcal{X}^{1}}=\operatorname{Ult}\left(M, E_{1}^{\mathcal{X}^{1}}\right)$, and letting

$$
\psi_{1}: U=M_{1}^{\mathcal{X}^{0}} \rightarrow M_{2}^{\mathcal{X}^{1}}
$$

be the copy map, then $E_{2}^{\mathcal{X}^{1}}=\psi_{1}\left(E_{1}^{\mathcal{X}^{0}}\right)=\psi_{1}(F)$. Then since $\kappa=\operatorname{cr}(F)$ and $\operatorname{ind}(D)<\kappa \leq \psi_{1}(\kappa)$ (actually, in this particular example, $\psi_{1}(\kappa)=\kappa$ ), and $\psi_{1}(\kappa)<\nu\left(E_{1}^{\mathcal{X}^{1}}\right)$, therefore $\operatorname{pred}^{\mathcal{X}^{1}}(3)=1=\lambda^{1}$. So $M_{3}^{\mathcal{X}^{1}}=\operatorname{Ult}\left(M_{1}^{\mathcal{X}^{1}}, \psi_{1}(F)\right)$, and $\ln \left(\mathcal{X}^{1}\right)=3+1$, so this completes $\mathcal{X}^{1}$.

We have $\psi_{01}: N=M_{2}^{\mathcal{X}^{0}} \rightarrow M_{3}^{\mathcal{X}^{1}}$ is the final copy map, and $\varrho_{1}: N_{1} \rightarrow M_{3}^{\mathcal{X}^{1}}$ is as defined in the construction of $\Sigma^{\text {st }}$, and $\tau_{1} \circ i_{01}^{\mathcal{U}}=\psi_{01}$. Note that

$$
E_{\zeta^{1}}^{\mathcal{X}}=\varrho_{1}\left(E_{1}^{\mathcal{U}}\right)=\varrho_{1}\left(i_{01}^{\mathcal{U}}\left(i^{\mathcal{T}}(G)\right)\right)=\psi_{01}\left(i^{\mathcal{T}}(G)\right)=i^{\mathcal{X}^{1}}(G)
$$

\footnotetext{
${ }^{68}$ Note that while $\mathcal{X}$ is only essentially $\mathrm{u}-m$-maximal, we still demand that $\mathcal{T}$ be (fully) $\mathrm{u}-m$-maximal.
} 
and so

$$
\lambda\left(E_{2}^{\mathcal{X}^{1}}\right)<\operatorname{ind}\left(E_{\zeta^{1}}^{\mathcal{X}}\right)<\operatorname{ind}\left(E_{2}^{\mathcal{X}^{1}}\right) .
$$

So $\zeta^{1}=2$, so $\lambda^{1} \mathbb{Z}^{\mathcal{X}^{1}} \zeta^{1}$. So if we set $\gamma_{1}=\lambda^{1}$ and $\delta_{1}=\zeta^{1}$, we wouldn't have a tree embedding $\mathcal{\mathcal { U }} \hookrightarrow \mathcal{X}$. However, by replacing $\mathcal{X}$ with the essentially normal tree $\widetilde{\mathcal{X}}$ where $E_{2}^{\widetilde{\mathcal{X}}}=E_{2}^{\mathcal{X}^{1}}$ and then $E_{3}^{\widetilde{\mathcal{X}}}=E_{\zeta^{1}}^{\mathcal{X}}$, we do get an essential tree embedding $\overline{\mathcal{U}} \hookrightarrow_{\text {ess }} \tilde{\mathcal{X}}$.

In the proof below we will actually index the tree $\widetilde{\mathcal{X}}$ differently to this, however. In the situation above we would include two indices $\left(\zeta^{1}, 0\right)$ and $\zeta^{1}$, with $\left(\zeta^{1}, 0\right)<\zeta^{1}$, and set $E_{\left(\zeta^{1}, 0\right)}^{\widetilde{\mathcal{X}}}=E_{2}^{\mathcal{X}^{1}}$ and $E_{\zeta^{1}}^{\widetilde{\mathcal{X}}}=E_{\zeta^{1}}^{\mathcal{X}}$.

10.7 Theorem. Let $\Sigma, \Omega$ be as in Theorem 9.1 (so $\Sigma$ is regularly $(\Omega+1)$ total). Suppose that $\Sigma$ has plus-strong hull condensation (see 10.5). Then $\Sigma^{\text {st }}$ is normally pullback consistent.

Proof. Note that in the definition of normal pullback consistency, we assume that $b^{\overrightarrow{\mathcal{T}}}$ does not drop in model or degree, and in particular, $\overrightarrow{\mathcal{T}}$ is optimal. Therefore we only need consider optimal stacks in the present proof, and that aspect of the construction of $\Sigma^{\text {st }}$. It also easily suffices to consider the case that $\Sigma$ is a convenient strategy.

Let $\overrightarrow{\mathcal{T}}=\left\langle\mathcal{T}_{\alpha}\right\rangle$ be a stack via $\Sigma^{\text {st }}$, such that $b^{\overrightarrow{\mathcal{T}}}$ exists and does not drop in model or degree, $\ln (\overrightarrow{\mathcal{T}})<\Omega$ and $\operatorname{lh}\left(\mathcal{T}_{\alpha}\right)<\Omega$ for each $\alpha$. Let $\mathcal{X}=\mathcal{W}^{\Sigma}(\overrightarrow{\mathcal{T}})$. Then $b^{\mathcal{X}}$ exists and does not drop in model or degree, $\operatorname{lh}(\mathcal{X})<\Omega$, and $i^{\mathcal{X}}=\varsigma \circ i^{\overrightarrow{\mathcal{T}}}$ where $\varsigma=\varsigma^{\Sigma}(\overrightarrow{\mathcal{T}})$; recall $\varsigma: M_{\infty}^{\overrightarrow{\mathcal{T}}} \rightarrow M_{\infty}^{\mathcal{X}}$ (Definition 9.14). Now $\Sigma_{\overrightarrow{\mathcal{T}}}^{\text {nm }}$ is the $\varsigma$-pullback of $\Upsilon_{\mathcal{X}}^{\Sigma}$ (Definition 9.11). Since $i^{\mathcal{X}}=\varsigma \circ i^{\overrightarrow{\mathcal{T}}}$, we may assume that $\overrightarrow{\mathcal{T}}$ consists of a single normal tree $\mathcal{T}$ (so then $\mathcal{X}=\mathcal{T}$ ).

So let $\mathcal{T}$, via $\Sigma$, have length $<\Omega$, and such that $b^{\mathcal{T}}$ does not drop in model or degree. Let $\overline{\mathcal{U}}$ be a $\mathrm{u}$ - $m$-maximal tree on $M$, via $\Sigma_{\leftarrow \mathcal{T}}^{\mathrm{nm}}$. Let $\mathcal{U}=i^{\mathcal{T}} \overline{\mathcal{U}}$, so $\mathcal{T}^{\wedge} \mathcal{U}$ is via $\Sigma^{\text {st }}$. Let $\mathcal{X}=\mathcal{W}_{\mathcal{T}}(\mathcal{U})$. So $<^{\overline{\mathcal{U}}},<^{\mathcal{U}}$ and $<^{\mathcal{X} / \overleftarrow{\mathcal{T}}}$ are identical.

We will define an essentially u- $m$-maximal tree $\widetilde{\mathcal{X}}$ whose corresponding u- $m$ maximal tree is $\mathcal{X}$ (so $\widetilde{\mathcal{X}}$ is via $\Sigma_{\text {ess }}$ ), and exhibit an an essential tree embedding $\Pi: \overline{\mathcal{U}} \hookrightarrow_{\text {ess }} \tilde{\mathcal{X}}$; therefore (by plus-strong hull condensation) $\overline{\mathcal{U}}$ is via $\Sigma$, giving normal pullback consistency for this case.

Let $\iota=\operatorname{lh}(\overline{\mathcal{U}})$ and $\left\langle\lambda^{\eta}, \zeta^{\eta}\right\rangle_{\eta<\iota}$ be determined by the inflation $\mathcal{T} \rightsquigarrow \mathcal{X}$.

For convenience, we index $\widetilde{\mathcal{X}}$ with a set $D$ such that

$$
\operatorname{lh}(\mathcal{X}) \subseteq D \subseteq \ln (\mathcal{X}) \cup(\operatorname{lh}(\mathcal{X}) \times\{0\})
$$

For $(\zeta, 0) \in D$, we set $(\zeta, 0)<\zeta$, and $(\beta, 0)<\beta<(\zeta, 0)$ for all $\beta<\zeta$. We will have $E_{\zeta}^{\widetilde{\mathcal{X}}}=E_{\zeta}^{\mathcal{X}}$ for every $\zeta+1<\ln (\mathcal{X})$. The consecutive pairs $x<x^{\prime} \in D$ such that $\operatorname{ind}\left(E_{x}^{\widetilde{\mathcal{X}}}\right)>\operatorname{ind}\left(E_{x^{\prime}}^{\widetilde{\mathcal{X}}}\right)$ will be exactly those of the form $x=(\zeta, 0), x^{\prime}=\zeta$ where $(\zeta, 0) \in D$.

For each $\zeta<\operatorname{lh}(\mathcal{X})$, we put $(\zeta, 0) \in D$ iff there is $\alpha+1<\operatorname{lh}(\overline{\mathcal{U}})$ such that

$$
\zeta=\zeta^{\alpha} \text { and } \zeta^{\alpha}+1<\ln \left(\mathcal{X}^{\alpha}\right) \text { and } \lambda\left(E_{\zeta^{\alpha}}^{\mathcal{X}^{\alpha}}\right)<\operatorname{ind}\left(E_{\zeta^{\alpha}}^{\mathcal{X}}\right) .
$$


Of course, whenever $\zeta^{\beta}+1<\ln \left(\mathcal{X}^{\beta}\right)$, we have $\operatorname{ind}\left(E_{\zeta^{\beta}}^{\mathcal{X}}\right)<\operatorname{ind}\left(E_{\zeta^{\beta}}^{\mathcal{X}^{\beta}}\right)$. So if $\left(\zeta^{\alpha}, 0\right) \in D$ then $E_{\zeta^{\alpha}}^{\mathcal{X}^{\alpha}}$ has superstrong type (and note that this extender is copied from $\mathcal{T}$, via the inflation $\mathcal{T} \rightsquigarrow \mathcal{X})$. If $\left(\zeta^{\alpha}, 0\right) \in D$ then define $\zeta_{*}^{\alpha}=\zeta^{\alpha}+1$; otherwise define $\zeta_{*}^{\alpha}=\zeta^{\alpha}$.

If $\left(\zeta^{\alpha}, 0\right) \in D$ then we set $E_{\left(\zeta^{\alpha}, 0\right)}^{\tilde{\mathcal{X}}}=E_{\zeta^{\alpha}}^{\mathcal{X}^{\alpha}}$ and $E_{\zeta^{\alpha}}^{\tilde{\mathcal{X}}}=E_{\zeta^{\alpha}}^{\mathcal{X}}$, so $E_{\left(\zeta^{\alpha}, 0\right)}^{\tilde{\mathcal{X}}}$ has superstrong type and

$$
\lambda\left(E_{\left(\zeta^{\alpha}, 0\right)}^{\widetilde{\mathcal{X}}}\right)<\operatorname{ind}\left(E_{\zeta^{\alpha}}^{\widetilde{\mathcal{X}}}\right)<\operatorname{ind}\left(E_{\left(\zeta^{\alpha}, 0\right)}^{\widetilde{\mathcal{X}}}\right)
$$

we will also have here that $\lambda^{\alpha}<^{\mathcal{X}^{\alpha}} \zeta^{\alpha}+1 \in b^{\mathcal{X}^{\alpha}}$ and $\lambda^{\alpha}<^{\widetilde{\mathcal{X}}} \zeta^{\alpha}$.

With this notation, the desired essential tree embedding $\Pi: \overline{\mathcal{U}} \hookrightarrow_{\text {ess }} \widetilde{\mathcal{X}}$ is that with

$$
I_{\alpha}=\left[\gamma_{\alpha}, \delta_{\alpha}\right]_{\tilde{\mathcal{X}}}=\left[\lambda^{\alpha}, \zeta^{\alpha}\right]_{\tilde{\mathcal{X}}}
$$

We will verify that this does indeed work. We write $\pi_{\alpha}: M_{\alpha} \rightarrow M_{\lambda^{\alpha}}^{\tilde{\mathcal{X}}}$ for the associated embedding.

Adopt the notation from the construction of $\mathcal{X}=\mathcal{W}_{\mathcal{T}}^{\Sigma}(\mathcal{U})(\S 9.1 .1)$. So $N_{\eta}=$ $M_{\eta}^{\mathcal{U}}$, etc. Write $M_{\eta}=M_{\eta}^{\overline{\mathcal{U}}}$ and $\bar{E}_{\eta}=E_{\eta}^{\overline{\mathcal{U}}}$ and $E_{\eta}=E_{\eta}^{\mathcal{U}}$ and $F_{\eta}=E_{\zeta^{\eta}}^{\tilde{\mathcal{X}}}$. Let $\varphi_{\eta}: M_{\eta} \rightarrow N_{\eta}$ be the copy map. We have $\varrho_{\eta}: N_{\eta} \rightarrow M_{\infty}^{\mathcal{X}^{\eta}}$. So

$$
F_{\eta}=E_{\zeta^{\eta}}^{\tilde{\mathcal{X}}}=E_{\zeta^{\eta}}^{\mathcal{X}}=\varrho_{\eta}\left(E_{\eta}\right)=\varrho_{\eta}\left(\varphi_{\eta}\left(\bar{E}_{\eta}\right)\right) .
$$

Note that $\mathcal{U}$ and $\overline{\mathcal{U}}$ have matching drop and degree structure (using that copying propagates near embeddings, by the argument of [9]), and when $[0, \eta]_{\overline{\mathcal{U}}}$ drops in model or degree, we have $\lambda^{\eta}+1=\zeta^{\eta}+1=\operatorname{lh}\left(\mathcal{X}^{\eta}\right)$ and $\left(\zeta^{\eta}, 0\right) \notin D$ and $i_{\lambda^{\eta} \zeta^{\eta}}^{\mathcal{X}^{\eta}}=\mathrm{id}=i_{\lambda^{\eta} \zeta^{\eta}}^{\widetilde{\mathcal{X}}}$, and so in this case various things stated below simplify or trivialize.

We will prove the following facts, by induction on $\eta<\operatorname{lh}(\overline{\mathcal{U}})$ :

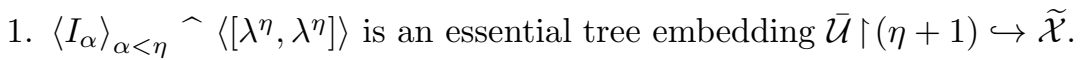

2. If $\alpha<\eta$ then $\lambda^{\alpha} \leq^{\widetilde{\mathcal{X}}} \zeta^{\alpha}$ and $\left(\lambda^{\alpha}, \zeta^{\alpha}\right]_{\widetilde{\mathcal{X}}} \cap \mathscr{D}_{\operatorname{deg}}^{\tilde{\mathcal{X}}}=\emptyset$, so

$$
F_{\alpha}=i_{\lambda^{\alpha} \zeta^{\alpha}}^{\tilde{\mathcal{X}}}\left(\pi_{\alpha}\left(\bar{E}_{\alpha}\right)\right)
$$

by condition 1 . Moreover:

(a) If $\left(\zeta^{\alpha}, 0\right) \notin D$ then $\lambda^{\alpha} \leq{ }^{\mathcal{X}^{\alpha}} \zeta^{\alpha} \in b^{\mathcal{X}^{\alpha}}$ and $\operatorname{cr}\left(i_{\zeta^{\alpha} \infty}^{\mathcal{X}^{\alpha}}\right)>\operatorname{ind}\left(E_{\zeta^{\alpha}}^{\mathcal{X}}\right)$.

(b) If $\left(\zeta^{\alpha}, 0\right) \in D$ then $\lambda^{\alpha}<^{\mathcal{X}^{\alpha}} \zeta^{\alpha}+1 \in b^{\mathcal{X}^{\alpha}}$ and $\operatorname{cr}\left(i_{\zeta^{\alpha}+1, \infty}^{\mathcal{X}^{\alpha}}\right)>\operatorname{ind}\left(E_{\zeta^{\alpha}}^{\mathcal{X}}\right)$ and $\lambda^{\alpha}<\tilde{\mathcal{X}} \zeta^{\alpha}$.

(Recall that $\left(\zeta^{\alpha}, 0\right) \in D$ iff $\left[\zeta^{\alpha}+1<\ln \left(\mathcal{X}^{\alpha}\right)\right.$ and $\left.\lambda\left(E_{\zeta^{\alpha}}^{\mathcal{X}^{\alpha}}\right)<\operatorname{ind}\left(E_{\zeta^{\alpha}}^{\mathcal{X}}\right)\right]$.)

3. $\lambda^{\eta} \in b^{\mathcal{X}^{\eta}}$ and $\left(\lambda^{\eta}, \infty\right] \mathcal{X}^{\eta}$ does not drop in model or degree.

4. $\varrho_{\eta} \circ \varphi_{\eta}=i_{\lambda^{\eta} \infty}^{\mathcal{X}^{\eta}} \circ \pi_{\eta}$ (cf. Figure 9). 


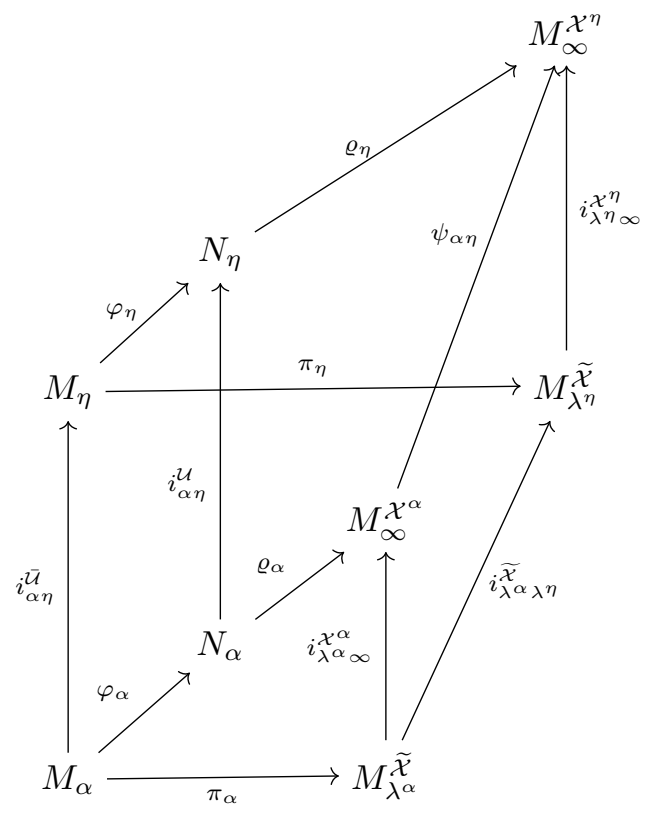

Figure 9: The diagram commutes, where $(\alpha, \eta]_{\mathcal{U}}$ does not drop in model.

5. If $\alpha<^{\overline{\mathcal{U}}} \eta$ and $(\alpha, \eta]_{\overline{\mathcal{U}}}$ does not drop in model then the diagram in Figure 9 commutes.

Note that if $[0, \eta]_{\overline{\mathcal{U}}}$ drops in model or degree then $\lambda^{\eta}+1=\ln \left(\mathcal{X}^{\eta}\right)$ and $M_{\lambda^{\eta}}^{\tilde{\mathcal{X}}}=M_{\lambda^{\eta}}^{\mathcal{X}^{\eta}}$ and $i_{\lambda^{\eta} \infty}^{\mathcal{X}^{\eta}}=$ id, so condition 4 becomes $\varrho_{\eta} \circ \varphi_{\eta}=\pi_{\eta}$, and if $\alpha<^{\overline{\mathcal{U}}} \eta$ and $[0, \alpha]_{\overline{\mathcal{U}}}$ drops in model or degree then the diagram in Figure 9 simplifies to become that in Figure 10.

Consider first the case that $\eta=0$. Conditions 2 and 5 are trivial. The essential tree embedding referred to in condition 1 is just the trivial one. Recall that $\mathcal{T}=\mathcal{X}^{0}$ does not drop in model or degree on $b^{\mathcal{T}}$, and of course $\lambda^{0}=0$. So condition 3 is immediate. And condition 4 holds because $\varphi_{0}=i^{\mathcal{T}}$ and $\varrho_{0}=\mathrm{id}$ and $\pi_{0}=$ id and $i_{0 \infty}^{\mathcal{X}^{0}}=\varphi_{0}$.

For limit $\eta$, condition 2 is trivial by induction, and the other conditions follow by induction using the commutativity of the various maps discussed in $\S 8$ and the construction of $\Sigma^{\text {st }}$. We leave the details to the reader.

So consider the case that $\eta=\beta+1$.

Condition 2: Consider the case that $\alpha=\beta$. Since $\mathcal{U}=i^{\mathcal{T}} \overline{\mathcal{U}}=\varphi_{0} \overline{\mathcal{U}}$ and by induction (conditions 3 and 4$), \lambda^{\beta} \in b^{\mathcal{X}^{\beta}}$ and $\left(\lambda^{\beta}, \infty\right]_{\mathcal{X}^{\beta}} \cap \mathscr{D}_{\operatorname{deg}}^{\mathcal{X}^{\beta}}=\emptyset$ and

$$
E_{\zeta^{\beta}}^{\mathcal{X}}=\varrho_{\beta}\left(\varphi_{\beta}\left(E_{\beta}^{\overline{\mathcal{U}}}\right)\right)=i_{\lambda^{\beta} \infty}^{\mathcal{X}^{\beta}}\left(\pi_{\beta}\left(E_{\beta}^{\overline{\mathcal{U}}}\right)\right),
$$




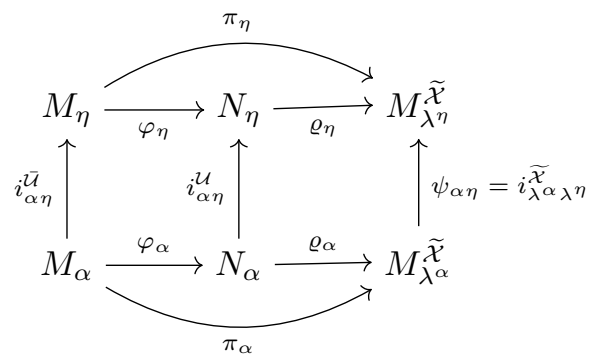

Figure 10: The simplification of Figure 9 when $[0, \alpha]_{\mathcal{\mathcal { U }}}$ drops in model or degree.

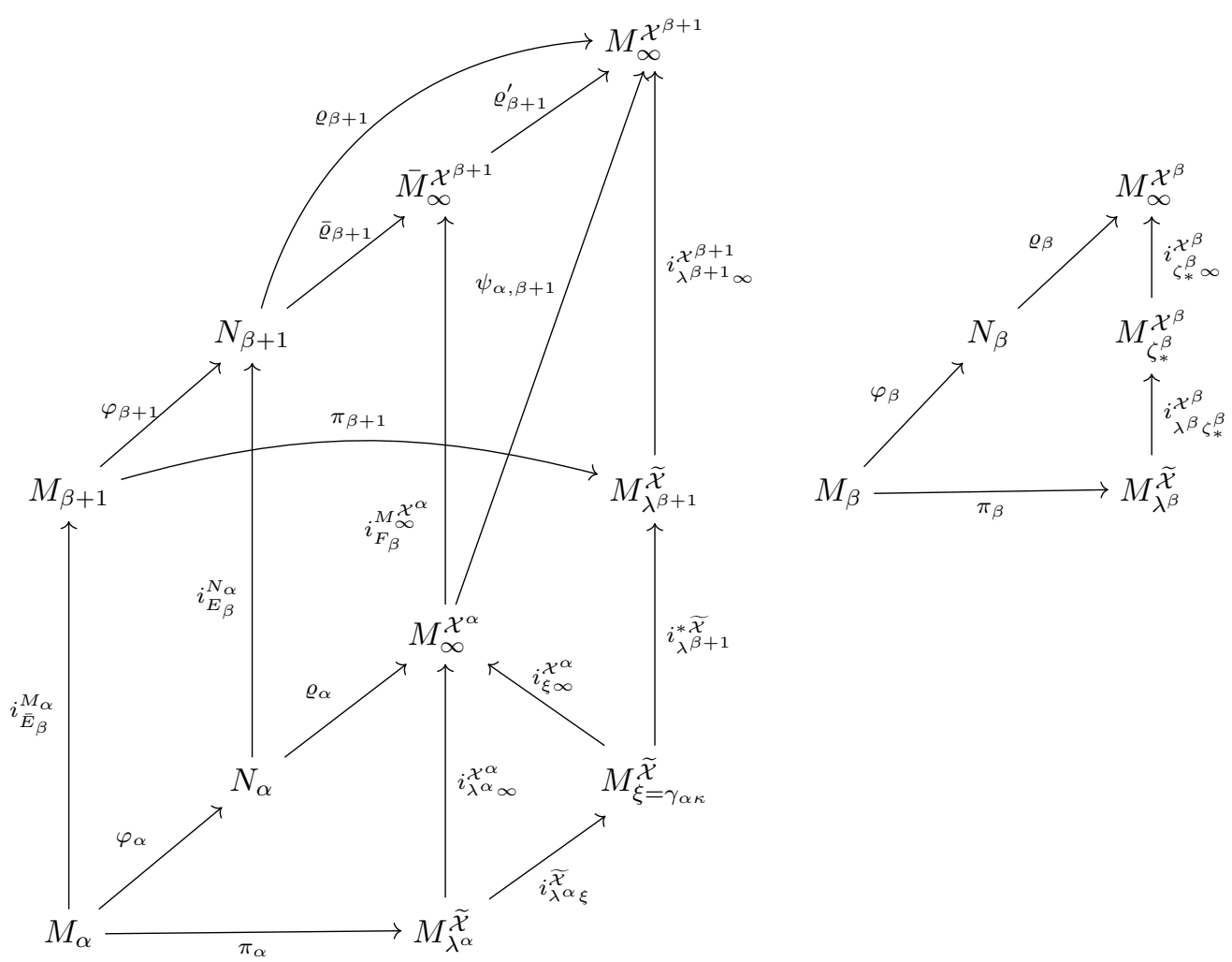

Figure 11: The diagrams commute (here $\left.\beta+1 \notin \mathscr{D}^{\overline{\mathcal{U}}}\right)$. We have $\alpha=\operatorname{pred}^{\overline{\mathcal{U}}}(\beta+1)$ and $\xi=\gamma_{\alpha \kappa}=\gamma_{\Pi \alpha \kappa}$, where $\kappa=\operatorname{cr}\left(\bar{E}_{\beta}\right)$ and $\Pi$ is the essential tree embedding under construction. Note that $M_{\zeta_{*}^{\beta}}^{\mathcal{X}^{\beta}}=M_{\zeta^{\beta}}^{\widetilde{\mathcal{X}}}$ and $i_{\lambda^{\beta} \zeta_{*}^{\beta}}^{\mathcal{X}^{\beta}}=i_{\lambda^{\beta} \zeta^{\beta}}^{\widetilde{\mathcal{X}}}$. 
so $E_{\zeta^{\beta}}^{\mathcal{X}} \in \operatorname{rg}\left(i_{\lambda^{\beta} \infty}^{\mathcal{X}^{\beta}}\right)$. And $\zeta^{\beta}$ is the least $\zeta$ such that $E_{\zeta^{\beta}}^{\mathcal{X}} \in \mathbb{E}_{+}\left(M_{\zeta}^{\mathcal{X}^{\beta}}\right)$. So ${ }^{69}$

$$
\operatorname{ind}\left(E_{\xi}^{\mathcal{X}^{\beta}}\right) \leq \operatorname{ind}\left(E_{\zeta^{\beta}}^{\mathcal{X}}\right)<\operatorname{ind}\left(E_{\xi^{\prime}}^{\mathcal{X}^{\beta}}\right)
$$

for all $\xi<\zeta^{\beta}$ and $\xi^{\prime} \geq \zeta^{\beta}$ with $\xi^{\prime}+1<\operatorname{lh}\left(\mathcal{X}^{\beta}\right)$.

Now suppose that $\left(\zeta^{\beta}, 0\right) \notin D$; we must verify condition 2 a. So if $\zeta^{\beta}+1<$ $\ln \left(\mathcal{X}^{\beta}\right)$ then $\operatorname{ind}\left(E_{\zeta^{\beta}}^{\mathcal{X}}\right)<\lambda\left(E_{\zeta^{\beta}}^{\mathcal{X}^{\beta}}\right)$. We may easily assume that $\zeta^{\beta}+1<\operatorname{lh}\left(\mathcal{X}^{\beta}\right)$. Suppose $\zeta^{\beta} \notin b^{\mathcal{X}^{\beta}}$, and let $\zeta \geq \zeta^{\beta}$ be least such that $\zeta+1 \in b^{\mathcal{X}^{\beta}}$. Then

$$
\lambda^{\beta} \leq \mathcal{X}^{\mathcal{X}^{\beta}} \xi=\operatorname{def}_{\operatorname{pred}^{\mathcal{X}^{\beta}}}(\zeta+1)<\zeta^{\beta}<\zeta+1,
$$

so

$$
\operatorname{cr}\left(E_{\zeta}^{\mathcal{X}^{\beta}}\right)<\widetilde{\nu}\left(E_{\xi}^{\mathcal{X}^{\beta}}\right)<\lambda\left(E_{\zeta^{\beta}}^{\mathcal{X}^{\beta}}\right) \leq \lambda\left(E_{\zeta}^{\mathcal{X}^{\beta}}\right),
$$

and as $E_{\zeta^{\beta}}^{\mathcal{X}} \in \operatorname{rg}\left(i_{\lambda^{\beta} \infty}^{\mathcal{X}^{\beta}}\right)$ and $\operatorname{ind}\left(E_{\zeta^{\beta}}^{\mathcal{X}}\right)<\lambda\left(E_{\zeta^{\beta}}^{\mathcal{X}^{\beta}}\right)$, therefore $\operatorname{ind}\left(E_{\zeta^{\beta}}^{\mathcal{X}}\right)<\operatorname{cr}\left(E_{\zeta}^{\mathcal{X}^{\beta}}\right)$. But then $E_{\zeta^{\beta}}^{\mathcal{X}} \in \mathbb{E}\left(M_{\xi}^{\mathcal{X}^{\beta}}\right)$, contradicting the minimality of $\zeta^{\beta}$. So $\lambda^{\beta} \leq^{\mathcal{X}^{\beta}} \zeta^{\beta} \in$ $b^{\mathcal{X}^{\beta}}$. Let $\zeta+1=\operatorname{succ}^{\mathcal{X}^{\beta}}\left(\zeta^{\beta}, \infty\right)$. If

$$
\operatorname{cr}\left(E_{\zeta}^{\mathcal{X}^{\beta}}\right)=\operatorname{cr}\left(i_{\zeta^{\beta} \infty}^{\mathcal{X}^{\beta}}\right)<\operatorname{ind}\left(E_{\zeta^{\beta}}^{\mathcal{X}}\right)
$$

then

$$
\operatorname{cr}\left(E_{\zeta}^{\mathcal{X}^{\beta}}\right)<\operatorname{ind}\left(E_{\zeta^{\beta}}^{\mathcal{X}}\right)<\lambda\left(E_{\zeta^{\beta}}^{\mathcal{X}^{\beta}}\right) \leq \lambda\left(E_{\zeta}^{\mathcal{X}^{\beta}}\right),
$$

again contradicting the fact that $E_{\zeta^{\beta}}^{\mathcal{X}} \in \operatorname{rg}\left(i_{\lambda^{\beta} \infty}^{\mathcal{X}^{\beta}}\right)$. This gives 2a.

Now suppose $\left(\zeta^{\beta}, 0\right) \in D$; we verify 2 b. So $\zeta^{\beta}+1<\ln \left(\mathcal{X}^{\beta}\right)$ and

$$
\lambda\left(E_{\zeta^{\beta}}^{\mathcal{X}^{\beta}}\right)<\operatorname{ind}\left(E_{\zeta^{\beta}}^{\mathcal{X}}\right)<\operatorname{ind}\left(E_{\zeta^{\beta}}^{\mathcal{X}^{\beta}}\right) .
$$

Let $\zeta \geq \zeta^{\beta}$ be least such that $\zeta+1 \in b^{\mathcal{X}^{\beta}}$, and $\xi=\operatorname{pred}^{\mathcal{X}^{\beta}}(\zeta+1)$. Then $\lambda^{\beta} \leq \mathcal{X}^{\beta} \xi \leq \zeta^{\beta}$, so

$$
\operatorname{cr}\left(E_{\zeta}^{\mathcal{X}^{\beta}}\right)<\widetilde{\nu}\left(E_{\xi}^{\mathcal{X}^{\beta}}\right) \leq \lambda\left(E_{\zeta^{\beta}}^{\mathcal{X}^{\beta}}\right) \leq \lambda\left(E_{\zeta}^{\mathcal{X}^{\beta}}\right),
$$

and since $E_{\zeta^{\beta}}^{\mathcal{X}} \in \operatorname{rg}\left(i_{\lambda^{\beta} \infty}^{\mathcal{X}^{\beta}}\right)$ and by line (7), it follows that $\zeta=\zeta^{\beta}$, so $\zeta^{\beta}+1 \in b^{\mathcal{X}^{\beta}}$, so $\lambda^{\beta}<^{\mathcal{X}^{\beta}} \zeta^{\beta}+1$. The fact that $\lambda^{\beta}<^{\tilde{\mathcal{X}}} \zeta^{\beta}$ follows immediately by definition; note that the role of $\zeta^{\beta}$ in $\tilde{\mathcal{X}}$ corresponds to $\zeta^{\beta}+1$ in $\mathcal{X}^{\beta}$, as $E_{\left(\zeta^{\beta}, 0\right)}^{\widetilde{\mathcal{X}}}=E_{\zeta^{\beta}}^{\mathcal{X}^{\beta}}$. Similarly, $\operatorname{ind}\left(E_{\zeta^{\beta}}^{\mathcal{X}}\right)<\operatorname{cr}\left(i_{\zeta^{\beta}+1, \infty}^{\mathcal{X}^{\beta}}\right)$. This gives $2 \mathrm{~b}$.

We can now complete the proof of condition 2. We have:

$-\mathcal{X}^{\beta}\left\lceil\left(\zeta_{*}^{\beta}+1\right)\right.$ is the $\mathrm{u}-m$-maximal tree corresponding to $\tilde{\mathcal{X}} \uparrow\left(\zeta^{\beta}+1\right) .{ }^{70}$

\footnotetext{
${ }^{69}$ An earlier draft of this paper had the first inequality here as strict $<$, but it seems we might need to allow $\leq$. However, this has little bearing on the proof.

${ }^{70}$ Recall that $\widetilde{\mathcal{X}}$ is only essentially u-m-maximal, and note that if $\left(\zeta^{\beta}, 0\right) \in D$, so $\zeta_{*}^{\beta}=\zeta^{\beta}+1$, then both $\tilde{\mathcal{X}} \uparrow\left(\zeta^{\beta}+1\right)$ and $\mathcal{X}^{\beta}\left\lceil\zeta_{*}^{\beta}+1\right.$ use last extender $E_{\left(\zeta^{\beta}, 0\right)}^{\widetilde{\mathcal{X}}}=E_{\zeta^{\beta}}^{\mathcal{X}^{\beta}}$.
} 
$-\lambda^{\beta} \leq^{\mathcal{X}^{\beta}} \zeta_{*}^{\beta} \in b^{\mathcal{X}^{\beta}}$ and $\left(\lambda^{\beta}, \infty\right]_{\mathcal{X}^{\beta}}$ does not drop in model or degree,

- $\left(\lambda^{\beta}, \zeta^{\beta}\right]_{\tilde{\mathcal{X}}}$ does not drop in model or degree,

$-M_{\zeta^{\beta}}^{\tilde{\mathcal{X}}}=M_{\zeta_{*}^{\beta}}^{\mathcal{X}^{\beta}}$ and $i_{\lambda^{\beta} \zeta^{\beta}}^{\tilde{\mathcal{X}}}=i_{\lambda^{\beta} \zeta_{*}^{\beta}}^{\mathcal{X}^{\beta}}$,

$-E_{\zeta^{\beta}}^{\widetilde{\mathcal{X}}}=i_{\lambda^{\beta} \infty}^{\mathcal{X}^{\beta}}\left(\pi_{\beta}\left(E_{\beta}^{\overline{\mathcal{U}}}\right)\right)$ and $\operatorname{ind}\left(E_{\zeta^{\beta}}^{\widetilde{\mathcal{X}}}\right)<\operatorname{cr}\left(i_{\zeta^{\beta} \infty}^{\mathcal{X}^{\beta}}\right)$, so

$-E_{\zeta^{\beta}}^{\tilde{\mathcal{X}}}=i_{\lambda^{\beta} \zeta^{\beta}}^{\widetilde{\mathcal{X}}}\left(\pi_{\beta}\left(E_{\beta}^{\overline{\mathcal{U}}}\right)\right)$.

Condition 1: By induction, $\left\langle I_{\alpha}\right\rangle_{\alpha<\beta} \widehat{\langle}\left\langle\left[\lambda^{\beta}, \lambda^{\beta}\right]\right\rangle$ is an essential tree embed$\operatorname{ding} \overline{\mathcal{U}} \uparrow(\beta+1) \hookrightarrow \tilde{\mathcal{X}}$. But then by condition 2 and as $\lambda^{\beta+1}=\zeta^{\beta}+1$,

$$
\left\langle I_{\alpha}\right\rangle_{\alpha \leq \beta} \hat{\langle}\left\langle\left[\lambda^{\beta+1}, \lambda^{\beta+1}\right]\right\rangle
$$

is also an essential tree embedding $\overline{\mathcal{U}} \uparrow(\beta+2) \hookrightarrow \widetilde{\mathcal{X}}$.

Conditions 3, 4, 5: We consider the case that $[0, \beta+1]_{\overline{\mathcal{U}}} \cap \mathscr{D}_{\text {deg }}^{\overline{\mathcal{A}}}=\emptyset$, and leave the other case to the reader. By induction, it suffices to verify condition 3 for $\eta=\beta+1$ and to verify that the diagram on the left of Figure 11 commutes, for the current $\beta$ and $\alpha=\operatorname{pred}^{\bar{u}}(\beta+1)$ (by induction and condition 2, the diagram on the right of Figure 11 commutes). Note the embeddings $i_{\bar{E}_{\beta}}^{M_{\alpha}}, i_{E_{\beta}}^{N_{\alpha}}$ and $i_{F_{\beta}}^{M_{\infty}^{\mathcal{X}^{\alpha}}}$ are the ultrapower embeddings associated to $\operatorname{Ult}_{\mathrm{u}-m}\left(M_{\alpha}, \bar{E}_{\beta}\right)$, etc.

As in the figure, let $\kappa=\operatorname{cr}\left(\bar{E}_{\beta}\right)$ and $\xi=\gamma_{\alpha \kappa}=\gamma_{\Pi \alpha \kappa}$, so $\xi=\operatorname{pred}^{\tilde{\mathcal{X}}}\left(\lambda^{\beta+1}\right)$ and $\xi \in I_{\alpha}=\left[\lambda^{\alpha}, \zeta^{\alpha}\right]_{\tilde{\mathcal{X}}}$. Let

$$
\mu=i_{\lambda^{\alpha} \xi}^{\tilde{\mathcal{X}}}\left(\pi_{\alpha}(\kappa)\right)=i_{\lambda^{\alpha} \zeta^{\alpha}}^{\widetilde{\mathcal{X}}}\left(\pi_{\alpha}(\kappa)\right) .
$$

Then $\xi \in\left[\lambda^{\alpha}, \zeta_{*}^{\alpha}\right] \mathcal{X}^{\alpha}$ and

$$
\mu=i_{\lambda^{\alpha} \xi}^{\mathcal{X}^{\alpha}}\left(\pi_{\alpha}(\kappa)\right)=i_{\lambda^{\alpha} \infty}^{\mathcal{X}^{\alpha}}\left(\pi_{\alpha}(\kappa)\right)<\operatorname{cr}\left(i_{\xi \infty}^{\mathcal{X}^{\alpha}}\right)
$$

and either:

- $\left(\zeta^{\alpha}, 0\right) \notin D$ and $\left[\lambda^{\alpha}, \zeta^{\alpha}\right]_{\widetilde{\mathcal{X}}}=\left[\lambda^{\alpha}, \zeta_{*}^{\alpha}\right]_{\mathcal{X}^{\alpha}}$, or

- $\left(\zeta^{\alpha}, 0\right) \in D$ and $\xi \in\left[\lambda^{\alpha}, \varepsilon\right]_{\tilde{\mathcal{X}}}=\left[\lambda^{\alpha}, \varepsilon\right]_{\mathcal{X}^{\alpha}}$ where

$$
\varepsilon=\operatorname{pred}^{\mathcal{X}^{\alpha}}\left(\zeta^{\alpha}+1\right)=\operatorname{pred}^{\tilde{\mathcal{X}}}\left(\zeta^{\alpha}\right) .
$$

For suppose $\left(\zeta^{\alpha}, 0\right) \notin D$. Then

$$
\zeta^{\alpha}=\zeta_{*}^{\alpha} \text { and } \xi \in\left[\lambda^{\alpha}, \zeta^{\alpha}\right]_{\tilde{\mathcal{X}}}=\left[\lambda^{\alpha}, \zeta_{*}^{\alpha}\right]_{\mathcal{X}^{\alpha}} \text { and } i_{\lambda^{\alpha} \zeta^{\alpha}}^{\widetilde{\mathcal{X}}}=i_{\lambda^{\alpha} \zeta^{\alpha}}^{\mathcal{X}^{\alpha}}
$$

Also $\kappa<\widetilde{\nu}\left(E_{\alpha}^{\overline{\mathcal{U}}}\right)$, so $\mu<\widetilde{\nu}\left(E_{\zeta^{\alpha}}^{\widetilde{\mathcal{X}}}\right)<\operatorname{ind}\left(E_{\zeta^{\alpha}}^{\widetilde{\mathcal{X}}}\right)<\operatorname{cr}\left(i_{\zeta^{\alpha} \infty}^{\mathcal{X}^{\alpha}}\right)$, which easily suffices. 
Now suppose instead that $\left(\zeta^{\alpha}, 0\right) \in D$. Then $M_{\zeta^{\alpha}}^{\widetilde{\mathcal{X}}} \| \operatorname{ind}\left(E_{\left(\zeta^{\alpha}, 0\right)}^{\widetilde{\mathcal{X}}}\right)$ has largest cardinal $\lambda=\lambda\left(E_{\left(\zeta^{\alpha}, 0\right)}^{\widetilde{\mathcal{X}}}\right)$ and

$$
\lambda<\operatorname{ind}\left(F_{\alpha}\right)<\operatorname{ind}\left(E_{\left(\zeta^{\alpha}, 0\right)}^{\tilde{\mathcal{X}}}\right) .
$$

We have $\beta+1 \notin \mathscr{D}^{\overline{\mathcal{U}}}$, so $\left(\kappa^{+}\right)^{M_{\alpha}^{\bar{u}}} \leq \operatorname{ind}\left(\bar{E}_{\alpha}\right)$, so $\left(\mu^{+}\right)^{M_{\zeta^{\alpha}}^{\widetilde{\mathcal{X}}}} \leq \operatorname{ind}\left(F_{\alpha}\right)$, so by (8), $\mu<\lambda$. Since $\mu \in \operatorname{rg}\left(i_{\lambda^{\alpha} \zeta^{\alpha}}^{\tilde{\mathcal{X}}}\right)$, it follows that $\mu<\operatorname{cr}\left(E_{\left(\zeta^{\alpha}, 0\right)}^{\widetilde{\mathcal{X}}}\right)$, so $\xi \leq \varepsilon=$ $\operatorname{pred}^{\tilde{\mathcal{X}}}\left(\zeta^{\alpha}\right)$, as required. The rest is now clear.

From the preceding discussion, it follows that $\lambda^{\beta+1} \in b^{\mathcal{X}^{\beta+1}},\left(\lambda^{\beta+1}, \infty\right]_{\mathcal{X}^{\beta+1}}$ does not drop in model or degree and

$$
\operatorname{cr}\left(i_{\lambda^{\beta+1} \infty}^{\mathcal{X}^{\beta+1}}\right)=i_{\lambda^{\beta+1}}^{* \widetilde{\mathcal{X}}}\left(\operatorname{cr}\left(i_{\xi \infty}^{\mathcal{X}^{\alpha}}\right)\right)
$$

So the left diagram in Figure 11 is at least plausible.

It remains to verify that the diagram commutes. The diagram which results if we remove $\pi_{\beta+1}$ from Figure 11, is already known to commute, by induction and facts about $\Sigma^{\text {st }}$. We have

$$
\pi_{\beta+1} \circ i_{\bar{E}_{\beta}}^{M_{\alpha}}=i_{\lambda^{\beta+1}}^{* \widetilde{\mathcal{X}}} \circ i_{\lambda^{\alpha} \xi}^{\widetilde{\mathcal{X}}} \circ \pi_{\alpha}
$$

by properties of essential tree embeddings.

For each $\varepsilon$, write $\varrho_{\varepsilon}=\varrho_{\varepsilon} \circ \varphi_{\varepsilon}$. Let $j=i_{\lambda^{\beta+1} \infty}^{\mathcal{X}^{\beta+1}}$. It just remains to see that

$$
\varrho_{\beta+1}=\varrho_{\beta+1} \circ \varphi_{\beta+1}=j \circ \pi_{\beta+1} .
$$

For simplicity let us assume that $m=0$; for $m>0$ it is analogous.

Let $x=i_{\bar{E}_{\beta}}^{M_{\alpha}}(f)(a) \in M_{\beta+1}$, where $a \in\left[\nu\left(\bar{E}_{\beta}\right)\right]^{<\omega}$ and $f \in M_{\alpha}$. Then

$$
\varrho_{\beta+1}(x)=\psi_{\alpha, \beta+1}\left(\varrho_{\alpha}(f)\right)\left(\varrho_{\beta}(a)\right),
$$

since $\varrho_{\beta+1}=\varrho_{\beta+1}^{\prime} \circ \bar{\varrho}_{\beta+1}$ and

$$
\bar{\varrho}_{\beta+1}\left(\varphi_{\beta+1}(x)\right)=i_{F_{\beta}}^{M_{\infty}^{\mathcal{X}^{\alpha}}}\left(\varrho_{\alpha}(f)\right)\left(\varrho_{\beta}(a)\right),
$$

and as discussed earlier, $\operatorname{cr}\left(\varrho_{\beta+1}^{\prime}\right)>\lambda\left(E_{\zeta^{\beta}}^{\widetilde{\mathcal{X}}}\right)>\max \left(\varrho_{\beta}(a)\right)$.

On the other hand,

$$
j\left(\pi_{\beta+1}(x)\right)=j(g(c))=j(g)(c)
$$

where $g=i_{\lambda^{\beta}+1}^{* \widetilde{\mathcal{X}}} \circ i_{\lambda^{\alpha} \xi}^{\widetilde{\mathcal{X}}} \circ \pi_{\alpha}(f)$ and $c=i_{\lambda^{\beta} \zeta^{\beta}}^{\widetilde{\mathcal{X}}}\left(\pi_{\beta}(a)\right)=j(c)$, since $\max (c)<$ $\nu\left(F_{\beta}\right) \leq \operatorname{cr}(j)$.

So it suffices to show that $j(g)=\psi_{\beta, \alpha+1}\left(\varrho_{\alpha}(f)\right)$ and $c=\varrho_{\beta}(a)$, as then the objects in lines (9) and (10) are equal, as desired. But $j(g)=\psi_{\beta, \alpha+1}\left(\varrho_{\alpha}(f)\right)$ by the commutativity already known in the left diagram of Figure 11; and by its right diagram and since $\max (c)<\operatorname{ind}\left(F_{\beta}\right)<\operatorname{cr}\left(i_{\zeta_{*}^{\beta} \infty}^{\mathcal{X}^{\beta}}\right)$, we have

$$
\varrho_{\beta}(a)=i_{\lambda^{\beta} \infty}^{\mathcal{X}^{\beta}}\left(\pi_{\beta}(a)\right)=i_{\lambda^{\beta} \zeta_{*}^{\beta}}^{\mathcal{X}^{\beta}}\left(\pi_{\beta}(a)\right)=i_{\lambda^{\beta} \zeta^{\beta}}^{\widetilde{\mathcal{X}^{\beta}}}\left(\pi_{\beta}(a)\right)=c,
$$

completing the proof of the theorem. 


\subsection{Dodd-Jensen and $\Sigma^{\text {st }}$}

10.8 Definition. Lifting Dodd-Jensen (DJ) is defined just like the DJ property, but with the class of $n$-lifting embeddings replacing near $n$-embeddings (when at degree $n$ ). Likewise for lifting weak DJ.

10.9 Remark. Assuming DC, given a sufficiently iterable countable premouse $M$ and an enumeration $e$ of $M$ in ordertype $\omega$, we can construct a strategy $\Sigma$ for $M$ with lifting weak DJ, completely analogously to the construction of one with (standard) weak DJ. Clearly lifting (weak) DJ implies weak DJ, because every near $n$-embedding is $n$-lifting.

10.10 Theorem. Let $\Sigma, \Omega, M$ be as in Theorem 9.1, with $M$ a premouse, and $\Sigma$ an $(m, \Omega+1)$-strategy for $M$, and suppose that $\operatorname{card}(M)<\Omega$. If $\Sigma$ has lifting $D J$ then so does $\Sigma^{\text {st }}$. If $M$ is countable and $e$ is an enumeration of $M$ in ordertype $\omega$, then likewise for lifting weak DJ with respect to $e$.

Proof. Suppose $M$ is $\lambda$-indexed. We literally give the proof for lifting DJ, but for lifting weak DJ it is essentially the same. Let $\overrightarrow{\mathcal{T}}$ be according to $\Sigma^{\text {st }}$, with $N=M_{\infty}^{\overrightarrow{\mathcal{T}}}$ and $n=\operatorname{deg}^{\overrightarrow{\mathcal{T}}}(\infty)$, and let $Q, \pi$ be such that $(Q, m) \unlhd(N, n)$ and $\pi: M \rightarrow Q$ is $m$-lifting.

We may assume that $\operatorname{lh}(\overrightarrow{\mathcal{T}})<\Omega$ and each normal tree in $\overrightarrow{\mathcal{T}}$ has length $<\Omega$, because $\operatorname{card}(M)<\Omega$ and $\Omega$ is regular. Let $\overrightarrow{\mathcal{U}}$ be the corresponding optimal $m$-maximal stack on $M$ given by the proof of Lemma 9.8. Let $N^{\prime}=M_{\infty}^{\overrightarrow{\mathcal{U}}}$ and $n^{\prime}=\operatorname{deg}^{\overrightarrow{\mathcal{U}}}(\infty)$. Letting $Q^{\prime} \unlhd N^{\prime}$ be the resulting lift of $Q$ and $\sigma: Q \rightarrow Q^{\prime}$ the restricted copy map, note that $\left(Q^{\prime}, m\right) \unlhd\left(N^{\prime}, n^{\prime}\right)$ and $\pi^{\prime}=\sigma \circ \pi: M \rightarrow Q^{\prime}$ is $m$-lifting.

Now $\mathcal{X}=\mathcal{W}^{\Sigma}(\overrightarrow{\mathcal{U}})$ is via $\Sigma$, of length $<\Omega$, and we have the $n^{\prime}$-lifting

$$
\varsigma=\varsigma^{\Sigma}(\overrightarrow{\mathcal{T}}): M_{\infty}^{\overrightarrow{\mathcal{T}}} \rightarrow M_{\infty}^{\mathcal{X}}
$$

Let $Q^{\prime \prime}=\varsigma\left(Q^{\prime}\right)$ if $Q^{\prime} \triangleleft N^{\prime}$, and $Q^{\prime \prime}=M_{\infty}^{\mathcal{X}}$ otherwise. Let $\pi^{\prime \prime}=\varsigma \circ \pi^{\prime}$. Then we can apply lifting DJ for $\Sigma$ to $\mathcal{X}, Q^{\prime \prime}, \pi^{\prime \prime}$. Therefore $Q^{\prime \prime}=M_{\infty}^{\mathcal{X}}\left(\right.$ so $\left.Q^{\prime}=N^{\prime}\right)$ and $b^{\mathcal{X}}$ does not drop in model or degree, so $n^{\prime}=m$, and for each $\alpha \in \mathrm{OR}^{M}$, we have $i^{\mathcal{X}}(\alpha) \leq \pi^{\prime \prime}(\alpha)$. Therefore $b^{\overrightarrow{\mathcal{U}}}$ does not drop in model or degree, so $i^{\mathcal{X}}=\pi^{\prime \prime} \circ i^{\overrightarrow{\mathcal{U}}}$. Therefore $i^{\overrightarrow{\mathcal{U}}}(\alpha) \leq \pi^{\prime}(\alpha)$ for each $\alpha<\mathrm{OR}^{M}$. But then, similarly, $b^{\overrightarrow{\mathcal{T}}}$ also does not drop in model or degree, $i^{\overrightarrow{\mathcal{U}}}=\sigma \circ i^{\overrightarrow{\mathcal{T}}}$ and $i^{\overrightarrow{\mathcal{T}}}(\alpha) \leq \pi(\alpha)$ for each $\alpha$, so we are done.

If instead $M$ is MS-indexed then combine the preceding argument with that in the proof of Claim 11 of the proof of Theorem 7.3.

10.11 Remark. One would like to be able to prove a version of the preceding theorem for standard (weak) DJ. We can prove this in certain cases, but do not see how to in general. This is because (considering $\Gamma^{\text {st }}$, where $\Gamma$ is the u-strategy corresponding to $\Sigma$ ) the lifting map $\varsigma: M_{\infty}^{\overrightarrow{\mathcal{T}}} \rightarrow M_{\infty}^{\mathcal{X}}$ need not be a near $\mathrm{u}$ - $n$-embedding where $n=\mathrm{u}-\operatorname{deg}^{\overrightarrow{\mathcal{T}}}(\infty)$. However, if either (i) $m>0$, or 
(ii) $M$ is passive, or (iii) $M$ is MS-indexed type 1 or 3 , then we do get (weak)

DJ for $\Sigma^{\text {st }}$. This is due to the following easy consequence of condensation.

Let $M, N$ be $n$-sound and $\varrho: M \rightarrow N$ be $n$-lifting $\vec{p}_{n}$-preserving. Suppose that $\varrho$ is not an $n$-embedding, and either (i) $n>0$, (ii) $M$ is passive, or (iii) $M$ is MS-indexed type 1 or 3 . Then there is some $Q$ such that

$$
\text { either } Q \triangleleft N \text {, or } Q \triangleleft \operatorname{Ult}\left(N \mid \rho, F^{N \mid \rho}\right) \text { for some } \rho \text {, }
$$

and an $n$-embedding $\pi^{\prime}: M \rightarrow Q$.

For let $\rho=\sup \pi$ " $\rho_{n}^{M}$. We have $\rho<\rho_{n}^{N}$ because $\pi$ is not an $n$-embedding.

If $n=0$ and $M$ is passive then clearly $Q=N \| \rho$ and $\pi^{\prime}=\pi$ works (but note maybe $N \mid \rho$ is active).

If $n=0$ and $M$ is MS-indexed type 3 then note that $\rho$ is a limit of generators of $F^{N}$, and let $Q \triangleleft N$ or $Q \triangleleft \operatorname{Ult}\left(N \mid \rho, F^{N \mid \rho}\right)$ be such that $F^{N}\left\lceil\rho=F^{Q}\right.$, and $\pi^{\prime}=\pi$ (note that in this case, $\operatorname{dom}(\pi)=M^{\mathrm{sq}}$ ).

Suppose $n=0$ and $M$ is MS-indexed type 1 . Let $\mu=\operatorname{cr}\left(F^{M}\right)$ and $\kappa=$ $\operatorname{cr}\left(F^{N}\right)=\pi(\mu)$. Let

$$
Q=\operatorname{cHull}_{0}^{N}(\kappa \cup \operatorname{rg}(\pi))
$$

and $\sigma: Q \rightarrow N$ be the uncollapse and $\pi^{\prime}: M \rightarrow Q$ be such that $\sigma \circ \pi^{\prime}=\pi$. Then

$$
\sup \sigma " \mathrm{OR}^{Q}=\sup \pi " \mathrm{OR}^{M}
$$

and $Q$ is a type 1 premouse by standard arguments, and $\pi^{\prime}$ is $\mathrm{r} \Sigma_{1}$-elementary. We have $Q \in N$ and $\left(\kappa^{+}\right)^{Q}<\left(\kappa^{+}\right)^{N}$ and

$$
F^{Q} \uparrow\left(\kappa^{+}\right)^{Q}=F^{N} \uparrow\left(\kappa^{+}\right)^{Q} .
$$

So basically by [17, §4], either $Q \triangleleft N$ or letting $\alpha=\left(\kappa^{+}\right)^{N}, N \mid \alpha$ is active and $Q \triangleleft \operatorname{Ult}\left(N \mid \alpha, F^{N \mid \alpha}\right)$, so we are done.

Now suppose $n>0$. Let $Q=\operatorname{cHull}_{n}^{N}\left(\rho \cup \vec{p}_{n}^{N}\right)$ and $\sigma: Q \rightarrow N$ be the uncollapse. Note that $\operatorname{rg}(\pi) \subseteq \operatorname{rg}(\sigma)$ and let $\pi^{\prime}: M \rightarrow Q$ be such that $\sigma \circ \pi^{\prime}=\pi$. Note that $Q$ is $(n-1)$-sound and $\pi^{\prime}$ is a near $(n-1)$-embedding, Note that $\pi^{\prime}\left(p_{n}^{M}\right)$ is $n$-solid for $Q$ and

$$
Q=\operatorname{Hull}_{n}^{Q}\left(\rho \cup \pi^{\prime}\left(\vec{p}_{n}^{M}\right)\right),
$$

so $\pi^{\prime}\left(p_{n}^{M}\right)=p_{n}^{Q} \backslash \rho$, but also because $\pi$ is $n$-lifting, therefore $\rho_{n}^{Q}=\rho$. So $Q$ is $n$-sound. Also, $Q \in N$. By condensation, either $Q \triangleleft N$ or $N \mid \rho$ is active and $Q \triangleleft \operatorname{Ult}\left(N \mid \rho, F^{N \mid \rho}\right)$. Moreover, because $\pi$ is $n$-lifting and $\rho_{n}^{Q}=\rho$ and $\pi^{\prime}\left(\vec{p}_{n}^{M}\right)=\vec{p}_{n}^{Q}, \pi^{\prime}$ is in fact an $n$-embedding, which suffices.

We conclude this segment with some simple corollaries pertaining to generic absoluteness of iterability under choice, and also constructing strategies with weak DJ in choiceless contexts.

10.12 Corollary. Let $\Omega>\omega$ be regular. Let $\mathbb{P}$ be an $\Omega$-cc forcing and let $G$ be $V$-generic for $\mathbb{P}$. Let $M$ be a countable $n$-sound premouse. Then: 
- If $V \models \mathrm{DC}+$ " $M$ is $(n, \Omega, \Omega+1)$-iterable" then $V[G] \models$ " $M$ is $(n, \Omega, \Omega+1)^{*}$ iterable".

- If $V[G] \models \mathrm{DC}+$ " $M$ is $(n, \Omega, \Omega+1)^{*}$-iterable" then $V \models$ " $M$ is $(n, \Omega, \Omega+1)^{*}$ iterable".

Proof. Assume DC and suppose $M$ is $(n, \Omega, \Omega+1)^{*}$-iterable. Then there is an $(n, \Omega+1)$-strategy $\Sigma$ for $M$ with weak DJ (note that the construction of such a strategy only uses $(n, \Omega, \Omega+1)^{*}$-iterability, not $(n, \Omega, \Omega+1)$-iterability), which by 4.47 has strong hull condensation. Therefore by $7.3, V[G]$ has an $(n, \Omega+1)$ strategy $\Sigma^{\prime}$ for $M$ with strong hull condensation. So by Theorem 9.1, $M$ is $(n, \Omega, \Omega+1)^{*}$-iterable in $V[G]$.

Now suppose instead that $V[G] \models \mathrm{DC}+$ " $M$ is $(n, \Omega, \Omega+1)^{*}$-iterable". Then in $V[G]$ there is an $(n, \Omega+1)$-strategy $\Sigma^{\prime}$ with weak DJ with respect to some enumeration $e \in V$ of $M$. By 7.6, $\Sigma=\Sigma^{\prime} \uparrow V \in V$ and $\Sigma$ has weak DJ in $V$. So by $9.1, \Sigma$ extends to an $(n, \Omega, \Omega+1)^{*}$-strategy in $V$.

Note that in the following corollary, $M$ is a premouse, not a wcpm.

10.13 Corollary. Assume ZFC. Let $M$ be a countable $m$-sound premouse and $e$ be an enumeration of $M$ in ordertype $\omega$. Let $m<\omega$. Let $\Omega>\omega$ be regular. Let $\mathbb{P}$ be an $\Omega$-cc forcing and $G$ be $V$-generic for $\mathbb{P}$. Then the following are equivalent:

- There is an $(m, \Omega+1)$-strategy for $M$ with strong hull condensation.

- $M$ is $(m, \Omega, \Omega+1)^{*}$-iterable.

- There is an $(m, \Omega+1)$-strategy for $M$ with weak $D J$ with respect to $e$.

- $V[G]$ satisfies one of the preceding statements.

Proof of Corollary 10.13. Both $V$ and $V[G]$ satisfy ZFC, so the previous corollary and its arguments apply (note that $e \in V$ ), which easily yields 10.13.

\subsection{Weak DJ without DC}

We now discuss some choiceless constructions of strategies with weak DJ. The main result is Corollary 10.17, and the basic idea for that may have originated from some observations of Dominik Adolf (that is, using Theorem 7.3 to extend a strategy in $\mathrm{HOD}_{X}$ via Vopenka forcing). However, we start with Corollary 10.14 below, which is actually less general, but the two proofs are different, and both seem of interest.

10.14 Corollary. Let $\Omega>\omega$ be regular and suppose that for no $\alpha<\Omega$ is $\Omega$ the surjective image of $V_{\alpha}$. Let $M$ be a countable $m$-sound premouse. Let $\Sigma$ be an $(m, \Omega+1)$-strategy for $M$ with strong hull condensation. Let $e$ be an enumeration of $M$ in ordertype $\omega$. Then there is an $(m, \Omega+1)$-strategy for $M$ with weak DJ with respect to $e$. 
10.15 Remark. Before proving the corollary, we sketch another proof scenario, which other than the extension of $\Sigma$ to the stacks strategy $\Sigma^{\text {st }}$, would only use standard techniques if it could be made to work, and point out where the scenario seems to run into problems. First extend $\Sigma$ to $\Sigma^{\text {st }}$, and then attempt a choiceless variant of the construction of a strategy with weak DJ from $\Sigma^{\text {st }}$. (Note that we are not assuming DC, which the usual construction uses.) A natural attempt for the latter is as follows.

Let $\alpha_{0}$ be the least $\alpha$ such that there is $\overrightarrow{\mathcal{T}} \in V_{\alpha}$ with $\overrightarrow{\mathcal{T}}$ according to $\Sigma^{\text {st }}$, and some $Q \unlhd M_{\infty}^{\overrightarrow{\mathcal{T}}}$ and $\pi: M \rightarrow Q$ violating weak DJ. Let $A_{0}$ be the set of all such pairs $(\overrightarrow{\mathcal{T}}, Q)$ where $\overrightarrow{\mathcal{T}} \in V_{\alpha_{0}}$. For $(\overrightarrow{\mathcal{T}}, Q) \in A_{0}$, let $\Sigma_{\overrightarrow{\mathcal{T}}, Q}^{\text {st }}$ by the strategy for $Q$ given by the tail of $\Sigma^{\text {st }}$.

Now for each such $(\overrightarrow{\mathcal{T}}, Q) \in A_{0}$, define a tree $\mathcal{U}_{\overrightarrow{\mathcal{T}}, Q}$ on $Q$, via $\Sigma_{\overrightarrow{\mathcal{T}}, Q}^{\text {st }}$, with these trees resulting from the simultaneous comparison of the $Q$ 's (but note that for a given $Q$, there could be multiple corresponding trees $\overrightarrow{\mathcal{T}}$, and so multiple corresponding $\mathcal{U}_{\overrightarrow{\mathcal{T}}, Q}$ 's). This comparison terminates in $<\Omega$ stages, because if we reached stage $\Omega+1$, then working in $L\left(X, V_{\alpha_{0}}\right)$, where $X$ is a subset of $V_{\alpha_{0}} \times \mathrm{OR}$ coding the comparison, including final branches, we can form a hull of $V$ and reach the usual contradiction. Now for some $(\overrightarrow{\mathcal{T}}, Q) \in A_{0}, b^{\mathcal{U}_{\overrightarrow{\mathcal{T}}, Q}}$ does not drop in model or degree. Choosing such a $(\overrightarrow{\mathcal{T}}, Q)$ with $\operatorname{OR}\left(M_{\infty}^{\mathcal{U}_{\vec{\tau}, Q}}\right)$ least possible, let $Q^{\prime}=M_{\infty}^{\mathcal{U}_{\vec{\tau}, Q}}$. Then there is some $\pi^{\prime}: M \rightarrow Q^{\prime}$ witnessing a failure of weak DJ, and note that we have defined $Q^{\prime}$ outright from $\Sigma^{\text {st }}$. We can also set $\pi^{\prime}$ to be the $e$-lexicographically least witness.

However, there could be multiple pairs $(\overrightarrow{\mathcal{T}}, Q)$ with $Q^{\prime}=M_{\infty}^{\mathcal{U}_{\vec{\tau}, Q}}$. Thus, we don't seem to have a uniquely specified tail of $\Sigma^{\text {st }}$ for iterating $Q^{\prime}$. We do have only $V_{\alpha_{0}}$-many such pairs, so only $V_{\alpha_{0}}$-many strategies for $Q^{\prime}$. So we might continue by looking for failures of weak DJ arising from each of these strategies, comparing these and so on. But after repeating this process $\omega$-many times, we seem to need DC in order to choose some bad stack via some specific strategy, in order to reach a contradiction. Thus, we do not see how to complete the proof in this scenario.

We now give a proof that does work. We first need a forcing lemma.

10.16 Lemma. Let $\Omega>\omega$ be regular and suppose that for no $\alpha<\Omega$ is $\Omega$ the surjective image of $V_{\alpha}$. Then there is a homogeneous $\Omega$-cc forcing $\mathbb{P}$ which forces $\mathrm{CH}$, in the strong sense that $\Omega=\omega_{1}^{V^{\mathbb{P}}}=\left(2^{\aleph_{0}}\right)^{V^{\mathbb{P}}}=\operatorname{card}^{V^{\mathbb{P}}}\left(\mathrm{HC}^{V^{\mathbb{P}}}\right)$.

Proof. Let $\mathbb{P}$ be the forcing whose conditions are functions $p$ with $\operatorname{dom}(p)$ a finite set $\subseteq(0, \Omega) \times \omega$ and $p(\alpha, n) \in V_{\alpha}$ for each $(\alpha, n) \in \operatorname{dom}(p)$, and with ordering $p \leq q$ iff $q \subseteq p$. We claim that $\mathbb{P}$ works.

For clearly $\mathbb{P}$ is homogeneous. Let $G$ be $V$-generic and $g=\bigcup G$. Clearly and

$$
g:(0, \Omega) \times \omega \rightarrow V_{\Omega}
$$

is a surjection; in fact, for each $\alpha \in(0, \Omega)$, the function $n \mapsto g(\alpha, n)$ is a surjection $\omega \rightarrow V_{\alpha}$. So $\Omega \leq \omega_{1}^{V[G]}$ and it suffices to see that $\mathbb{P}$ is $\Omega$-cc and for 
each $x \in \mathrm{HC}^{V[G]}$ there is a $\mathbb{P}$-name $\dot{x} \in V_{\Omega}$ such that $\dot{x}^{G}=x$.

Claim 1. $\mathbb{P}$ is $\Omega$-cc.

Proof. Let $\lambda \in \mathrm{OR}$ and $\left\langle A_{\alpha}\right\rangle_{\alpha<\lambda}$ be a $\lambda$-pre-antichain of $\mathbb{P}$. We must see that $\lambda<\Omega$. So suppose $\lambda=\Omega$. The proof is just a choiceless variant of the usual $\Delta$-system argument.

For each $p \in \mathbb{P}, \operatorname{dom}(p)$ is just a finite set of pairs of ordinals. So by reducing each $A_{\alpha}$ if necessary, may assume that we have $\left\langle d_{\alpha}\right\rangle_{\alpha<\Omega}$ such that $\operatorname{dom}(p)=d_{\alpha}$ for all $p \in A_{\alpha}$, for all $\alpha$. In $L\left[\left\langle d_{\alpha}\right\rangle_{\alpha<\Omega}\right]$, where we have ZFC (and $\Omega$ is regular) we can use the $\Delta$-system lemma. So we may assume that we have some fixed finite $d \subseteq \Omega \times \omega$ such that $d_{\alpha} \cap d_{\beta}=d$ for all $\alpha<\beta<\Omega$. Let $\gamma<\Omega$ be such that $d \subseteq \gamma \times \omega$. Then for each $\alpha$ and $p \in A_{\alpha}$, we have $p\left\lceil d \in V_{\gamma+\omega}\right.$.

Let $X=\left\{p|d| p \in A_{\alpha}\right.$ and $\left.\alpha<\Omega\right\}$. So $X \subseteq V_{\gamma+\omega}$. For $x \in X$, let $\alpha_{x}$ be the least $\alpha$ such that $x=p\left\lceil d\right.$ for some $p \in A_{\alpha}$. Then since there is no surjection $V_{\gamma+\omega} \rightarrow \Omega$ and $\Omega$ is regular, we may fix $\beta>\sup _{x \in X} \alpha_{x}$. Let $q \in A_{\beta}$. Let $x=q\left\lceil d\right.$. Then $x \in X$. Let $\alpha=\alpha_{x}$. Then $\alpha<\beta$. Let $p \in A_{\alpha}$ be such that $x=p\lceil d$. Then we have $p\lceil d=x=q\lceil d$, but since $d=\operatorname{dom}(p) \cap \operatorname{dom}(q)$, it follows that $p, q$ are compatible, a contradiction.

Claim 2. For each $x \in \mathcal{P}(\omega)^{V[G]}$ there is a $\mathbb{P}$-name $\dot{x} \in V_{\Omega}$ such that $\dot{x}^{G}=x$.

Proof. Let $\tau$ be a $\mathbb{P}$-name for $x$. For $n<\omega$, let $B_{n}=\{p \in \mathbb{P} \mid p \| \check{n} \in \tau\}$. Let $\left\langle d_{\alpha}\right\rangle_{\alpha<\Omega}$ enumerate $[\Omega \times \omega]^{<\omega}$. For $p \in \mathbb{P}$, let $\alpha_{p}$ be the $\alpha$ such that $\operatorname{dom}(p)=d_{\alpha}$. Define a set $C_{n} \subseteq B_{n}$, determining whether $p \in C_{n}$ recursively on $\alpha_{p}$, as follows: given $p \in B_{n}$, put $p \in C_{n}$ iff $p \perp q$ for all $q \in C_{n}$ such that $\alpha_{q}<\alpha_{p}$. Note that $C_{n} \in V_{\Omega}$, as otherwise we easily get an $\Omega$-pre-antichain. And $C_{n}$ is pre-dense in $B_{n}$, because if $p \in B_{n} \backslash C_{n}$ then $p \| q$ for some $q \in C_{n}$ with $\alpha_{q}<\alpha_{p}$. So let $\dot{x}$ be the $\mathbb{P}$-name consisting of all pairs $(p, \check{n})$ such that $n<\omega$ and $p \in C_{n}$. It follows that $\dot{x} \in V_{\Omega}$ and $\dot{x}_{G}=x$, as desired.

This completes the proof of the lemma.

Proof of Corollary 10.14. Let $\mathbb{P}$ be the forcing of 10.16 and $G$ be $V$-generic for $\mathbb{P}$. So $\mathbb{P}$ is homogeneous, $\Omega$-cc and $V[G]$ has a bijection $f: \Omega=\aleph_{1}^{V[G]} \rightarrow \mathrm{HC}^{V[G]}$. Let $\Sigma^{\prime}$ be the extension of $\Sigma$ to $V[G]$ given by 7.3 .

Work in $V[G]$. So $\Sigma^{\prime}$ is an $\left(m, \omega_{1}+1\right)$-strategy with strong hull condensation, and $\omega_{1}$ is regular. Using $\left(\Sigma^{\prime}\right)^{\text {st }}$ and the bijection $f$, we can run the usual construction of an $\left(m, \omega_{1}+1\right)$-strategy $\Lambda^{\prime}$ for $M$ with weak DJ with respect to $e$. As mentioned in $4.46, \Lambda^{\prime}$ is the unique such strategy for $M$.

But then $\Lambda==_{\operatorname{def}} \Lambda^{\prime} \uparrow V \in V$ (because $\mathbb{P}$ is homogeneous and $\Lambda^{\prime}$ is unique; alternatively, use 7.6), and $\Lambda$ has weak DJ with respect to $e$ in $V$.

We now slightly improve on 10.14. But this time, the proof works by executing the AC part of the argument in an inner model of choice, instead of a forcing extension. As mentioned above, the idea of Using Theorem 7.3 to extend a strategy of $\operatorname{HOD}_{X}$ via Vopenka may have come from observations of Dominik Adolf. 
10.17 Corollary. Let $\Omega>\omega$ be regular and such that for no $\alpha<\Omega$ is $\Omega$ the surjective image of $\mathcal{P}(\alpha)$. Let $M$ be a countable $m$-sound $(m, \Omega, \Omega+1)^{*}$ iterable premouse and $e$ be an enumeration of $M$ in ordertype $\omega$. Then there is an $(m, \Omega+1)$-iteration strategy for $M$ with weak $D J$ with respect to $e$.

Proof. Note that $\Omega$ is inaccessible in every proper class inner model $H$ of ZFC. When we mention weak DJ below, we mean with respect to $e$.

Let $\Sigma$ be an $(m, \Omega, \Omega+1)^{*}$-strategy for $M$. Let $H=\operatorname{HOD}_{\Sigma, M, e}$ and $\Lambda=\Sigma \uparrow$ $H$. So $\Lambda, M, e \in H$ and

$$
H \models \mathrm{ZFC}+\text { " } \Omega \text { is inaccessible and } \Lambda \text { is an }(m, \Omega, \Omega+1)^{*} \text {-strategy for } M \text { ". }
$$

So there is (a unique) $\Psi \in H$ such that

$$
H \models \text { " } \Psi \text { is an }(m, \Omega+1) \text {-strategy for } M \text { with weak DJ". }
$$

For each $\alpha<\Omega$ and $X \subseteq \alpha$, let $G_{X}$ be the Vopenka generic for adding $X$ to $H$. This Vopenka forcing has the $\Omega$-cc in $H$, because $\Omega$ is not the surjective image of $\mathcal{P}(\alpha)$ in $V$. Also, $H\left[G_{X}\right]=\operatorname{HOD}_{\Sigma, M, e, X}$. Given $\beta<\Omega$ and $Y \subseteq \beta$, let $G_{Y}^{X}$ be the Vopenka generic for adding $Y$ to $H\left[G_{X}\right]$. This forcing is $\Omega$-cc in $H\left[G_{X}\right]$.

So by 7.3 , there is a unique $\Psi_{X} \in H\left[G_{X}\right]$ such that

$$
H\left[G_{X}\right] \models \text { " } \Psi_{X} \text { is an }(m, \Omega+1) \text {-strategy with weak DJ"; }
$$

moreover, $\Psi \subseteq \Psi_{X}$. Similarly, there is a unique $\Psi_{Y}^{X}$ for $H\left[G_{X}\right]\left[G_{Y}^{X}\right]$, and $\Psi_{X} \subseteq$ $\Psi_{Y}^{X}$. Note that

$$
H\left[G_{X}\right]\left[G_{Y}^{X}\right]=\operatorname{HOD}_{\Sigma, M, e, X, Y}=H\left[G^{Y}\right]\left[G_{X}^{Y}\right],
$$

so $\Psi_{Y}^{X}=\Psi_{X}^{Y}$, so $\Psi_{X}$ is compatible with $\Psi_{Y}$.

Let $\Psi_{X}^{\Omega}$ be the restriction of $\Psi_{X}$ to an $(m, \Omega)$-strategy of $H\left[G_{X}\right]$, and let $\Psi^{\Omega}$ be the union of all $\Psi_{X}^{\Omega}$ (over all bounded subsets $X$ of $\Omega$ ).

Then clearly $\Psi^{\Omega}$ is an $(m, \Omega)$-strategy with weak DJ. In fact, $\Psi^{\Omega}$ is the unique such strategy, because otherwise we can run the usual phalanx comparison argument working inside some inner model of ZFC, using the fact that $\Omega$ is inaccessible there, to see that the comparison terminates.

We claim that $\Psi^{\Omega}$ extends (uniquely) to an $(m, \Omega+1)$-strategy. For given any tree $\mathcal{T}$ via $\Psi^{\Omega}$ of length $\Omega$, we can argue as above with $H_{\mathcal{T}}=\operatorname{HOD}_{\Sigma, M, e, \mathcal{T}}$ replacing $H$. Let $\Psi_{\mathcal{T}} \in H_{\mathcal{T}}$ be the resulting $(m, \Omega+1)$-strategy of $H_{\mathcal{T}}$, and $\Psi_{\mathcal{T}}^{\Omega} \in V$ the resulting $(m, \Omega)$-strategy of $V$. Then $\Psi^{\Omega}=\Psi_{\mathcal{T}}^{\Omega}$ by the uniqueness mentioned of $\Psi^{\Omega}$. But $\Psi_{\mathcal{T}}$ is compatible with $\Psi_{\mathcal{T}}^{\Omega}=\Psi^{\Omega}$, so $\mathcal{T}$ is via $\Psi_{\mathcal{T}}$, and since $\mathcal{T} \in H \models$ " $\Psi_{\mathcal{T}}$ is an $(m, \Omega+1)$-strategy", therefore $\Psi_{\mathcal{T}}(\mathcal{T})$ is a $\mathcal{T}$-cofinal branch, as desired.

So let $\Psi^{+}$be this extension of $\Psi^{\Omega}$. Then $\Psi^{+}$has weak DJ, completing the proof. For if we have some counterexample to weak DJ given by a tree $\mathcal{T}$ of length $\Omega+1$, note that by the regularity of $\Omega$, there is some $\alpha \in b^{\mathcal{T}}$ such that $\Psi \uparrow(\alpha+1)$ is also a counterexample, contradicting weak DJ for $\Psi^{\Omega}$. 


\section{Index}

$\left(I_{\xi}^{\alpha}\right)^{\mathcal{T} \rightsquigarrow \mathcal{X}},\left(\pi_{\xi}^{\alpha}\right)^{\mathcal{T} \rightsquigarrow \mathcal{X}}$, etc, 71

$(\mathcal{T}, b), \mathcal{T}^{\curlywedge} b, 22$

$(\alpha, \beta]_{\mathcal{T}}$ etc, 10

(u- $k, \theta$ )-iterability, 16

*-tree embedding, 31

$<^{\mathcal{T}}, 9$

$<^{\mathcal{X} / \mathcal{T}}, 72$

A-bad, 47

$E$-inflation, 35

$E_{\gamma}^{\Pi}, 31$

$E_{\alpha}^{\mathcal{T} \rightsquigarrow \mathcal{X}}, 38$

$F^{M}, 8$

$I_{\beta}, 24,28$

$L^{\alpha}, 71$

$M|\alpha, M| \mid \alpha, 9$

$M_{\infty}^{\mathcal{T}}, i_{0 \infty}^{\mathcal{T}}$, etc, 10

$M^{\mathrm{pv}}, 8$

$M^{\text {sq }}, M^{\text {unsq }}, 9$

$M_{\beta}^{* \mathcal{T}}, 10$

$M_{\infty}^{\overrightarrow{\mathcal{T}}}, 11$

$P_{\beta}, 24,28$

$Q_{\beta}, 27,28$

$Q_{\beta i}, 29$

$\mathbb{B}_{\delta}, 47$

$\mathcal{G}(M, \ldots)^{*}, 11,12$

$\mathcal{G}_{\text {fin }}, 3,78$

$\mathcal{G}_{\text {opt }}, 12$

$\operatorname{ind}(E), 9,20$

$\mathcal{L}_{\mathrm{LST}}, \mathcal{L}_{\mathrm{LST}}^{+}, 20$

$M_{\beta \kappa}, \gamma_{\beta \kappa}, \pi_{\beta \kappa}$, etc, 29

$\Pi:(\mathcal{T}, \theta) \hookrightarrow \mathcal{X}, 24,28$

$\Pi: \mathcal{T} \hookrightarrow \mathcal{X}, 29$

$\Pi: \mathcal{T} \hookrightarrow^{*} \mathcal{X}, 31$

$\Pi: \mathcal{T} \hookrightarrow$ alm $\mathcal{X}, 35$

$\Pi^{\alpha \beta}$ (tree embedding), 74

$\Sigma^{\mathrm{nm}}, 100$

$\Sigma^{\mathrm{st}}, 77,97$

$\mathcal{T}$-copying, 36

$\mathcal{T}$-inflationary, 35,36

$\mathcal{T}$-normal, 22

$\mathcal{T}$-special, 17

$\mathcal{T}$-very special, $\mathcal{T}$-vs, 17
$\mathcal{T}^{\wedge}\langle E\rangle, 22$

$\mathcal{T} \rightsquigarrow \mathcal{X}, 38,71$

$\mathcal{T} \geq \alpha, 72$

Ult, 8

$\mathrm{Ult}_{\mathrm{u}-n}, 14$

$\Upsilon_{\mathcal{T}}^{\Sigma}, 83,92$

$\mathcal{W}^{\Sigma}, 77,97$

$\mathcal{W}_{\mathcal{T}}^{\Sigma}, 84,92$

$\mathcal{X}^{\alpha}, t^{\alpha}$, etc, 71

clint, 23

complete $^{\Sigma}(\mathcal{T}), 41$

$\operatorname{cr}(E), 8$

dds, ddd, 23

$\operatorname{deg}^{\mathcal{T}}, 10$

$\operatorname{deg}^{\overrightarrow{\mathcal{T}}}(\infty), 11$

$\delta(\mathcal{T}), 10$

$\delta_{\beta}, 24$

$\mathscr{D}^{\mathcal{T}}, 9$

$\mathscr{D}_{\mathrm{deg}}^{\mathcal{T}}, 10$

$\mathbb{E}^{M}, \mathbb{E}_{+}^{M}, 8,22$

$\eta_{\delta}, 71$

$\mathrm{ex}_{\alpha}^{\mathcal{T}}, 10$

$\gamma_{\beta}, 24$

$\gamma_{\Pi \beta}, I_{\Pi \beta}$, etc, 29

$\unlhd, \triangleleft, 8,9$

$\iota^{M}, \iota_{\alpha}^{\mathcal{T}}, 22$

$\lambda$-indexed, 8

$\lambda(E), 8$

$\lambda^{\alpha \beta}, \kappa^{\alpha \beta}$, etc (inflation), 74

$\operatorname{lgcd}(M), 9$

$\operatorname{lh}(E), 8$

$\operatorname{lh}(\mathcal{T}), 9$

$\operatorname{lh}(\mathcal{T})^{-}, 10$

$\mathscr{T}$-strategy, 39

$\varrho_{\alpha}, 84$

$\nu(E), 8$

$\widetilde{\nu}^{M}, 13$

$\widetilde{\nu}_{\alpha}^{\mathcal{T}}, \widetilde{\nu}\left(E_{\alpha}^{\mathcal{T}}\right), 11$

$\omega$-mice, 78

$\omega_{\theta}^{\alpha \beta}, 76$

$\omega_{\beta}, 27,28$

$\pi_{\theta}^{\alpha \beta}, \pi_{\theta \kappa}^{\alpha \beta}, 76$ 
$\pi_{\beta}, 24,28$

$\pi_{\infty}^{\mathcal{T} \rightsquigarrow \mathcal{X}}, 45$

$\operatorname{pred}^{\mathcal{T}}, 10$

$\psi_{\alpha \beta}, 86$

$\vec{p}_{n}$-preserving, 12

$\sigma_{\beta}, 28$

$\sigma_{x}, \sigma_{\alpha i}, 27$

$\varsigma^{\Sigma}, 97$

succ $^{\mathcal{T}}, 10$

$\tau_{\beta}, 28$

$\tau_{x}, \tau_{\alpha i}, 27$

$\theta^{\alpha}, 71$

u-n-maximal, 15

u- fine structure, 12

u-degree, 28

u-deg, 15

$\lfloor M\rfloor, 8$

$\mathrm{u} \rho_{n}, 13$

$\varrho(E), 8$

$\operatorname{wcof}_{k+1}^{M}, 83$

$\zeta^{\alpha}, 71$

$b^{\mathcal{T}}, 10$

$f^{\mathcal{T} \rightsquigarrow \mathcal{X}}, C^{\mathcal{T} \rightsquigarrow \mathcal{X}}$, etc, 38

$i_{E}^{M}, i_{E}^{M, m}, i_{E}, 8$

$i_{\alpha \beta}^{\mathcal{T}}, 10$

$i^{* \mathcal{T}}, 10$

$i_{\beta}^{* \mathcal{T}}, 10$

$i_{E}^{M, \mathrm{u}-n}, 14$

$i_{\beta}, 29$

$j_{\xi \eta}^{\mathcal{X}}, 29$

m-maximal, 10, 11

$n$-lifting, 12

$<^{(\alpha)}, 72$

$\lambda^{\alpha}, 71$

$\varrho_{\mathcal{T}}^{\Sigma}, 92$

$\Pi: \mathcal{T} \hookrightarrow_{\text {ess }} \mathcal{X}, 102$

$\Sigma^{\text {st }}, 97$

almost tree embedding, 35

artificial (drop), 11

bad, 47

bounding, 31

c-preserving, 12 chain condition, cc, 60

clint, 23

Closeness for $\mathrm{u}, 16$

coarse 0 -embedding, 20

commutativity of inflation, 52, 54

complete, 41

composition (tree embeddings), 59

convenient, 40, 41

copying, 36

correspondence of strategies, 18

correspondence of trees, 18

DJ, 43

dropdown, 23

easy, 84

essential, 101, 102

extender algebra, 47

extra inflationary, 41

factor tree, 72

genericity inflation, 47, 51

genericity iteration, 50

good (inflation), 71

inconvenient, 40, 41

inflation, 35, 36

inflation condensation, 39, 40

inflationary, 35, 36, 39, 40

ISC, 8

iterability (wcpm), 21

iteration class, 39

lifting, 12

lifting (weak) DJ, 110

maximal, 11

maximal ( $m$-maximal), 10

minimal simultaneous inflation, 46

MS-indexed, 8

near u-n-embedding, 13

nice, 12

normal, 22

normal pullback consistency, 101

normal tree (on wcpm), 21 
normal tree (wcpm), 21

one-step copy extension, 34

optimal (stack), 11

partial strategies, 99

partial strategy, 39

pending, 45

plus-strong hull condensation, 102

pre-antichain, 60

pre-inflationary, 39

premouse, 8

preserving, 12

pullback consistency, 101

putative, 16, 21, 39

putative stack, 11

putative tree, 11

regularly $\Xi$-total, 40

reverse, 23

segmented-premouse, seg-pm, 9

self iterability, 49

slight coherence, 20

special, 17

stack, 11

stack (on wcpm), 21

strong closeness, 32

strong hull condensation, 41

suitable (extender), 8

superstrong type, 8

terminal, 45

terminally non-dropping, 45

terminally-(non)-(model)-dropping, 45

translatability, 33

tree embedding, 24, 28, 29, 31

trivial (tree embedding), 31

type, 36

type A, B, 13

$\mathrm{u} \rho_{n}, 13$

u-deg, 15

u- $n$-embedding, 13

$\mathrm{u}$ - $n$-lifting (embedding), 13

u-n-maximal, 15

u-n-sound, 13 u-fine structure, 12

ultrapower, 8

uniqueness, tree embeddings, 31

universe (of a structure), 8

unravelled, 17

unravelling, 17, 71

very special, vs, 17

wcof, 83

wcpm, 19

weak coarse premouse, 19

weak cofinality, 83 


\section{References}

[1] E. Closson. The Solovay Sequence in Derived Models Associated to Mice. $\mathrm{PhD}$ thesis, University of California, Berkeley, 2008.

[2] Ronald Jensen. Manuscript on fine structure, inner model theory, and the core model below one Woodin cardinal. Available at http://www-irm.mathematik.hu-berlin.de/ raesch/org/jensen.html.

[3] Ronald Jensen. Smooth iterations. Available at http://www-irm.mathematik.hu-berlin.de/ raesch/org/jensen.html.

[4] William J. Mitchell and John R. Steel. Fine structure and iteration trees, volume 3 of Lecture Notes in Logic. Springer-Verlag, Berlin, 1994.

[5] Itay Neeman and John R. Steel. A weak Dodd-Jensen lemma. The Journal of Symbolic Logic, 64(3):1285-1294, 1999.

[6] Grigor Sargsyan. Hod mice and the mouse set conjecture, volume 236(1111) of Memoirs of the AMS. American Mathematical Society, 2015.

[7] Grigor Sargsyan, Ralf Schindler, and Farmer Schlutzenberg. Varsovian models II. In preparation.

[8] E. Schimmerling. Combinatorial principles in the core model for one woodin cardinal. Annals of Pure and Applied Logic, 74, 1995.

[9] Ernest Schimmerling and John R. Steel. Fine structure for tame inner models. The Journal of Symbolic Logic, 61(2):621-639, 1996.

[10] Ralf Schindler and John R. Steel. The self-iterability of $L[E]$. The Journal of Symbolic Logic, 74(3):751-779, 2009.

[11] Farmer Schlutzenberg. Background construction for $\lambda$-indexed mice. arXiv:2101.00889.

[12] Farmer Schlutzenberg. The definability of $\mathbb{E}$ in self-iterable mice. arXiv: 1412.0085 .

[13] Farmer Schlutzenberg. The definability of the extender sequence $\mathbb{E}$ from $\mathbb{E}\left\lceil\aleph_{1}\right.$ in $L[\mathbb{E}]$. arXiv: 1906.00276 .

[14] Farmer Schlutzenberg. Fine structure from normal iterability. arXiv:2011.10037.

[15] Farmer Schlutzenberg. Notes on $*$-translation. Available upon request.

[16] Farmer Schlutzenberg. Ordinal definability in $L[\mathbb{E}]$. arXiv:2012.07185.

[17] Farmer Schlutzenberg. Measures in mice. PhD thesis, UC Berkeley, 2007. arXiv:1301.4702. 
[18] Farmer Schlutzenberg. A premouse inheriting strong cardinals from $V$. Annals of Pure and Applied Logic, 171(9), 2020.

[19] Farmer Schlutzenberg and John R. Steel. A stationary tower free scale construction. In preparation.

[20] Farmer Schlutzenberg and Nam Trang. The fine structure of operator mice. arXiv:1604.00083.

[21] John R. Steel. Normalizing iteration trees and comparing iteration strategies. Forthcoming book available at http://math. berkeley. edu/ steel.

[22] John R. Steel. The core model iterability problem. Number 8 in Lecture Notes in Logic. Springer-Verlag, 1996.

[23] John R. Steel. Core models with more Woodin cardinals. The Journal of Symbolic Logic, 67(3):1197-1226, 2002.

[24] John R Steel. An outline of inner model theory. In Matthew Foreman and Akihiro Kanamori, editors, Handbook of set theory, pages 1595-1684. Springer, 2010. DOI:10.1007/978-1-4020-5764-9.

[25] Martin Zeman. Inner models and large cardinals, volume 5 of de Gruyter Series in Logic and its Applications. Walter de Gruyter \& Co., Berlin, 2002. 